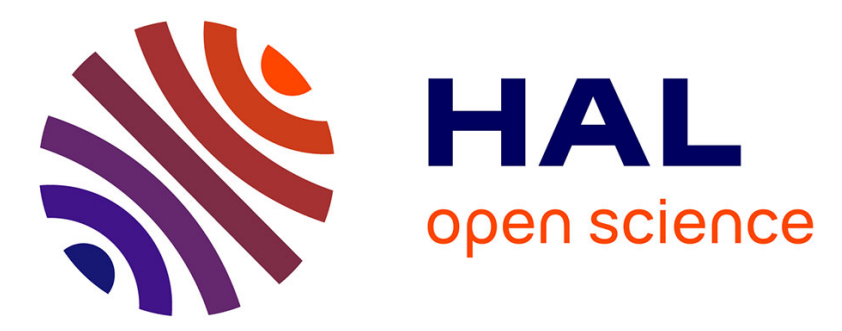

\title{
Le carnyx et le casque-oiseau celtiques de Tintignac (Naves-Corrèze). Description et étude technologique.
}

Christophe Maniquet, Thierry Lejars, Barbara Ambruster, Michel Pernot, Monique Drieux-Daguerre, Pascal Mora, Loïc Espinasse

\section{To cite this version:}

Christophe Maniquet, Thierry Lejars, Barbara Ambruster, Michel Pernot, Monique Drieux-Daguerre, et al.. Le carnyx et le casque-oiseau celtiques de Tintignac (Naves-Corrèze). Description et étude technologique.: Dossier "Tintignac". Aquitania, 2012, Aquitania, 27, pp.63-150. hal-01922481

\section{HAL Id: hal-01922481 \\ https://hal.science/hal-01922481}

Submitted on 14 Nov 2018

HAL is a multi-disciplinary open access archive for the deposit and dissemination of scientific research documents, whether they are published or not. The documents may come from teaching and research institutions in France or abroad, or from public or private research centers.
L'archive ouverte pluridisciplinaire HAL, est destinée au dépôt et à la diffusion de documents scientifiques de niveau recherche, publiés ou non, émanant des établissements d'enseignement et de recherche français ou étrangers, des laboratoires publics ou privés. 


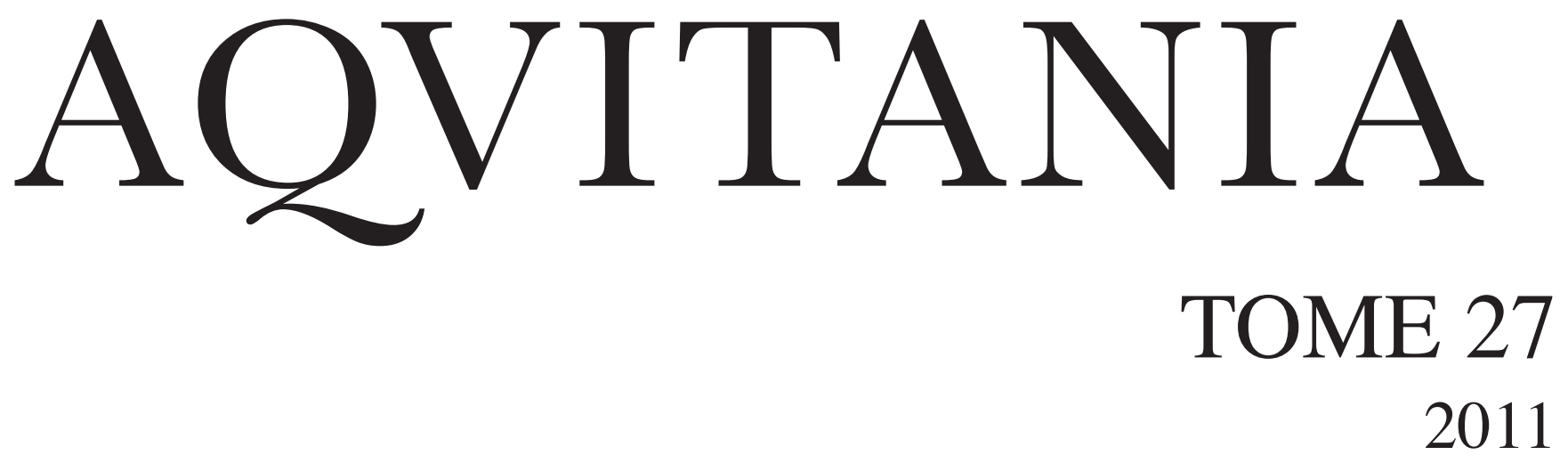

Revue interrégionale d'archéologie

\author{
Aquitaine \\ Limousin \\ Midi-Pyrénées \\ Poitou-Charentes
}

Revue publiée par la Fédération Aquitania, avec le concours financier

du Ministère de la Culture, Direction du Patrimoine, Sous-Direction de l'Archéologie

et de l'Université Michel de Montaigne - Bordeaux,

et soutenue par l'Institut des Sciences Humaines et Sociales du CNRS 


\section{SOMMAIRE}

Auteurs

A. Dumas, A. Dautant, Th. Constantin, A. Beschi

La sépulture du premier âge du Fer de Cablanc (Barbaste, Lot-et-Garonne)

TH. LE DREFF

Fours et ateliers de potiers au second âge du Fer dans l'isthme gaulois.

$19-60$

\section{DOSSIER “TintignaC”}

Chr. Maniquet, Th. Lejars, B. Armbruster, M. Pernot, M. Drieux-Daguerre, P. Mora, L. Espinasse

Le carnyx et le casque-oiseau celtiques de Tintignac (Naves-Corrèze). Description et étude technologique

\section{E. ArticA}

Júpiter en los Pirineos. El mundo religioso vasco-aquitano, una aproximación

Ph. Poirier, avec la collab. De A.-M. Fourteau-Bardaji

Contribution des fouilles récentes à la connaissance de l'édifice monumental dit des "thermes" de la rue Arthur Ranc à Poitiers (Vienne).....

\section{Dossier "En SOUvenir de Michel Martinaud"}

V. Mathé, Fr. TAssauX

Avant-propos

\section{R. Chapoulie, V. Mathé}

Sur les pas de Michel Martinaud, géophysicien.

\section{Mathé, M. Martinaud †, P. Garmy, D. Barraud}

L'agglomération antique de Brion à Saint-Germain-d'Esteuil (Gironde). Organisation de l'espace, structures et formes de l'urbanisme. 


\section{Chr. SireIX}

Contribution des prospections géophysiques à la découverte du principal centre potier antique des Bituriges Vivisques : Vayres-Varatedo (Gironde)

S. FARAVEL

L'apport des prospections géophysiques de Michel Martinaud à l'archéologie castrale en Aquitaine

\section{E. BOUBE}

Contribution à l'étude de la villa de Chiragan : mobilier, galettes et décors en verre inédits

\section{E. JeAn-COURRET}

Le Puy-Paulin à Bordeaux : porte possible de l'enceinte antique et maison médiévale des Bordeaux/Puy-Paulin

\section{Cavaillès, Br. Velde}

Le couvent des Cordeliers de Parthenay (Deux-Sèvres) : étude des vitraux et des sépultures

\section{ChroniQue}

\section{CARtron}

Chronique de l'archéologie médiévale du haut Moyen Âge en Aquitaine entre Loire et Pyrénées (2003-2011) $.353-360$

\section{THĖSE}

C. BRIAL, Les décors sculptés à personnages des monuments funéraires en Aquitaine sous le Haut-Empire.

$363-368$ 


\section{Dossier}

\section{Tintignac}




\author{
Christophe Maniquet, Thierry Lejars, Barbara Armbruster, \\ Michel Pernot, Monique Drieux-Daguerre, Pascal Mora, Loïc Espinasse \\ en collaboration avec \\ Frédéric Adamski, Serena Campodonico et Paolo Piccardo
}

\title{
Le carnyx et le casque-oiseau celtiques de Tintignac (Naves-Corrèze). Description et étude technologique
}

RÉSUMÉ

Deux des objets issus d'une petite fosse du sanctuaire gaulois de Tintignac ont fait l'objet d'une restauration et d'un examen approfondi. Il s'agit d'une trompette de guerre gauloise (ou carnyx) et d'un casque en forme d'oiseau. Leur rareté (pour le carnyx) ou leur unicité (pour le casque), comme leur mode de fabrication complexe leur confèrent un caractère exceptionnel. L'approche pluridisciplinaire utilisée combine plusieurs méthodes des sciences humaines et des sciences des matériaux, dont la restauration, l'examen technologique, les études physico-chimiques, les recherches sur les aspects stylistiques, symboliques et socioculturels, et enfin la documentation et l'exposition. Les deux éléments étudiés sont constitués de plusieurs pièces de bronze martelées ou coulées assemblées par brasure ou par emboîtement. L'étude archéométallurgique a permis d'étudier précisément les alliages de bronze qui entraient dans leur composition. La trompette possède au niveau de son pavillon en forme de hure de sanglier, des oreilles démesurées semblables à des feuilles, tandis que le casque prend la forme d'un oiseau dont la posture pourrait évoquer celle d'un cygne. Ces objets extraordinaires pouvaient être utilisés lors des combats ou des cérémonies pratiquées sur le sanctuaire. La volonté des Gaulois d'impressionner l'adversaire en associant une vision frappante à un bruit assourdissant, telle que nous la rapporte les auteurs gréco-romains, est bien illustrée par ces deux objets d'exception.

\section{MOTS-CLÉS}

Carnyx, casque, armement, symbolique, instrument de musique antique, archéométallugie, technologie, animaux, bronzes celtiques, artisanat laténien.

\section{ZUSAMMENFASSUNG}

Zwei Gegenstände, die in einer kleinen Grube des gallischen Heiligtums von Tintignac deponiert waren, wurden restauriert und eingehend untersucht. Es handelt sich um eine gallischen Kriegstrompete (oder carnyx) und um einen Helm in Form des Vogels. Ihre Seltenheit (für die Carnyx) oder ihre Einmaligkeit (für den Helm), wie auch ihre komplexe Herstellung verleihen ihnen einen ungewöhnlichen Charakter. Der multidisziplinäre Forschungsansatz der Untersuchung kombiniert mehrere Methoden der Geistes- und Naturwissenschaften, darunter die Restaurierung, die Untersuchung der technologischen Aspekte, die chemischen Analysen, die Erforschung der stilistischen, symbolischen und soziokulturellen Gesichtspunkte, und schließlich die Dokumentation und die Ausstellung. Die beiden untersuchten Objekte sind aus mehreren geschmiedeten und gegossenen Elementen aus Bronze, hergestellt, die durch Weichlöten und Steckverbindungen zusammengefügt wurden. Die archäometallurgischen Analysen haben die genaue Zusammensetzung der Legierungsbestandteile der Bronze bestimmt. Die Trompete hat am oberen Ende die Form eines Wildschweinkopfes, dessen überdimensionierten Ohren Blättern ähneln, während der Helm die Form eines Vogels hat, dessen Haltung an diejenige eines Schwanes erinnert. Diese außerordentlichen Gegenstände konnten während der Kämpfe oder der auf dem Heiligtum ausgeübten Zeremonien benutzt worden sein. Diese zwei außergewöhnlichen Kultobjekte stellen gut den Wunsch der Gallier dar, den Gegner zu beeindrucken, indem ein erschreckender Anblick mit einem betäubenden Lärm vereint wird, so wie es die griechisch-römischen Autoren berichteten.

\section{SCHLÜSSELWÖRTER}

Carnyx, Archäometallurgie, Helm, Bewaffnung, Symbolik, antike Musikinstrumente, Technologie, Tiere, Celtic Bronze, Crafts Latenian. 

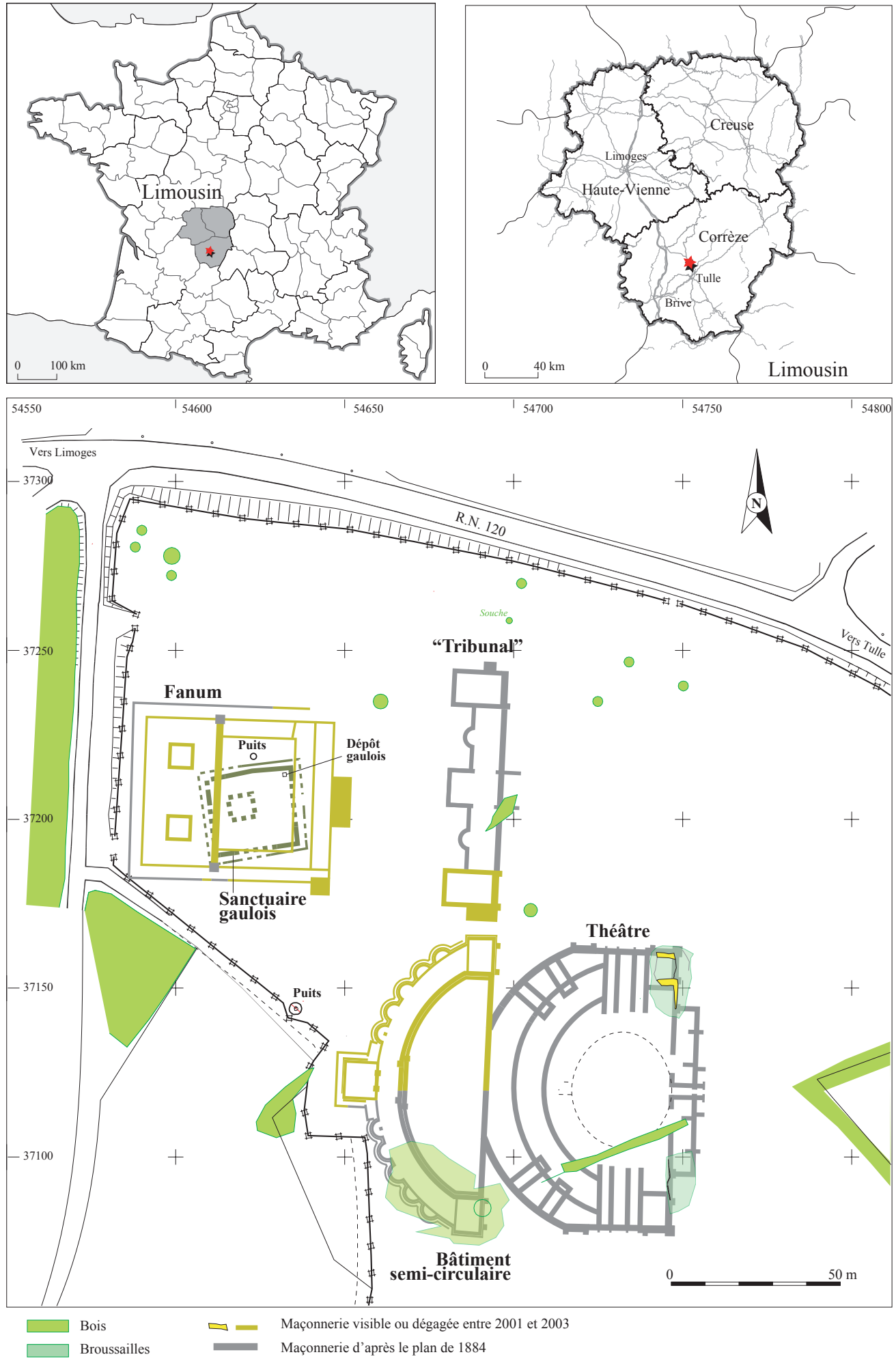

Fig. 1. Plan de l'ensemble monumental cultuel gallo-romain et emplacement du sanctuaire et du dépôt gaulois (relevé Chr. Maniquet, INRAP et Jean-Louis Courtadon). 
Ce dossier se propose de faire le point sur les recherches menées sur les deux premiers objets restaurés extraits $d u$ dépôt d'objets découvert en 2004 dans le sanctuaire gaulois de Tintignac (Naves-19). Archéologues, archéo-métallurgistes, restauratrices, photographes, dessinateurs se sont associés pour extraire de ces objets uniques toutes les informations disponibles. Bien d'autres objets sont en cours ou en attente de traitement. Il a cependant semblé important pour l'équipe de faire un premier point et d'informer de l'état d'avancement de la recherche sur cette fabuleuse découverte.

\section{LE CONTEXTE DE LA DÉCOUVERTE}

(Chr. Maniquet)

Le sanctuaire gaulois de Tintignac a été édifié vers le milieu du $\mathrm{II}^{\mathrm{e}}$ s. a.C. en bordure d'un axe important traversant le territoire lémovice. Reconstruit plusieurs fois, il garde la même morphologie jusqu'à sa destruction dans les dernières décennies avant notre ère. Au début de notre ère, deux fanums sont inclus dans un espace sacré, cerné par un mur péribole, qui intègre la quasi-totalité de la plate-forme du sanctuaire primitif (fig. 1). D'autres édifices sont par la suite adjoints au temple et occupés jusqu'à la fin du III $^{\mathrm{e}}$ s. p.C. ${ }^{1}$.

L'espace sacré gaulois, protégé par une palissade, incluait un édifice de bois (fig. 2) au centre et quelques fosses éparses ${ }^{2}$. Dans l'angle nord-est, directement sous la couche charbonneuse scellant les structures du sanctuaire protohistorique, une petite fosse a été mise au jour. De forme carrée avec des angles arrondis, elle était dotée d'une petite excroissance circulaire au nord-ouest. Plus de 500 fragments d'objets en fer et en alliage cuivreux, appartenant à une soixantaine d'objets la comblaient. Il s'agit essentiellement d'armes (épées, fourreaux, fers de lance, umbo de bouclier, casques), mais également de représentations d'animaux en tôle de bronze, démontées, d'un chaudron et d'instruments de musique (carnyx), sans compter les éléments non identifiés ${ }^{3}$.

\footnotetext{
1. Maniquet 2004.

2. Maniquet 2009b.

3. Maniquet 2005a ; Maniquet 2005b ; Maniquet 2008 ; Maniquet 2009a.
}

\section{Le choix des deux premiers objets}

La fouille et le prélèvement des objets de cette fosse se sont déroulées à l'automne 2004. Après un nettoyage partiel de certains d'entre eux en laboratoire, ils ont été rapidement stockés sous vide partiel.

Les budgets importants nécessaires et les retards rencontrés pour les réunir n'ont pas permis d'entamer l'étude et la restauration de tous les objets rapidement. Ce n'est qu'en début d'année 2008 que les premières études ont pu débuter. Il a été décidé, dans un premier temps, de ne traiter que deux objets, parmi les moins abîmés. Le choix s'est porté sur des pièces présentant une homogénéité quant aux matériaux utilisés, en évitant celles constituées d'assemblages de tôles en fer et en alliage cuivreux ou intégrant des incrustations.

Ainsi ont été retenus le casque le moins écrasé et la trompette de guerre la plus complète. Cette étude devait donner la mesure de leur caractère tout à fait exceptionnel et entraîner une prise de conscience de la part des partenaires financiers et de la communauté scientifique de la nécessité de sauvegarder au plus vite ce fragile patrimoine.

Leur restauration a été accompagnée d'un examen approfondi réalisé par plusieurs spécialistes, dont cet article se veut le compte-rendu. Depuis le début de l'année 2009, les deux premiers objets restaurés ont été exposés à Tulle (Corrèze) et Toulouse (Haute-Garonne) dans le cadre d'expositions consacrées à cette découverte, ou bien, comme à Berne (Suisse), associés à d'autres chefs-d'œuvre de l'art celtique.

La trompette décrite ici est une restitution idéale réalisée à partir de plusieurs éléments découverts dans la fosse. Plusieurs indices semblent confirmer qu'ils ont réellement appartenu à l'origine à la même trompe. Seule l'embouchure ne peut lui être rattachée de façon certaine ; elle permet cependant d'extrapoler la morphologie complète de ces objets.

\section{La place des objets dans le dépôt}

Le casque en forme d'oiseau et le carnyx présentés ici sont les premiers objets déposés dans la fosse. En effet, tous les éléments y avaient été consciencieusement rangés : les armes en fer, en surface, reposaient sur les portions d'animaux en tôles 


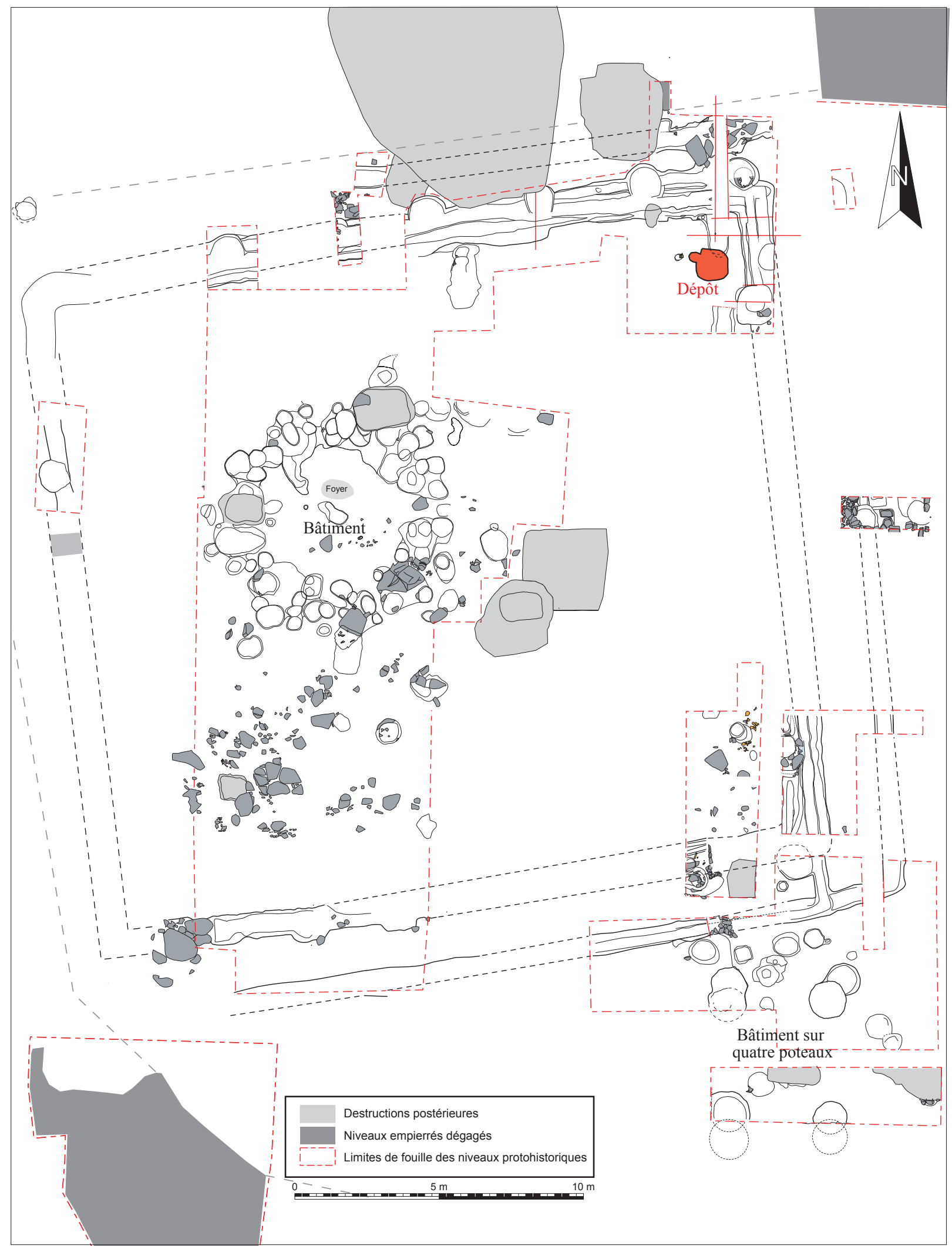

Fig. 2. Plan du sanctuaire gaulois (relevé Chr. Maniquet, INRAP). 


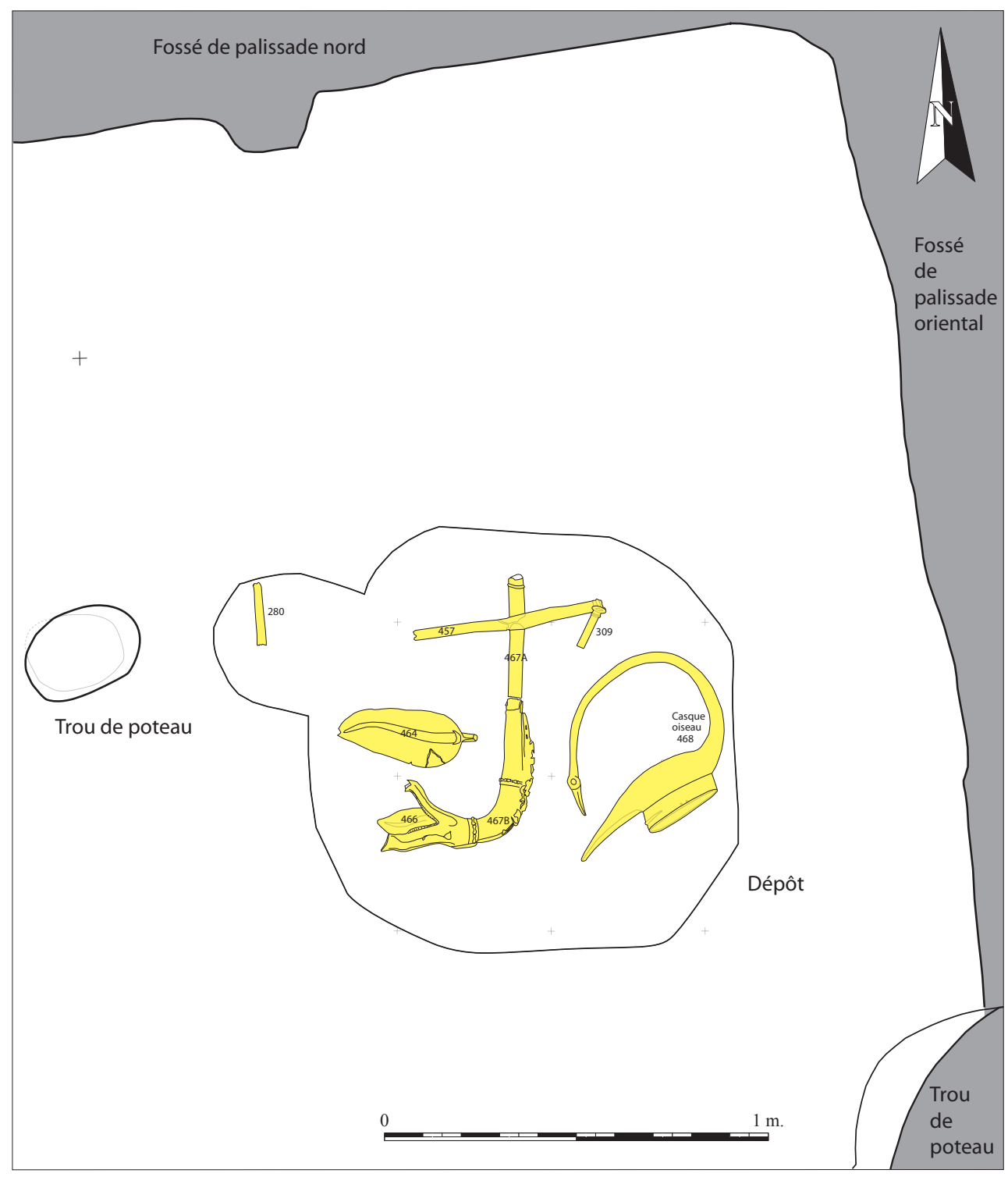

Fig. 3. Plan du dépôt gaulois et des objets restaurés (relevé Chr. Maniquet, INRAP).

de bronze ; en dessous, les casques recouvraient les portions de carnyx. La plupart des pièces, et les armes en particulier, avaient souffert de mutilations volontaires. Les casques avaient été en général littéralement écrasés, celui en forme d'oiseau étant le moins abîmé. En ce qui concerne les trompettes, il n'est pas certain qu'elles aient reçu des coups. Elles ont été soit soigneusement démontées, soit scindées par pliages successifs, pour pouvoir les placer dans la fosse aux dimensions restreintes.

Dix casques ont été identifiés dans le dépôt ${ }^{4}$. L'un d'entre eux possédait une calotte en fer, avec plaques et cimier décorés selon le style plastique.

4- Maniquet 2008. 


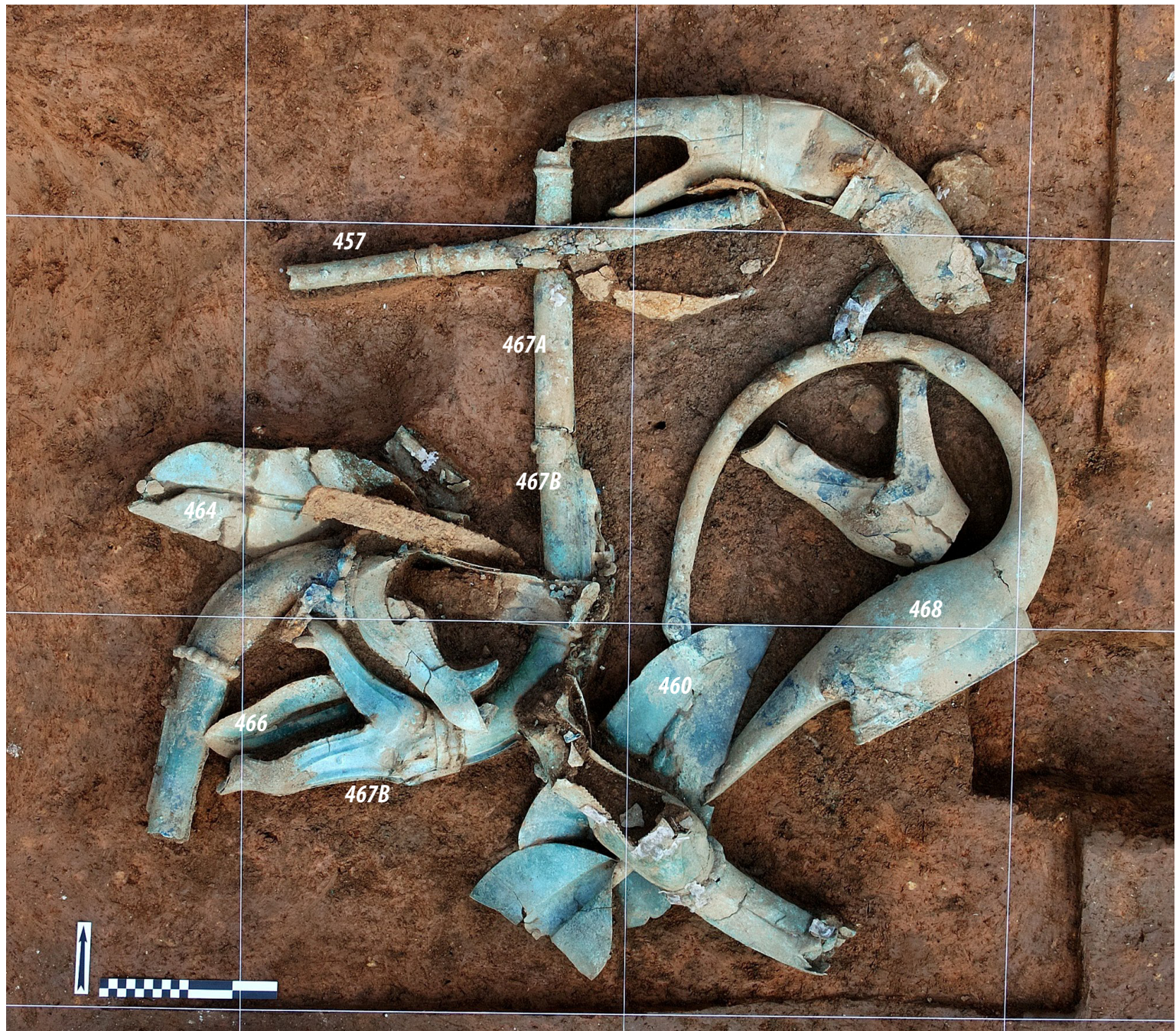

Fig. 4. Le niveau inférieur des objets reposant dans la fosse. Ne restent plus que les trompettes de guerre et le casque-oiseau. Au centre et pavillon vers le bas, se trouve le carnyx traité ici et à droite, le casque-oiseau (cl. Patrick Ernaux, INRAP).

Tous les autres étaient constitués de pièces en alliage cuivreux assemblées. Parmi ces casques, l'un était doté d'une résille ajourée en partie supérieure et antérieure ; un autre disposait de trois grands anneaux disposés en triangle. Tous, hormis le casque-oiseau et celui en fer, étaient pourvus à l'origine de paragnathides et d'un couvre-nuque rivetés sur le timbre.

Le casque-oiseau ( $\left.n^{\circ} 468\right)$ et la partie de la trompe dotée du pavillon-sanglier $\left(\mathrm{n}^{\circ} 467\right)$ reposaient à plat sur le fond de la fosse, respectivement à l'est et à l'ouest (fig. 3 et 4). Les deux "animaux" étaient dé- posés sur leur flanc gauche et regardaient l'un comme l'autre vers l'ouest. Alors que le pavillon du carnyx se trouvait au sud, le cou de l'oiseau se développait vers le nord. Le carnyx semble avoir été posé d'abord au centre de la fosse, avant le casque placé dans l'espace libre à l'est.

Le couvre-chef reposait simplement sur un fragment de fourreau en fer et un tube de carnyx avait été glissé à l'intérieur du timbre. Un pavillon de carnyx démonté a été soigneusement placé dans l'enroulement du cou de l'oiseau, tandis qu'une tôle de 


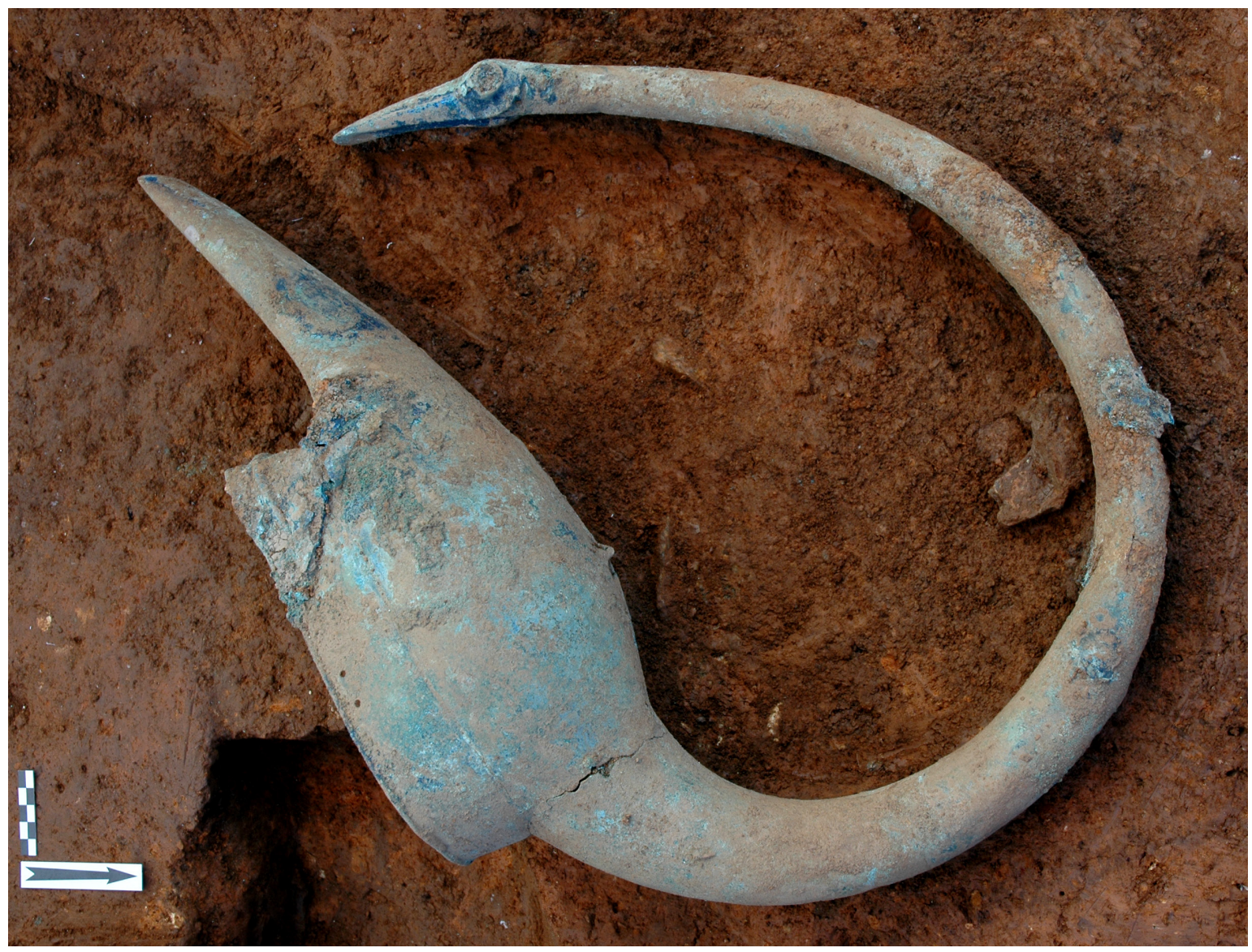

Fig. 5. Le casque-oiseau reposant au fond de la fosse (cl. P. Ernaux, INRAP).

métal en forme de feuille (correspondant à l'origine à une oreille de trompette) est venue dissimuler partiellement sa tête (fig. 4). Le casque doté de trois anneaux déposé à plat sur le casque-oiseau, le recouvrait totalement.

La portion de carnyx comprenant le pavillon, la partie courbe du tube, avec la crête, et une partie du tube rectiligne, reposait à plat sur le fond de la fosse $\left(n^{\circ} 467\right)$. Elle était fissurée en deux, juste en-dessous de la base de la crête (fig. 4). Le tube droit distal a été enregistré en $n^{\circ} 467 \mathrm{~A}$ et celui doté du pavillon en $n^{\circ} 467 \mathrm{~B}$.

L'extrémité du tube $\mathrm{n}^{\circ} 467 \mathrm{~A}$ disparaissait sous le casque $\mathrm{n}^{\circ} 424$ à l'intérieur duquel un fragment ( $\left.n^{\circ} 474\right)$ a été retrouvé lors du nettoyage en labora- toire. Ce dernier recollait parfaitement à l'extrémité du tube $n^{\circ} 467 \mathrm{~A}$.

Une autre portion $\left(\mathrm{n}^{\circ} 457\right)$ reposait sur l'élément $n^{\circ} 467 \mathrm{~A}$, perpendiculairement à ce dernier (fig. 4). D'autres morceaux de tubes de carnyx ont été mis au jour dans le dépôt, dotés ou non de bagues de raccord de morphologie ou de section différente. Le seul possédant des bagues de raccord moulées identiques à celles du n467A est précisément cette section de tube $\mathrm{n}^{\circ} 457$. Bien qu'aucune liaison physique n'ait pu être confirmée, il est très probable que ces tubes aux diamètres différents aient appartenu au même instrument. C'est donc avec une forte présomption que ces deux portions ont été rassemblées pour la reconstitution proposée ici. 
Le tube $n^{\circ} 280$, quant à lui, a été retrouvé dans l'extension circulaire, en bordure occidentale de la fosse (fig. 3), bien au-dessus des autres fragments de trompes, entre les tôles d'animaux en bronze et les armes en fer, quasiment au même niveau que l'embouchure. Là encore, c'est lors de leur nettoyage en laboratoire que le lien physique entre ce tube et l'une des extrémités du tube $n^{\circ} 457$ a pu être mise en évidence.

La seule embouchure du dépôt (n³09) a été mise au jour dans le quart nord-est de la fosse, avec d'autres petits fragments de trompe, juste sous les fourreaux et lames d'épée superficiels (fig. 3). Cette embouchure, complète, n'était en connexion avec aucun tube. Il est impossible de savoir à quel instrument elle a appartenu. Elle a été prise en compte pour présenter un instrument complet mais rien ne permet de l'associer avec certitude avec l'instrument présenté ici.

Les deux tôles en forme de feuille mises au jour sur le terrain à proximité immédiate du pavillon 467B ont été restaurées (fig. 4). En aucun cas, tout comme pour l'embouchure, il n'est assuré qu'elles ont fonctionné avec cet instrument.

La première $\left(\mathrm{n}^{\circ} 466\right)$ reposait à plat sur le fond de la fosse, sous le pavillon. La seconde ( $\left.{ }^{\circ} 464\right)$, complète, se trouvait à peu de distance au nord de notre pavillon et sous le pavillon d'un autre carnyx. Une troisième ( $\left.\mathrm{n}^{\circ} 460\right)$, quasiment entière aurait pu être prise en compte. Elle reposait aussi sur le fond de la structure, sous le pavillon qui conservait son oreille en connexion, et sur la tête du casque-oiseau. Malheureusement elle était moins bien conservée que les précédentes et sa restauration ne pouvait être réalisée dans des délais raisonnables. C'est la plus grande oreille mise au jour dans le dépôt, avec une longueur de $410 \mathrm{~mm}^{5}$.

Lors du montage de l'oreille $\mathrm{n}^{\circ} 464$ sur le pavillon $\mathrm{n}^{\circ} 467$, la parfaite adaptation de l'une dans l'autre laisse imaginer que ces éléments ont bien fonctionné ensemble même si rien ne permet de l'assurer. Il semblait important, pour avoir une vision complète de la trompe gauloise, de la pourvoir d'au moins un de ses appendices auriculaires.

5- Maniquet 2008, 308.
Le carnyx présenté ci-dessous a donc été reconstitué à partir d'éléments issus, peut-être, de plusieurs instruments. Cependant, une forte probabilité existe quant à l'appartenance de tous ces fragments au même objet à l'origine, hormis l'embouchure et l'oreille 466.

Le casque quant à lui a été retrouvé en une seule pièce et n'a fait l'objet d'aucun remontage postfouille (fig. 5). Intact dans le dépôt, il a été simplement vidé du sédiment qu'il renfermait, nettoyé et restauré.

\section{LE CARNYX}

\section{Introduction \\ (Chr. Maniquet)}

Les textes classiques, peu nombreux, évoquant la trompette celtique et son bruit assourdissant, ne décrivent pas du tout sa morphologie ${ }^{6}$. Cette dernière est connue en fait par ses nombreuses représentations, sur des monuments romains ou gallo-romains, ou sur des monnaies gauloises, républicaines et même, dans une moindre mesure, impériales. Il s'agit d'une trompe de la hauteur d'un homme dont le pavillon prend la forme d'une tête d'animal plus ou moins fantastique (le sanglier semblant cependant l'animal de prédilection) par la gueule ouverte duquel le son est expulsé. Seuls quelques fragments de carnyx ont été découverts au travers du monde celtique ${ }^{7}$. La fosse de Tintignac recelait les pavillons de sept trompes, dont deux en bronze moulé et cinq en tôles martelées assemblées. Ces pavillons se terminent tous par une hure de sanglier plus ou moins stylisée, sauf un qui s'apparente davantage à un serpent.

Le carnyx présenté ici est constitué de tôles de bronze et doté d'une hure de sanglier. Il était à l'ori-

\footnotetext{
6- "Les Romains étaient frappés par l'arrangement et les bruits confus de l'armée des Celtes; innombrable y était la foule des sonneurs de cors et de trompettes; et en même temps toute l'armée entonnant son chant de guerre, il en résultait une si grande, une si formidable clameur que, non seulement les trompettes et les troupes mais encore les lieux voisins résonnant de concert semblaient eux-mêmes pousser des cris" (Pol., Hist., 2.29). "Ils ont des trompettes d'une nature particulière et bien faite pour des Barbares: ils soufflent dans ces trompettes et en font sortir un son rude qui convient bien au tumulte de la guerre" (Diod., Bibliothèque historique, 5.30.4).

7- Le carnyx et la lyre, 1993 ; Hunter 2001.
} 


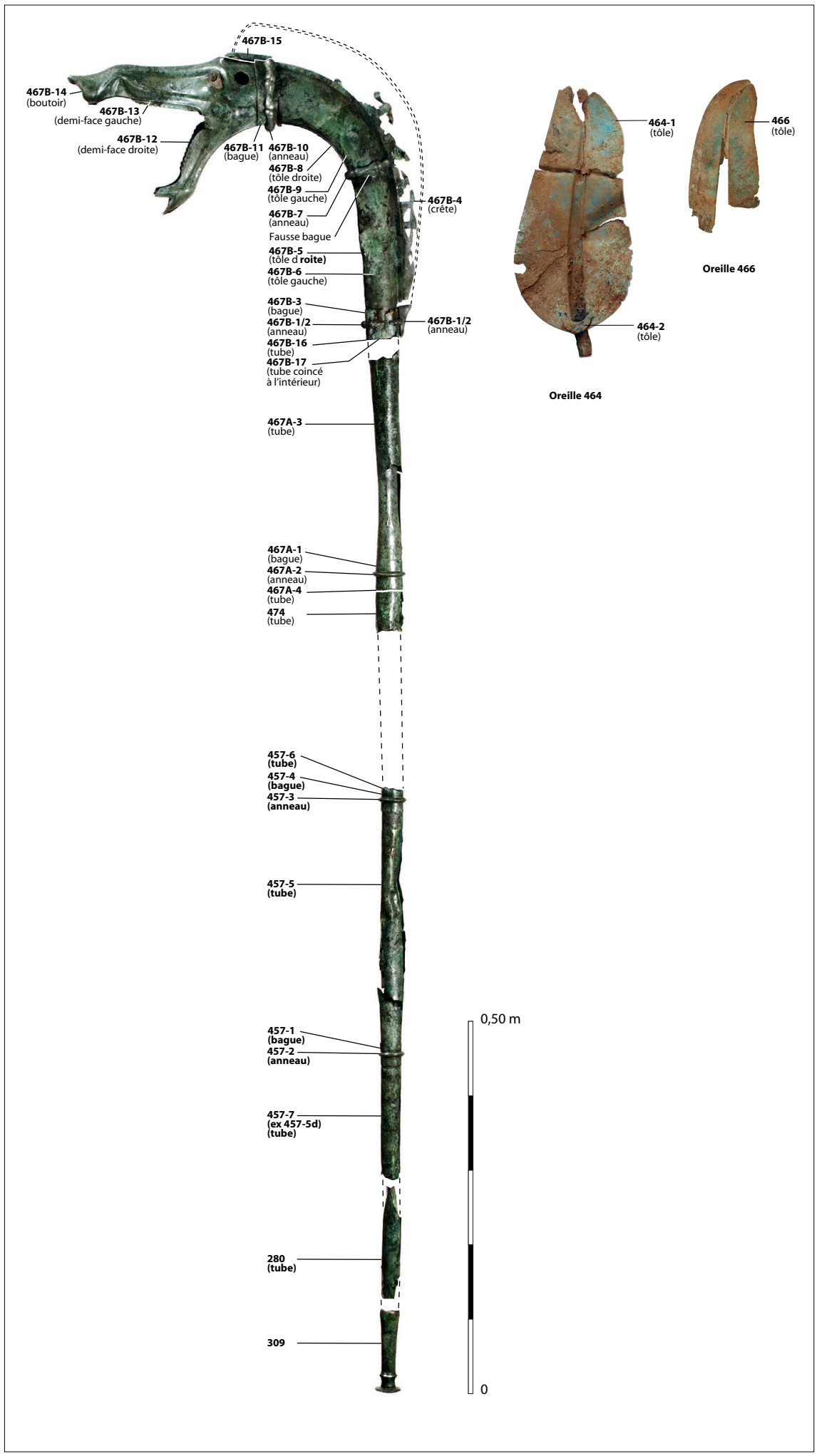

Fig. 6. Le carnyx reconstitué avec numéro d'inventaire de chaque élément constitutif (cl. P. Ernaux, INRAP). 


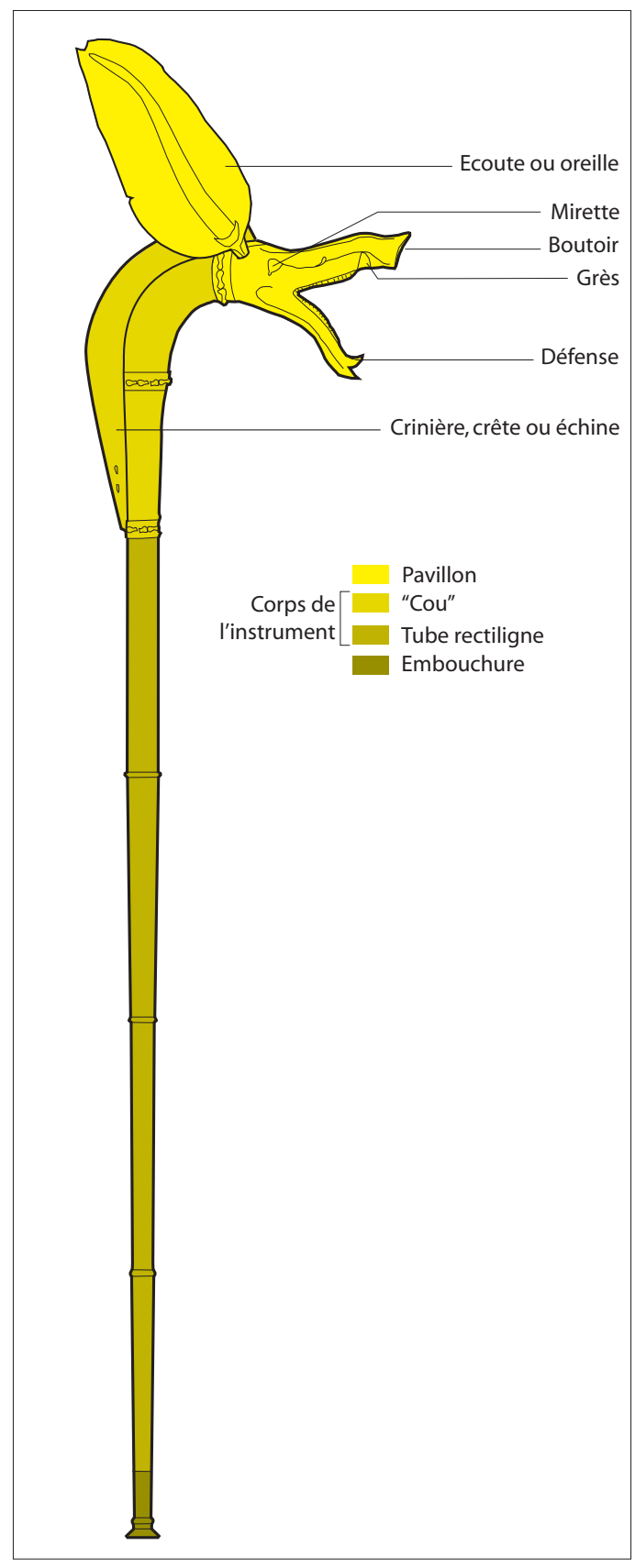

Fig. 7. Dénomination des différentes parties d'un carnyx (dessin Chr. Maniquet, INRAP). gine constitué de 28 pièces métalliques distinctes assemblées (fig. 6), dont 25 ont été retrouvées dans le dépôt (manquent une oreille et sa lunule, et l'ourlet de la crête). L'instrument peut être divisé en trois parties distinctes : le pavillon représenté par l'extrémité évasée de l'instrument (soit la hure de sanglier à la gueule grande ouverte), le corps de l'instrument et l'embouchure. Le corps peut à son tour être subdivisé en 2 parties : le "cou", matérialisé par la partie courbe du tube associée à la crête, et la partie inférieure, rectiligne, du tube (fig. 7).

\section{Description \\ (Chr. Maniquet)}

Le pavillon

Le pavillon de l'instrument regroupait à lui seul sept tôles dont cinq ont été retrouvées (fig. 6) : trois pour la hure (deux demi-faces et le boutoir) et deux pour la seule oreille qui a pu lui être rattachée.

La hure

Le pavillon de l'instrument de musique est représenté sous la forme de la gueule béante d'un animal stylisé (fig. 8). Certaines caractéristiques (dents, boutoir) permettent cependant de le rapprocher du sanglier, animal emblématique gaulois par excellence, symbole de puissance et de courage, qualités tant recherchées par les membres de l'aristocratie guerrière.

La hure proprement dite est composée de deux tôles martelées pour chacune des demi-faces et d'une autre plus petite pour le boutoir. À l'opposé de ce dernier, les deux tôles formaient un cylindre de $83,6 \mathrm{~mm}$ de diamètre pour être assemblés avec le tube du cou de l'animal.

- Les demi-faces

Les deux demi-faces, dont la bordure distale disparaissant sous la première bague du cou (fig. 9 et 10), ne se joignaient pas bord à bord et conféraient encore au pavillon une largeur maximale, d'une joue à l'autre, de $82 \mathrm{~mm}$, malgré un écrasement visible.

La face droite (467B-12) recouvre de quelques millimètres la gauche (467B-13) sous la mandibule, en dessous du groin et au sommet de la hure. Ailleurs, c'est la tôle gauche qui se superpose à la 


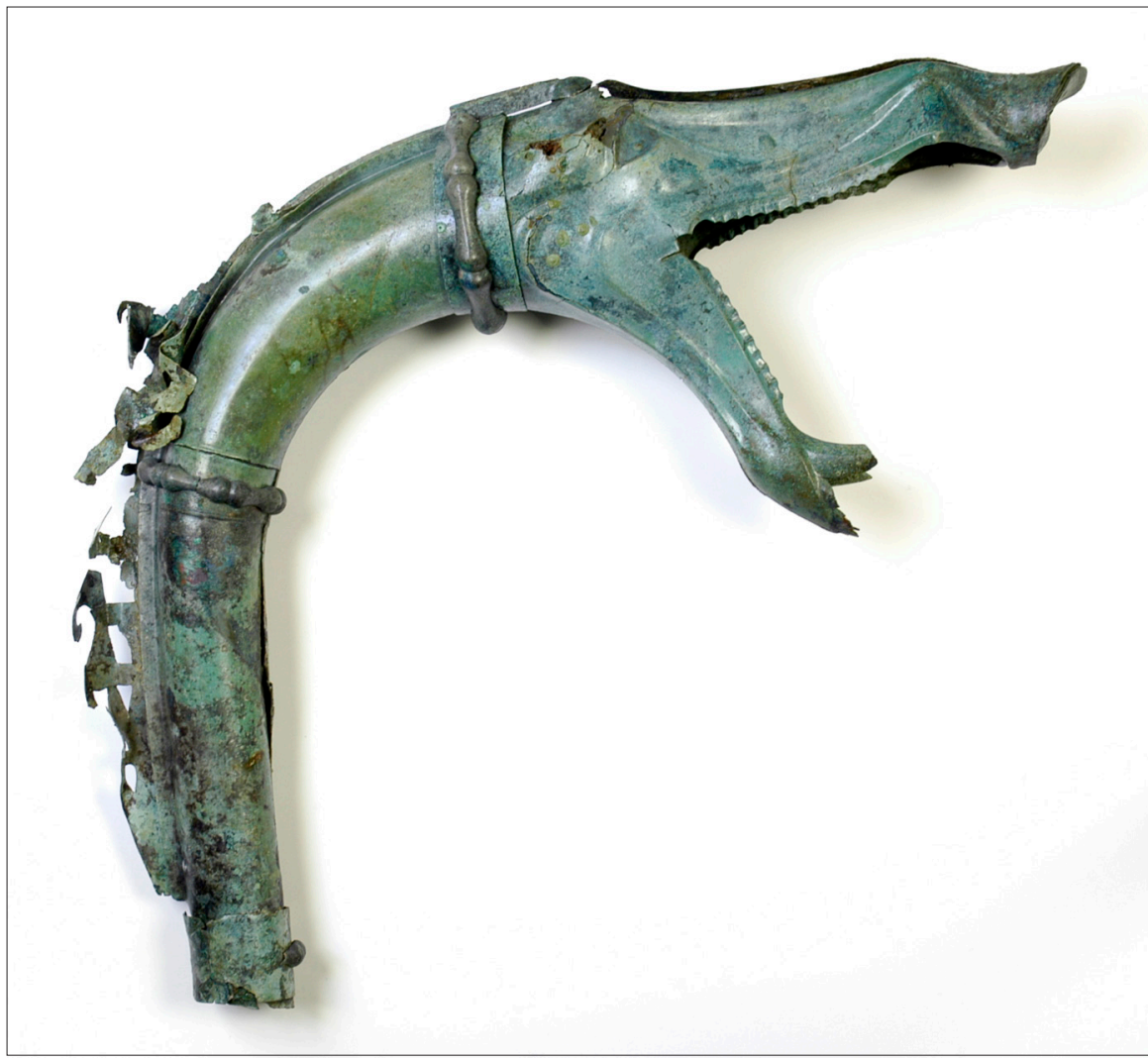

Fig. 8. Le pavillon du carnyx restauré (cl. B. Armbruster, CNRS).

droite (jusqu'à $12 \mathrm{~mm}$ au-dessus des yeux). Les deux tôles sont désolidarisées en partie supérieure, la gauche semblant s'être soulevée suite à son enfoncement. Ce pavillon de carnyx reposait au fond de la fosse sur le côté gauche ; le poids des terres a donc favorisé un aplatissement de la tôle droite qui s'est partiellement emboîtée dans la gauche. Ces pièces étaient à l'origine assemblées bord à bord, sans renfort interne (voir infra, Les aspects technologiques de B. Armbruster).

Une ligne en relief travaillée au repoussé joint le boutoir au sourcil, contourne l'œil et se prolonge à l'arrière jusqu'à la bague 467-B11 qui cache la jonction avec le cou. Elle délimite un front plat et lisse. Une autre ligne prend naissance à la base du groin, contourne le grès, passe sous l'œil, disparaît presque au niveau de la pommette, contourne la joue, longe la mâchoire inférieure et rejoint la défense.

Une troisième ligne débute au-dessous de l'oreille près de l'extrémité de la première, forme une courbe pour marquer le contour de la mandibule et se poursuit de façon rectiligne à la base de cette dernière, presque jusqu'à son extrémité. Une dernière ligne enfin contourne toute la gueule et délimite les dents.

Les yeux (ou mirettes) étaient marqués par une cavité peu profonde, de forme triangulaire. L'œil gauche, contrairement à l'œil droit qui était vide, conservait sa pupille en lignite entourée d'un alliage métallique oxydé gris, teinté de vert, d'aspect granuleux et friable (fig. 11). Prenant naissance à l'angle médial de l'œil, un larmier représenté en relief se termine en forme de goutte (fig. 9 et 10$)^{8}$.

8- Ce type de glandes préorbitaires existe bien chez le sanglier, tout comme chez le cerf où il est plus développé. On note une volonté de représenter de façon très discernable ce larmier qui ne constitue pas une larme de pleur. Ces glandes phéromonales permettent aux animaux, au même titre que leurs cris, de se reconnaitre, de communiquer entre eux ou encore de marquer leur territoire. N'est-ce pas aussi le rôle des carnyx?

D'après le comte de Buffon, le larmier du cerf se retrouve, moins développé certes, entre autres, chez le daim, la biche ou le sanglier. “...au-dessous de l'angle antérieur de chaque oeil du cerf, une cavité (...) s'ouvre au dehors par une fente (...) dirigée en ligne droite du côté de la commissure des lèvres (...). On donne à ces cavités le nom de larmiers, et à la matière qu'elles contiennent, celui de larmes, ou de bézoard de cerf (...) ; l'humeur qui suinte de ses parois, restant dans cette cavité, y forme une matière de même nature que la cire des oreilles" (Leclerc 1756, 109). 


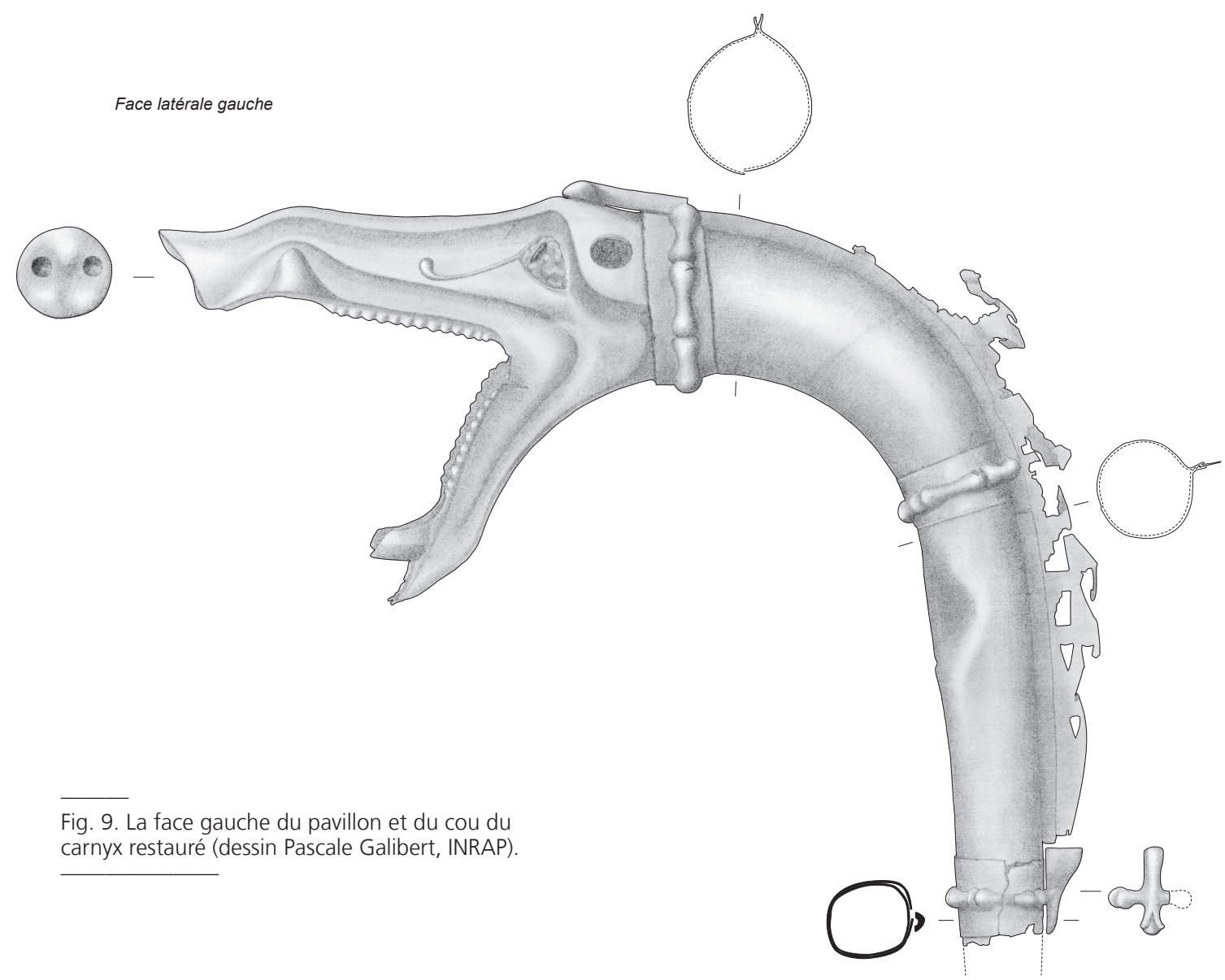

.......

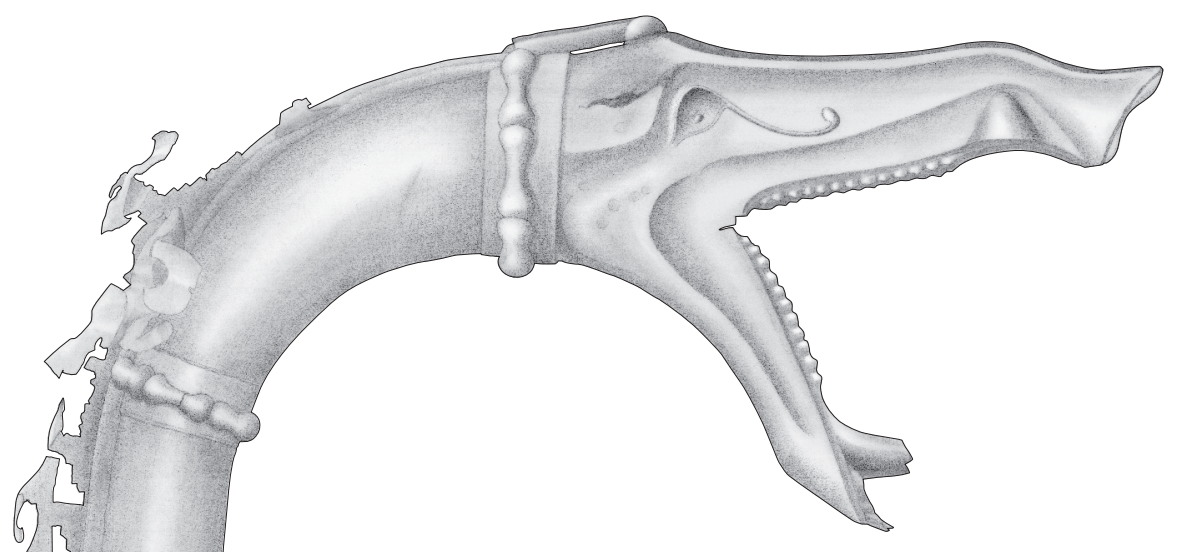

Face latérale droite

Fig. 10. La face droite du pavillon et du cou du carnyx restauré (dessin P. Galibert, INRAP). 


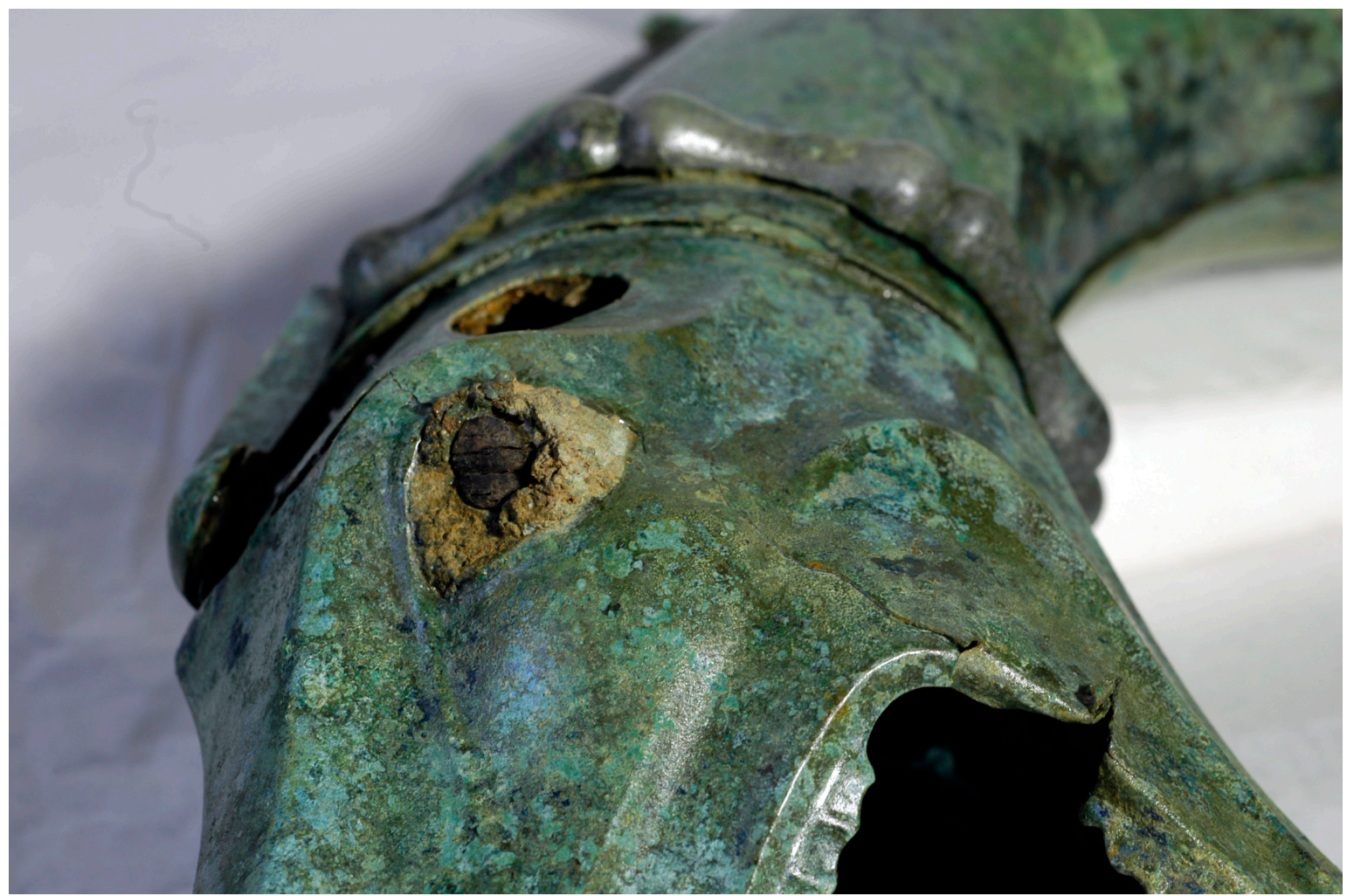

Fig. 11. L'œil gauche du carnyx (cl. B. Armbruster, CNRS).

À l'arrière des yeux, des trous recevaient initialement des oreilles (ou écoutes) qui n'étaient plus en place lors de l'enfouissement dans le dépôt. Au niveau du profil gauche, cette perforation est parfaitement circulaire ; à droite, elle est déformée par un coup porté au-dessus de l'œil (alors que l'oreille n'était déjà plus en place).

En ce qui concerne les dents, l'extrémité de la mâchoire inférieure est dotée sur chaque demi-face de trois incisives et d'une défense. Leur représentation est assez réaliste avec une position fortement inclinée vers l'avant (fig. 12). Le cumul avec l'autre demi-face représente six incisives au total ${ }^{9}$.

9. Ce qui est effectivement le cas du sanglier alors que les cervidés en possèdent huit. Cependant, on notera que sur nos hures de métal, aucune incisive n'est représentée sous le boutoir, au niveau du maxillaire, ce qui est le cas justement des cervidés.

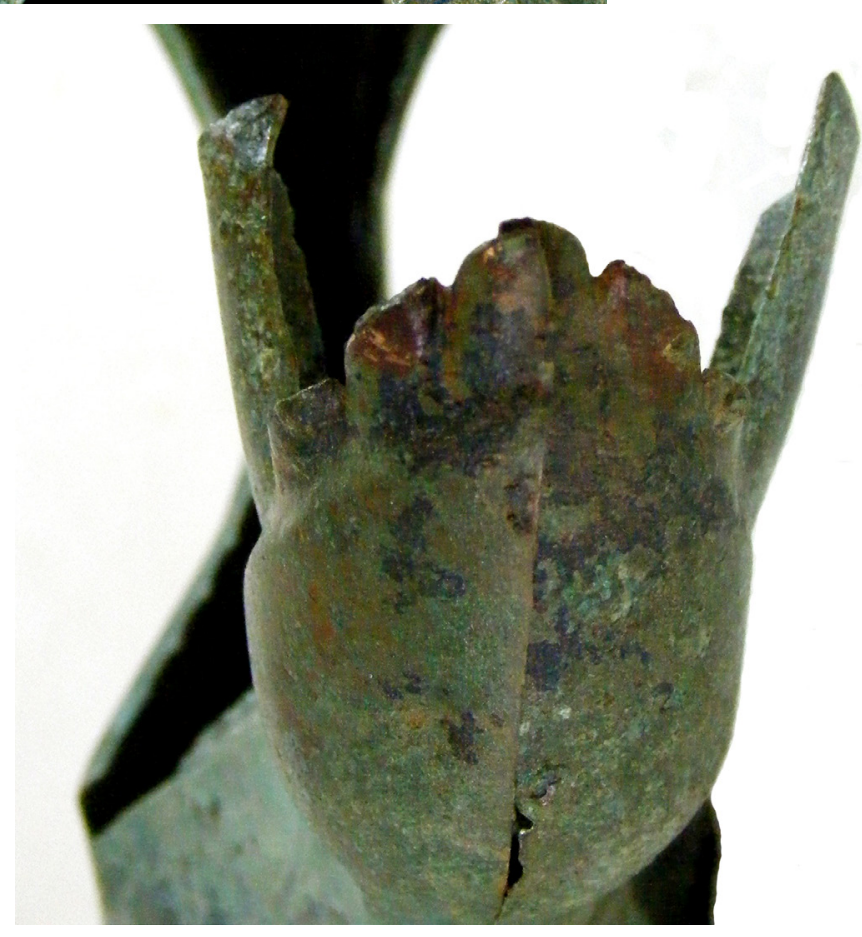

Fig. 12. L'extrémité de la mâchoire inférieure vue de face, avec les incisives inclinées vers l'avant (cl. Chr. Maniquet, INRAP). 
La canine supérieure (ou grès) est simplement rendue en léger relief de forme triangulaire par déformation de la tôle. Les défenses résultent, quant à elles, de découpes spécifiques. Pourvues d'une nervure médiane et en forme de trapèze, elles ne sont pas brisées ni pointues ${ }^{10}$. Les molaires et prémolaires sont en revanche totalement stylisées (fig. 13). On peut en compter, à gauche, 10 en bas et 12 en haut et, à droite, 10 en bas et 13 en haut ${ }^{11}$.

Au total, notre hure possède donc six incisives, quatre canines et 45 déformations correspondant aux prémolaires et molaires.

En ce qui concerne les mutilations volontaires, on a pu noter que sur le côté droit, un enfoncement important au-dessus du trou de l'oreille pourrait ré- sulter d'un coup qui a entraîné une fissuration importante au-dessus des yeux, entre ceux-ci et l'emplacement des oreilles. Ce coup a pu être à l'origine également d'une autre fissure linéaire à l'emplacement de l'angle de la mandibule, joignant la première bague du cou jusqu'à l'œil.

À la commissure de la bouche, sur chacune des deux demi-faces, deux petites entailles espacées de $6,5 \mathrm{~mm}$ et longues de 9 à $20 \mathrm{~mm}$, semblent délibérées (fig. 13), mais leur facture grossière indique qu'elles n'ont pas été réalisées lors de la fabrication de l'instrument, mais plus certainement lors d'une phase ultérieure. S'agit-il de mutilations ? Ces entailles ont-elles eu un rôle fonctionnel, comme la suspension de l'instrument, ou la fixation d'un élément en travers de la gueule de l'animal ?

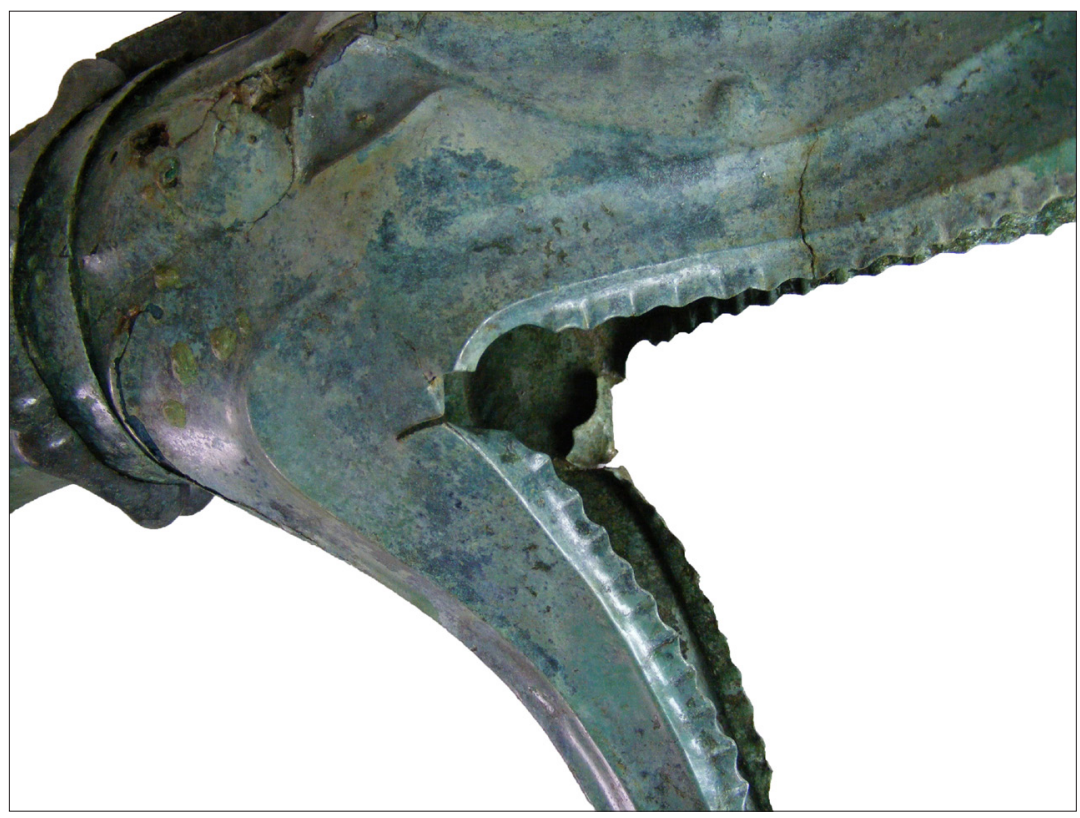

Fig. 13. Des entailles apparaissent de part et d'autre, aux commissures de la bouche de l'animal (cl. Chr. Maniquet, INRAP).

10. Chez le sanglier, la croissance des canines est continue et à développement progressif extérieur aux gencives. Les défenses s'usent sur les grès. C'est sans doute ce qu'on a voulu représenter ici. En règle générale, les défenses sont apparentes hors des lèvres dès la deuxième année. D’après la longueur des défenses et des grès, c'est un mâle de plus de deux ans qui a été représenté mais pas un vieil animal (moins de 4 ans). Il est dans la force de l'âge, à maturité sexuelle et à sa puissance maximale.
11- L'artiste a visiblement martelé les dents sous une forme simplifiée, sans les compter au préalable. En réalité, un sanglier de plus de deux ans possède 12 incisives, 4 canines, 16 prémolaireset 12 molaires, soit 44 dents en tout. Sur chaque demi-mâchoire doit donc se trouver 3 incisives, 4 prémolaires et 3 molaires. Or les trois molaires sont trilobées et trois des prémolaires bilobées, ce qui donne de loin l'illusion d'une quinzaine de dents indépendantes, ce qu'a voulu représenter ici l'artisan. On sait en outre que le pavillon est destiné à être observé depuis une certaine distance qui permet de ne pas s'attarder sur les détails, mais qui oblige à surdévelopper des éléments que l'on désire rendre évidents. 
Sur le côté gauche, la tôle entre l'œil et le grès a subi une légère déformation, sans qu'il soit possible de savoir si elle correspond à un coup. Liées à des dégradations plus "naturelles", à droite, une petite fissure verticale apparaît au niveau du maxillaire et une autre suit la ligne qui contourne la mandibule, près de la bague du cou. On notera, côté droit toujours, la présence de "bubons" de corrosion sous l'oeil, à l'intérieur de sa cavité et autour de l'oreille.

\section{- L'élément 467B-15}

Sur le sommet du crâne, couvrant la jonction des deux profils, mais aussi la bague 467B-11 qui les enserre, une petite pièce accueillait à l'origine l'extrémité antérieure de la crête (fig. 14). Cette petite pièce est constituée d'une tôle d'alliage cuivreux repliée sur elle-même et prend la forme à une extrémité d'une cupule arrondie et creuse. Elle accueillait sans aucun doute l'ourlet (disparu) bordant initialement la crête. Une tôle brisée, mobile, est restée prisonnière entre les deux lamelles de notre élément et provient probablement de la base cassée de cette crête. Une pièce identique a été découverte sur le pavillon du carnyx 463.

\section{- Le boutoir 467B-14}

Le boutoir n467B-14 était attenant mais désolidarisé du groin. Cette simple tôle lisse, presque circulaire, possède de légers reliefs (une bosse dans la partie supérieure et deux autres dans la partie basse). En outre, deux cupules circulaires symbolisant les narines, le rendent très réaliste (fig. 15). Brasée à l'origine directement, bord à bord, avec les profils, sans pièce de renfort, elle était légèrement inclinée vers l'avant. Cet élément conserve une légère pliure médiane.

\section{Les oreilles}

La découverte de Tintignac permet d'affirmer, contre toute attente, que certaines trompettes de guerre gauloises étaient affublées d'oreilles démesurées en tôle. Cinq ont été mises au jour dans le dépôt ; une seule était encore en connexion avec un pavillon de carnyx ${ }^{12}$.

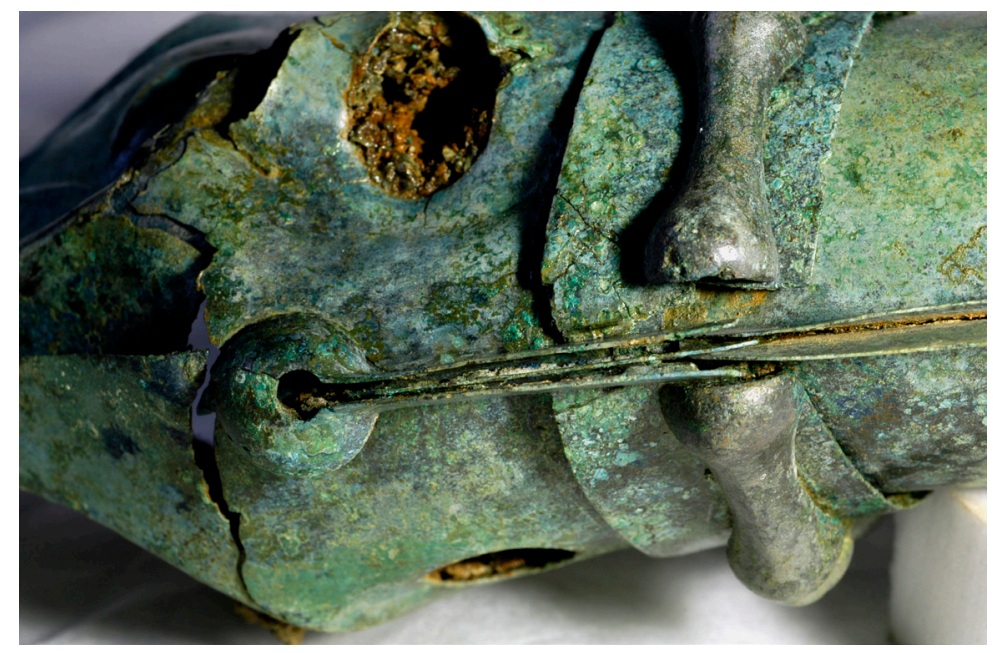

Fig. 14. La face supérieure du pavillon du carnyx. La petite pièce 467B-15 recevait l'extrémité antérieure de la crête (cl. B. Armbruster, CNRS).

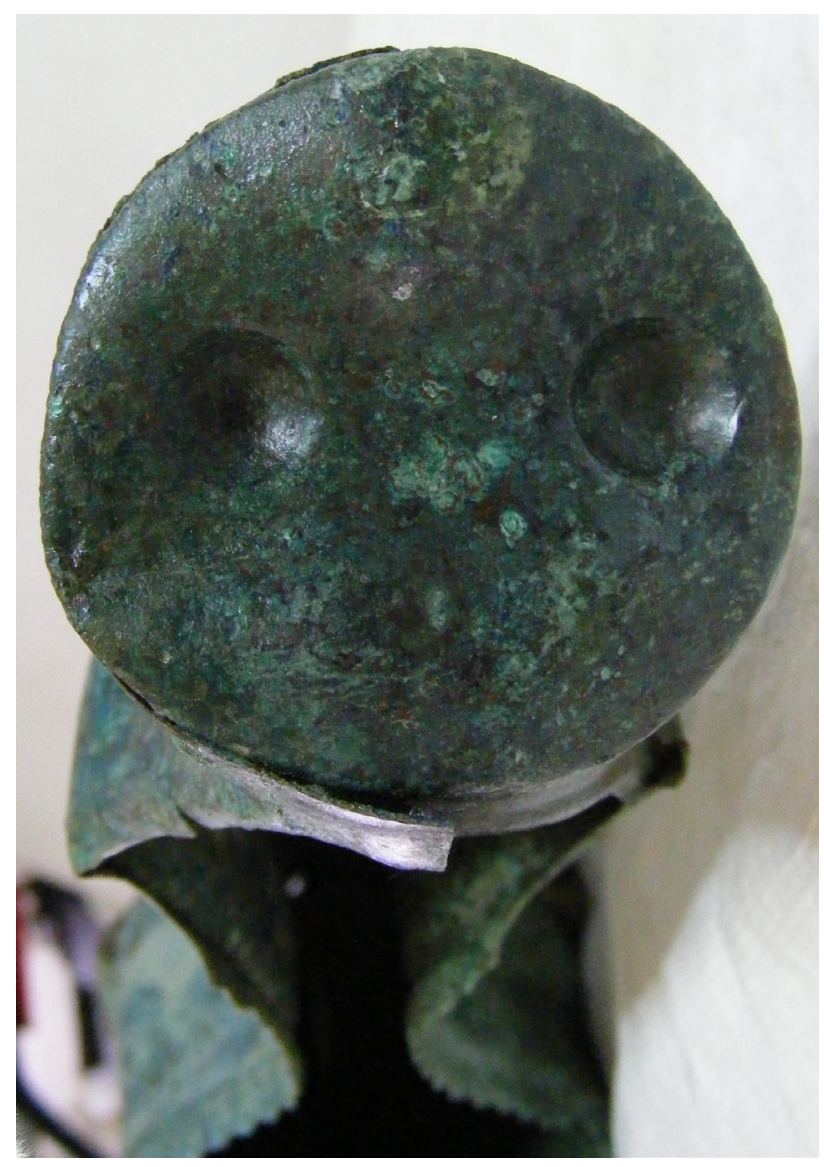

Fig. 15. Le boutoir est matérialisé par une tôle circulaire simplement brasée aux deux demi-faces de l'animal (cl. Chr. Maniquet, INRAP). 


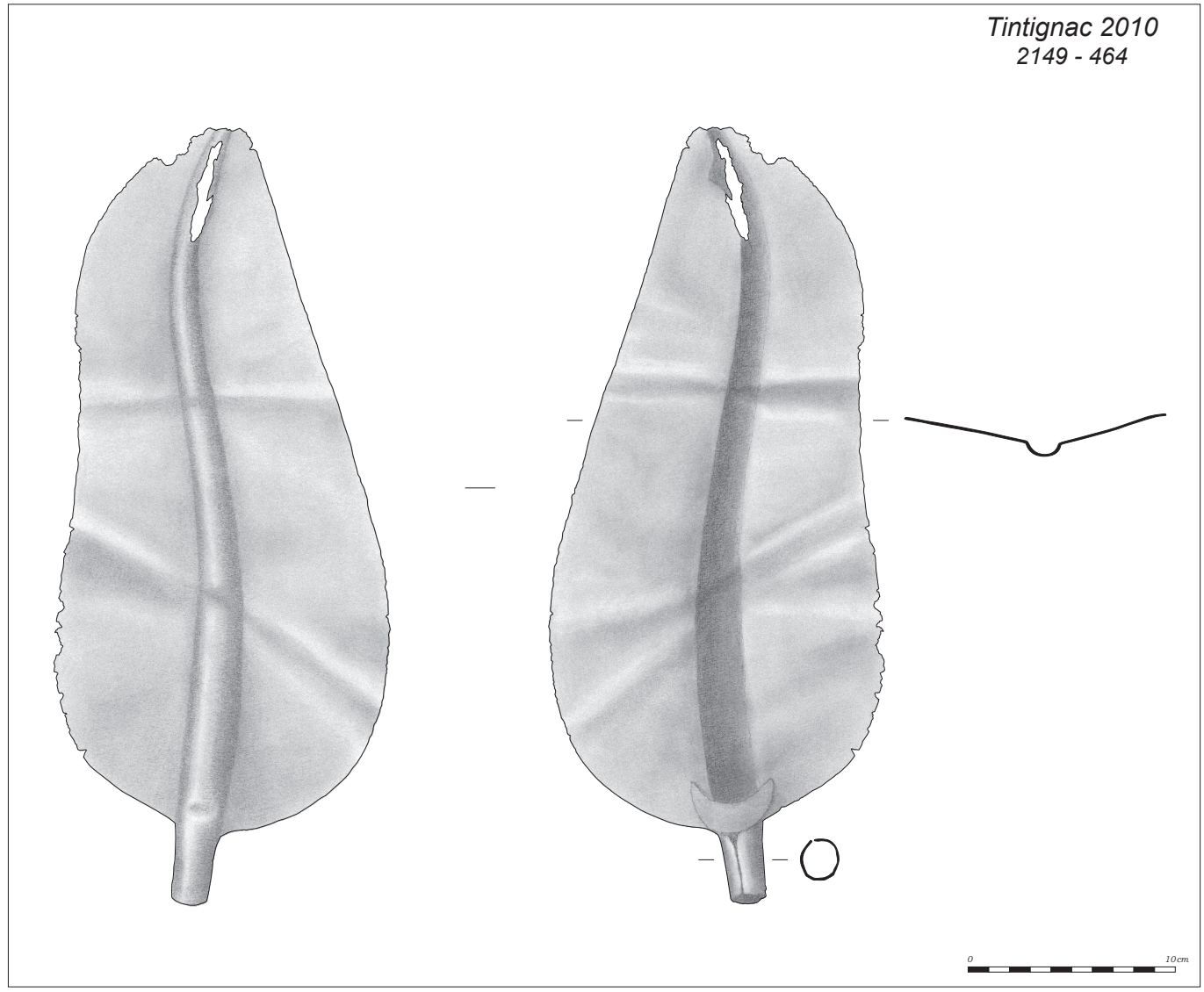

Fig. 16. L'oreille 464 (dessin P. Galibert, INRAP).

Ces oreilles sont constituées de tôles découpées selon la forme d'une feuille d'arbre simple, oblongue, à bord lisse et à base symétrique ${ }^{13}$. L'un des bords du limbe est plutôt rectiligne, tandis que l'autre est plus sinueux. À l'emplacement de la nervure médiane, une gorge rectiligne (visible en creux quand la forme générale de la tôle présente un profil concave), au profil semi-circulaire, s'incurve vers l'extrémité. À l'extrémité proximale, à l'emplacement du pétiole, la tôle est enroulée sous la forme d'un tube qui pénétrait partiellement dans le pavillon.

13. Cette morphologie de feuille est difficile à rapprocher d'un type existant.
L'oreille 464 a été restaurée car c'est la seule complète ; seule l'extrémité distale est abîmée (fig. 16). Son poids total atteint $175,2 \mathrm{~g}$. Une petite pièce métallique (464-2) en forme de lunule a été fixée, en partie supérieure de la douille et couvrant partiellement la gorge médiane.

Cette feuille est marquée de deux grandes fissures transversales. Dans le cadre du rituel de mutilation, elle semble avoir été pliée en trois, la partie distale ramenée contre la face interne et la partie proximale vers l'extérieur. La tôle a ensuite été dépliée et remise à plat, avant son dépôt. Elle s'est ensuite encore déformée sous le poids du pavillon de l'un des autres carnyx. 
Le "cou”

Le cou de l'instrument, haut au total de $365 \mathrm{~mm}$ et large de $213 \mathrm{~mm}$ (vu de profil, de la base de la bague 467-B3 au bord proximal de la bague 467B-11) était composé à l'origine de neuf éléments distincts dont huit étaient encore en place. Le dernier, l'ourlet bordant la crête à l'origine, n'a pas été retrouvé.

Deux tôles forment la partie courbe du tube attenante au pavillon. Elles sont maintenues ensemble par deux bagues de raccords (467B-11 et 467B-3) situées respectivement à la base du cou et par trois anneaux, deux d'entre eux (467B-10 et 467B-1/2) couvrant au sommet et les bagues mentionnées cidessus et le troisième se situant en position intermédiaire (467B-7). Les derniers éléments appartiennent à la crête. Celle-ci a été fortement abîmée, elle est en partie repliée contre le tube, dans la partie inférieure du cou. Elle fonctionnait avec l'anneau 467B-1/2 et le petit élément 467B-15 déjà cité et situé sur le pavillon.

\section{Les tubes}

Le cou est formé de deux tôles en forme de demicônes (au diamètre s'évasant progressivement de $38,8 \mathrm{~mm}$, à la base, à $70,5 \mathrm{~mm}$ près du pavillon), courbés pour former le coude au présent de l'instrument. En partie supérieure, elles s'encastrent entre les demi-faces du pavillon (fig. 39). De la même façon, elles s'emboîtent à leur base dans le tube inférieur rectiligne 467B-16. Il est probable cependant que ceci résulte d'un enfoncement postérieur de l'un dans l'autre. On pourrait imaginer que les tubes ont été réemboîtés a posteriori en force et délibérément, pour conserver à l'objet sa morphologie générale, même s'il n'était plus utilisé comme instrument de musique.

Les tôles du cou, assemblées bord à bord du côté antérieur, sont dotées d'un petit repli large de 6 à 8 $\mathrm{mm}$, sur les faces supérieure et postérieure. C'est entre ces replis que la crinière prend place.

En partie médiane, en dessous de la courbure, le martelage a créé un relief donnant l'illusion de la présence d'une bague de raccord (fig. 17). Cette fausse bague est soulignée de part et d'autre de deux traits parallèles gravés distants de $2 \mathrm{~mm}$. Au-dessus de ce décor, sous le cou, la tôle du côté droit (467B5/8) recouvre légèrement celle de gauche (467B-

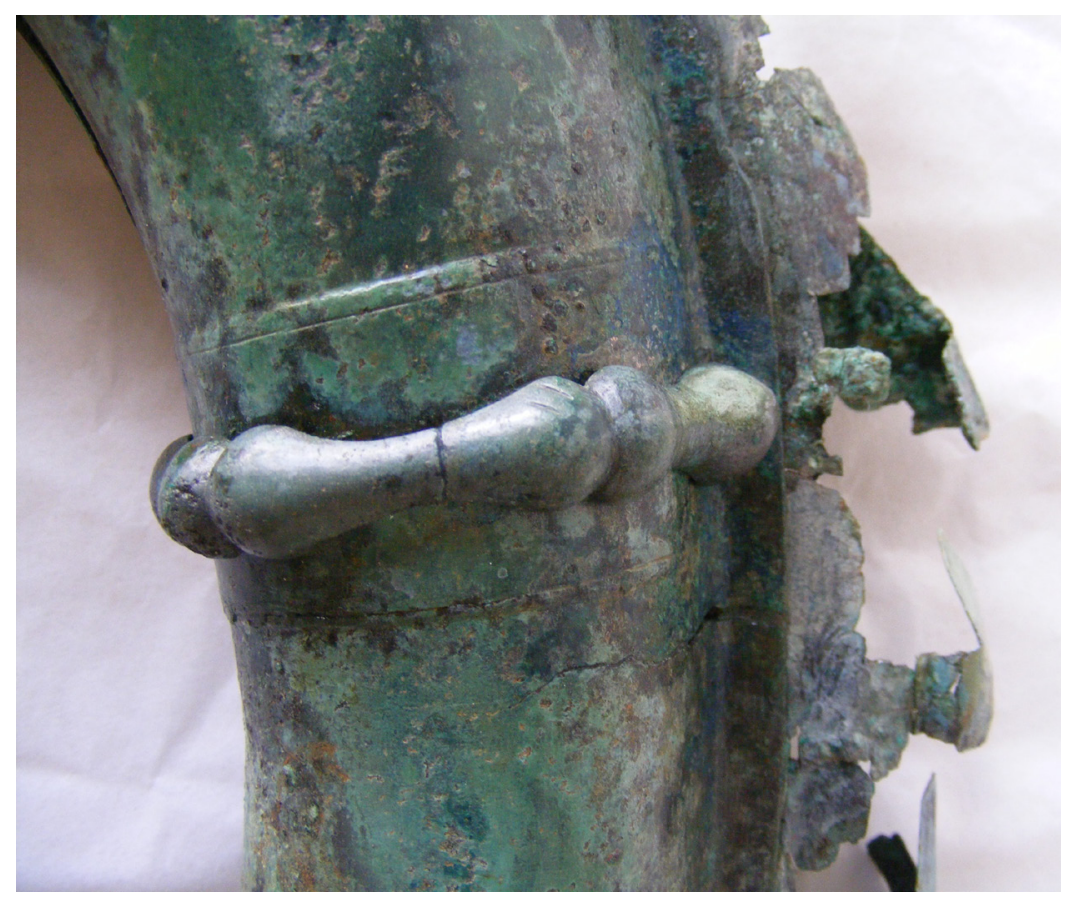

Fig. 17. La fausse bague et l'anneau moulé 467B-7 en partie médiane du cou (cl. Chr. Maniquet, INRAP).

6/9) ; en dessous, c'est l'inverse qui se produit. Les deux tôles sont jointives à la base du cou.

En ce qui concerne les dégradations, on notera de légères déformations ponctuelles du repli maintenant la crête, une petite fissure dans la partie supérieure de la tôle droite $467 \mathrm{~B}-5 / 8$ et une autre au niveau de la fausse bague. Un coup est visible en partie basse de la tôle gauche, entre les deux anneaux inférieurs.

Sur la tôle du côté gauche, la patine du métal change de couleur brusquement au passage d'une limite linéaire traversant transversalement le tube entre les deux anneaux supérieurs (fig. 18). Cette trace n'est pas liée à la proximité d'un autre élément métallique dans le dépôt car il s'agit de la face qui reposait directement sur le fond de la fosse. Sa raison d'être demeure à ce jour inexpliquée. On peut cependant imaginer qu'elle résulte de la présence contre l'objet de matière organique disparue, telle qu'une sangle qui aurait pu servir à suspendre l'instrument. Au-dessus de cette empreinte, la crête disparaît complètement, ce qui laisse imaginer que l'élément disparu a entraîné sa destruction partielle. 


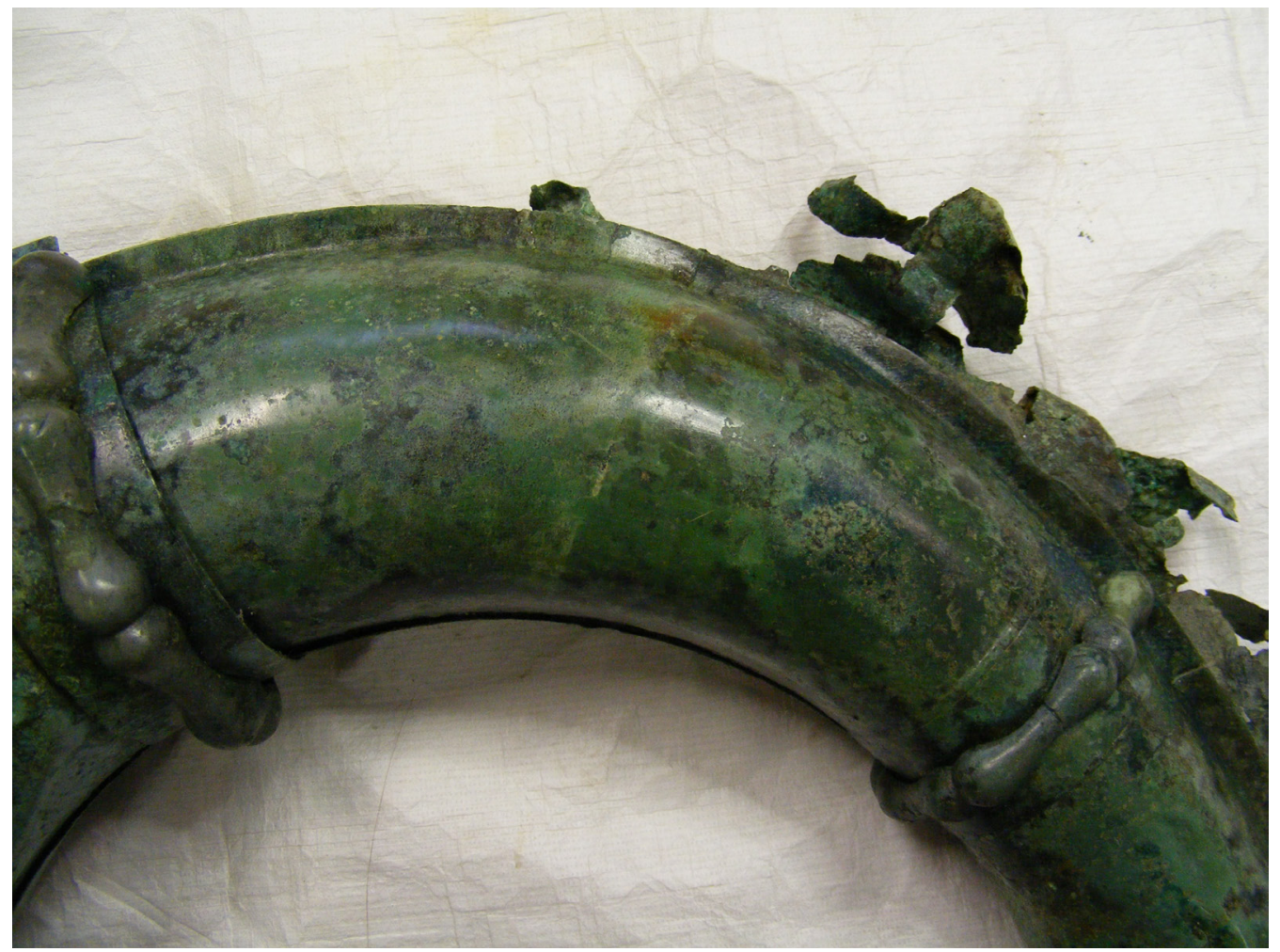

Fig. 18. Trace linéaire plus claire visible due la tôle gauche en partie supérieure du cou (cl. Chr. Maniquet, INRAP).

Les bagues de raccord

Deux bagues cachent les jonctions entre les tôles du cou et celles du pavillon (467B-11) d'une part, et celles entre le cou et la partie rectiligne du tube à la base (467B-3) d'autre part. Elles sont constituées d'une fine tôle enroulée sur elle-même mais ouverte sur l'arrière pour laisser le passage du repli des tôles du cou. La bague 467B-11, aujourd'hui légèrement écrasée, était à l'origine tronconique. La bague 467B-3, également écrasée et incomplète, porte un coup en partie supérieure droite et a légèrement pivoté vers la gauche. À l'arrière, près de son contact avec la crête, deux échancrures triangulaires sont découpées de part et d'autre de son ouverture (fig. 19). La fonction de ces encoches et d'une troisième à la base de la crête reste pour le moment inconnue.

\section{Les anneaux}

Trois anneaux enserrent les tôles constituant le cou de l'animal. Les deux premiers sont ouverts en partie supérieure ou postérieure pour le passage du repli des tôles du cou et la crête. Ils étaient décorés de six éléments en forme de maillons en huit pleins, jointifs et alignés.

Sur l'anneau situé à la jonction avec le pavillon (467B-10) (fig. 20), côté gauche, le deuxième élément de décor en partant de l'ouverture, plus court que les autres, est marqué d'une petite croix (fig. 30a). La partie supérieure de cet anneau a légèrement basculé vers l'arrière, peut-être suite au coup porté au niveau de l'oreille.

Le deuxième anneau moulé (467B-7), situé sur la fausse bague en partie médiane du cou (fig. 17), était 


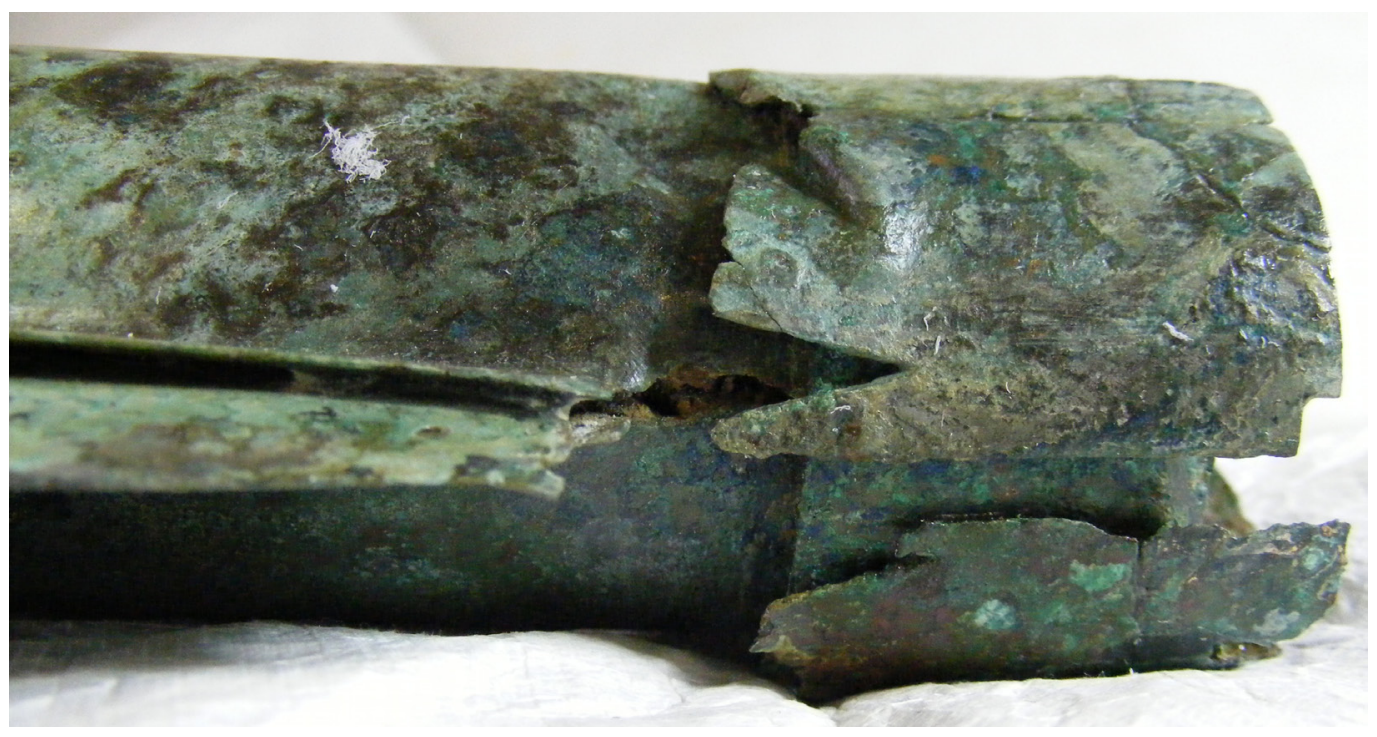

Fig. 19. Encoches triangulaires en face postérieure de la bague 467B-3 (cl. Chr. Maniquet, INRAP).

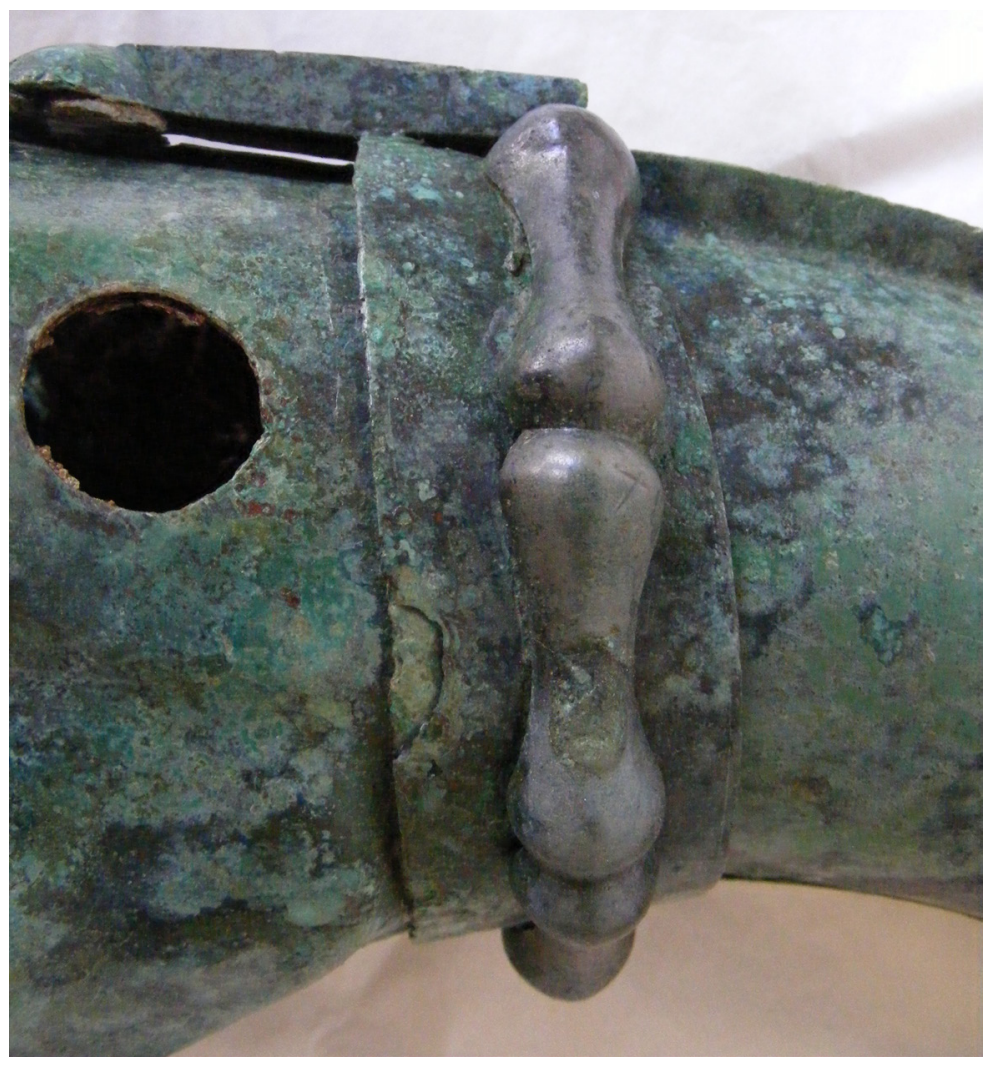

Fig. 20. Collier 467B-10 entre le pavillon et le cou du carnyx (cl. Chr. Maniquet, INRAP). 
brisé de chaque côté au milieu du deuxième maillon. Ces éléments sont plus courts que sur l'anneau précédent. Les deuxièmes en partant de l'ouverture, de part et d'autre, sont les plus longs. Côté gauche, l'un de ces maillons comporte deux petits traits parallèles (fig. 30b).

Enfin, le troisième anneau (467B-1/2) du cou recouvre la bague 467B-3 qui assure la jonction avec le reste du corps de l'instrument (fig. 21 et 22). Il est attenant à l'arrière à un élément avec lequel il a été coulé, de forme triangulaire, qui recevait la base de la crête. Les deux parties étaient brisées et séparées spatialement dans le dépôt, d'où un enregistrement initial en 467B-1 et 467B-2. Alors que l'anneau était resté à sa position initiale, l'autre partie a été retrouvée contre le tube du cou de l'instrument mais beaucoup plus haut, à mi-distance entre l'anneau qui nous concerne et l'anneau médian. La partie de cette pièce située initialement du côté droit avait disparu. Deux éléments et demi en forme de maillon en huit subsistent encore au niveau de l'anneau, tandis que la moitié d'un autre est encore attenante à l'élément postérieur.

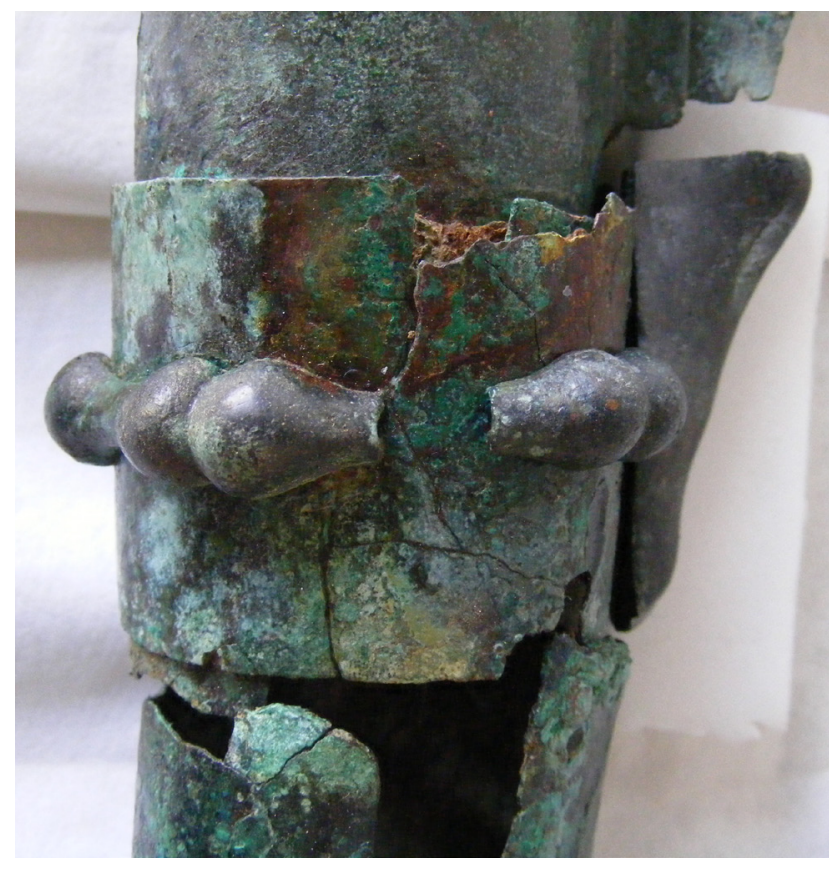

Fig. 21. Anneau 467B-1/2 en face latérale (cl. Chr. Maniquet, INRAP).

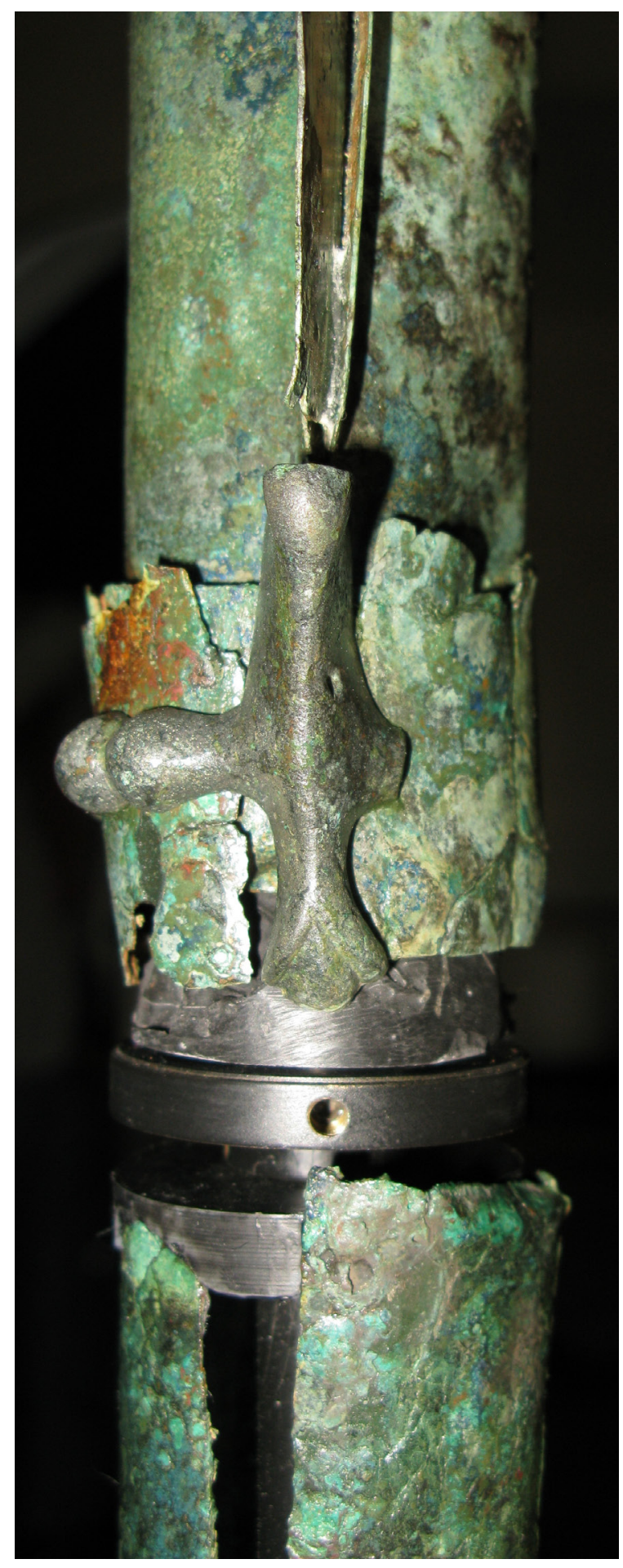

Fig. 22. L'anneau 467B-1/2 en face postérieure, à la base de la crête (cl. Chr. Maniquet, INRAP). 
Lélément 467B-1 comporte à la base un petit décor de palmette ou de bourgeon (fig. 22). La crête venait visiblement s'emboîter dans sa partie supérieure.

Un autre élément identique, brisé de la même façon, a été découvert dans la couche scellant le dépôt (Iso 2067-B2). Il appartient sans aucun doute à l'une des autres trompettes du dépôt $t^{14}$.

\section{La crête}

Plusieurs enseignes gauloises connues à ce jour, en forme de sanglier, sont pourvues d'une tôle plane, ajourée ou décorée, représentant une crête dorsale ${ }^{15}$.

La crête du carnyx de Tintignac est très abîmée mais, par chance, encore partiellement en place. Elle est coincée dans la courbure externe du cou de l'animal, entre les replis des deux tôles qui le constituent. La partie inférieure de la crête a gardé sa forme originelle. Au-dessus, la tôle plane découpée d'ajours est complètement repliée sur elle-même. Elle disparaît totalement à une dizaine de centimètres au-dessus de l'anneau médian du cou. On n'en retrouve qu'un fragment entre les lamelles de la pièce 467B-15.

Cette tôle devait, à l'instar des autres fragments de crinière découverts dans le dépôt, être doublée d'un ourlet constitué d'une petite tôle arrondie sur sa bordure périphérique, assurant ainsi un rôle de renfort et de raidisseur. Tôle et ourlet s'inséraient dans la petite pièce indépendante 467B-15 située sur
14- Un objet similaire associé à un anneau moulé (Barral 2009, 193, fig. 8/5), a été trouvé au XIX ${ }^{\mathrm{e}}$ s. sur le sanctuaire de Mandeure (Doubs). De dimensions proches de celles du fragment de Tintignac, il est également brisé. Il est généralement dessiné à l'envers de sa position originale sur l'instrument. On notera que l'anneau est constitué d'éléments jointifs réniformes et non pas en forme de maillon en huit. Il était associé à un autre anneau brisé, doté d'un décor similaire (Barral 2009, 193, fig. 8/6), qui faisait certainement partie du même instrument.

15- Chez le sanglier, la crinière est la bande de grandes soies (pouvant atteindre une quinzaine de centimètres), sur le haut du dos, qui se développe après 3 ans (Durantel 2007, 16). Ce sont des poils érectiles, dressés en cas de colère, de peur, de douleur ou pendant le rut. L'une des manœuvres d'intimidation utilisées par le sanglier sur le point d'attaquer, consiste à hérisser la crinière et dresser les oreilles, tout en poussant de sourds grognements. la tête et, à la base, dans l'appendice postérieur de l'anneau 467B-1/2. Malheureusement cet ourlet avait totalement disparu, ce qui peut s'expliquer par les coups portés sur la crinière, mais on peut également envisager qu'il n'y en a pas eu du tout.

À Tintignac, les ajours sont totalement différents d'une crête à l'autre, donc d'un instrument à l'autre. C'est l'élément principal, avec la taille des oreilles peut-être, qui permettait de les distinguer. Sur le carnyx qui nous concerne, il faudrait pouvoir déplier la tôle pour en comprendre le décor. Seule la partie basse, moins abîmée a pu être examinée (fig. 23). Au-dessus d'une goutte et de trois triangles découpés dans la tôle, des ajours différents plus élaborés pourraient laisser voir dans les pleins la forme d'un animal doté de cornes recourbées vers l'arrière de type bouc, bélier ou mouflon. Au-delà, le décor est indéchiffrable. Il serait pourtant intéressant d'approfondir l'examen afin de vérifier si cette interpré-

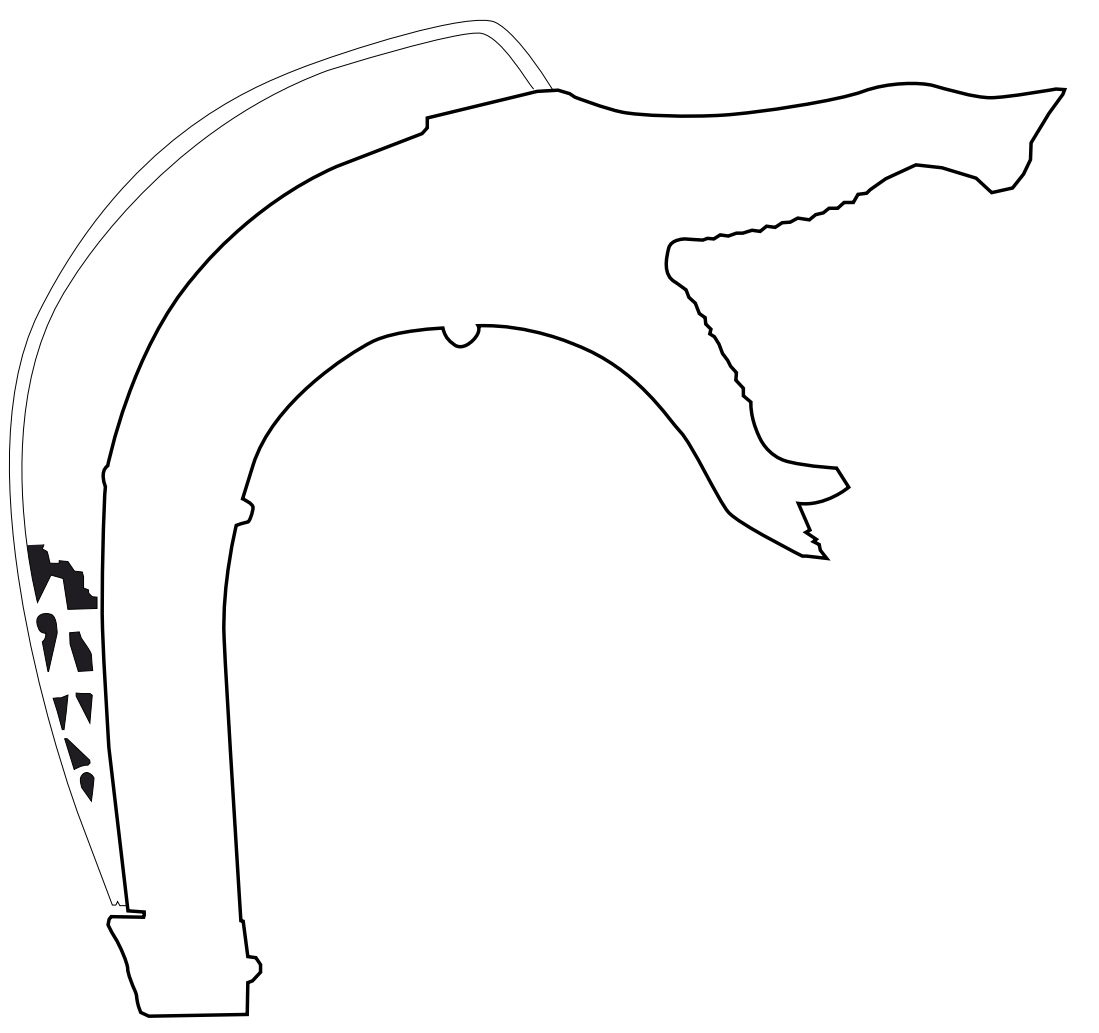

Fig. 23. La crête du carnyx avec la partie du décor identifiable (dessin Chr. Maniquet, INRAP). 


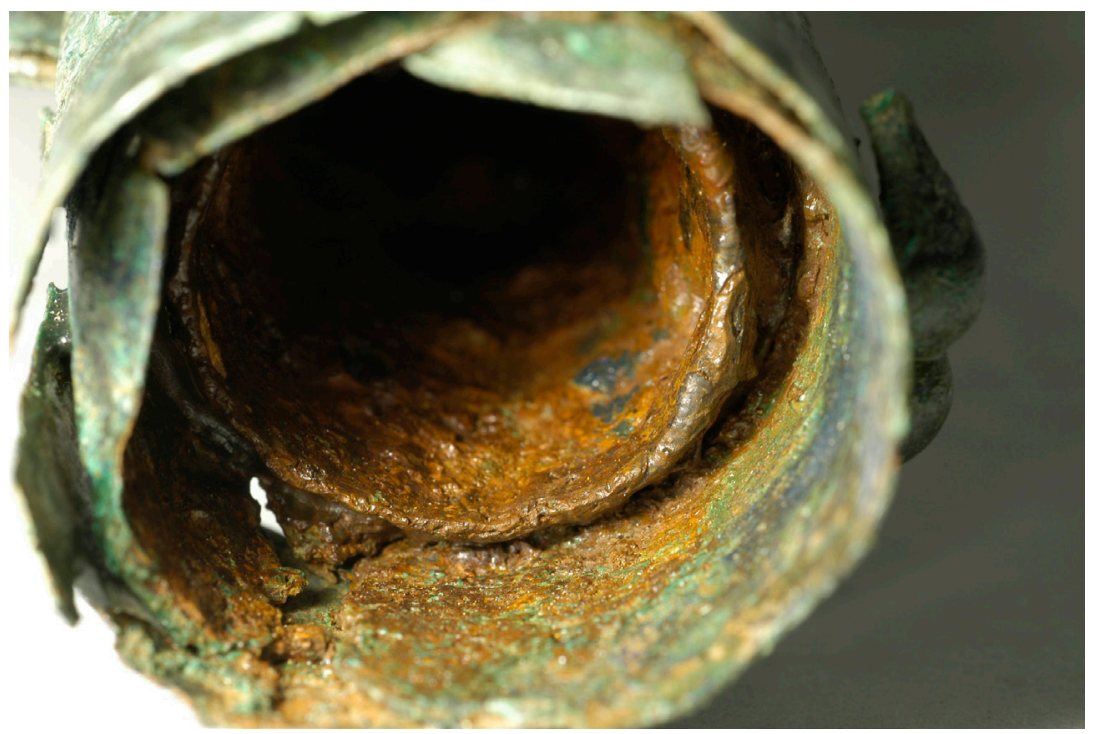

Fig. 24. Le tube 467B-17 coincé entre les tôles du cou du carnyx (cl. B. Armbruster, CNRS).

tation se vérifie et si, dans la suite du décor, d'autres animaux étaient représentés. On connaît en effet l'importante iconographie celtique des animaux affrontés (on retiendra en particulier la symbolique des dragons ou griffons sur les fourreaux d'épée laténiens) ou bien associés à l'arbre de vie ou au maître des animaux.

\section{B-17}

À l'intérieur du tube 467B-16, à l'emplacement de la bague 467B-3 et de l'anneau 467B-1/2, un autre élément (467B-17) est visible (fig. 24). Malgré plusieurs tentatives, cette pièce, collée par la corrosion, n'a pu être extraite. Son diamètre avoisine $30 \mathrm{~mm}$. Visible à la radiographie, elle semble longue de $93 \mathrm{~mm}$, de forme tronconique, et terminée en partie supérieure (dont le diamètre avoisine $20 \mathrm{~mm}$ ) par une sorte de bourrelet périphérique. Très vite, elle a été interprétée comme une embouchure qui aurait été lâchée dans la gueule du carnyx avant son enfouissement, et qui se serait coincée dans le tube. Les observations ont déterminé que cette pseudoembouchure présente des produits de corrosion du cuivre mais également du fer.
La partie basse du corps de l'instrument

Le carnyx était, dans le dépôt, brisé en grands fragments. Ainsi, le pavillon et le cou de l'animal constituaient une portion cohérente (467B), non brisée de l'instrument (fig. 6). Elle était attenante à une autre portion enregistrée en 467A, située dans le prolongement de la première, composée du tube 467A-3 et du départ du tube 467A-4, de la bague 467A-1 et de l'anneau 467A-2. Les deux portions étaient désunies par une cassure au-dessous de la bague 467B-3.

Une autre portion (457) de l'instrument reposait perpendiculairement, à plat, sur la première (fig. 4). Rien ne permettait a priori de certifier que ces éléments fonctionnaient bien ensemble. Cependant, on a pu noter des différences de morphologie des bagues en alliage moulé d'un instrument à l'autre. Or la dernière bague à la base de la portion 467 est identique à celles de la portion 457. Cet instrument semble avoir été brisé en deux grands fragments juste avant son dépôt. Celui comportant le pavillon a été soigneusement déposé au centre et au fond de la fosse. Le second morceau (457) a été déposé juste après, parallèlement à la bordure méridionale du creusement. 
Les tubes

Les tubes du corps de l'instrument, à la différence de la partie supérieure, sont constitués d'une seule tôle enroulée sur elle-même, se refermant sur la face postérieure de l'instrument.

\section{- 467A-3/467B-16}

Cette portion de tube, entre les bagues 467B-3 et 467A-1 mesure $275 \mathrm{~mm}$ de longueur. Le tube 467A-3 colle avec le fragment de tube 467B-16. Relativement intègre sur le terrain, il s'est brisé en plusieurs fragments le long de fractures existantes lors de son nettoyage. Il est légèrement écrasé en partie inférieure, à l'emplacement où il supportait le tube 457 dans le dépôt. À sa base, la bague 467A-1 et l'anneau 467A-2 couvrent sa jonction avec le tube 467A-4/474

\section{- 467A-4/474/457-6}

Le fragment de tube 467A-4 était en connexion avec la portion d'instrument 467A. Le petit fragment 474 en était, quant à lui désolidarisé et a été retrouvé plus tard à l'intérieur du casque 424 . 467A-4 et 474 mesurent à eux deux seulement $62 \mathrm{~mm}$ en dessous de la bague 467A-1. Il est impossible d'affirmer de façon catégorique que ces éléments et l'extrémité supérieure (tube 457-6) de la portion retrouvée perpendiculairement ont bien fait partie du même tube, d'autant plus que le tout petit fragment de tube 457-6 (conservé presque uniquement à l'intérieur de la bague 457-4) avait fait l'objet d'un écrasement volontaire à son extrémité.

\section{-457-5}

Le tube 457-5 est conservé sur toute sa longueur, de la bague 457-4 à la bague 457-1. Il est complètement écrasé en partie médiane, à l'endroit où il reposait sur 467A-3. D'autres petits enfoncements de la tôle sont en outre visibles sur la face postérieure, en partie haute.

\section{- 457-7/280}

La portion de tube 457-7 était brisée à la base et fracturée transversalement juste sous la bague 457-1. Un autre fragment de tube (280), découvert dans l'excroissance semi-circulaire de la fosse a trouvé des points de collage avec la base du tube 457-7. Ce fragment 280 était écrasé à chacune de ses extrémités et n'était associé à aucune bague ni anneau.
La longueur totale de la portion de tube comprise entre la bague 457-2 et l'embouchure avoisine $315 \mathrm{~mm}$, soit une longueur sensiblement équivalente à celle de 457-5. Le tube 467A-3/467B-16 mesure quant à lui $275 \mathrm{~mm}$ de longueur. On peut dès lors en déduire que la partie manquante entre les portions 474 et $457-6$ doit mesurer entre 210 et $250 \mathrm{~mm}$ de longueur.

\section{Les bagues et les anneaux}

À l'intérieur des bagues, les portions de tube sont jointes bord à bord. Ces bagues, comme celles situées au-dessus, sont constituées d'une simple tôle enroulée sur elle-même mais dont l'ouverture est systématiquement placée à l'opposé de celle du tube, c'est-à-dire en face antérieure.

Les anneaux de cette portion de l'instrument sont, contrairement à ceux situés plus près du pavillon, complètement fermés et ne sont pas ornés. Massifs et de section triangulaire, ils renforcent les jonctions tube-tube et tubes-bague (fig. 25 à 27).

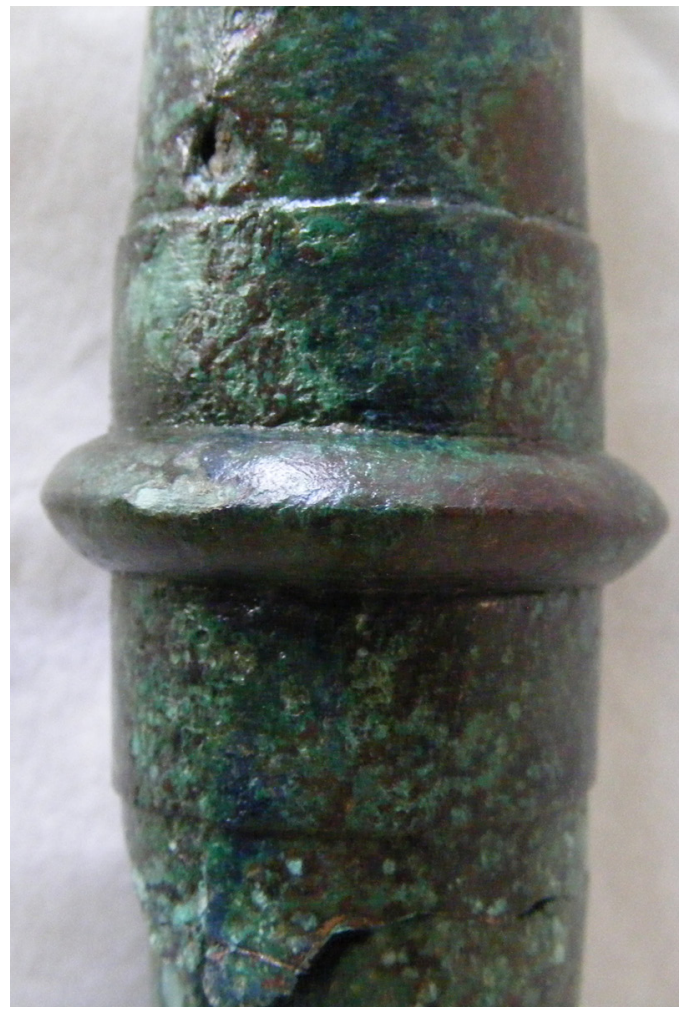

Fig. 25. L'anneau 467A-2 (cl. Chr. Maniquet, INRAP). 


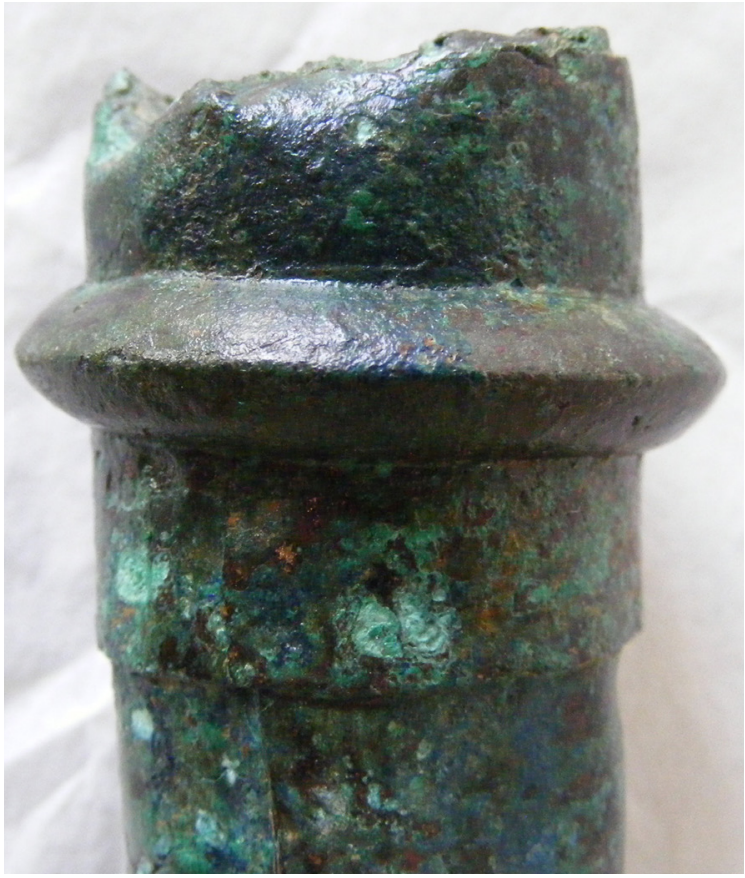

Fig. 26. L'anneau 457-3 (cl. Chr. Maniquet, INRAP).

Fig. 27. L'anneau 457-2 (cl. Chr. Maniquet, INRAP).

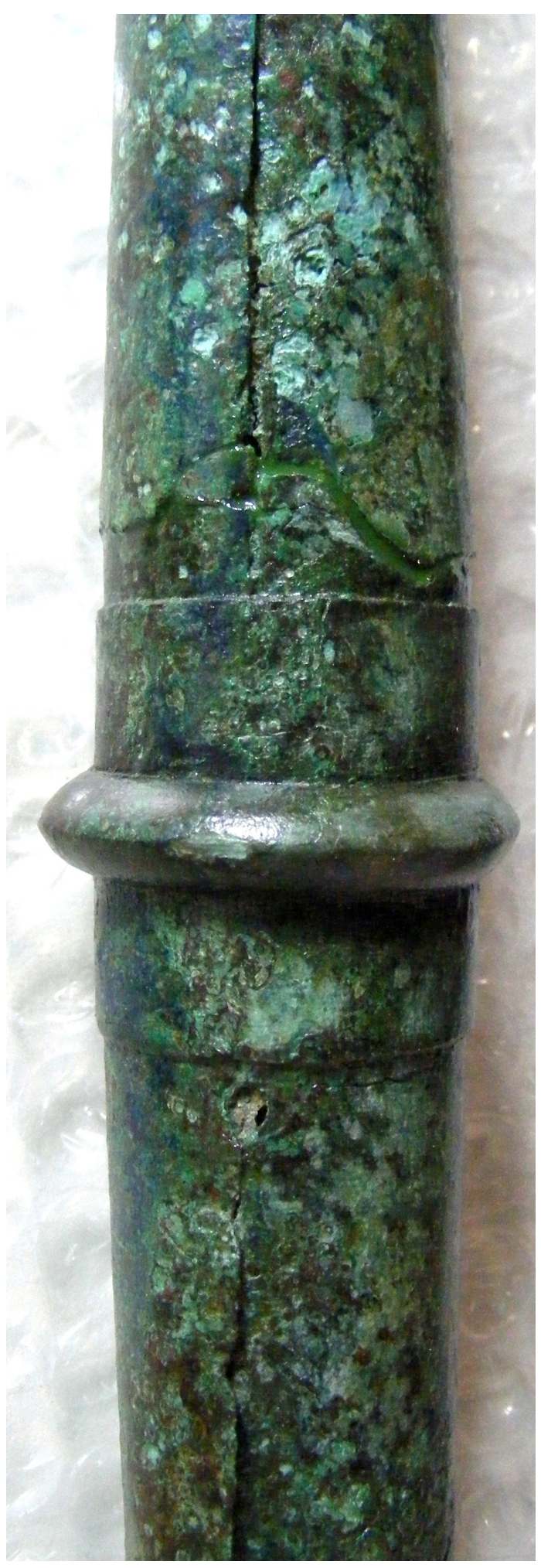




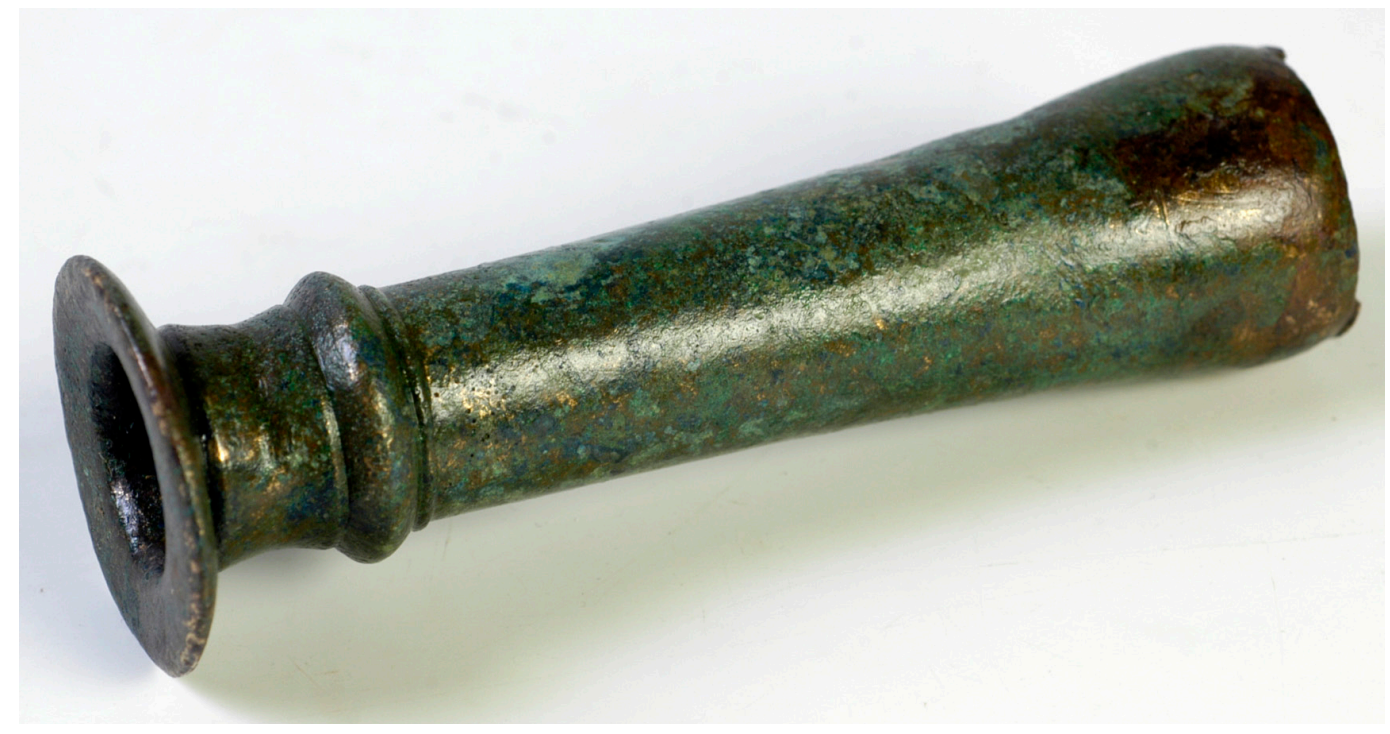

Fig. 28. L'embouchure 309 (cl. B. Armbruster, CNRS).

\section{L'embouchure}

L'embouchure du carnyx est composée d'une pièce en alliage cuivreux, au profil externe mouluré (fig. 28). Le diamètre externe du bord atteint $35,5 \mathrm{~mm}$, et le diamètre intérieur $17 \mathrm{~mm}$, ce qui confère au bord une largeur de 9,5 $\mathrm{mm}$, proche de celui des embouchures de tuba. Ce bord est plat, légèrement incliné vers l'intérieur. À l'autre extrémité, le diamètre externe était de 25,6 mm. L'intérieur est donc totalement lisse, avec un cône de queue dont le diamètre passe de $17 \mathrm{~mm}$ à $25 \mathrm{~mm}$.

En ce qui concerne son ornementation externe, on notera au-delà du bord de section triangulaire, la présence de deux petites gorges de part et d'autre d'une partie de tube marqué d'un étranglement médian. Au-dessus, un boudin périphérique (diamètre 24,8 $\mathrm{mm}$ ) de section triangulaire au sommet arrondi rappelle les anneaux moulés situés sur le corps de l'instrument. Au-dessus, deux petits bourrelets délimitent une partie lisse de la queue qui s'évase progressivement (passant de 20,6 $\mathrm{mm}$ à 28,5 $\mathrm{mm}$ ) avant de s'étrangler à nouveau (diamètre $25,6 \mathrm{~mm}$ ). Le second bourrelet ne subsiste que ponctuellement en bordure de l'embouchure. Le premier tube du corps de l'instrument devait être emboité à l'intérieur. On a pu noter, après restauration, la présence à $9,8 \mathrm{~mm}$ de cette extrémité d'un tout petit trou qui a pu recevoir un rivet. Cette perforation pourrait laisser imaginer que l'embouchure n'était peut-être pas fixée en permanence sur l'instrument, mais qu'elle pouvait être démontée, si nécessaire. L'épaisseur du métal oscille entre 1,1 et $1,8 \mathrm{~mm}$ et atteint près de $4 \mathrm{~mm}$ au niveau du faux anneau et juste au-dessus du bord.

\section{Le poids de l'instrument}

Les différentes parties de l'instrument lui confèrent un poids total de $2 \mathrm{~kg}$ environ, poids tout à fait raisonnable pour un instrument de si grande taille. Il est en outre nettement en deçà de celui de toutes les restitutions amateures ou scientifiques réalisées jusqu'alors.

\begin{tabular}{|l|l|}
\hline Portion & Poids \\
\hline Pavillon et tube 467B & $962 \mathrm{~g}$ \\
\hline Tubes 467A + 474 & $197 \mathrm{~g}$ \\
\hline Tube 457 & $282 \mathrm{~g}$ \\
\hline Tube 280 & $55 \mathrm{~g}$ \\
\hline Embouchure 309 & $91 \mathrm{~g}$ \\
\hline Sous-total & $1587 \mathrm{~g}$ \\
\hline Oreille 464 & $175 \mathrm{~g}$ \\
\hline Poids total & $1762 \mathrm{~g}$ \\
\hline Estimation du poids (avec une deuxième oreille) & $1937 \mathrm{~g}$ \\
\hline Estimation du poids total (avec portion du tube manquante) & env. $2000 \mathrm{~g}$ \\
\hline
\end{tabular}




\section{Les aspects technologiques \\ (B. Armbruster)}

L'objectif de l'étude technologique du carnyx et du casque de Tintignac est de reconstituer les différentes techniques, la chaîne opératoire de leurs modes de fabrication et l'outillage indispensable. Ces deux objets en bronze, en bon état de conservation, ont été examinés à l'aide d'un microscope binoculaire pour saisir des traces laissées en surface par les procédés et outils employés. L'identification et l'interprétation de ces stigmates sont ainsi primordiales pour comprendre les étapes de la mise en forme, de la décoration et de l'assemblage. Ces observations ont été accompagnées par des études du laboratoire de restauration et croisées avec des informations radiographiques, des résultats d'analyses de la composition élémentaire du métal et des sections métallographiques obtenues en laboratoire d'archéométrie (par F. Adamski, M. Pernot et P. Piccardo). Ainsi, en complément de l'examen des traces d'outils, les clichés radiographiques ont rendu visibles les traces de martelage, le nombre d'éléments constitutifs des objets et les points de jonction mécaniques ou métalliques (fig. 29). De plus, ces clichés discriminent clairement les parties massives issues de la coulée des éléments en tôle. On distingue à l'étude macro- et microscopique différents stigmates, notamment des traces d'outils résultant du martelage, visibles comme de petites plages aplaties, ou du polissage, perceptibles par de très fines stries parallèles ou diffuses, puis des structures des surfaces, comme des rugosités dues à la coulée, ou encore des restes de brasure. On peut identifier ces dernières à l'intérieur de l'objet par une masse considérable d'étain apportée aux points de jonctions, aujourd'hui très altérée par la corrosion. À l'extérieur, les brasures se distinguent de la surface bien polie du bronze par une couleur grisâtre. Des points d'assemblage renseignent sur les choix techniques de l'artisan pour joindre plusieurs éléments bien adaptés à la forme et à la fonction de l'objet. Les observations des surfaces faites pendant et après la restauration permettent de déceler des détails de

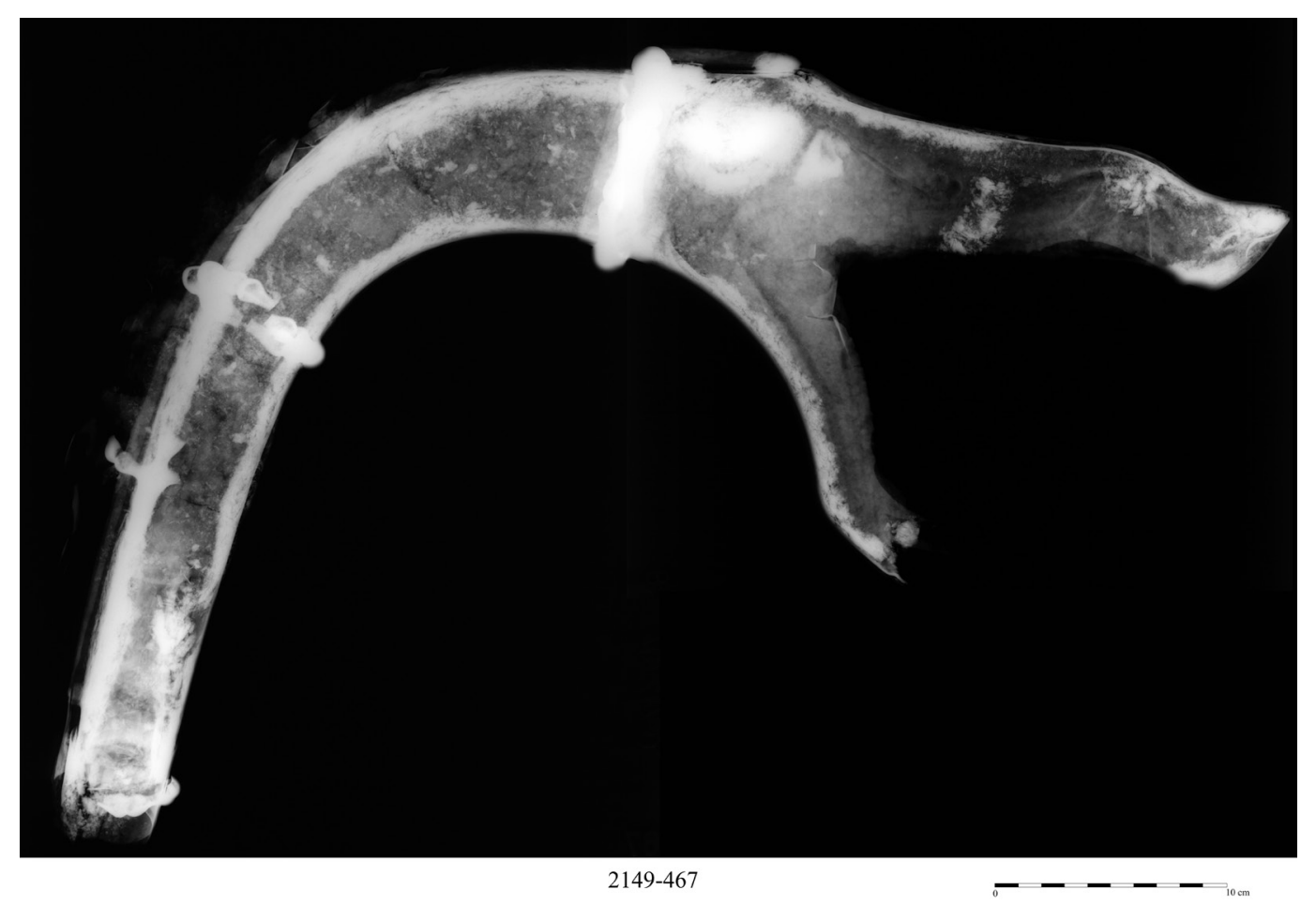

Fig. 29. Radiographie du carnyx (cl. C. Gargam et Apave-I'Union). 
la fabrication, de l'utilisation et finalement de l'altération lors de l'enfouissement des objets.

Du point de vue de la conception technique, le carnyx et le casque ont plusieurs aspects en commun. Les deux constituent des œuvres de chaudronnerie dotées d'une fonction double, à la fois conçues comme des représentations figuratives et comme instrument sonore à vent ou casque. Il s'agit dans les deux cas d'objets creux, de grande taille, de forme tridimensionnelle, majoritairement en tôle. Ils sont composés de plusieurs modules constitutifs, fabriqués séparément, puis assemblés.

En ce qui concerne le carnyx, plusieurs points doivent être pris en considération dans l'étude technique d'un objet aussi complexe. L'ensemble doit être conçu, techniquement, de manière à rester stable lors de son utilisation tout en étant le plus léger possible. Les tôles ont en conséquence de faibles épaisseurs, comprises entre 0,3 et 1,3 $\mathrm{mm}$. La plupart de ces pièces sont en tôle martelée et d'autres obtenues par fonderie. Les éléments en tôle martelée sont : la tête, composée de deux coques et du boutoir, le cou en deux coques, la crinière, l'élément 467B-15, cinq bagues de raccord cylindriques et quatre segments de tubes. Trois anneaux décorés, trois anneaux simples et l'embouchure constituent les éléments coulés.

Pendant une longue chaîne opératoire, depuis le lingot brut de coulée jusqu'à l'obtention des tôles tubulaires ou en forme de tête de sanglier, le produit initial subit une transformation entièrement par déformation plastique.

Dans la mise en forme du relief interviennent non seulement le martelage, mais également la ciselure pour les détails décoratifs ${ }^{16}$. La déformation par marteau et enclume, ou à l'aide de ciselets et d'un petit marteau sur un ciment de ciseleur, rend le métal dur et cassant. De ce fait, l'artisan recourt régulièrement à des recuits, indispensables pour rendre aux tôles en bronze la capacité de déformation nécessaire par recristallisation.

Le découpage compte également parmi les techniques de la déformation plastique. Il était exécuté sur une surface dure de frappe à l'aide d'un ciseau affûté et d'un marteau. L'artisan découpait les contours exacts de toutes les pièces en tôle, formes

16- Perrier 1979 ; Bois 1999. simples comme des rectangles pour les éléments tubulaires, ou plus complexes avec les arrondis des coques du pavillon. Les fentes des deux côtés de la gueule ont été vraisemblablement obtenues par découpage à l'aide d'un ciseau. Certaines décorations sont aussi découpées.

\section{Les anneaux et l'embouchure}

L'embouchure et les anneaux (les trois anneaux décorés dont deux ouverts et un fermé, et les trois exemplaires lisses et fermés) sont coulés selon la technique de la cire perdue. La fonderie a été choisie pour ces éléments pour des raisons fonctionnelles. Les anneaux doivent être rigides pour tenir et assurer les jointures de cerclage. Deux anneaux décorés portent des marques, l'un une croix, l'autre un double trait (fig. 30), frappées avec un ciselet plat. Ces objets creux et annulaires ont été coulés sur un
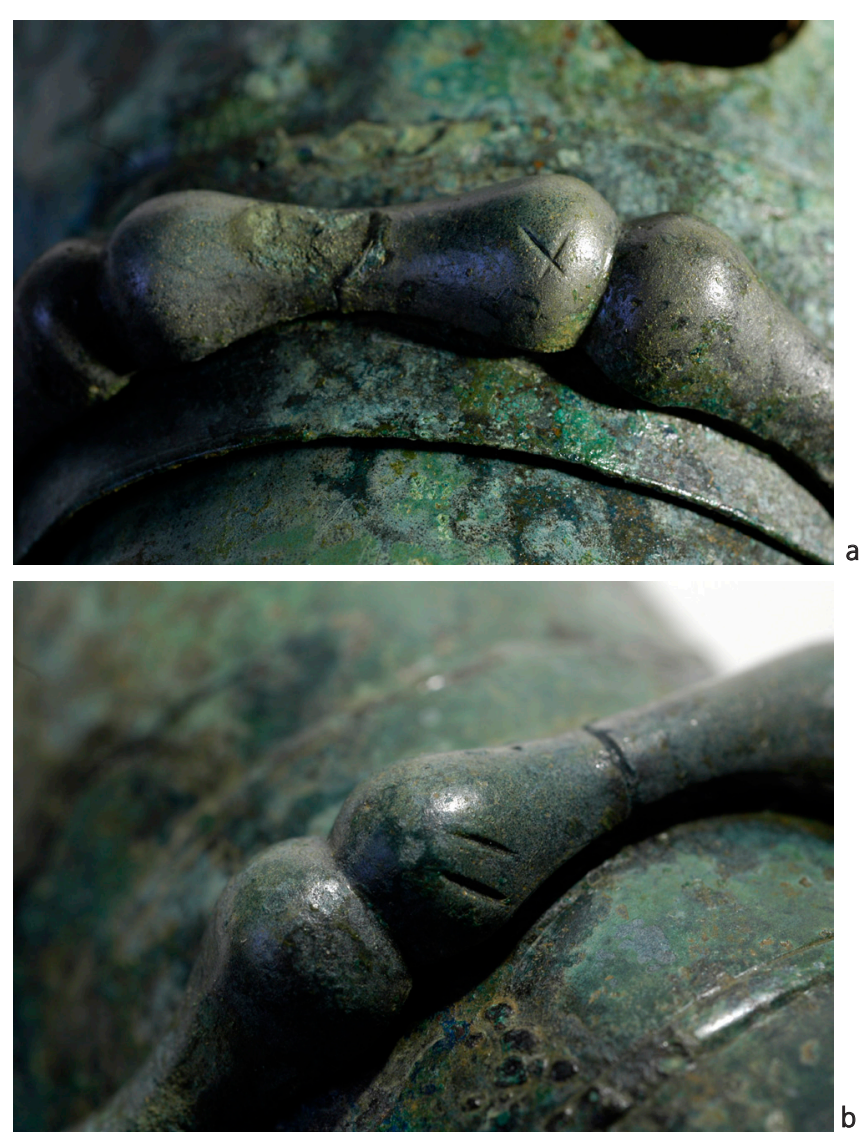

Fig. 30 a et $b$. Anneaux décorés portant des marques, I'un une croix, l'autre un double trait (cl. B. Armbruster, CNRS). 
noyau en argile. Les modèles en cire des anneaux étaient déjà légèrement creusés à l'intérieur pour minimiser le poids de chaque élément coulé puis de l'ensemble.

Malgré sa forme creuse l'embouchure est un produit de coulée lourd, l'élément le plus lourd de l'ensemble. Son décor de quelques cannelures en relief était prévu dans le modèle en cire. L'embouchure est réalisée vraisemblablement par coulée avec le but d'avoir une première pièce solide sur laquelle repose tout le poids de ce long instrument pour le maintenir avec une main, quand l'autre main assure plutôt l'orientation verticale.

Il est remarquable que des alliages avec des propriétés mécaniques distinctes ont été utilisés pour la coulée à la cire perdue des bagues et de l'embouchure, ou pour la coulée en lingotière d'ébauches destinées à la fabrication des tôles (voir infra, Étude métallurgique, de M. Pernot). Il s'agit là aussi manifestement de choix techniques de l'artisan.
Le pavillon et le cou

Pour obtenir tous les éléments du pavillon et du cou, il a fallu préparer huit tôles indépendantes ainsi que trois anneaux coulés, ouverts et décorés d'un relief. Les éléments en tôle du pavillon ont été mis en forme à l'aide de marteaux et enclumes particuliers, et ensuite décorés par ciselure pour certains. Les reliefs formant les dents, l'œil et le larmier ou encore le groin ont été ainsi réalisés à l'aide de ciselets (fig. 32). Les coques en tôle de formes et de décors inversés composant la tête et la nuque attestent une remarquable maitrise de l'art de la chaudronnerie. Des traces de martelage et de ciselure bien visibles sur la surface intérieure du pavillon du carnyx indiquent sans équivoque la fabrication par déformation plastique (fig. 33). L'artisan a poli soigneusement toute la surface extérieure, lui donnant un aspect homogène et bien lisse.

Dans la partie courbe du cou, une fausse bague a été travaillée par ciselure, comme un élément de décor sans fonction pratique (467B-7) (fig. 31). Cette imitation peut probablement être attribuée à un mo-

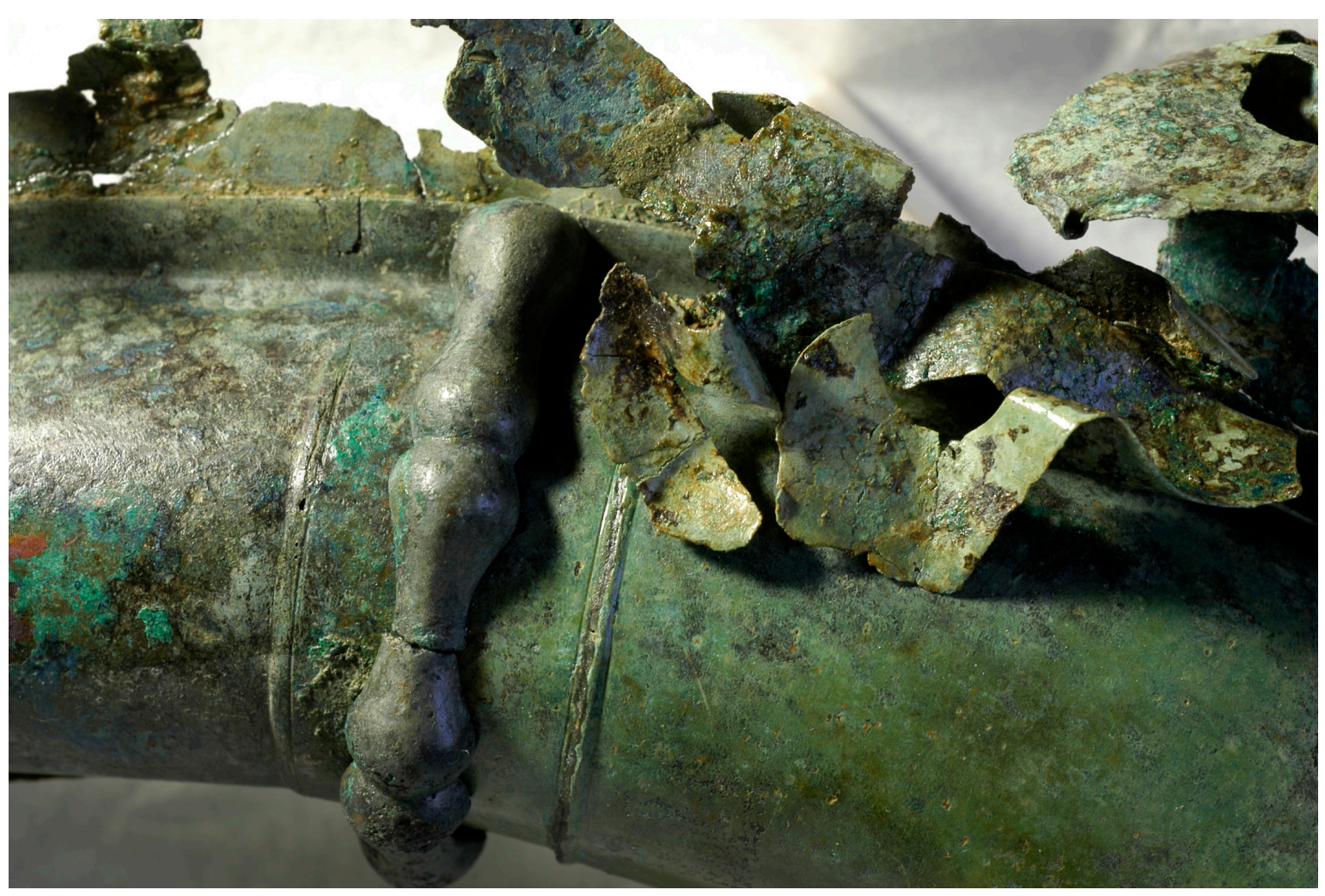

Fig. 31. Fausse bague travaillée par ciselure (cl. B. Armbruster, CNRS). 

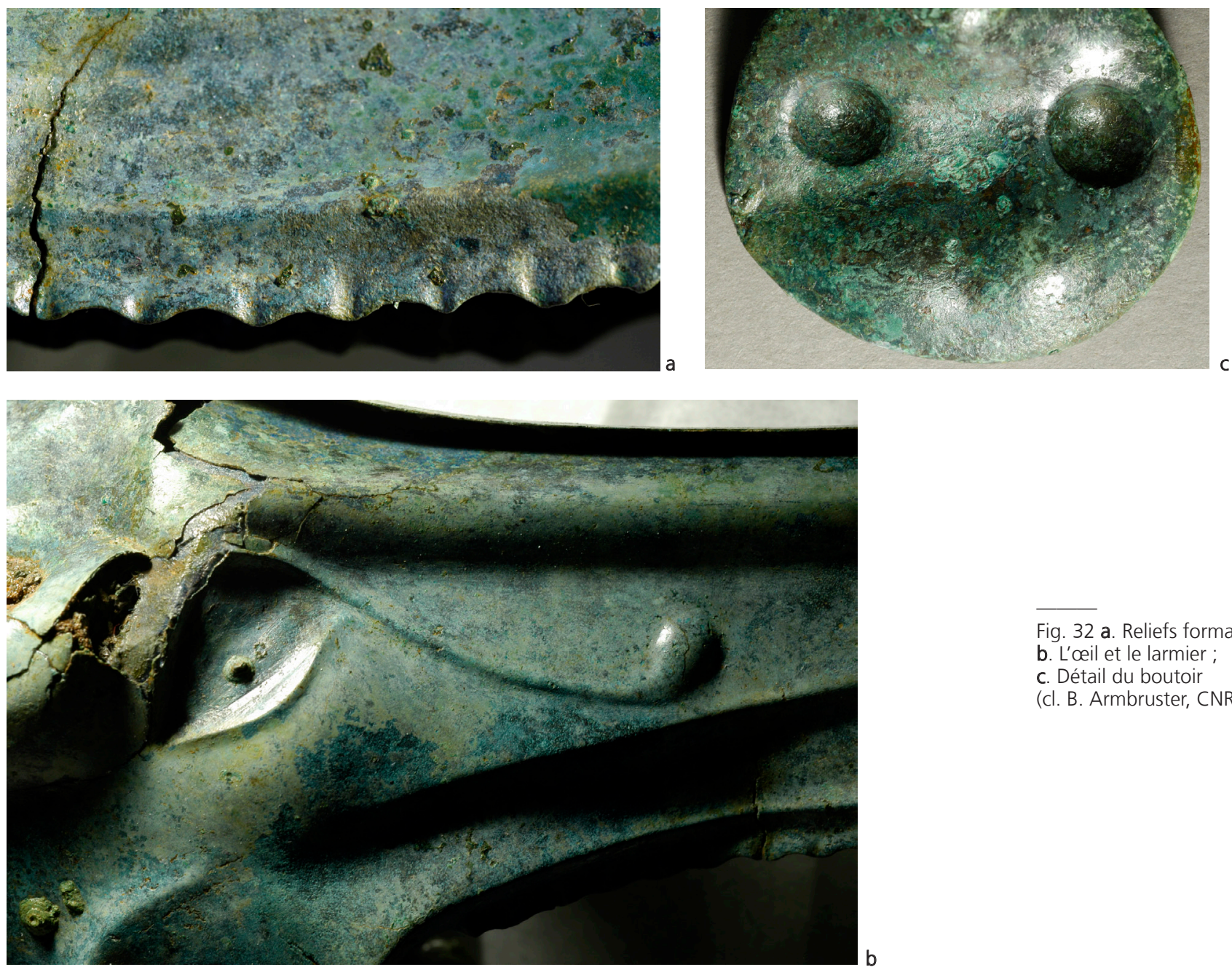

Fig. 32 a. Reliefs formant les dents ; b. L'œil et le larmier :

c. Détail du boutoi

(cl. B. Armbruster, CNRS).

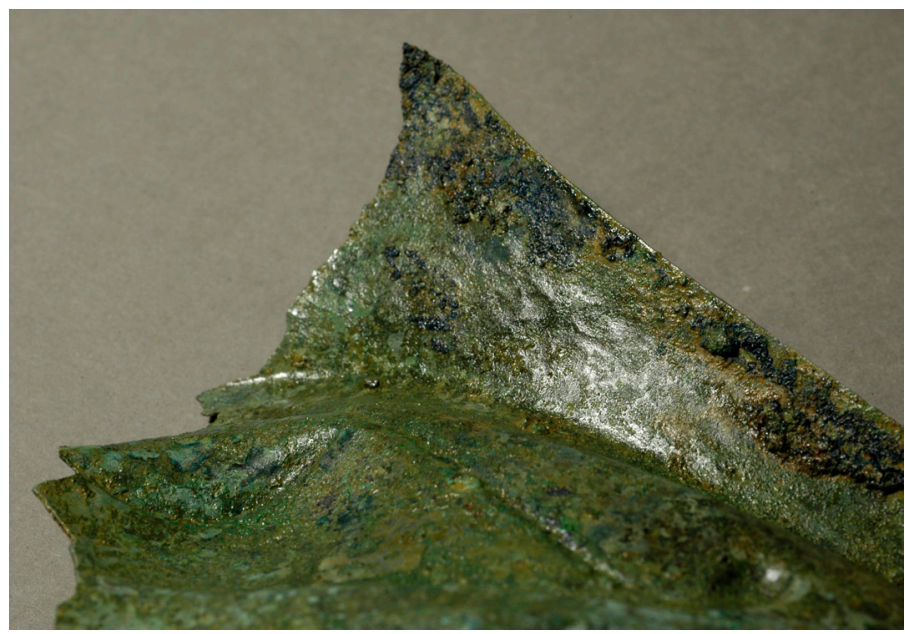

Fig. 33. Traces de martelage et de ciselure (cl. B. Armbruster, CNRS). 


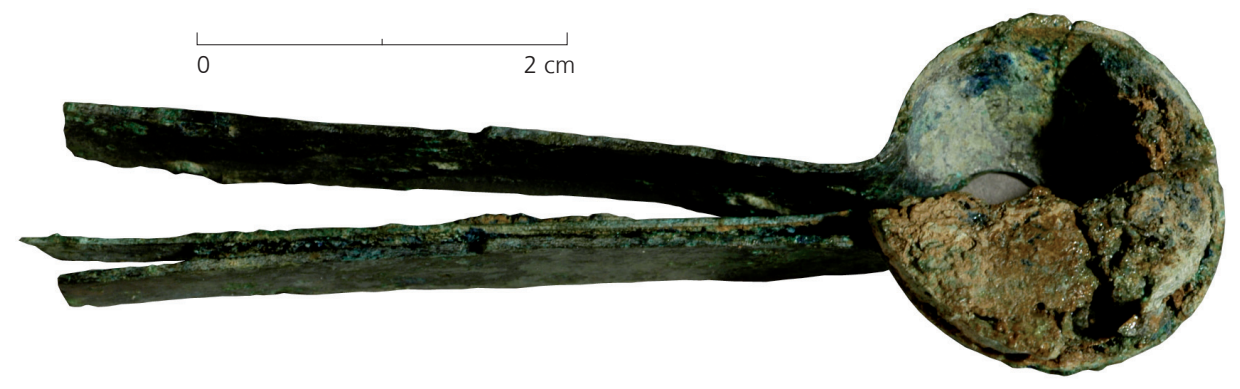

Fig. 34. Petit élément 467-B15 fixé à l'origine sur la tête du carnyx (cl. B. Armbruster, CNRS).

dèle de carnyx antérieur portant une bague réelle et fonctionnelle à cette place. Ou s'agit-il d'un choix de rythme de la position des anneaux?

La crinière a été travaillée sur une fine tôle lisse dont le décor ajouré a été découpé à l'aide d'un ciseau aiguisé. Elle était doublement fixée, par blocage et par brasure, pour assurer sa position verticale. Des restes de brasure dans l'élément 467B-15 attestent de son mode de fixation sur le sommet de la tête (fig. 34).

La forme ouverte des deux anneaux décorés, situés au niveau supérieur du pavillon, leur permet d'assurer le cerclage en combinaison avec des bagues, tout en bloquant la crinière entre les deux bords supérieurs repliés du cou.

Les yeux portaient des incrustations, formées d'un ensemble iris/pupille en lignite noir, cerné de blanc, probablement de l'étain, leur donnant un aspect polychrome (fig. 35). Dans le relief de l'œil droit dont l'incrustation est manquante, des aspérités observées servaient probablement pour la fixation de l'œil à l'aide d'un adhésif.

Les éléments tubulaires du carnyx

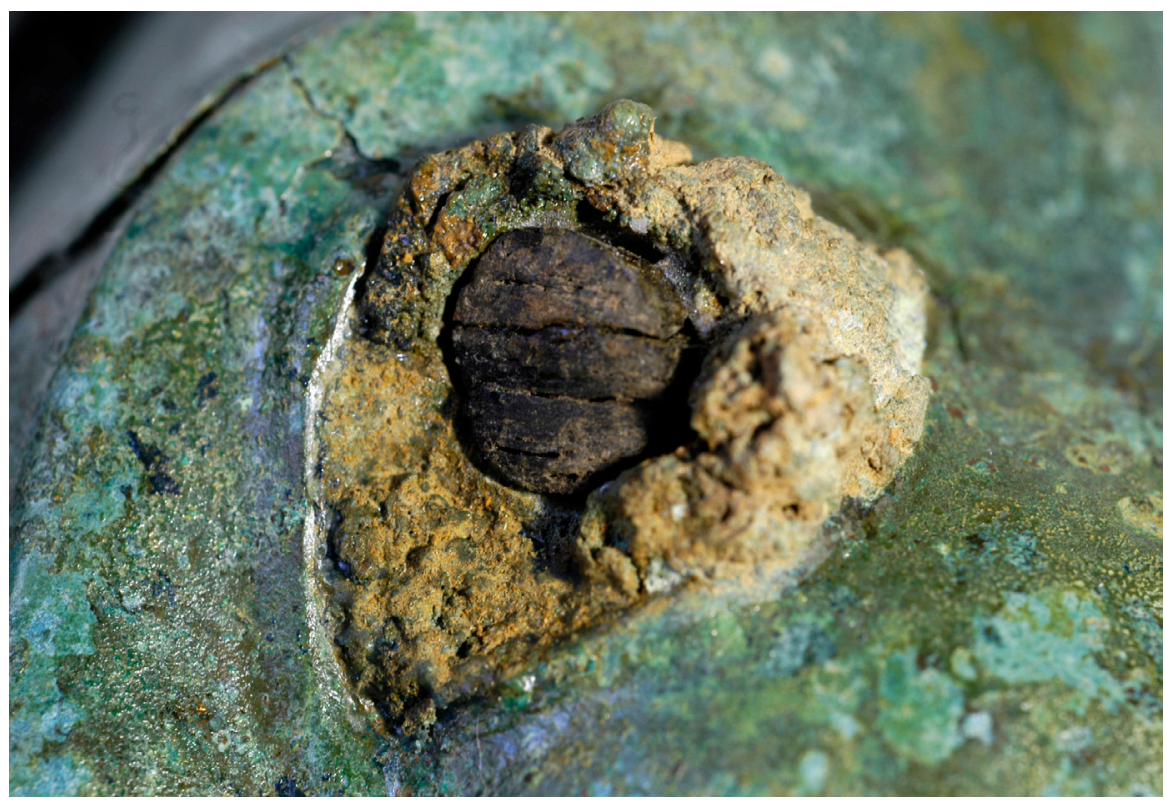

Fig. 35. Détail de l'œil gauche conservé (cl. B. Armbruster, CNRS). 
La partie tubulaire légèrement conique de la trompe est constituée de quatre éléments de tubes dont chacun est réalisé d'une tôle formant un cylindre, quatre bagues cylindriques en tôle et quatre anneaux coulés (fig. 36). La forme tubulaire a été obtenue en enroulant en longueur des tôles de contour rectangulaire ou sub-rectangulaire autour d'une âme en bois ou en métal légèrement conique. Les jonctions bord à bord sont fermées par brasure puis polies à l'extérieur.

L'embouchure, un élément issu de la coulée à la cire perdue, était fixée au dernier tube par emboîtage.

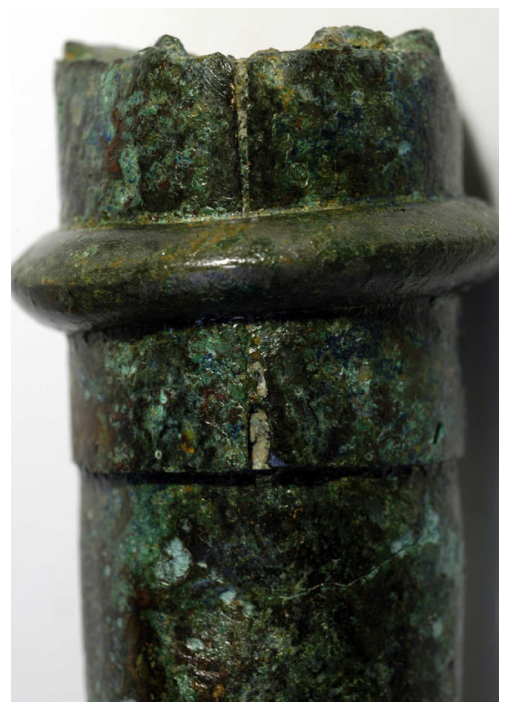

Fig. 36. Bague en tôle et anneau moulé à la jonction de deux tubes (cl. B. Armbruster, CNRS).
L'oreille

Une des deux oreilles restaurées et associées à cet instrument, est composée d'une grande tôle fine en relief et d'un petit croissant en tôle lisse. La grande tôle martelée porte une nervure courbe ciselée le long de l'axe longitudinal qui lui confère de la stabilité par le relief, et mouvement (fig. 37a). Une extrémité a été transformée en tube en utilisant un dé à emboutir à rainures et une tige de section circulaire. Cette partie cylindrique est fermée bord à bord (fig. 37b). Le croissant fixé par brasure renforce la transition entre la nervure en creux et la partie tubulaire servant de point de jonction avec la tête du sanglier. Un bourrelet de corrosion du métal utilisé pour la brasure est visible à l'intérieur du pavillon,
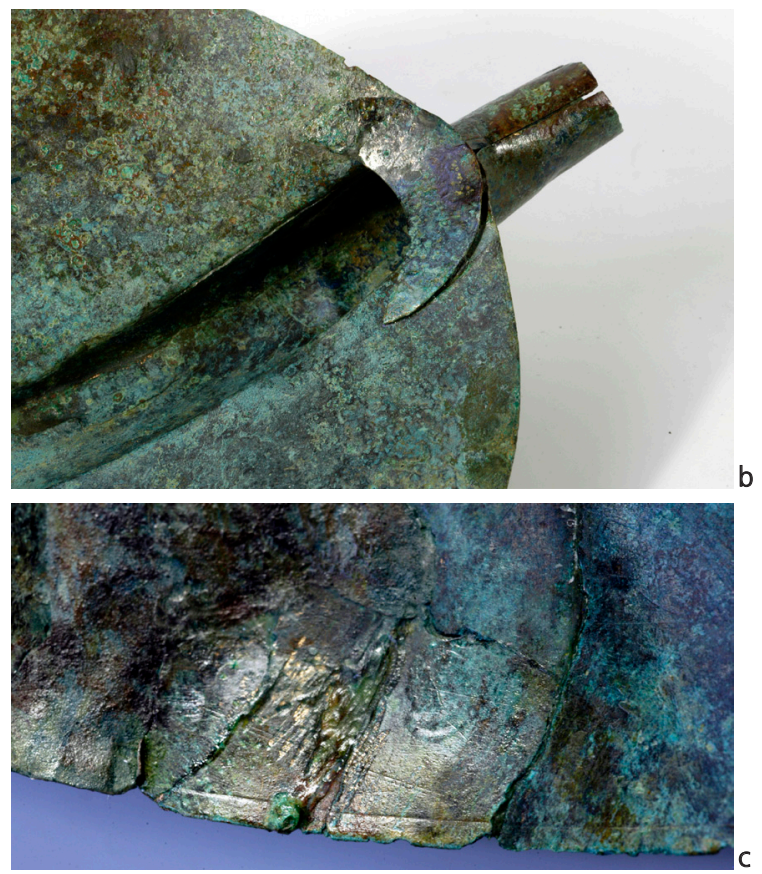

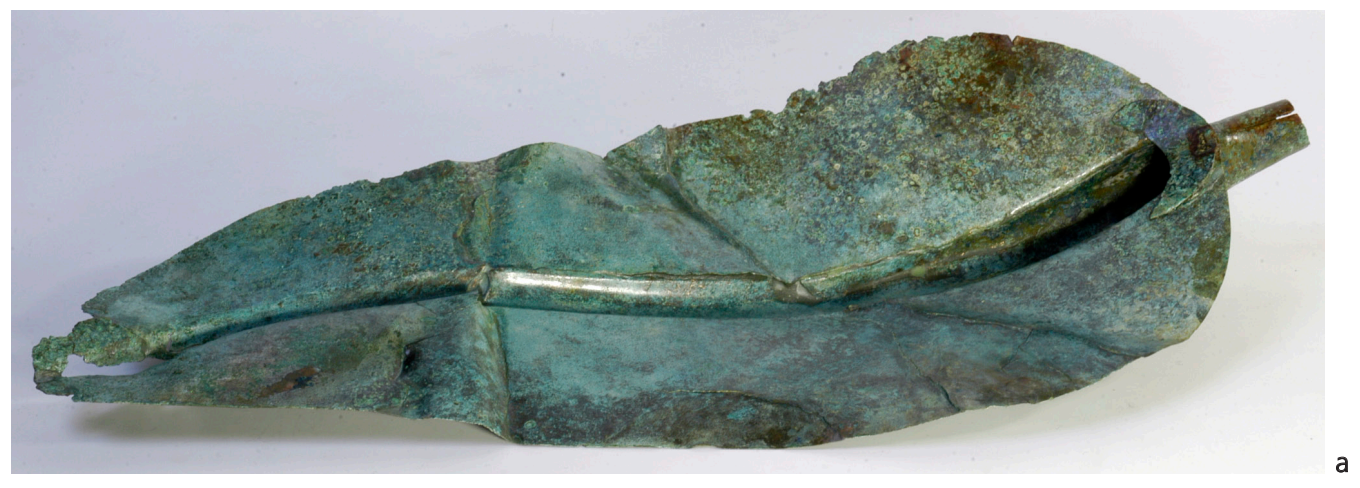

Fig. 37 a. L'oreille ; b. Détail de la douille de l'oreille ; c. Détail de la bordure de l'oreille (cl. B. Armbruster, CNRS). 


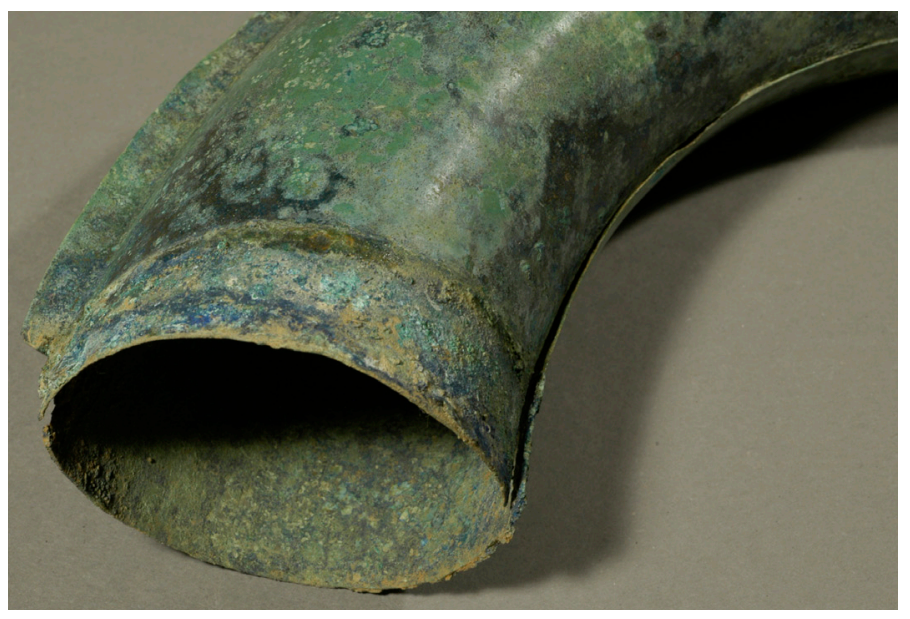

Fig. 38. Traces de brasures à l'étain (cl. B. Armbruster, CNRS).

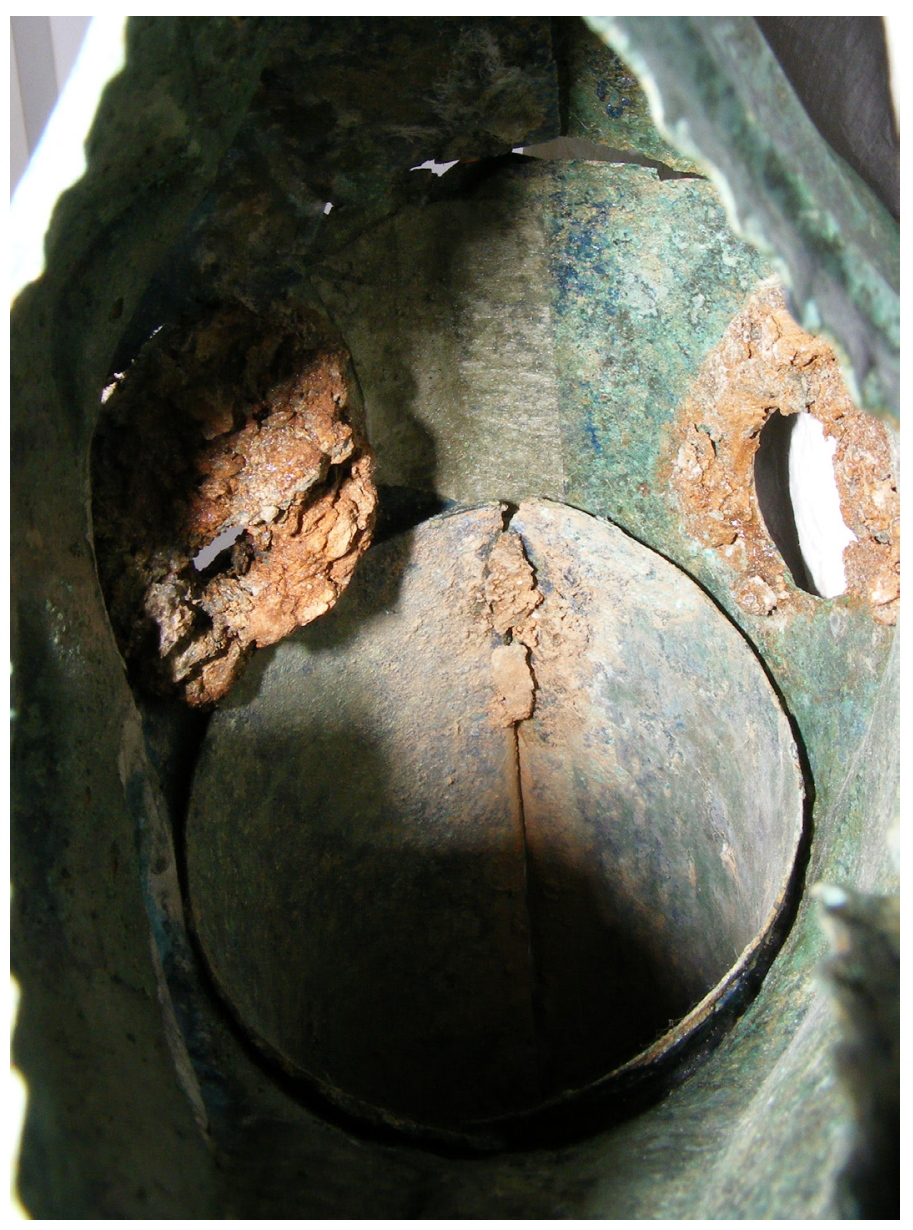

Fig. 39. Restes de brasure à l'intérieur du pavillon, au niveau des orifices prévus pour les oreilles (cl. Chr. Maniquet, INRAP). en périphérie du trou de l'oreille gauche. À droite, un amas de métal informe bouche presque totalement la cavité de l'autre oreille. Cette brasure renforçait la jonction entre les appendices auriculaires et le pavillon (fig. 39). Une fine ligne ciselée décore le bord de l'oreille (fig. 37c). Effectuée par déformation plastique elle a en même temps la fonction de stabiliser ce long bord courbe.

\section{Les procédés d'assemblage}

L'assemblage des éléments du carnyx a été effectué par raccords mécaniques à froid et par raccords métalliques à chaud. Les raccords mécaniques à froid ont été réalisés par emboîtage et cerclage à l'aide des bagues et anneaux. En revanche, les raccords à chaud ont été obtenus par brasure d'étain (fig. 38). Cette brasure est appliquée soit sous forme de petits grains, de nodules, soit de bandes, selon l'endroit, à l'intérieur du joint. Cet alliage de brasure tendre est chauffé pour atteindre sa basse température de fusion soit dans un feu de charbon de bois soit à l'aide d'un fer à souder, peut-être même à l'aide d'une flamme de lampe à l'huile et d'un chalumeau. Le fer à souder chauffé dans des braises est posé à l'endroit de la brasure pour y transmettre sa chaleur à travers la tôle en bronze, excellent conducteur de chaleur.

Par endroit, on trouve même une combinaison de modes d'assemblage, à chaud et à froid, en l'occurrence sur les oreilles, aux cinq jonctions avec les bagues et anneaux et pour la fixation de la crinière. Il s'agit d'un travail de précision avec des jointures solides et adaptées à la fonction du carnyx, permettant une circulation d'air fluide dans cet instrument à vent. Les éléments en tôle ont donc été assemblés par plusieurs modes de jonction. Selon des questions fonctionnelles, les artisans ont choisi des jonctions de tôles soit bord à bord sur la longueur soit par superposition des bords pour les tubes emboîtés.

Une fois les quatre tubes fermés achevés, ils ont été assemblés en commençant par le diamètre le plus grand. À chaque jonction, une bague puis un anneau de cerclage ont servi d'éléments d'assemblage. Les anneaux de cerclage de cette partie du carnyx ont la forme d'un cercle fermé de section en C à surface lisse. Ils sont issus de la coulée. 
Tandis que la brasure du groin et des deux coques de la tête est bord à bord, les oreilles ont été emboîtées en introduisant la partie tubulaire dans les ouvertures des coques respectives et en apportant beaucoup de matière de brasure à l'intérieur (fig. 39).

\section{Le possible étamage}

L'étude métallographique montre une fine couche d'étain à certains endroits, indiquant un possible étamage (fig. 43) qui pouvait donner un aspect argenté au carnyx. Plusieurs modes d'étamage artisanal sont connus pour la Protohistoire. On a pu tremper l'objet dans un bain d'étain liquide, ou bien appliquer l'étain sur la surface chaude en étalant l'étain liquide à l'aide d'un chiffon, ou encore étamer dans un bain électrochimique, sans apport d'électricité. Dans ce dernier cas une solution aqueuse contenant de l'étain et du tartre de vin (bicarbonate de potassium) agit dans un état proche de l'ébullition. Des recherches pour éclairer cette question d'étamage sont en cours.

\section{La chaine opératoire de la fabrication du carnyx}

La fabrication du carnyx comprend plusieurs étapes se succédant selon un ordre technique. Avant toute mise en œuvre d'un objet métallique l'artisan développe un projet qui peut exister sous forme de maquette, de dessin ou plus simplement d'une idée. D'abord fondre et allier les composants des différents alliages dans un creuset, puis couler des lingots dans une lingotière. Ils serviront comme produits préliminaires pour la fabrication des tôles. La coulée d'anneaux lisses, d'anneaux décorés en relief et de l'embouchure par le procédé à la cire perdue peut être également réalisée dans ce contexte de fonderie.

Le martelage de plusieurs tôles lisses à partir de lingots est à la base de la mise en forme des parties en relief des éléments du pavillon. La ciselure est utilisée pour élaborer des détails en relief sur certaines tôles. Des tôles lisses sont également des préproduits pour la crinière, les tubes longs et les ba-

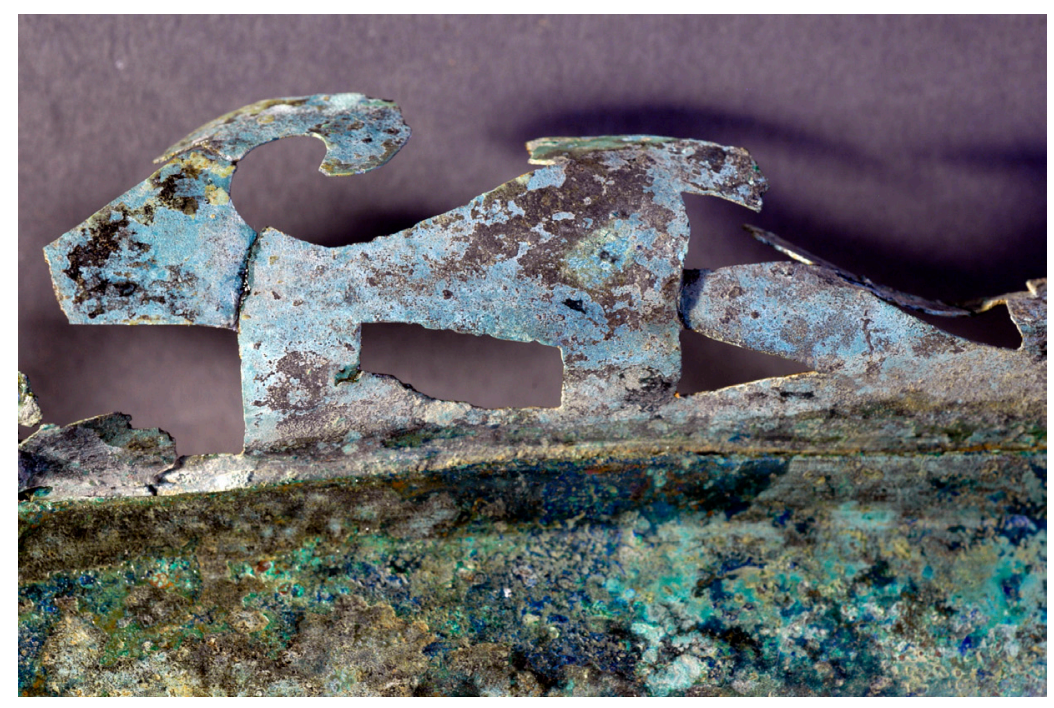

Fig. 40. Décor de la crinière ajouré (cl. B. Armbruster, CNRS).

gues par enroulement et cintrage à partir de tôles rectangulaires. Les contours des tôles sont découpés au ciseau. Le découpage du décor de la crinière termine les travaux sur tôle (fig. 40). L'assemblage mécanique à froid par emboîtage et cerclage précède les assemblages à chaud par brasure. La chaîne s'achève par la finition par polissage de la surface et des jonctions ainsi que par l'incrustation des éléments des yeux. S'il y a eu étamage, il est intervenu après le polissage, et avant l'incrustation.

Les aspects technologiques décrits ici pour ce carnyx sont comparables - aussi bien pour la fabrication des parties martelées et ciselées de la tête, pour les éléments tubulaires puis les pièces coulées, comme pour les anneaux et l'embouchure - avec les morceaux de carnyx trouvés dans le sanctuaire de Mandeure, Doubs (pavillon, anneaux décorés et oreilles) ${ }^{17}$, et également avec les pièces de Sanzeno, Trento (tubes, bagues, anneaux décorés et oreille, embouchure $)^{18}$.
17. Barral 2009.

18- Roncador 2009. 


\section{Étude métallurgique}

(M. Pernot, avec la collaboration de Fr. Adamski, S. Campodonico et P. Piccardo)

\section{L'échantillonnage}

Le carnyx complet est constitué par l'assemblage d'une trentaine de pièces ; pour diverses raisons, c'est un échantillon d'une dizaine des prélèvements qui a été étudié sous l'angle de la métallurgie de fabrication.

Rappelons que, pour l'accès à la composition et à la microstructure du matériau, un prélèvement exploitable doit être réalisé sans le traumatisme d'une déformation ; il doit aussi comporter la plus forte proportion possible de métal sain, donc non corrodé ; en conséquence, la taille requise est de quelques millimètres cubes ${ }^{19}$. En vue de retracer les étapes de la mise en forme, la position du fragment doit être repérée par rapport aux axes principaux de la pièce. Ainsi, chaque prélèvement est obtenu, soit par une rupture fragile au niveau d'une zone corrodée localisée entre des zones où le métal est sain, soit, lorsque le cas précédent ne se présente pas, par découpe avec une scie d'horloger directement dans le métal.

Pour ce qui est de l'objet fini dans son état d'origine, il faut tout d'abord conserver en mémoire que son plan de symétrie (celui que présentent tous les vertébrés) a été utilisé par le concepteur ; sauf pour les extrémités, embouchure et groin, il a choisi de réaliser les segments de tube du cou et la tête formant le pavillon, en deux pièces de tôle, symétriques par rapport à ce plan et donc image l'une de l'autre dans un miroir ; il en est, évidemment, de même pour les oreilles. Afin de limiter les atteintes à l'intégrité de l'objet, dans les segments concernés, une seule des deux pièces en position de symétrie a été soumise à un prélèvement. Le problème n'est, bien sûr, pas semblable pour les bagues d'assemblage des segments; le décor des anneaux massifs respecte la symétrie, mais ils sont en une pièce; la pièce de tôle, placée sous l'anneau et servant réellement de raccord, ne la respecte pas vraiment. Naturellement, le second critère important, pour décider de la stra-

19. Pernot 1999. tégie de l'échantillonnage, est l'état de conservation de chacune des différentes pièces.

Les prélèvements ont été effectués lors de deux campagnes ${ }^{20}$; les caractéristiques de ceux qui se sont révélés être exploitables, sont présentées dans le tableau 1 .

Les prélèvements ont été préparés pour les examens métallographiques et les analyses de composition selon la procédure habituelle ${ }^{21}$. Pour les pièces en tôle, en fonction de la taille et de l'état de conservation, le plan de la section d'observation a été choisi pour certains sur la tranche $(467 \mathrm{~B}-12$, 467B-3, T6, T1), avec parfois deux sections, réalisées à l'aide d'une tronçonneuse à lame diamantée, dans des plans perpendiculaires (467B-4), pour un autre sur la face seulement (467B-11) ; pour un autre encore, deux sections (tranche et face) ont été préparées (467B-8) ; pour le dernier (280) trois sections ont été préparées, l'une sur la face et deux dans des plans perpendiculaires sur la tranche. Les observations suivant plusieurs orientations des sections ont pour but de caractériser la tridimensionnalité de la morphologie des inclusions. Les prélèvements, ou les découpes des prélèvements, ont été enrobés dans une résine adaptée, puis pré-polis avec des papiers abrasifs jusqu'au grain 1000 et enfin polis sur des plateaux tournants avec des pâtes diamantées jusqu'au grain 0,25 $\mu \mathrm{m}$. Les sections ont été observées directement après polissage pour accéder à l'état inclusionnaire, puis après attaque chimique avec une solution alcoolique de perchlorure de fer destinée à révéler les joints des grains et de macles, au moyen d'un microscope optique (MO) et d'un microscope électronique à balayage (MEB) ; les analyses ont été conduites, sur des plages de métal sain, par un dispositif EDXS associé au MEB.

20- Les prélèvements de la première campagne (notés 467B avec un numéro et 280), réalisés pendant le travail de restauration au laboratoire Materia Viva à Toulouse en 2007, ont été étudiés à l'IRAMAT à Bordeaux lors du doctorat de Fr. Adamski (Adamski 2008) ; ce travail a été soutenu par une allocation de recherche cofinancée par le CNRS et par la Région Aquitaine, dans le cadre d'un contrat de recherche, ainsi que par un Programme Collectif de Recherche, financé par le Ministère de la Culture, attribué au travers du SRA d'Aquitaine. Les prélèvements de la seconde campagne (T1, T6 et 2149.467B), réalisés après la restauration en mars 2010 au Musée Saint Raymond lors du démontage de la première exposition, ont été étudiés lors d'un stage par la Dottoressa S. Campodonico, en partie à Bordeaux, et en partie à Gênes, sous la direction de Professeur P. Piccardo durant l'été 2010.

21. Pernot 1999. 


\begin{tabular}{|l|l|l|}
\hline \multicolumn{1}{|c|}{ Localisation des prélèvements } & $\begin{array}{l}\text { Référence } \\
\text { attribuée }\end{array}$ & $\begin{array}{l}\text { Épaisseur } \\
\text { des tôles }\end{array}$ \\
\hline Tôle de droite du pavillon & $467 \mathrm{~B}-12$ & $0,4 \mathrm{~mm}$ \\
\hline $\begin{array}{l}\text { Tôle de la bague de jonction entre le pavillon et le } \\
\text { cou }\end{array}$ & $467 \mathrm{~B}$ - 11 & $\begin{array}{l}\text { non } \\
\text { mesurée }\end{array}$ \\
\hline Tôle de droite du cou & $467 \mathrm{~B}-8$ & $0,5 \mathrm{~mm}$ \\
\hline $\begin{array}{l}\text { Anneau de la bague placée sur le cou (qui ne sert } \\
\text { pas pour un assemblage) }\end{array}$ & $467 \mathrm{~B}$ - 7 & \\
\hline $\begin{array}{l}\text { Tôle de la bague d'assemblage entre le cou et le } \\
\text { premier tube (plus fort diamètre) }\end{array}$ & $467 \mathrm{~B}$-3 & $0,4 \mathrm{~mm}$ \\
\hline $\begin{array}{l}\text { Anneau de la bague d'assemblage entre le cou et le } \\
\text { premier tube (plus fort diamètre) }\end{array}$ & $467 \mathrm{~B}$ - 2 & \\
\hline Tôle ajourée de l'échine & $467 \mathrm{~B}-4$ & $0,3 \mathrm{~mm}$ \\
\hline Tôle du second tube (haut du tube 280) & 280 & $0,6 \mathrm{~mm}$ \\
\hline $\begin{array}{l}\text { Tôle du dernier tube (bas du tube près de } \\
\text { l'embouchure) }\end{array}$ & $280 / \mathrm{T} 6$ & $0,5 \mathrm{~mm}$ \\
\hline $\begin{array}{l}\text { Tôle de l'oreille gauche (bord externe au niveau de } \\
\text { la largeur maximale) }\end{array}$ & $464-1 / \mathrm{T} 1$ & $0,5 \mathrm{~mm}$ \\
\hline Fragment détaché du matériau d'une des brasures & $2149.467 \mathrm{~B}$ & \\
\hline
\end{tabular}

Tableau 1. Caractéristiques des prélèvements du carnyx qui ont pu être étudiés. Les épaisseurs sont mesurées sur les coupes métallographiques observées en microscopie optique.

L'observation au microscope d'une section réalisée sur la tranche d'une tôle permet d'apprécier l'épaisseur conservée ; les mesures qui ont pu être faites sont portées dans le tableau 1. Comme on pouvait s'y attendre, en particulier à partir de ce qui est connu pour les vases, les valeurs se situent entre 3 et 6 dixièmes de millimètre.

Les compositions des alliages

Toutes les tôles examinées, soit huit pièces, ont été réalisées avec des alliages binaires de cuivre et d'étain, donc avec des vrais bronzes. Les analyses indiquent que la teneur en étain varie légèrement d'une pièce à l'autre dans la fourchette de 10 à $13 \%$ en masse. Ces variations ne nous semblent pas être pertinentes ; c'est-à-dire qu'elles représentent une dispersion normale pour des pratiques artisanales, sans être le reflet de choix explicables. Les examens métallographiques montrent la présence d'inclusions de sulfure de cuivre correspondant à une faible teneur en soufre, située approximativement dans la fourchette de 0,2 à 0,4\% en masse, et une absence quasi-totale de nodules de plomb. Dans les conditions d'analyse utilisées, aucune impureté soluble dans l'alliage n'a été détectée. Le cuivre utilisé pour élaborer les alliages est d'une très bonne "propreté"; il ne s'agit pas d'un métal de qualité exceptionnelle, mais de la qualité supérieure du métal courant ${ }^{22}$.

Les analyses des prélèvements des deux anneaux indiquent qu'ils ont été réalisés avec des alliages ternaires de cuivre, d'étain et de plomb. Les teneurs en étain sont situées vers $11 \%$ en masse ; celles du plomb sont entre 10 et $13 \%$ en masse. L'élément soufre est détecté à l'état de traces, mais ne peut être dosé même qualitativement à cause de la présence du plomb ; la présence d'arsenic est détectée et estimée, de manière très approximative, à hauteur de $0,3 \%$ en masse. Le choix d'un bronze au plomb est, sans aucun doute, un acte délibéré ; la présence de traces d'arsenic pourrait indiquer, s'il s'agit bien d'une impureté véhiculée par le cuivre, que les alliages ternaires ont été réalisés avec un cuivre différent de celui employé dans le cas des alliages binaires. Nous ne sommes pas en mesure de proposer une interprétation de ce qui reste, pour l'instant, une hypothèse.

Bien que très corrodé, le fragment de brasure permet d'établir qu'il s'agit d'étain non allié et non, soulignons-le, d'un alliage d'étain et de plomb.

\section{Les procédés de formage}

Les caractéristiques microstructurales observées dans le cas des alliages des pièces en tôle indiquent que les grains sont de formes polygonales, de petites tailles (quelques dizaines de micromètres) et qu'ils sont barrés de larges bandes correspondant à des macles d'origine thermique (fig. 41); il s'agit donc toujours d'un état recristallisé résultant d'un travail de déformation plastique réalisé par plusieurs passes de martelage alternées de recuits de recristallisation. De fines stries, d'orientations diverses et localisées dans la totalité ou une partie des grains, sont observables dans nombre de cas ; ces macles d'origine mécanique indiquent qu'une faible déformation a été appliquée après le dernier traitement de recristallisation. Cette légère déformation est attribuable à la dernière passe de martelage destinée à "tendre les lignes” en même temps qu'à durcir quelque peu le

22- À titre d'exemple, un cuivre de qualité exceptionnelle, avec une teneur en soufre plusieurs dizaines de fois moindre, de l'ordre de 0,01\%, a été employé, vers la fin $\mathrm{du} \mathrm{VI}^{\mathrm{e}} \mathrm{s}$. a.C., pour élaborer le bronze utilisé pour fabriquer la pièce du récipient du cratère de Vix (Pernot 2003). 


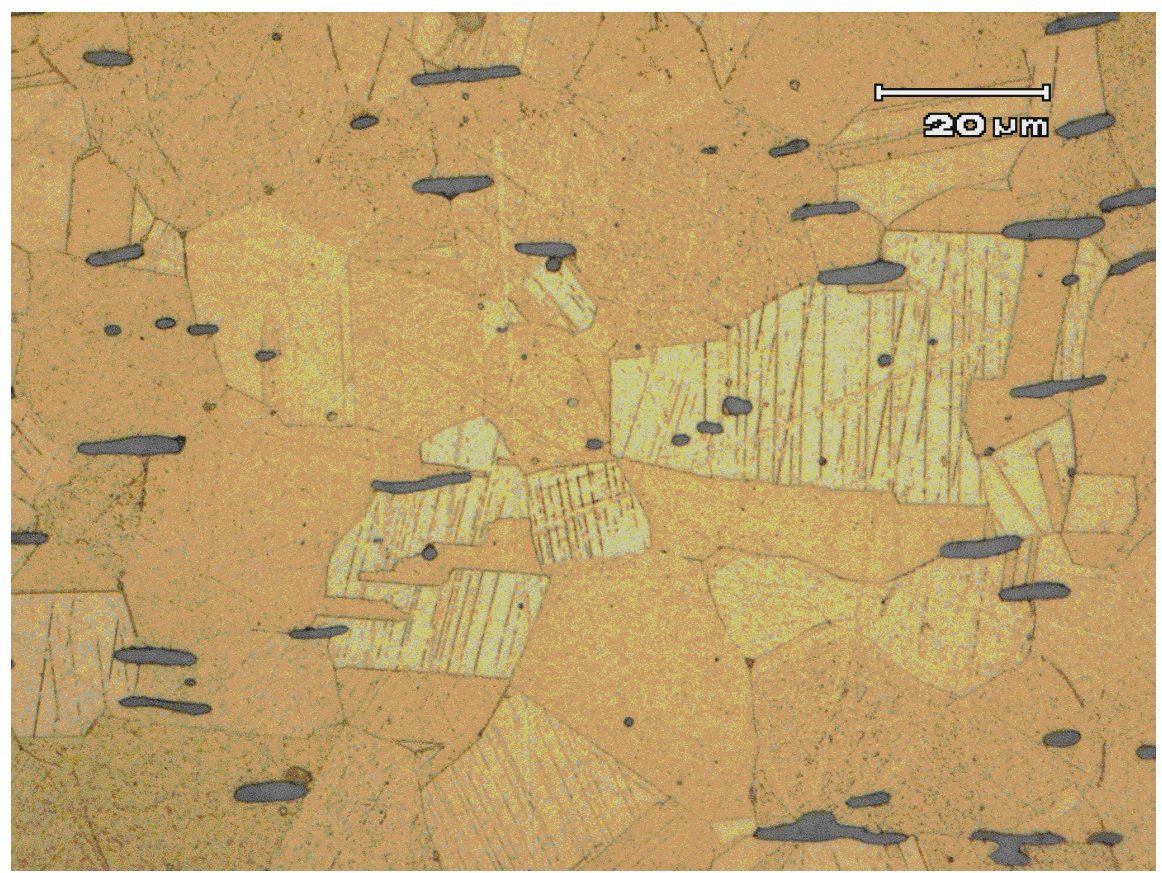

Fig. 41. Section polie et attaquée par une solution de perchlorure de fer alcoolique de la tranche de la tôle du prèlèvement 467B-3 du carnyx, observée en microscopie optique. La microstructure est typique d'un état recristallisé : les grains apparaissent de forme polygonale ; certains d'eux sont barrés par de larges bandes (macles de recristallisation) ; I'allongement des inclusions de sulfure de cuivre (en gris moyen) témoigne du travail total de martelage. La présence de fines stries (macles de déformation) dans certains grains indique qu'une faible déformation a été subie par l'alliage après le dernier recuit.

matériau pour assurer une meilleure résistance mécanique des pièces et donc de l'objet.

Les inclusions de sulfure de cuivre apparaissent le plus souvent de forme allongée (fig. 41) ; ces inclusions non métalliques, qui se déforment sensiblement comme la matrice métallique pendant les passes de martelage mais qui restent insensibles aux recuits, témoignent du travail total de déformation. Les tôles ont, comme habituellement dans le contexte chronologique, été obtenues en amincissant par des passes de martelage - et des recuits - une ébauche venue de fonderie qui, parce que le volume de matière reste constant, a augmenté de surface. L'allongement des inclusions dans différentes directions du plan de la tôle indique que, dans la plupart des cas, le martelage a été conduit de manière multidirectionnelle. Il s'agit très certainement d'un travail où, à chaque passe, le batteur change la direc- tion de l'enchaînement des coups de marteau ; par opposition à un travail qui n'allongerait l'ébauche que dans une seule direction, cette déformation est qualifiée d'expansion biaxiale. Plus de 70 mesures du facteur d'allongement des inclusions de sulfure de cuivre indiquent une valeur moyenne située vers 30 ; en restant dans l'hypothèse d'une expansion biaxiale, un modèle simple ${ }^{23}$ nous permet d'estimer la réduction d'épaisseur, entre l'ébauche et le produit fini, aux environs d'un facteur 10. En d'autres termes, l'épaisseur des ébauches venues de fonderie était située vers $5 \mathrm{~mm}$ pour aboutir à une tôle dont l'épaisseur finale est vers $0,5 \mathrm{~mm}$. Ce schéma de préparation des tôles s'inscrit parfaitement dans le cadre des compétences des batteurs de bronze du

23. Piccardo \& Pernot 1997. 


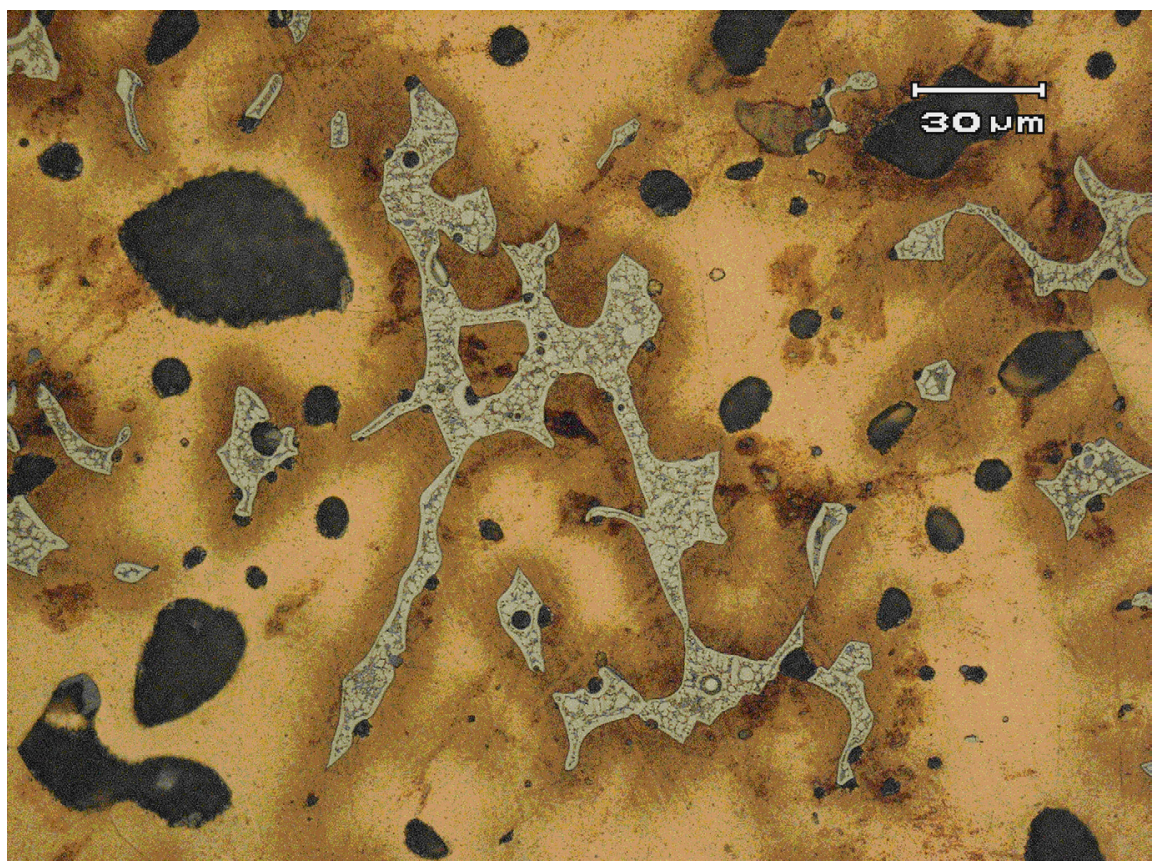

Fig. 42. Section polie et attaquée par une solution de perchlorure de fer alcoolique du prèlèvement 467B-7 du carnyx, observée en microscopie optique. La microstructure de l'alliage ternaire de cet anneau est typique d'un état brut de fonderie : les joints des grains sont sinueux ; la variation de la teneur en étain entre le cœur et la périphérie (ségrégation) est traduite par des nuances du jaune au marron ; les nodules de plomb apparaissent en noir ; les zones claires, riches en étain, présentent la sous-structure caractéristique de la transformation euctectoïde qui a lieu pendant le refroidissement.

$\mathrm{I}^{\text {er }}$ millénaire a.C. Il est également à noter que le choix d'un cuivre de bonne qualité est cohérent avec la recherche d'un alliage qui supporte sans trop de risques de fissuration, donc de rupture rédhibitoire, l'important taux total de déformation plastique. De même, les teneurs en étain choisies conduisent à des alliages d'une couleur dorée qui supportent bien la déformation dans des conditions telles que les passes de déformation ne soient pas trop fortes - moins de $10 \%$ de réduction par passe, surtout au début du travail - et que les recuits soient effectués autour de $600^{\circ} \mathrm{C}$. Il a donc fallu au batteur appliquer entre 10 et 15 passes de déformation et autant de traitements de recuit.

Le faciès de la microstructure observée pour les deux anneaux est semblable. Il présente des grains, de forme dendritique, dont la taille peut atteindre le millimètre ; dans ceux-ci, la ségrégation de l'étain (variation de la teneur entre le coeur - peu riche - et la périphérie - plus riche - des grains) est nettement visible; des nodules de plomb, de taille variée allant jusqu'à une centaine de micromètres, sont présents ; des inclusions métalliques, de forme interdendritique, riches en étain, où la sous-structure, qui mélange intiment les phases $\alpha$ et $\delta$, indique qu'elles sont issues de la réaction eutectoïde qui a lieu à $520^{\circ} \mathrm{C}$ durant le refroidissement. Ce faciès (fig. 42) correspond à des états métallurgiques bruts de fonderie ; le choix d'alliages ternaires de cuivre, d'étain et de plomb est, là encore dans le contexte du $\mathrm{I}^{\mathrm{er}} \mathrm{mil}$ lénaire a.C., en parfaite cohérence avec une mise en forme des pièces directement par fonderie. La forme des anneaux oriente plutôt vers la proposition de l'utilisation d'un procédé à la cire perdue, sans que ce soit une certitude. 
Les brasures et la question d'un éventuel étamage

Les assemblages des différentes pièces de l'objet sont assurés par une technique de brasage ; c'est-àdire par l'apport d'un matériau métallique qui fond nettement plus bas que les pièces à joindre. L'analyse du fragment de brasure (tabl. 1) indique que le métal d'apport n'est pas un alliage, mais de l'étain non allié. Sur une partie des prélèvements examinés, une couche d'étain métallique non allié est observée sur la surface externe des pièces, aussi bien sur des tôles que sur les anneaux. Cette couche présente un faciès très particulier (fig. 43) ; elle n'est pas, par la corrosion entre autres causes, présente partout. La question d'un étamage de certaines pièces, ou de la totalité de l'objet, se pose donc. Pour des raisons évidentes, la grande majorité des prélèvements de la première campagne, réalisés avant restauration, provient de zones qui sont localisées près du bord des pièces, donc dans des endroits proches des assemblages, voire, et c'est le cas des tôles et des anneaux des bagues, dans des pièces qui servent à l'assemblage des segments. La seconde campagne avait pour but de tenter d'approcher la réponse à cette question de l'étamage ; le résultat nous laisse toujours

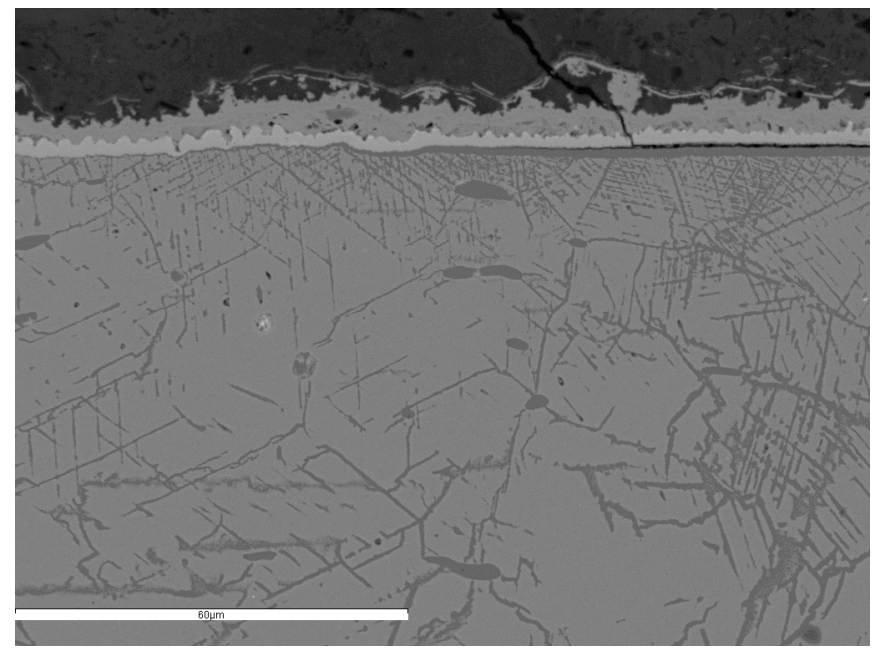

Fig. 43. Section polie de la tranche de la tôle du prélèvement 280 du carnyx observée en microscopie électronique à balayage. La couche d'étain, avec un faciès particulier, apparaît en gris clair au-dessus du bronze. La microstucture du bronze est partiellement révélée par la corrosion ; des inclusions allongées de sulfure de cuivre sont également visibles. Des produits de corrosion recouvrent la couche d'étain (partie haute de l'image) et, en l'absence de nettoyage, la masque complètement. dans l'incertitude car les prélèvements faits l'ont été après le travail de nettoyage qui a pu éliminer cette éventuelle couche d'étain qui, naturellement après l'enfouissement, est le plus souvent interstratifiée dans les produits de corrosion. Dans l'état actuel de l'avancement de l'étude, nous ne pouvons trancher entre deux hypothèses. Soit les brasures d'assemblage débordaient des zones de jonction ; cela était bien sûr visible par le contraste entre la couleur dorée du bronze et celle très blanche de l'étain. Nous devons cependant nous garder de tout jugement sur la qualité de la finition des assemblages ; ce que nous considérerions aujourd'hui comme un défaut de finition ne l'était peut-être pas dans la culture qui a produit et utilisé l'objet. L'autre cas probable est qu'une partie de l'objet, ou bien encore la totalité de la surface visible, était étamée ; l'aspect du produit fini pouvait alors être soit entièrement blanc, soit bicolore avec le contraste entre des plages dorées et des plages "argentées". Nous restons actuellement totalement dans l'incertitude quant à cette caractéristique visuelle de l'objet dans le contexte de son usage. Lors de l'étude et de la restauration des autres objets, la stratégie d'échantillonnage devra être adaptée à ce problème.

\section{Les éléments de comparaison à l'échelle européenne (Chr. Maniquet)}

Les autres trompes connues dans le monde antique

Si l'on exclut les trompes métalliques de l'Inde, de l'Égypte, d'Israël ou d'Assyrie et que l'on resserre notre recherche sur l'Europe, les plus anciennes connues remontent à l'âge du Bronze. En France, deux fragments de cornes courbes en bronze proviennent des dragages de la Loire, près d'Orléans. Aujourd'hui disparues, elles ne conservaient ni embouchure, ni pavillon. En Irlande, sont connues des trompes à embouchure latérale ou terminale.

La trompe la plus spectaculaire de l'âge du Bronze reste néanmoins le lur scandinave, daté entre 1350 et 800 a.C. Plusieurs ont été découverts en Norvège, Suède et Allemagne septentrionale, mais la plupart proviennent de tourbières danoises. Longs de $1 \mathrm{~m}$ à $2,25 \mathrm{~m}$, les lurs, en forme de $\mathrm{S}$ étaient fabriqués à la cire perdue, contrairement aux 
trompes irlandaises en tôle de bronze. Leur pavillon circulaire était orné d'un disque à bossette. Leur sonorité était puissante. Pour en jouer, il faut tenir le lur à bout de bras, le pavillon tourné vers le haut ${ }^{24}$.

Des trompes métalliques contemporaines des carnyx celtiques sont également connues en Grèce, en Etrurie et à Rome. En Grèce, à l'époque classique, la salpinx est constituée d'un tuyau long et mince à perce cylindrique, aboutissant à un pavillon. Les deux parties de l'instrument sont usuellement en bronze. La salpinx du musée de Boston, qui possède une embouchure et mesure $1,55 \mathrm{~m}$ de long, pourrait dater de l'époque impériale. Les représentations sur céramique semblent indiquer que ces instruments étaient tenus inclinés à $45^{\circ}$ vers le bas pour en jouer. L'un des principaux usages de la salpinx est militaire. Cependant, des concours de trompettes étaient organisés aux Jeux olympiques depuis 396 a.C., au cours desquels on jugeait essentiellement la puissance du son émis.

La longue trompette droite à pavillon recourbé dénommée lituus est d'origine étrusque. Son nom est le même que celui du bâton augural de forme proche. Au musée étrusque du Vatican, un lituus en bronze découvert dans une sépulture, mesure 1,60 m de longueur. Deux litui complets ont été découverts dans des cours d'eau allemands, mais, à la différence des litui étrusques, ils sont plus courts, coniques et pourvus de deux oillets de suspension ${ }^{25}$. Le lituus était vraisemblablement joué à l'horizontale ou incliné légèrement vers le bas. L'instrument découvert dans la rivière Witham, à Tattershall Ferry, long de $1,30 \mathrm{~m}$, est interprété par certains comme un lituus plutôt qu'un carnyx.

Chez les Romains, le lituus transmis par les étrusques, conservait sa forme recourbée. Trois autres instruments métalliques étaient employés par l'armée romaine.

La tuba, en bronze, était une trompette droite au corps conique composé de plusieurs pièces assemblées, terminé par un pavillon évasé simple. Sa taille devait atteindre $1,20 \mathrm{~m}$. Sa forme devait lui conférer un son relativement fort et rond, que les auteurs antiques s'accordent à trouver terrifiant.

24- Clodoré 2002, 94.

25. Le carnyx et la lyre, $50, n^{\circ} 38$
La bucina, autre instrument métallique, à embouchure, se différencie de la tuba par une taille vraisemblablement un peu inférieure et un corps étroit jusqu'au pavillon. Le son de cet instrument, plus perçant que celui de la tuba est souvent qualifié de rauque $^{26}$.

Bucina et tuba, pour en jouer, devaient être tenues horizontalement, ou légèrement incliné vers le haut.

Le dernier instrument, le cornu, s'apparentait à un grand cor circulaire. L'épigraphie a permis de certifier que ces trois instruments pouvaient aussi être utilisés par la cavalerie. Leur fonction principale était de transmettre les ordres aux soldats dans le feu de la bataille ${ }^{27}$.

La trompe droite, la tuba, fut introduite en Gaule sous l'influence des Romains. Alors que seulement quelques fragments ont été découverts dans tout l'empire romain, des exemplaires presque complets ont été découverts à Neuvy-en-Sullias (Loiret) et Saint-Just-sur-Dive (Maine-et-Loire) dans des contextes vraisemblables de sanctuaires. D'après les recherches récentes, ces deux trompettes devaient être longues à l'origine de $1,70 \mathrm{~m}$ à $1,90 \mathrm{~m}^{28}$.

C'est donc dans cette longue tradition d'instruments métalliques que s'intègrent les carnyx, sans doute inspirés au départ des modèles utilisés par les civilisations voisines.

Les éléments de carnyx identifiés dans le monde celtique

\section{Les pavillons}

Hormis ceux de Tintignac, seuls deux autres pavillons de carnyx sont connus dans le monde celtique. L'un d'eux, découvert en 1816 à Deskford en Écosse ${ }^{29}$, représente une tête de sanglier en tôle de bronze martelée datée de la fin du I $\mathrm{I}^{\mathrm{er}} \mathrm{s}$. ou du $\mathrm{II}^{\mathrm{e}} \mathrm{s}$. p.C. Quasiment tous les auteurs s'accordent sur l'idée qu'il était utilisé par les tribus calédoniennes dans une région située hors de l'influence romaine,

26- Vincent 2007, 35.

27- Vincent 2007, 36.

28. Vendries 2007, 131-132.

29. Piggott 1959, 24-29 ; Hunter 2001, 78-85. 
mais s'agit-il vraiment d'un instrument de musique $^{30}$ ?

L'autre pavillon, découvert en 1883 sur le sanctuaire de Mandeure (Doubs), présente en revanche des ressemblances indéniables avec les nôtres ${ }^{31}$. Comme sur les carnyx de Tintignac, de longues nervures courbes ont été martelées le long de la mâchoire de l'animal et une pointe sur le maxillaire figure la canine éversée. L'emplacement de l'œil et le trou circulaire destiné à recevoir l'oreille sont bien visibles. La datation de ce pavillon reste incertaine. Celle du dépôt dans lequel il se trouvait est estimée entre le $\mathrm{I}^{\mathrm{er}}$ s. a.C. et le $\mathrm{I}^{\mathrm{er}}$ s. p.C.

En ce qui concerne les boutoirs, sur les sangliers en tôle de bronze de Neuvy-en-Sullias ou de Soulacsur-Mer ${ }^{32}$, ils se présentaient sous la forme d'un disque sur lequel les naseaux sont figurés. Le seul élément qui a pu appartenir à une trompette celtique demeure un disque de tôle découvert à Mandeure, sur lequel les deux naseaux étaient fortement marqués en creux ${ }^{33}$. Cette pièce, déconnectée de toute autre, a pu néanmoins également appartenir à une enseigne...

Les oreilles

La découverte de Tintignac a permis la réinterprétation d'objets trouvés anciennement dans le territoire celtique. Il s'agit en particulier des grandes feuilles en tôle de bronze qui s'avèrent être des oreilles de trompettes ${ }^{34}$.

30- Le "pavillon de carnyx" de Deskford a été découvert en 1816 en Écosse, dans une tourbière (Piggott 1959, 24-29; Hunter 2001, 78-85). Représentant une tête de sanglier stylisée en tôle de bronze martelée, la tête d'animal était dotée à l'origine d'une langue en bois mobile. Ce "pavillon" ne ressemble guère à ceux de Tintignac ou de Mandeure. Aucun fragment de tube ne l'accompagnait. Son identification comme carnyx émise dès l'origine, n'a quasiment jamais été remise en question. $\mathrm{Ne}$ pourrait-on voir dans cet objet, à l'instar de Michel Feugère, une enseigne de type draco brandie par les Daces, les Sarmates ou les Parthes (Feugère 1993, 58 ; Lebedynsky 2010) ? Les monuments représentent ce draco par une étoffe fixée sur une tête d'animal à la gueule ouverte qui, dans la marche, portée au sommet d'une hampe, se gonflait et se déroulait au vent comme les replis d'un serpent.

31- Barral et al 2007, 388 ; Le cheval et la danseuse 2007, cat. $38.1,77,226$.

32. Moreau et al. 1995.

33. Le cheval et la danseuse 2007,227 , cat. 38.4 .

34- Maniquet 2008, 312
La question de la latéralité tant pour celles de Tintignac que pour les autres, s'est posée. La réponse ne pouvait être obtenue que par l'observation de la seule oreille en connexion identifiée à ce jour, celle du carnyx 256 (oreille droite). Quand on regarde l'animal de face, la gorge médiane présentant logiquement son profil concave, son extrémité se dirige vers le haut et le bord rectiligne se situe en haut. Si cette disposition était toujours respectée, alors les éléments 460 et 464 sont des oreilles gauches et les fragments 326 et 466 seraient des fragments d'oreille droite. La pièce 466 présente une particularité dans la mesure où le bord sinueux se retrouverait tourné vers le haut.

On peut, à partir de ces observations, assurer aujourd'hui que celle découverte à La Tène (Suisse) est une oreille gauche ${ }^{35}$; celles de Abentheurer Hütte (Allemagne) correspondent à une paire qui a pu appartenir au même instrument ; à Mathay-Mandeure (Doubs), on a affaire à une oreille gauche et quatre droites. On ne reviendra pas ici sur les différences de dimensions entre ces dernières et l'ensemble des autres feuilles reconnues à Tintignac et ailleurs.

On ajoutera à celles déjà citées, celle mise au jour en 1950 à Sanzeno $\left(\right.$ Italie) ${ }^{36}$, bien conservée et récemment restaurée, dont la longueur conservée atteint $26,7 \mathrm{~cm}$ et la largeur $13,1 \mathrm{~cm}$. Il s'agit là encore d'une oreille droite.

\section{Les crêtes}

La découverte de plusieurs fragments de crêtes dans le dépôt de Tintignac et de celles de Soulacsur-Mer, du Gué-de-Sciaux ${ }^{37}$ ou de Mandeure $^{38}$ permet d'attester que cette tôle, en alliage cuivreux ou en fer, était, sur le carnyx présenté ici, doublée en périphérie d'un orle métallique qui n'était plus en

\footnotetext{
35- Vouga 1923, pl. XIX.

36- Roncador 2009, 552-553. Le site de Sanzeno, dans la partie septentrionale du Val di Non, en Italie septentrionale, est considéré comme un important centre de production. Pendant les recherches à Sanzeno du Prof. G. Fogolari, furent retrouvés une "lame de bronze à la forme de feuille lancéolée avec une grosse nervure vide au revers, longue de. $30 \mathrm{~cm}$, et des éléments de tuyau en bronze faisant partie des mêmes complexes". Rosa Roncador, suite à la découverte de Tintignac a extrait ce mobilier de leur réserve et a ainsi permis d'y reconnaître une oreille, trois fragments de tubes et une embouchure (Roncador 2009).

37- Le cheval et la danseuse 2007, 79, fig. 28.

38- Barral 2009, 193, fig. $8 / 1$ et $8 / 2$.
} 


\begin{tabular}{|l|l|l|l|l|}
\hline \multicolumn{2}{|c|}{ Les oreilles } & \multicolumn{1}{c|}{ Longueur } & \multicolumn{1}{c|}{ Largeur } & \multicolumn{1}{c|}{ Latéralité } \\
\hline Tintignac 326 & Incomplète & Sup à $160 \mathrm{~mm}$ & $130 \mathrm{~mm}$ & Droite \\
\hline Tintignac 377 & Incomplète & Sup à $300 \mathrm{~mm}$ & $165 \mathrm{~mm}$ & Droite \\
\hline Tintignac 460 & Quasi-complète & Sup à $410 \mathrm{~mm}$ & $200 \mathrm{~mm}$ & Gauche \\
\hline Tintignac 464 & Complète & $370 \mathrm{~mm}$ & $160 \mathrm{~mm}$ & Gauche \\
\hline Tintignac 466 & Incomplète & Sup. à $208 \mathrm{~mm}$ & $90 \mathrm{~mm}$ & Droite \\
\hline Sanzeno & Quasi-complète & $267 \mathrm{~mm}$ & $131 \mathrm{~mm}$ & Droite \\
\hline Mandeure 1 & Quasi-complète & $125 \mathrm{~mm}$ & $72 \mathrm{~mm}$ & Gauche \\
\hline Mandeure 2 & Quasi-complète & $118 \mathrm{~mm}$ & $68 \mathrm{~mm}$ & Droite \\
\hline Mandeure 3 & Incomplète & $134 \mathrm{~mm}$ & $80 \mathrm{~mm}$ & Droite \\
\hline Mandeure 4 & Complète & $114 \mathrm{~mm}+$ douille $50 \mathrm{~mm}$ & $55 \mathrm{~mm}$ & Droite \\
\hline Mandeure 5 & Complète & $153 \mathrm{~mm}+$ douille $75 \mathrm{~mm}$ & $80 \mathrm{~mm}$ & Droite \\
\hline La Tène & Complète & $480 \mathrm{~mm}$ & $190 \mathrm{~mm}$ & Gauche \\
\hline Abentheurer Hütte 1 & Complète & 415 & $174 \mathrm{~mm}$ & Gauche \\
\hline Abentheurer Hütte 2 & Incomplète & $?$ & $?$ & Droite \\
\hline
\end{tabular}

connexion et qui n'a pas été identifié parmi les objets extraits du dépôt. Malheureusement, à Tintignac comme ailleurs, on ne dénombre que des parties distales de crête. Seul un fragment provenant de Mandeure peut, à ce jour, être interprété comme une portion proximale. Ces crêtes sont souvent comparées à celles des enseignes, assez semblables, simples tôles ajourées en position verticale, la seule complète étant celle du sanglier de Soulac-sur-Mer. On constate que les crêtes de carnyx présentent une courbure beaucoup plus régulière et accentuée que celles des enseignes, à condition qu'elles soient conservées sur une longueur suffisante. À ce titre, la crête du sanctuaire du Gué-de-Sciaux à Antigny (Vienne), bien qu'en fer, s'apparente davantage à une partie d'instrument de musique.

\section{Les embouchures}

L'embouchure de carnyx de Tintignac était la seule reconnue jusqu'alors. Elle doit désormais servir de référence pour en identifier d'autres, mises au jour, parfois anciennement, en contexte archéologique. C'est le cas en particulier d'une pièce métallique découverte en 1950 à Sanzeno ${ }^{39}$.

\begin{tabular}{|l|l|l|l|}
\hline Les embouchures & Longueur & \multicolumn{1}{|c|}{$\begin{array}{c}\text { Diamètre } \\
\text { extérieur du bord }\end{array}$} & $\begin{array}{c}\text { Diamètre à l'extrémité } \\
\text { de la queue }\end{array}$ \\
\hline Tintignac & $111 \mathrm{~mm}$ & $35,5 \mathrm{~mm}$ & $25,6 \mathrm{~mm}$ \\
\hline Sanzeno & $92 \mathrm{~mm}$ & $37 \mathrm{~mm}$ & $27 \mathrm{~mm}$ \\
\hline
\end{tabular}

39. Roncador 2009, 552-553.
Notre embouchure ne dispose pas de bassin, ni donc de grain, ce qui la rapproche de celles des cors. Certaines trompes gallo-romaines, que ce soient les cornua ou les tubae $e^{40}$, possèdent des embouchures dotées de cette cuvette dont la largeur et la profondeur font varier le timbre de l'instrument et la difficulté à jouer des notes graves ou aiguës. Le grain, quant à lui, plus ou moins large, influe sur le débit de l'air propulsé dans l'instrument.

Les lurs scandinaves ne possédaient pas tous le même type d'embouchure ${ }^{41}$. Alors que certaines ressemblent déjà à celles des trompes romaines, d'autres, comme celle découverte à Gullakra, au sud de la Suède, se rapproche davantage de celle de Tintignac (fig. 44).

\section{Le carnyx : une trompe naturelle (Chr. Maniquet)}

Le carnyx fait partie des instruments à vent (ou aérophones), de la sous-famille des cuivres définie par la présence d'une embouchure ${ }^{42}$. Alors que chez les bois, la vibration est créée par une anche, chez les cuivres c'est l'instrumentiste qui la crée avec ses lèvres (le buzz). Aujourd'hui, parmi les cuivres joués, on peut citer la trompette, le cornet à pistons, le cor, le trombone, le saxhorn, le tuba, les instruments d'ordonnance (clairon, trompette de cavalerie, ...),

40- Telles que celles de Neuvy-en-Sullias (Loiret), du VieilÉvreux, de Vieux (Calvados) ou encore des Tournelles (Oise) (Vendries 2007).

41- Schween 2000.

42- Stauder 1963. 

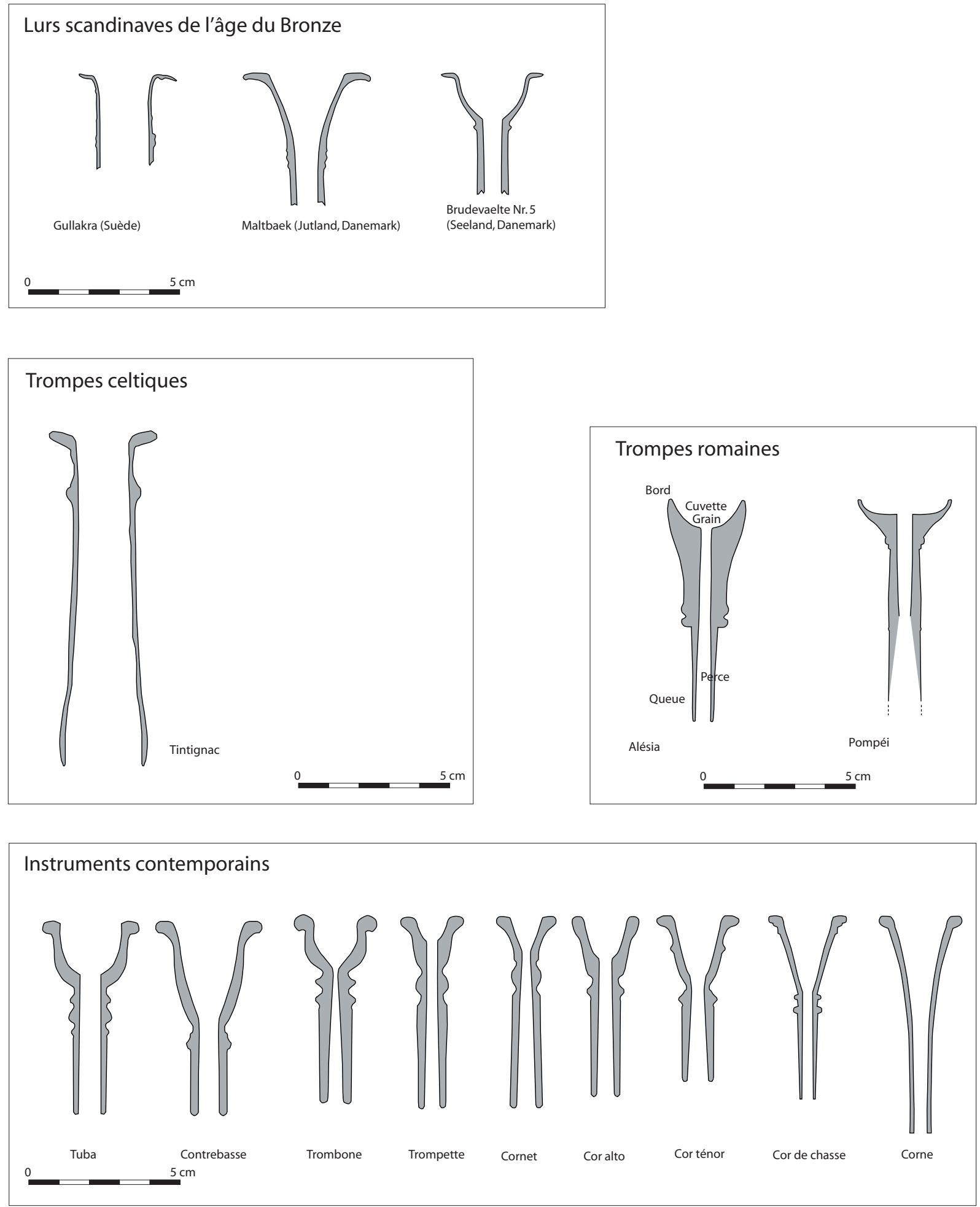

Fig. 44. Comparaison de l'embouchure de carnyx de Tintignac avec les embouchures d'autres instruments de musique, anciens ou récents (dessin Chr. Maniquet, INRAP). 
des cuivres anciens en bois (cornet à bouquin, serpent) ou des instruments "populaires" (cor des Alpes, didgeridoo). Le carnyx ne possède aucun système de coulisse ou de pistons et est de ce fait un cuivre dit "naturel" 43 . L'invention des pistons (vers 1820) a permis de développer la technique instrumentale : le musicien ne produit plus les sons uniquement avec les lèvres mais avec l'intervention des doigts. On parle alors d'instrument chromatique.

Les lèvres, en contact avec l'embouchure sont plaquées contre celle-ci lors du passage de l'air. La vibration acoustique, plus ou moins rapide selon la vitesse de vibration des lèvres, est générée à l'entrée de l'embouchure. Cette vitesse de vibration des lèvres fixe la fréquence fondamentale (en hertz) et donc la hauteur perçue de la note jouée. La forme et la taille des différentes parties de l'instrument (y compris l'embouchure) ont une incidence directe sur la vibration et donc sur le son produit. La géométrie interne de l'instrument, appelée "perce", détermine la majeure partie des propriétés acoustiques de l'instrument. Notons que la largeur et le profil des bords de l'embouchure assurent le confort du musicien pour une plus grande facilité dans le jeu.

La perce du carnyx étant conique en s'élargissant sur toute sa longueur, on associe cet instrument aux cuivres "doux" de perce majoritairement conique (tels les cors, les bugles et les cornets à pistons) qui produisent des sons plus feutrés, plus doux, moins brillants que les cuivres clairs dont la perce est pour une large part cylindrique (les portions cylindriques favorisent la production de sons très brillants, sons dits "cuivrés"). Bien que le bord de l'embouchure du carnyx soit d'une largeur voisine de celui du tuba, la géométrie interne, la perce et la longueur de l'instrument le rapproche plutôt du cor (fig. 44).

L'instrument joue le rôle de résonateur acoustique, le son y est "piégé" et va résonner à des fréquences bien particulières, dites fréquences de résonance. Elles sont déterminées par les dimensions internes (la perce) de l'instrument. Ces fréquences

43. "ce ne sont pas des instruments chromatiques comme le sont nos cors, trompettes ou trombones dotés de pistons ou de coulisses qui permettent d'allonger ou de raccourcir à volonté la colonne d'air. En l'absence de tels perfectionnements, les trompes antiques ne peuvent émettre que des harmoniques naturels plus ou moins importants selon la longueur de la colonne d'air qui est invariable : ce sont donc des "trompes naturelles" (Vendries 2007, 134) déterminent la série de notes jouables par l'instrumentiste. Et si l'instrument est bien construit, la série de notes se rapproche d'une série harmonique. Notons que seule une faible part de l'énergie sonore piégée dans l'instrument est transmise vers le milieu extérieur ambiant via l'extrémité ouverte du pavillon.

À partir de la note la plus grave de la série, la note fondamentale, il est possible de produire les notes plus aiguës, souvent appelées harmoniques ${ }^{44}$ par les musiciens. Le musicien sélectionne la note de la série qu'il souhaite, en contrôlant son "masque" (tension des lèvres et des muscles environnant, surpression dans la bouche). Lorsque les lèvres sont contractées en vue de l'émission d'une note aiguë, la surpression d'air nécessaire pour déclencher la vibration peut être très élevée, jusqu'à 0,1 bar.

En conclusion, on peut attendre du carnyx un son assez semblable à celui du cor naturel, instrument duquel il se rapproche le plus par son embouchure et sa perce. Il devait produire un son plus doux et moins brillant que la trompette. La taille de l'instrument et la forme large et évasée du pavillon laissent supposer un son grave et puissant. En revanche, la présence d'oreilles par lesquelles l'air du tube s'évacue devait sans doute moduler le bruit émis. C'est une spécificité du carnyx qu'on ne rencontre pas chez les autres cuivres naturels. Seule la reconstitution d'un instrument à l'identique, permettra d'en caractériser les effets par l'écoute et l'analyse de sons produits par un cuivriste, et par des mesures acoustiques (mesure des fréquences de résonance par exemple $)^{45}$.

44- Le terme "harmonique" est utilisé ici de manière abusive, car en toute rigueur harmonique signifie "multiple de" : les harmoniques de $100 \mathrm{~Hz}$ sont les multiples de $100 \mathrm{~Hz}$, à savoir $100 \mathrm{~Hz}, 200 \mathrm{~Hz}, 300 \mathrm{~Hz}, 400 \mathrm{~Hz}, 500 \mathrm{~Hz}$ etc ...Or les fréquences de résonances de l'instrument sont au mieux proches d'une série harmonique!

45- Gilbert 2000 ; nos remerciements sincères, pour la relecture de ce chapitre, s'adressent à Joël Gilbert, Directeur de recherche CNRS, Laboratoire d'Acoustique de l'Université du Maine UMR CNRS 6613 (Le Mans). Les premiers résultats de l'étude acoustique menée sur le carnyx ont été présentés à Nantes par J. Gilbert dans le cadre du $11^{\text {e }}$ Congrès Français d'Acoustique, du 23 au 27 avril 2012 : J. Gilbert, E. Brasseur, J.-P. Dalmont, C. Maniquet, Acoustical evaluation of the Carnyx of Tintignac Proceedings of Acoustics 2012, Nantes, April 2012. 


\section{La position de jeu du carnyx (Chr. Maniquet)}

On peut s'interroger sur la position adoptée par les musiciens pour utiliser de tels instruments. Deux éléments peuvent aider à l'appréhender : les représentations de l'instrument en cours d'utilisation et sa morphologie même.

\section{Les représentations}

Le carnyx n'apparaît presque jamais en position de jeu. Sur quelques monnaies, il est représenté seul, ou bien associé à un cheval. En revanche, il est souvent intégré au sein d'un amas d'armes ou d'un trophée, aussi bien sur des monnaies que sur des monuments. Parfois, il est tenu par un personnage vu en pied, mais pas en position de jeu. Un autre type de représentation monétaire montre un cavalier juché sur son cheval brandissant un carnyx. Ce sont principalement des monnaies celtiques découvertes en Angleterre. Parfois, le cavalier tient le carnyx au milieu du tube et le brandit haut, presque horizontalement au-dessus de sa tête. Sur un statère d'Epillus, le cavalier tient le carnyx incliné appuyé contre son épaule, le pavillon orienté vers le haut. Enfin, sur le revers d'un bronze CONNO EPILLOS/ SEDVLLVS ${ }^{46}$, le cavalier tient une enseigne gauloise en position verticale ; derrière lui, deux têtes de sanglier, hures vers le haut, pourraient correspondre aux pavillons de deux carnyx attachés horizontalement ou suspendus sur le cheval. Cependant toutes ces représentations, bien qu'intéressantes du point de vue de la morphologie de l'instrument ou de son transport, n'apportent guère de renseignements sur son utilisation.

Un premier aperçu de la position de jeu peut être reconnu sur les monnaies Lémovices en argent dites "au carnyx" 47 . Elles sont connues par une quinzaine d'exemplaires, dont neuf ont été découverts dans le sanctuaire de Tintignac. D'autres proviennent de Malemort-Cazaudet ${ }^{48}$, de la région de Tulle, des environs de Tintignac, ou encore du Gué de

46- $\quad$ LT XIV, 4578.

47- LT XIII 4551-2, BN 4551-3, Depeyrot 2004, n³5, DT III $n^{\circ} 3393$.

48- Geneviève 2007, 138-139.
Sciaux ${ }^{49}$. Le revers porte un cheval à la crinière perlée, galopant à gauche. En arrière de celui-ci se trouve un carnyx en position verticale, d'où sort une volute qui semble rejoindre le museau du cheval. Sous ce dernier est placée une tête, celle du joueur de carnyx. L'embouchure (droite) du carnyx se trouve à peu de distance et à la hauteur de sa bouche. Sur la monnaie LT 4552, si le dessin est exact, la partie supérieure du corps et le bras gauche du personnage apparaissent. Sa main agrippe le carnyx dans sa partie inférieure, toujours sous le cheval, mais il ne joue pas de l'instrument.

On notera au passage l'importance du carnyx dans le centre-ouest de la France et principalement sur les territoires lémovice et picton, sous la forme de représentations monétaires.

En fait, la seule représentation où le carnyx apparait en fonction, se trouve sur le chaudron de Gundestrup. Sur l'une des plaques de métal interne, trois joueurs de carnyx suivent vers la gauche un groupe formé d'un homme, de six fantassins armés et d'un chien dressé sur ses pattes postérieures. Audessus, et séparé d'eux par un rameau fleuri, deux groupes de deux cavaliers se dirigent vers la droite, derrière un serpent à tête de bélier. À l'extrême gauche, un individu occupant toute la hauteur de la plaque, saisit par la taille et par une jambe un petit personnage qu'il semble vouloir plonger tête la première dans un grand récipient. Les joueurs de carnyx, debout, maintiennent de leur seul bras gauche la base de l'instrument au-dessus de l'embouchure et relèvent légèrement la tête pour souffler dedans. Ils semblent souffrir de prognathie (avec un profil en galoche et le menton en avant). Les embouchures des instruments sont droites pour celui de droite, et paraissent très légèrement recourbées pour les deux autres. On ne peut cependant se baser uniquement sur cette image pour définir la forme de l'instrument. En effet, les personnages n'étant pas représentés de façon très réaliste (un seul bras partant du milieu du corps), il est peu probable que les instruments le soient. On notera cependant que ces derniers sont bien de la même taille que les musiciens, qu'ils sont dotés de gueules d'animaux (entre le sanglier et le cheval) grandes ouvertes avec des petites oreilles et une crête courant le long du cou

49. Bertrand \& Maguer 2007, 193-194. 
jusqu'entre les oreilles. Si l'on considère que la main des musiciens dissimule la première bague d'assemblage, le nombre total de tubes correspond à celui du carnyx de Tintignac. Seul manque l'anneau en partie médiane du cou.

\section{La morphologie}

La morphologie de l'instrument elle-même peut donner des informations quant à la position de l'instrument. Soit il est posé au sol comme le didgeridoo, soit en position horizontale comme la tuba, la bucina ou la trompette actuelle, soit dans la position verticale représentée sur les monnaies lémovices ou le bassin de Gundestrup. Dans le premier cas, si l'on veut orienter le pavillon vers le haut, la crête, fragile, ne peut pas reposer directement sur le sol. Le pavillon dirigé vers le sol n'est pas non plus envisageable. Si l'instrument était tenu horizontalement, le poids du pavillon, relativement lourd, devait entraîner des contraintes mécaniques dangereuses. En outre, dans ce cas, le pavillon doit être orienté vers le haut, le bas ou latéralement. Or, tous les aérophones pourvus d'un pavillon dirigent ce dernier vers le haut ou vers les auditeurs ${ }^{50}$. Pour résoudre ces difficultés, la seule tenue de l'instrument qui paraisse cohérente pour en jouer est bien la position verticale ou proche de la verticale.

Le carnyx étant doté d'une embouchure droite et s'il était bien tenu verticalement, l'instrumentiste devait pencher la tête fortement en arrière. Ceci permettait de créer une colonne d'air se propageant de façon linéaire depuis les poumons du joueur jusqu'au pavillon. La position n'est guère reposante, mais elle autorise un certain équilibre naturel de l'instrument sur la bouche du joueur, les bras maintenant simplement la trompe pour l'empêcher de basculer. En outre, rien n'indique que des "concerts" étaient donnés avec ces aérophones. Ils ont pu, tout comme les instruments utilisés au sein de l'armée romaine, servir à sonner brièvement, mais puissamment, pour transmettre un signal ou des ordres.

Comment le carnyx se transportait-il ? Etait-il partiellement démontable? On peut en effet imaginer qu'il pouvait se scinder en trois parties : l'em-

50. Farkas 1981. bouchure, la partie droite du tube et le pavillon lié à la portion courbe du tube. Aucun anneau, aucun élément métallique ne semble destiné à transporter ou à suspendre cet instrument. Cependant, des lanières en cuir pouvaient éventuellement jouer ce rôle.

\section{Le caractère guerrier et religieux (Chr. Maniquet)}

D'après les textes classiques de Diodore et de Polybe, les trompes contribuaient au tumulte guerrier. Les monnaies les montrent à plusieurs reprises brandies par un cavalier. Ces trompes étaient-elles utilisées lors des combats principalement par les cavaliers? Ceci est envisageable. Le son émis est censé impressionner et intimider l'adversaire avant l'assaut, mais il est fort probable que son bruit puissant ait aussi servi à donner des ordres au cours du combat, comme dans toutes les armées, audibles même au cœur de la cohue de la bataille. On peut même imaginer un code sonore bien établi que chacun des guerriers savait interpréter. En quelque sorte cet instrument a pu servir d'enseigne sonore. On peut dès lors se demander si ces instruments de grande taille, donc visibles de loin et dotés à leur sommet d'une représentation de sanglier n'ont pas assuré dans le même temps le rôle d'enseigne visuelle, au sens habituel. "L'existence d'enseignes militaires, qui implique une organisation de l'armée en unités qui se regroupaient autour de ces insignes, est largement attestée chez les Celtes par les textes depuis le III ${ }^{\mathrm{e}}$ s. av. J.-C.”51. D'après Alain Deyber, “ [...] ces enseignes étaient portées par des hommes de pied, exceptionnellement par des cavaliers" ${ }^{52}$.

Le caractère religieux des enseignes guerrières est indéniable. Selon certains auteurs classiques, dont Polybe $^{53}$, les Gaulois, comme les Romains, stockaient les enseignes sur les sanctuaires par temps de paix. On peut dès lors imaginer qu'elles étaient utilisées lors des processions rituelles guerrières qui s'y déroulaient périodiquement. Les carnyx, au même titre que les enseignes, pouvaient faire partie de ces objets sacrés.

\footnotetext{
51- Kruta 2000, 598.

52. Deyber 2009, 133.

53. Pol., Hist., 2.32.
} 
On peut imaginer en fait que tous les objets que renfermait le dépôt de Tintignac étaient utilisés à l'origine au cours de cérémonies rituelles menées dans le sanctuaire. Ces dernières pouvaient être dirigées par un clergé armé et casqué et rythmées par le son des trompettes. De telles assemblées sont connues sur les sanctuaires, en particulier grâce au texte de Hirtius, dans le livre VIII de la Guerre des Gaules, qui indique que les Bellovaques, suite à la mort de Corréos et la perte de leur cavalerie, convoquent une assemblée au son des trompettes et proclament qu'il faut envoyer à César des députés et des otages ${ }^{54}$.

L'aspect cultuel des instruments de musique n'est cependant pas nouveau, même si les découvertes dans les sanctuaires gaulois ou gallo-romains restent rares ou si leurs contextes sont mal cernés. On rappellera à ce titre la découverte des éléments de carnyx dans un espace cultuel à Mandeure, et sans doute également à Sanzeno. Les trompes romaines de Neuvy-en-Sullias, de Saint-Just-sur-Dive et le pseudo-instrument de Klein-Winternheim (Germanie supérieure) ont, eux aussi, été découverts (au XIX s.) dans des contextes vraisemblablement religieux. Des embouchures d'instrument matérialisent à elles seules, selon Christophe Vendries, le souffle et le son de l'instrument à vent ${ }^{55}$. Certaines ont été découvertes sur le site des Tournelles, dans la forêt de Compiègne, à proximité immédiate du sanctuaire du Vieil-Évreux ou encore dans le sanctuaire des eaux de Sceaux-en-Gâtinais. D'autres proviennent d'une insula abritant un secteur cultuel de la ville de Vieux et d'un temple gallo-romain d'Augst (Suisse). H.-P. Eydoux et A. Reinach ont considéré que la trompette de Neuvy-en-Sullias avait pu sonner lors des processions religieuses ${ }^{56}$.

La crête, le boutoir, les défenses indiquent clairement, malgré une stylisation extrême de la gueule de l'animal, que l'artisan a voulu représenter par la

54- BG, 8.20. "Les Bellovaques et les autres peuples voient soudain arriver, en petit nombre et blessés, les quelques fuyards que les bois avaient préservés du massacre : devant un malheur aussi complet, apprenant la défaite, la mort de Corréos, la perte de leur cavalerie et de leurs meilleurs fantassins, ne doutant pas que les Romains n'approchent, ils convoquent sur-le-champ l'assemblée au son des trompettes et proclament qu'il faut envoyer à César des députés et des otages".

55- Vendries 2007, 139

56- Reinach 1909, 524. partie supérieure de la trompette un sanglier furieux, sur le point de se lancer dans l'affrontement. En outre, l'emploi du carnyx comme signe de ralliement visuel et sonore expliquerait pour partie la représentation de cet animal, souvent utilisé pour les enseignes (telles celles de Soulac-sur-Mer, Gironde), Ilonse (Alpes-Maritimes) ou encore Naintré (Vienne).

Cet animal qui prend une valeur symbolique au moins à partir de l'époque laténienne, représente la "vertu virile", l'agressivité, l'impétuosité guerrière, la force, la ténacité, la vivacité, la puissance, le courage et l'intelligence, en bref les vertus des combattants intrépides. Le sanglier est également, pour certains, la représentation de la classe sacerdotale ${ }^{57}$ : il symboliserait alors le dieu souverain Lug que les Romains assimilent à Mercure. Il est, comme le druide, en rapport étroit avec la forêt et avec l'au-delà. Il matérialise l'immortalité de l'âme. Selon Jacques Santrot, "le sanglier pourrait être considéré comme un animal emblématique, totémique de la nation gauloise" ${ }^{58}$.

Les oreilles du carnyx possédaient à n'en pas douter un rôle acoustique qui reste à définir. Leurs dimensions démesurées restent cependant étonnantes, tout comme leur forme de feuille d'arbre, très différentes des écoutes des sangliers. On peut se demander si l'on n'a pas voulu délibérément représenter des feuilles. Ceci n'est pas sans rappeler le mythe pancelte de la "forêt combattante" qui évoque un combat entre les démons de l'Autre Monde, au cours duquel la végétation elle-même aurait été mobilisée contre eux et que l'on retrouve dans la mythologie irlandaise ${ }^{59}$. Les carnyx avançant vers l'ennemi pouvaient évoquer cette forêt et symboliser la victoire des dieux sur l'Autre Monde représenté par l'adversaire.

On rappellera que les princes celtes du début de la période de La Tène étaient affublés, d'après la statuaire sensée les représenter, d'un couvre-chef en forme de feuilles de gui (statues du Glauberg (Hesse, Allemagne), de Heidelberg (Bade-Wurtemberg), stèles piliers de Holzgerlingen (Wurtemberg), de Pfalzfeld (Rhénanie), double tête de Roquepertuse (Velaux, Bouches-du-Rhône), phalères de Horovicky

\footnotetext{
57- Le Roux \& Guyonvarc'h 1990, fig. 8.

58- Santrot et al. 1996, 291.

59. Sterckx 2009, 71-73.
} 
(Bohême), poignée de couvercle d'une cruche de la tombe princière de Reinheim (Sarre, Allemagne) ornée d'un quadrupède à tête humaine, agrafe de ceinture de Herrnsheim (Alzey-Worms, Allemagne)... ${ }^{60}$. Les vestiges de l'une d'elles (en cuir avec armature en fil de fer) auraient été identifiés dans la sépulture du Glauberg. Si la puissance et le pouvoir de l'homme étaient matérialisés par cette coiffe végétale, il en est peut-être de même pour les sangliers symbolisés par les trompes gauloises.

"Des têtes sur lesquelles poussent des plantes ont leurs homologues dans le monde méditerranéen, quand il s'agit de divinités ou d'êtres fabuleux, sans que leur signification nous soit tout à fait accessible. (...) on les a toujours considérées comme un attribut divin." ${ }^{\circ 1}$. "Les étranges oreilles à la Mickey Mouse qui encadrent ou surmontent des têtes humaines masculines, dans la petite plastique en bronze aussi bien que sur les sculptures de grande taille des $\mathrm{v}^{\mathrm{e}}$ IV ${ }^{\mathrm{e}}$ s. av. J.-C., ont longtemps déconcerté les spécialistes. Elles sont associées à une héroïsation ou une célébration du personnage et peuvent accompagner des attributs de la guerre et du pouvoir tels que bouclier, épée et collier à pendeloque" ${ }^{62}$.

\section{Datation \\ (Chr. Maniquet)}

Bien que la construction du sanctuaire de Tintignac ne remonte guère avant le milieu du $\mathrm{II}^{\mathrm{e}} \mathrm{s}$. a.C., certains éléments découverts dans le dépôt paraissent plus anciens, comme semblent l'indiquer la stylistique ou la technologie de certains casques ${ }^{63}$. Létude des armes, dont la typologie est mieux cernée, permet de les attribuer à une période contemporaine de la mise en place du lieu de culte. Aucun objet ne peut être de façon certaine, en l'absence de signe caractéristique, associé à une période plus récente. De nombreuses pièces restent difficiles à placer au sein de typologies attestées, du fait de leur rareté. On sait simplement, d'après la stratigraphie $^{64}$ que leur enfouissement a été réalisé au plus tard au cours de la seconde moitié du ${ }^{\text {er }}$ s. a.C. La

60- Frey 2008a, 64-71 ; Guggisberg 2009, 47

61. Frey 2008b, 61.

62- Guggisberg 2009, 47.

63. Maniquet 2009c.

64. Maniquet 2009b. datation des objets du dépôt de Tintignac reste donc difficile à définir. Ils appartiennent à une longue période pouvant aller de la fin de La Tène B2 jusqu'à La Tène $\mathrm{C} 2 / \mathrm{D} 1{ }^{65}$, voire au-delà.

Le carnyx existe au moins depuis le début du III $^{\mathrm{e}}$ s. a.C. ; il apparaît pour la première fois sur le revers d'une monnaie grecque où une femme personnifiant l'Étolie écrase un carnyx sous ses pieds. Cette frappe monétaire devait commémorer la résistance des Étoliens face à l'invasion des Gaulois de Brennus en 279 avant notre ère ${ }^{66}$. Il apparaît ensuite sur un denier frappé vers 206/200 a.C., à la période où Rome s'efforce de soumettre les Gaulois implantés en Cisalpine, sur lequel deux cavaliers foulent aux pieds de leur monture un bouclier et un carnyx ${ }^{67}$.

Le collier dont sont dotées les hures de sangliers en bronze coulé, décoré d'un motif abstrait, ou encore les anneaux moulés enserrant les tôles martelées composant la partie supérieure des autres trompes de Tintignac, rappellent certains anneaux de chevilles massifs à oves creux, comme celui de Méry-sur-Seine ${ }^{68}$, généralement datés du $\mathrm{III}^{\mathrm{e}}$ s. a.C. Les pavillons en bronze moulés pourraient avoir fonctionné avec des tubes également moulés découverts épars dans le dépôt et pourraient représenter une autre génération (plus ancienne?) de trompette. Ceci n'implique cependant pas forcément une datation aussi haute pour les autres trompes en tôles de bronze.

Sans plus de preuve, cette trompette pourrait aussi bien être contemporaine des objets les plus anciens, soit le début du $\mathrm{III}^{\mathrm{e}}$ s. a.C., que juste antérieure à la constitution du dépôt, dans la seconde moitié du ir s. a.C.

\section{Perspectives \\ (Chr. Maniquet)}

L'ambition désormais est de restituer un carnyx au plus près de la réalité, en utilisant, dans la mesure du possible, les mêmes alliages, les mêmes techniques de fabrication, les mêmes assemblages. Le but ultime n'est pas seulement de reproduire l'objet

65. Maniquet 2008, 315-317.

66- Jenkins $1972 n^{\circ} 546$; Vendries 1993, 28.

67- Crawford 1974, vol. I, n ${ }^{\circ} 128,207$; vol. II, pl. XXIII, n 10.

68- Hautenauve 2001, 152. 
dans son intégralité, mais également d'étudier les sons qu'il pouvait émettre. À ce jour, aucune reconstitution n'est dotée d'une embouchure droite ou d'oreilles. Elles s'inspirent pour la plupart de reliefs de monuments romains et de représentations monétaires n'apportant aucune information sur les matériaux employés.

Devant l'absence de découverte d'embouchure de carnyx jusqu'en 2004, le faible nombre de représentations de ces dernières sur des monnaies (toutes droites) et le caractère trompeur de la seule illustration de l'instrument en fonctionnement (le bassin de Gundestrup), l'embouchure était considérée courbe et a été presque systématiquement restituée comme telle. De façon étonnante, les plus anciennes reconstitutions, telle celle construite vers la fin du $\mathrm{XIX}^{\mathrm{e}}$ s. accompagnant un mannequin gaulois du Musée de l'Armée à Paris ou encore celle réalisée pour le Musée de Mayence vers 1912-1913, ont été dotées d'une embouchure droite, plutôt réaliste ${ }^{69}$. Les reconstitutions plus récentes (celle fabriquée à partir des vestiges de Deskford en 1999, et celle du musée de Bibracte en 1996) sont toutes pourvues d'une embouchure courbe. En réalité cette erreur est assez récente : "Le choix d'une embouchure droite est incompatible avec le jeu en position verticale mais il est vrai que les monnaies, les reliefs romains de l'arc d'Orange ${ }^{70}$ ou de la colonne trajane ne montrent jamais l'embouchure, masquée par un amoncellement d'armes diverses. Il faut peut-être considérer que l'embouchure était mobile. Cette erreur dans le profil de l'embouchure a été heureusement rectifiée par Fraser Hunter lors de sa reconstitution $^{71}$. En 2009, Fraser Hunter écrit : "one key point to emerge is that, contrary to most reconstructions (including one the author was involved in !), the carnyx had a mouthpiece at the end of a straight tube, with no curve to make it easier for the player" 72 . La reconstitution du carnyx de Tintignac permettra, avec l'aide de musiciens et de musicologues, de se faire une idée précise des sons que pouvaient en tirer les Gaulois.

\section{LE CASQUE-OISEAU}

\section{Description \\ (Th. Lejars)}

Longueur max. $535 \mathrm{~mm}$; largeur max. $158 \mathrm{~mm}$; hauteur max. $430 \mathrm{~mm}$.

Timbre : longueur $225 \mathrm{~mm}$; largeur $158 \mathrm{~mm}$; hauteur $143 \mathrm{~mm}$; circonférence interne base $610 \mathrm{~mm}$; hauteur base $25 \mathrm{~mm}$.

Tête et cou : Longueur (développée externe) $990 \mathrm{~mm}$; longueur tête $103 \mathrm{~mm}$; largeur tête $32 \mathrm{~mm}$ (38 mm avec les yeux, un seul est conservé).

Appendice caudal : longueur 190 mm ; largeur extrémité $50 \mathrm{~mm}$.

Poids $1400 \mathrm{~g}$

Conservation : bon état. Patine verte à vert foncé, localement bleue à noire. La surface est granuleuse et mieux préservée sur la face qui reposait sur le sol.

Le casque complet, en bronze, en forme d'oiseau (fig. 45 et 46) reposait sur son flanc gauche, directement sur le fond. Le poids des sédiments et des objets, en bronze et en fer, qui le recouvraient, a provoqué un léger écrasement des parties latérales. L'objet, très légèrement déformé, présente, sur un côté, des traces de coups (fig. 47). Les marques verticales et sensiblement parallèles, qui ont touché plus particulièrement le timbre et le col, n'ont pas provoqué de déchirure. On note en revanche un déchirement de la base, sur l'arrière, et une perforation de la face antérieure gauche provoquée par une armature nervurée, probablement un fer de lance (fig. 48). Le refoulement du métal sur les côtés paraît avoir été provoqué par le retrait du fer. Les impacts relevés sur la tôle, relativement superficiels, n'ont eu aucune incidence sur la physionomie générale de l'objet, ce qui peut étonner du fait de sa taille, de la faible épaisseur des tôles et de sa morphologie très élancée. Compte tenu du contexte de dépôt, cette bonne tenue de la structure métallique implique un colmatage rapide du fond de la fosse et de l'intérieur du casque.

69. Vendries 1999, fig. 10 et 12, 384-385.

70. Amy et al. 1962.

71- Vendries 1999, 386-387.

72. Hunter 2009b, 244. 


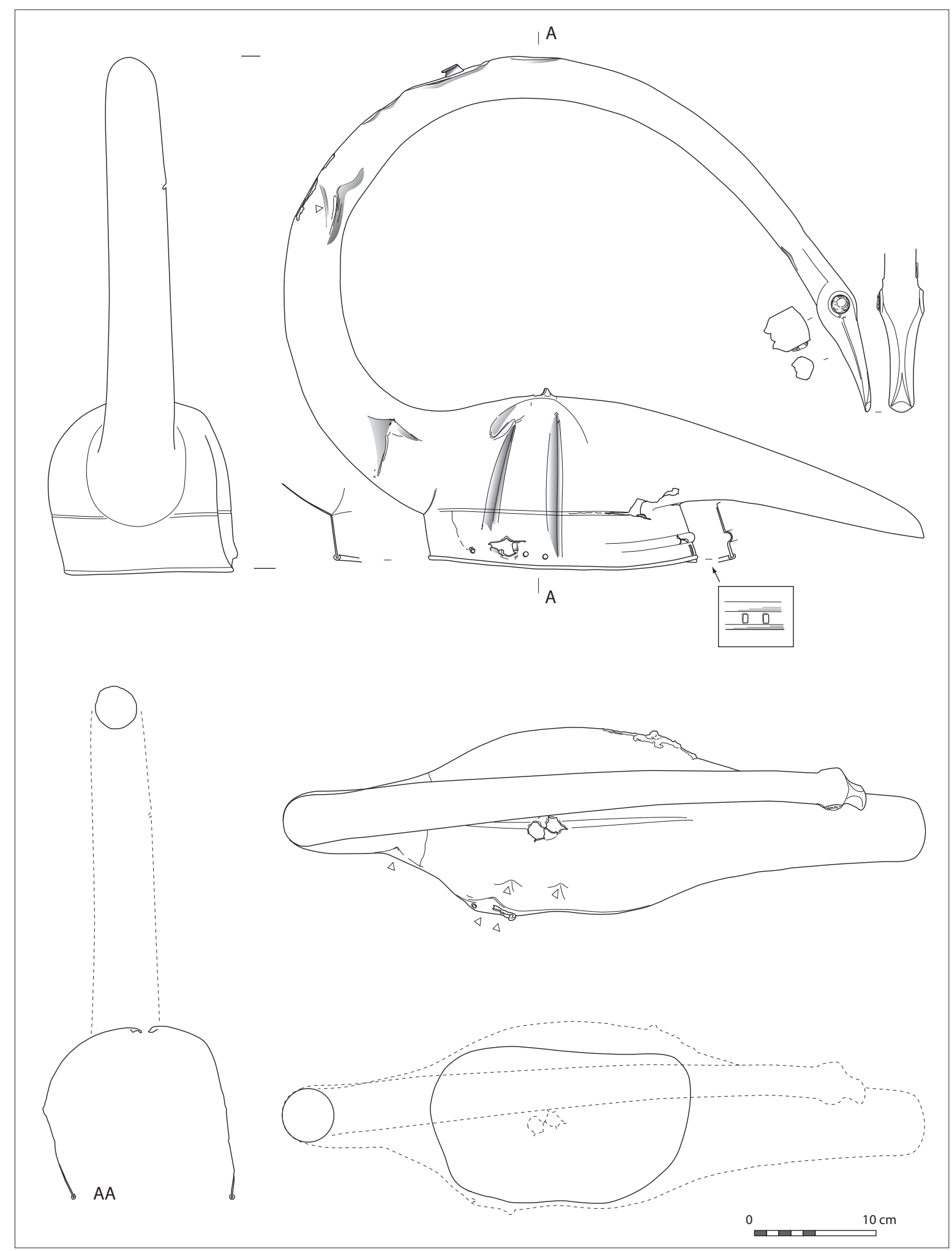

Fig. 45. Casque-oiseau de Tintignac (Th. Lejars, CNRS). 


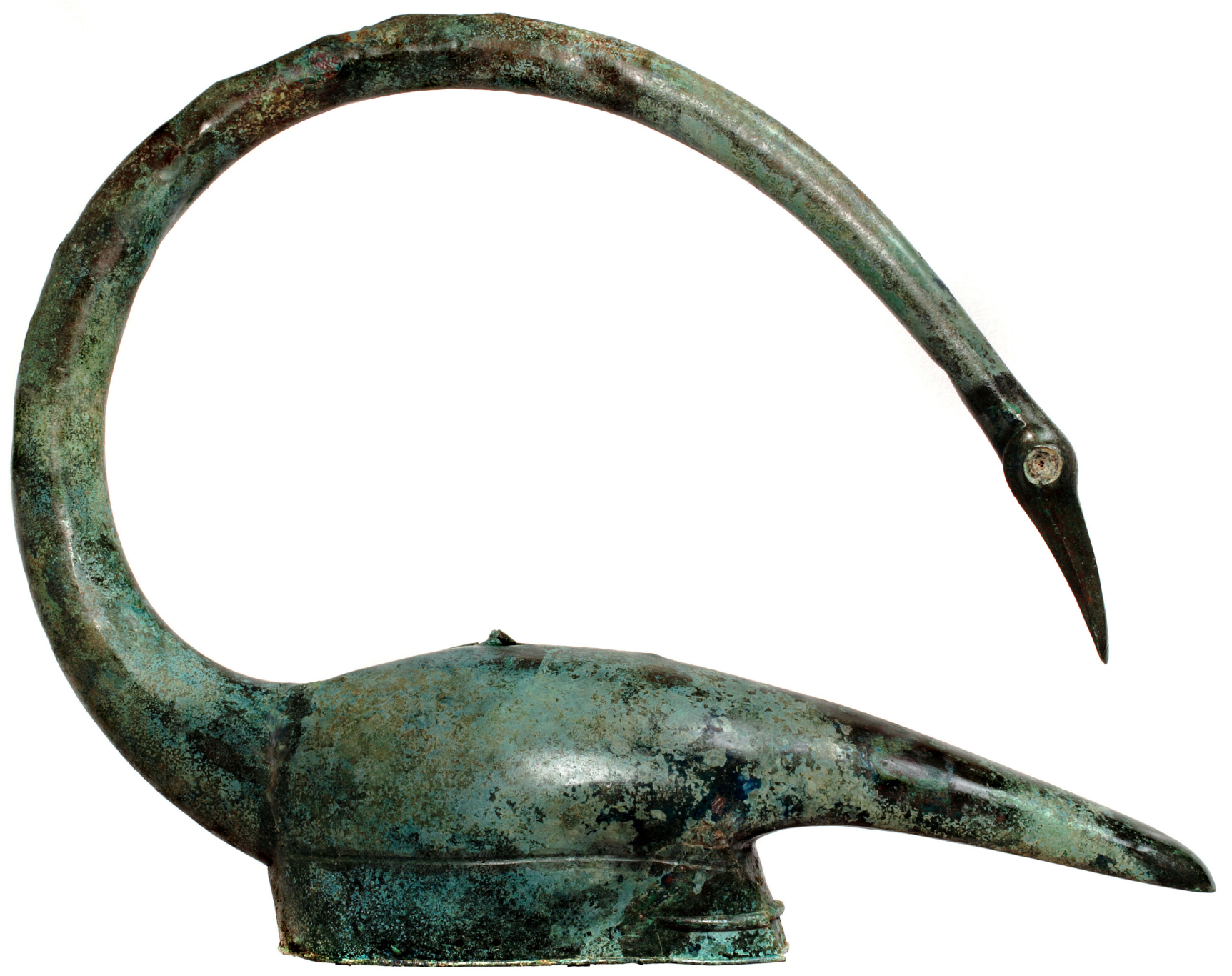

Fig. 46. Casque-oiseau, face gauche (cl. P. Ernaux, INRAP).

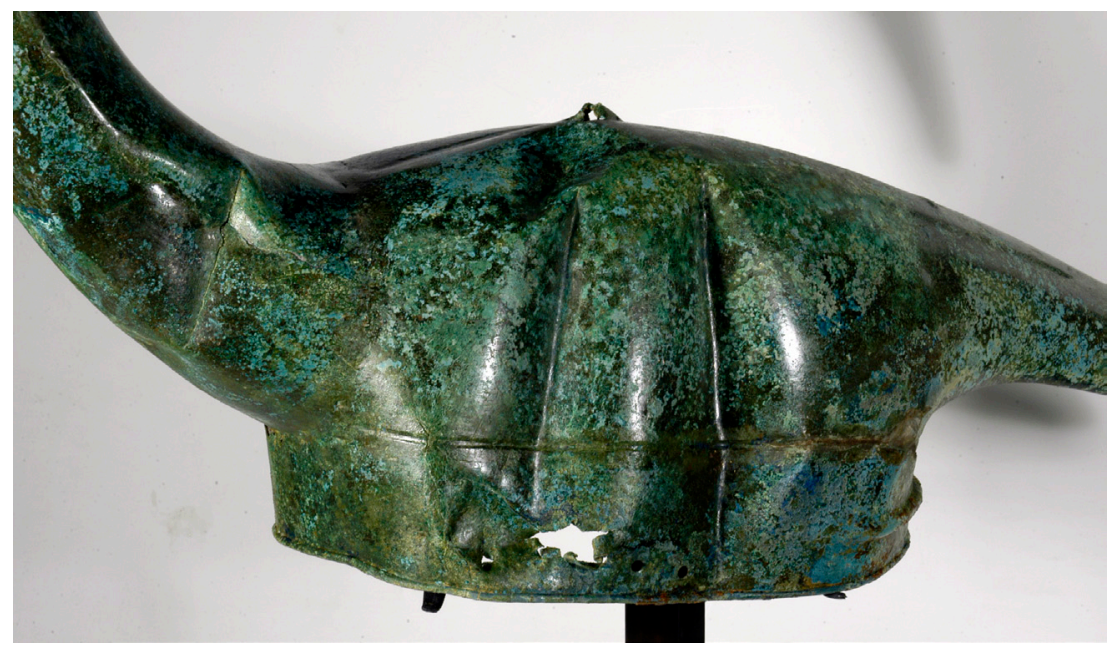

Fig. 47. Flanc droit du casque

marqué de coups linéaires

(cl. B. Armbruster, CNRS). 


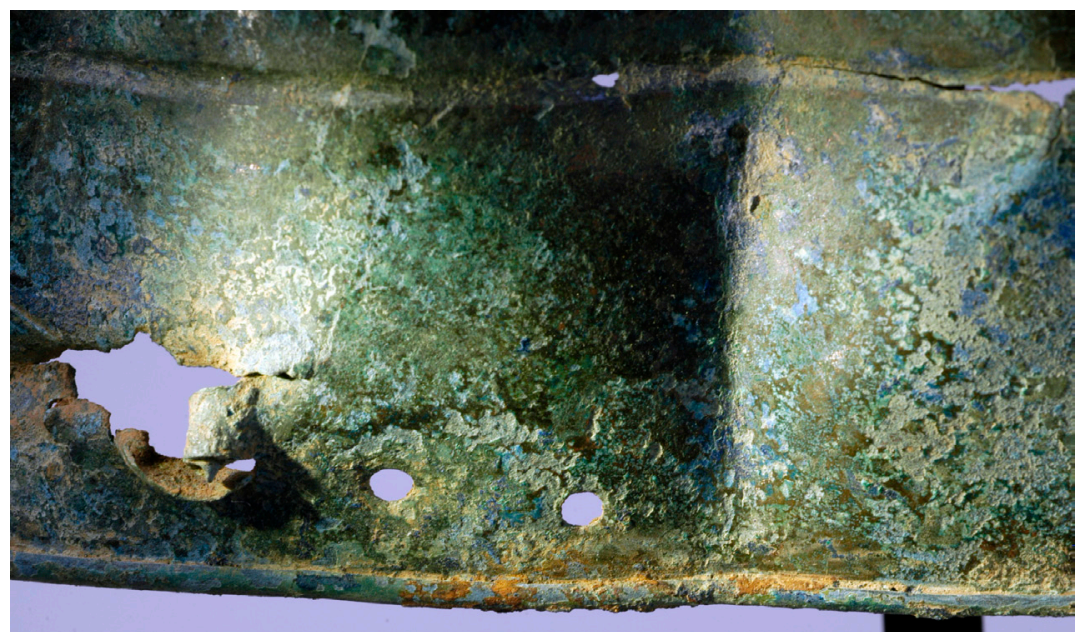

Fig. 48. Perforations à la base du flanc droit du casque (cl. B. Armbruster, CNRS).

Le casque est constitué de onze tôles soudées et brasées. Le timbre paraît avoir été réalisé par martelage à partir d'une ébauche coulée, tandis que la tête d'oiseau, plus massive, a été obtenue par la technique de la cire perdue (cf. infra, Études métallurgiques et technologiques). Il n'y a aucune trace de rivet et le polissage final a éliminé les marques des soudures qui ne sont plus visibles qu'à la radiographie $\mathrm{X}$. Le casque se compose de trois parties : le timbre (le corps de l'oiseau), l'excroissance postérieure (l'appendice caudal) et enfin la partie frontale qui se développe en arc de cercle au-dessus et vers l'arrière (le col et la tête). Le timbre est lui-même divisé horizontalement en deux parties distinctes séparées par une rainure plus marquée sur l'arrière et les côtés. Alors que la moitié supérieure, ovoïde, constitue le corps de l'animal proprement dit, la partie inférieure, réa- lisée séparément et nettement différenciée, venait ceindre la tête du porteur. La partie supérieure ne présente aucun trait particulier à l'exception d'une double perforation irrégulière, au sommet (fig. 49). Ces trous ont été percés depuis l'extérieur, tandis que le métal était refoulé vers l'intérieur et la partie centrale exhaussée. La technique d'exécution particulièrement sommaire suggère qu'ils furent réalisés dans un second temps. La position centrale de ce double orifice et son aspect fruste ne sont pas la marque d'une destruction volontaire, mais bien plus probablement d'un "bricolage" destiné à la réalisation d'une bélière, pour fixer un ornement en matériau organique ou servir de support pour faciliter la suspension du casque. La base, légèrement plus développée sur l'arrière, a la forme d'un cylindre ovale. Le tiers postérieur se signale en outre par un large

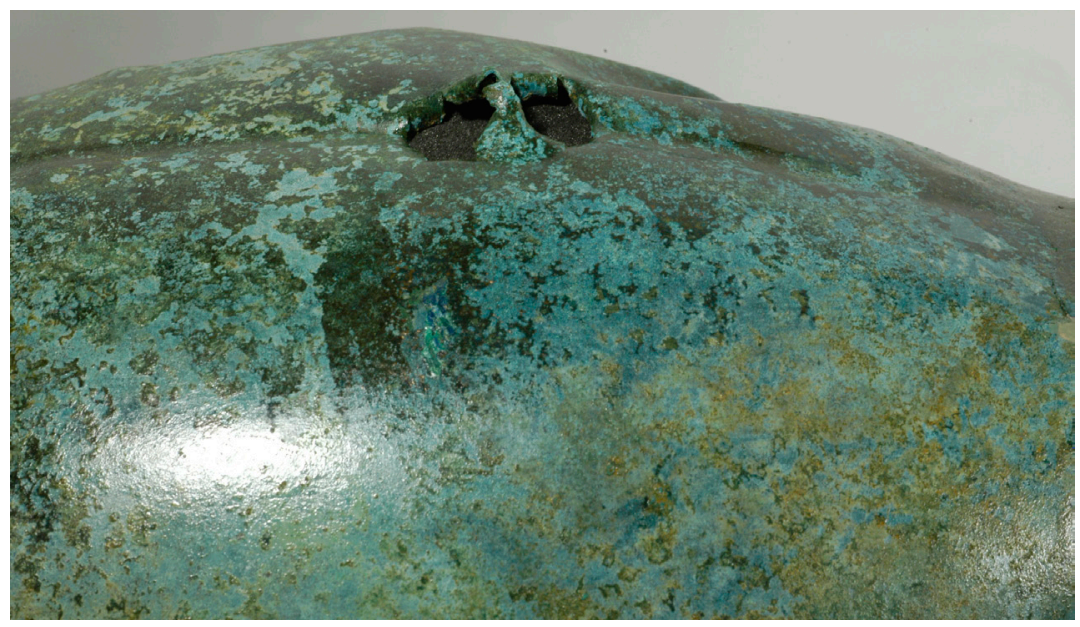

Fig. 49. Perforations au sommet du timbre (cl. B. Armbruster, CNRS). 
bourrelet horizontal qui s'estompe progressivement sur les côtés. Il avait également pour fonction de renforcer la structure métallique. La bordure, marquée par une légère surépaisseur (env. $2 \mathrm{~mm}$ ) est renforcée par l'adjonction d'un orle arrondi (3,4 $\mathrm{mm}$ de large pour 4,3 mm de hauteur) selon un procédé fréquemment utilisé pour les casques en $\mathrm{fer}^{73}$. En l'absence de tout système de fixation, l'hypothèse d'un couvrenuque réalisé séparément n'est pas démontrée. La présence du bourrelet qui signale de la même façon de nombreux couvre-nuques rapportés (c'est le cas notamment des autres casques de Tintignac), suffit pour désigner la partie postérieure et la manière dont se présentait l'objet. Deux trous réguliers de 4 $\mathrm{mm}$ de diamètre et espacés de 13, ont été aménagés sur les côtés, légèrement décalés vers l'avant (fig. 50). Là encore, rien ne permet d'affirmer l'existence de paragnathides. Il est plus probable de voir dans ces orifices les points de fixations destinés à recevoir les éléments en cuir de la jugulaire (ou de l'articulation en cuir d'éventuels couvre-joues). Les deux découpes rectangulaires de $7 \mathrm{~mm}$ de hauteur sur 4 de large, placées à l'arrière dans l'axe, et espacées de $13 \mathrm{~mm}$, paraissent d'origine en dépit de leur irrégularité (fig. 51). Leur raison d'être demeure incertaine. On pro- posera de voir là, à titre d'hypothèse, et par analogie avec les anneaux postérieurs des casques de type Montefortino, un élément lié au port du casque qui offrait ainsi une meilleure stabilité : les extrémités de la lanière, passées par les deux trous rectangulaires, entouraient le cou et venaient croiser sous le menton pour rejoindre l'attache latérale opposée comme le montre un buste de Pyrrhos provenant d'Herculanum (fig. 52) ${ }^{74}$. On peut aussi y voir, plus simplement, la fixation pour un panache ou toutes autres sortes d'ornements placés à l'arrière.

La partie postérieure, correspondant à la queue, est constituée d'un appendice triangulaire tronqué, incliné vers le bas, long d'une vingtaine de centimètres. La transition avec le timbre est parfaitement continue. Le raccord avec la partie tubulaire antérieure se signale en revanche par un changement de volume. Le tube, de plus fort diamètre à la base, s'amincit progressivement et dessine au-dessus du timbre une large boucle. Une tête d'oiseau en marque l'extrémité. Le bec, long d'environ $8 \mathrm{~cm}$ et arqué vers le bas, pointe en direction de la queue. Une rainure également arquée souligne de la même façon la commissure du bec. Vu de dessus, le bec

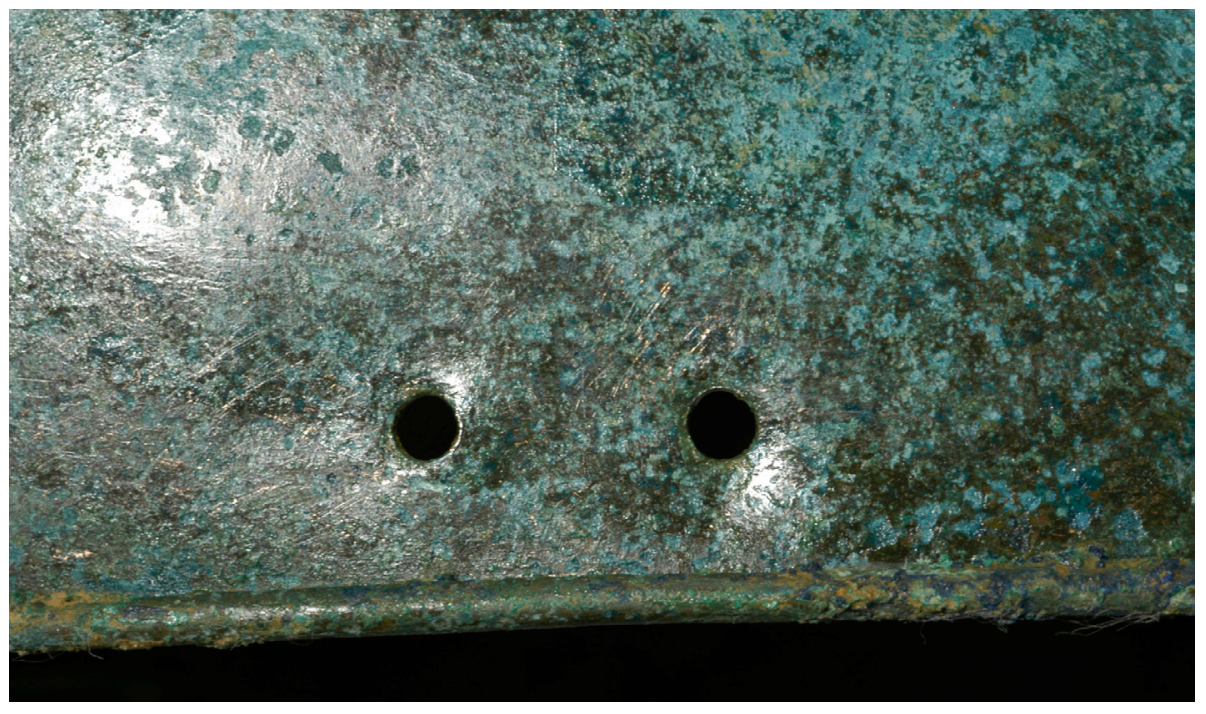

Fig. 50. Petits trous réguliers à la base du flanc gauche du casque (cl. B. Armbruster, CNRS). 


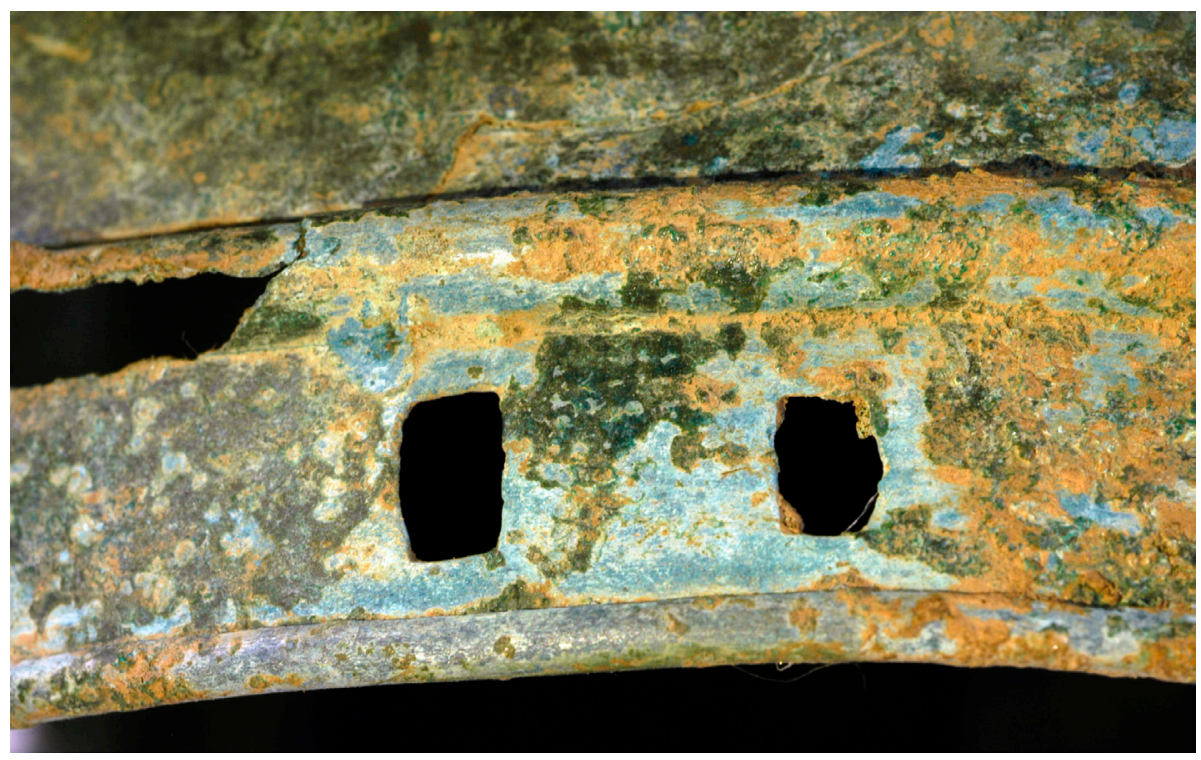

Fig. 51. Petits trous rectangulaire à l'arrière, à la base du timbre (cl. B. Armbruster, CNRS).
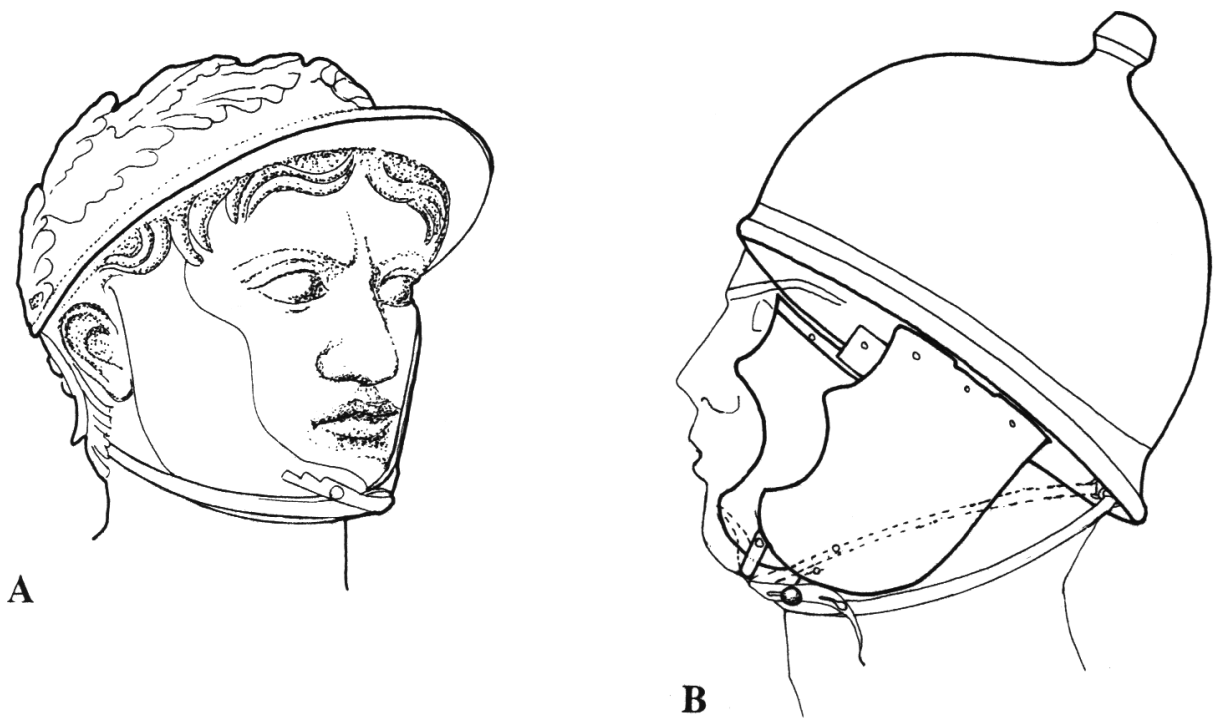

Fig. 52. A. Buste en marbre de Pyrrhos, copie romaine d'un original hellénistique, prov. Herculanum (Naples, Musée Archéologique National, d'après Ducrey 1985) ;

B. Casque à bouton sommital de type Montefortino. Reconstitution du système d'attache avec liens croisés autour du cou (d'après le buste de Pyrrhos). 


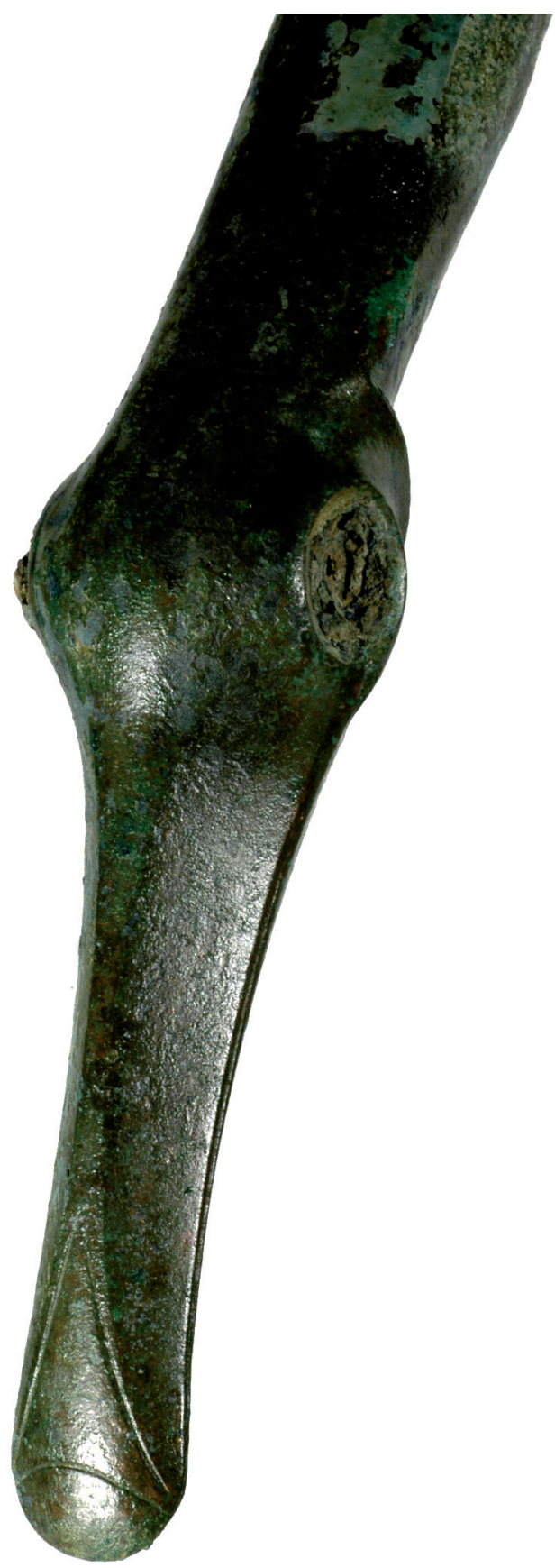

Fig. 53. Détail de la tête de l'oiseau (cl. B. Armbruster, CNRS). n'apparaît plus effilé mais épaté avec le bout arrondi à la manière d'un palmipède (fig. 53). Un motif triangulaire allongé, à base concave, souligne l'épanouissement de l'extrémité. La base concave forme enfin avec l'extrémité du bec un motif parfaitement lenticulaire. Le rendu géométrique, et non réaliste, est accentué par le traitement en méplats du bec et la fluidité des formes. Le passage graduel du bec à la tête est renforcé par le tracé continu du rebord inférieur du bec qui s'incurve pour dessiner l'arrière de la tête. À cela s'ajoute la forme arrondie de la tête et la profonde gorge anguleuse qui se développe au-dessous, depuis l'arrière de la tête jusqu'à la pointe du bec (fig. 54). La tête est, quant à elle, légèrement bombée sur le dessus et franchement anguleuse dans sa partie inférieure. Les côtés, traités en méplats, font ressortir au centre les grands yeux circulaires polychromes d'une quinzaine de millimètres de diamètre formés d'un disque noir en lignite, entouré de blanc et cerné d'une couronne dorée ${ }^{75}$ (fig. 55).

\section{Étude technologique (B. Armbruster)}

Le casque est un corps creux en tôle de bronze composé de plusieurs éléments, dont la majorité des parties est martelée (fig. 56). Les tôles le constituant sont d'épaisseurs faibles, de 0,5 à $2 \mathrm{~mm}$. Comme pour le carnyx, les fines tôles jouaient un rôle important pour alléger le poids du casque (environ $1,5 \mathrm{~kg}$ ) malgré le volume et la taille de l'objet. Contrairement au carnyx, le casque porte peu d'éléments de décor en relief, quelques traits sur la tête, autour du bec et des yeux. Des sillons discrets ciselés, l'un entre les parties supérieure et inférieure du casque, l'autre sur le dos du cygne, sont des éléments structurants et renforçant l'ensemble.

Le casque est actuellement constitué de dix éléments réalisés séparément puis assemblés. Un seul élément, la tête creuse avec le bec, est uniquement issu de la coulée. C'est donc le seul élément, qui n'a pas subi une déformation plastique. Les éléments en tôle sont le cou, constitué de six éléments, la partie

75- Voir la statue en bronze de Bouray qui se signale par des yeux en verre bleu et blanc; Megaw \& Megaw 1989, 156. La question des yeux et du matériau utilisé se pose de la même façon pour les enseignes et carnyx provenant du dépôt. 

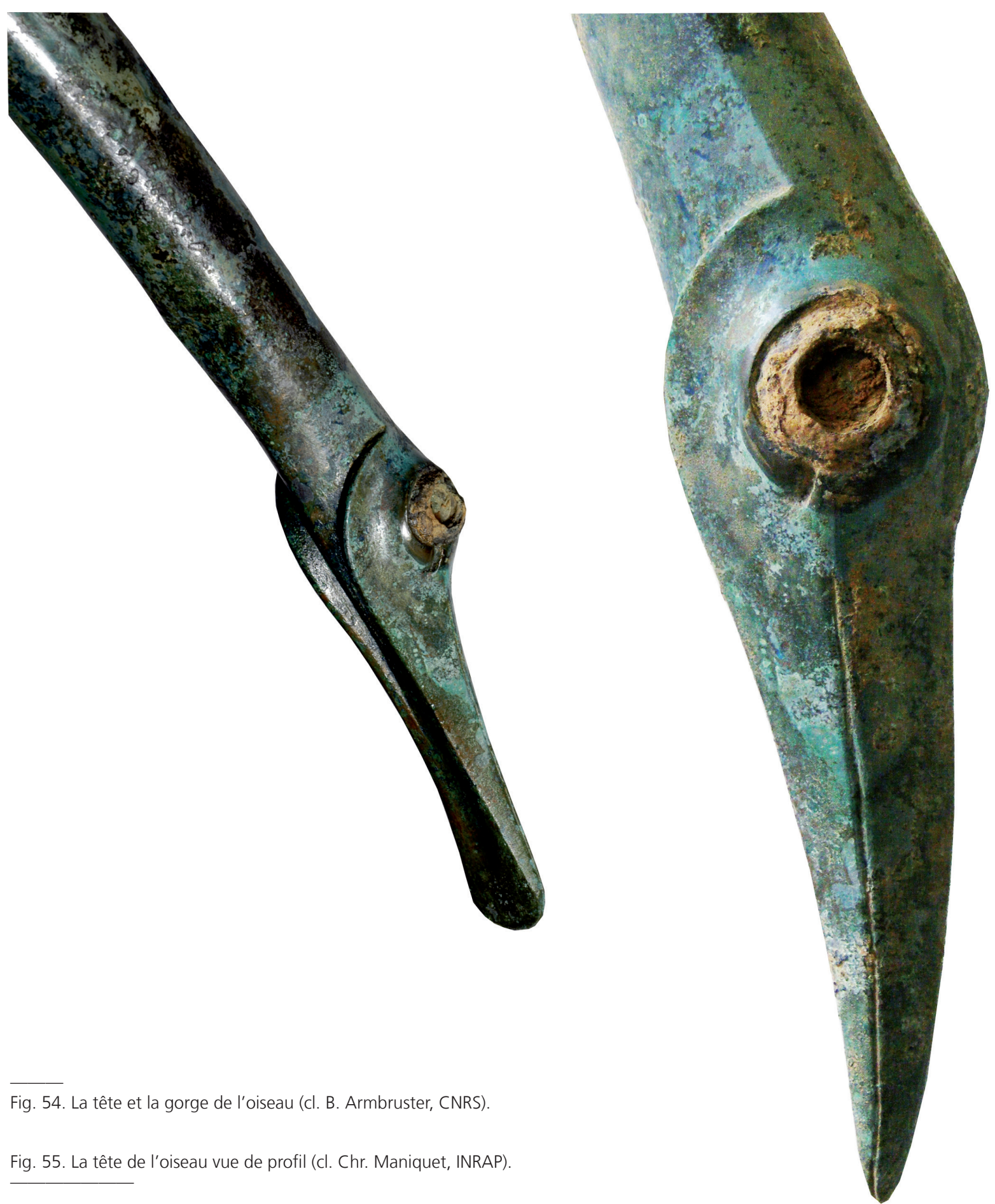

Fig. 55. La tête de l'oiseau vue de profil (cl. Chr. Maniquet, INRAP). 
supérieure du casque ("le timbre"), incluant la partie supérieure de la queue, la partie inférieure en forme d'un ovale "extrudé" du casque, incluant la partie inférieure de la queue, et une bande mince sur le bord. Cette bande longue et fine, repliée en forme de tube ouvert sur sa longueur, a été rapportée au bord (fig. 57), qu'elle sert à renforcer et à l'arrondir, et ainsi éviter de probables blessures. On peut supposer la disparition de certains éléments comme le couvre nuque et les paragnathides, fixés à l'aide de pièces d'articulation.
Les techniques et la chaîne opératoire de la fabrication du casque

L'ordre technique de la chaîne opératoire du casque est comparable à celui du carnyx : l'artisan débute son projet avec les éléments de fonderie. Suivent les étapes de martelage et de ciselure interrompus de recuits, puis l'assemblage par brasure, la finition par polissage et finalement l'incrustation.

La tête d'oiseau est coulée à la cire perdue (fig. 53 à 55). Creuse, elle a d'abord été modelée en cire sur un noyau en argile, puis coulée. Le modèle en cire

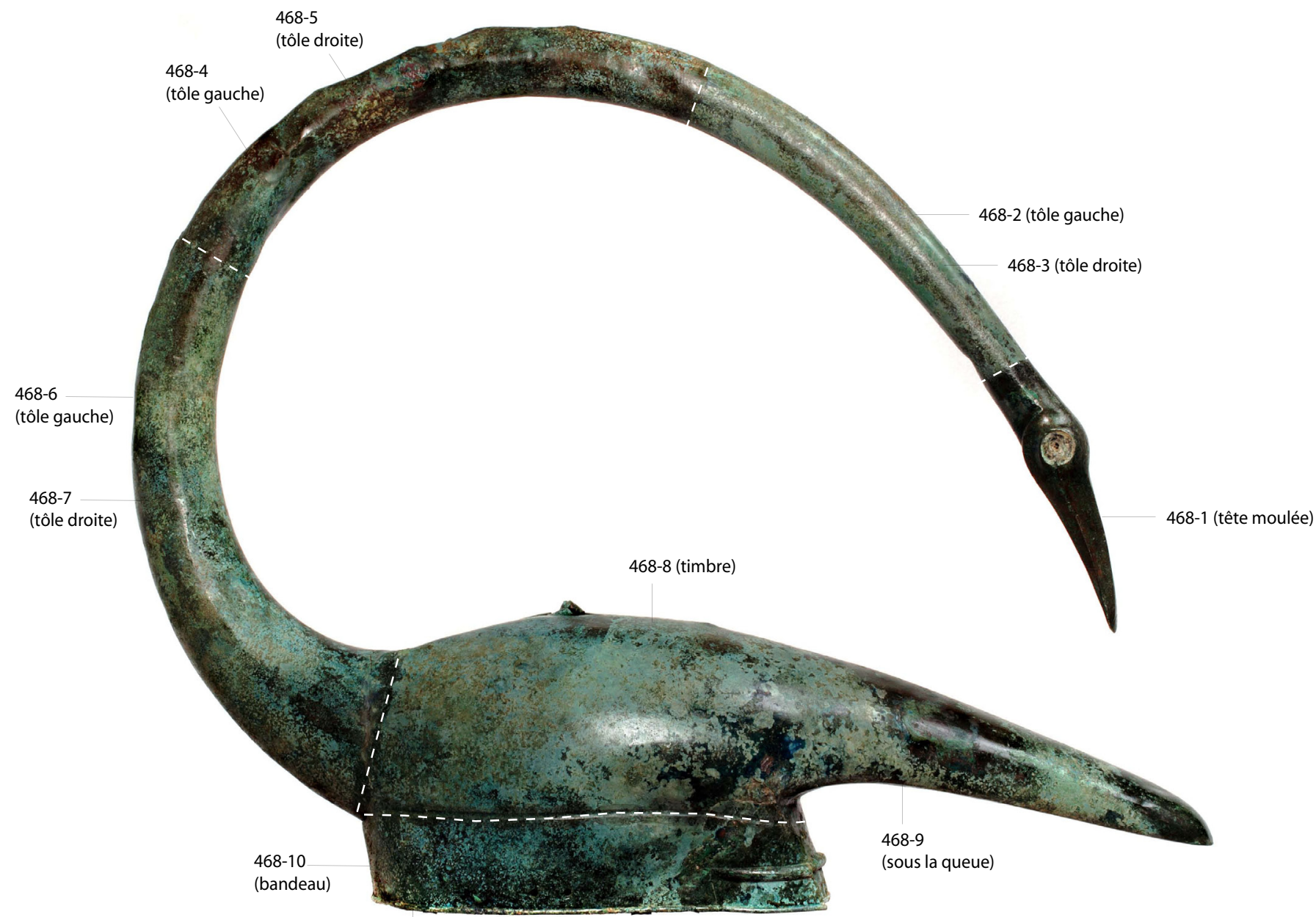

468-11 (orle)

Fig. 56. Le casque et ses parties constitutives (cl. P. Ernaux, INRAP). 


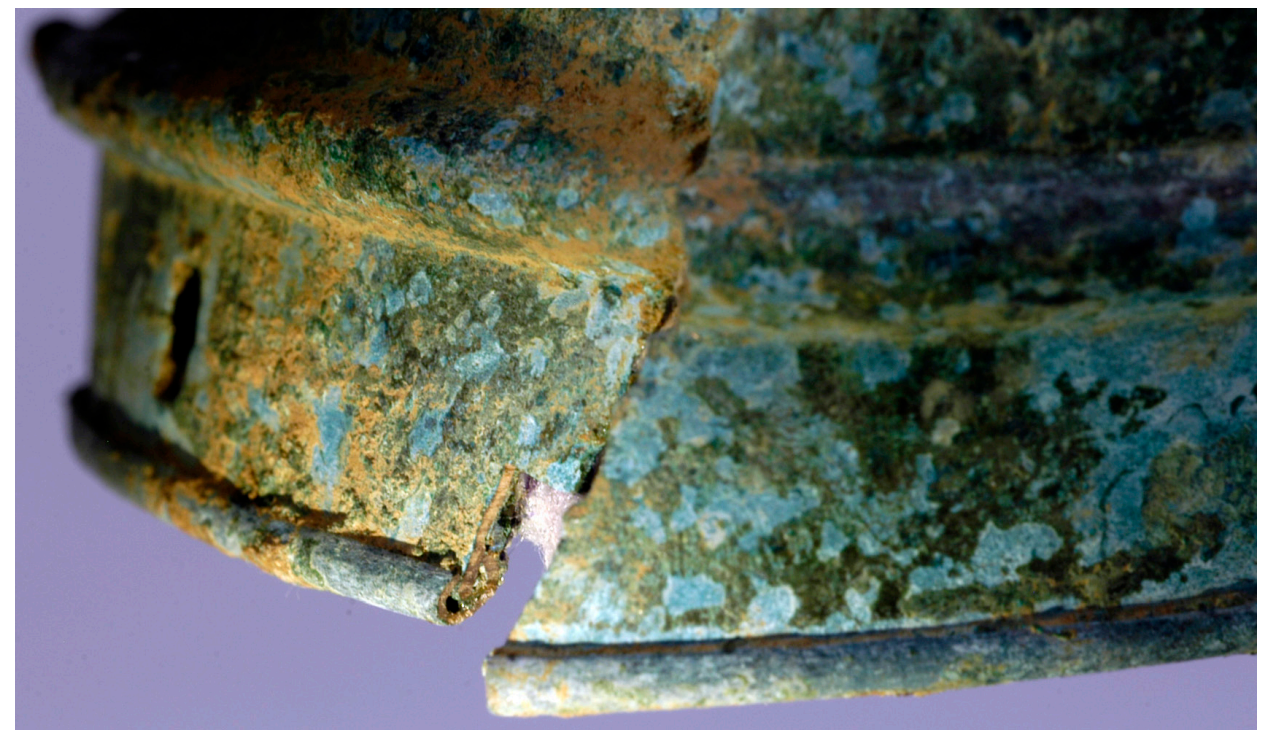

Fig. 57. Orle rapporté au bord du casque (cl. B. Armbruster, CNRS).

portait déjà tous les détails. Après la coulée et la finition de la surface par abrasion, l'artisan a retracé les traits fins par ciselure en utilisant un ciselet plat. Le corps, la queue et le cou sont des parties mises en forme par martelage à partir de produits préliminaires coulés, comme des lingots ou des ébauches. La partie du corps est composée d'une calotte ovale comportant une partie de la queue, et d'un bandeau rajouté attenant peut-être lui aussi à la base de la queue. Un trait ciselé cache cette jonction des deux parties du corps (fig. 58). Le cou est constitué de trois segments formés de deux coques chacun. L'assemblage par brasure des coques a été réalisé latéralement avant de les unir dans la longueur. Ce mode d'opération particulier indique qu'il fallait fabriquer six éléments en tôle de différentes courbures bien ajustées. Le dinandier choisissait cette manière de procéder pour répondre aux contraintes mécaniques engendrées par le poids du cou soutenu sur un seul côté. La mise en forme du casque requiert un travail de précision et d'extrême finesse pour réaliser ce corps creux composé de deux volumes distincts. Le long cou creux et courbe avec la tête orientée vers la queue doit résister à d'importantes contraintes mécaniques dues à sa longueur et son poids, et notamment celui de la tête coulée sans aucun appui. Contrairement au carnyx, les différentes parties ont été uniquement assemblées par brasure, à l'exception du petit bandeau replié et rapporté mécaniquement par emboîtage au rebord (fig. 57).

Les différentes parties constituant le casque ont été très soigneusement liées par brasure, probablement de l'étain, les rendant difficiles à distinguer à première vue sur les surfaces corrodées avant restauration. Dans ce contexte, les clichés radiographiques facilitent la distinction des différents composants. Des taches grises, stigmates des brasures, sont apparues clairement pendant la restauration sur la surface extérieure (fig. 58 et 59). Des restes de la matière de brasure sont aussi présents à l'intérieur, notamment à la jonction entre le cou et le corps.

Plusieurs perforations doubles sont situées sur le bandeau servant de point de fixation d'éléments aujourd'hui manquants (fig. 50 et 51). Les trous circulaires sur les deux côtés au niveau des oreilles du porteur du casque ont été obtenus en perçant avec un foret. Les deux orifices rectangulaires côté nuque ont été découpés à l'aide un ciselet. Au sommet de la calotte du casque, on trouve une double perforation avec des ouvertures, effectuées en forçant une pointe conique de l'extérieur vers l'intérieur (fig. 60). Après 


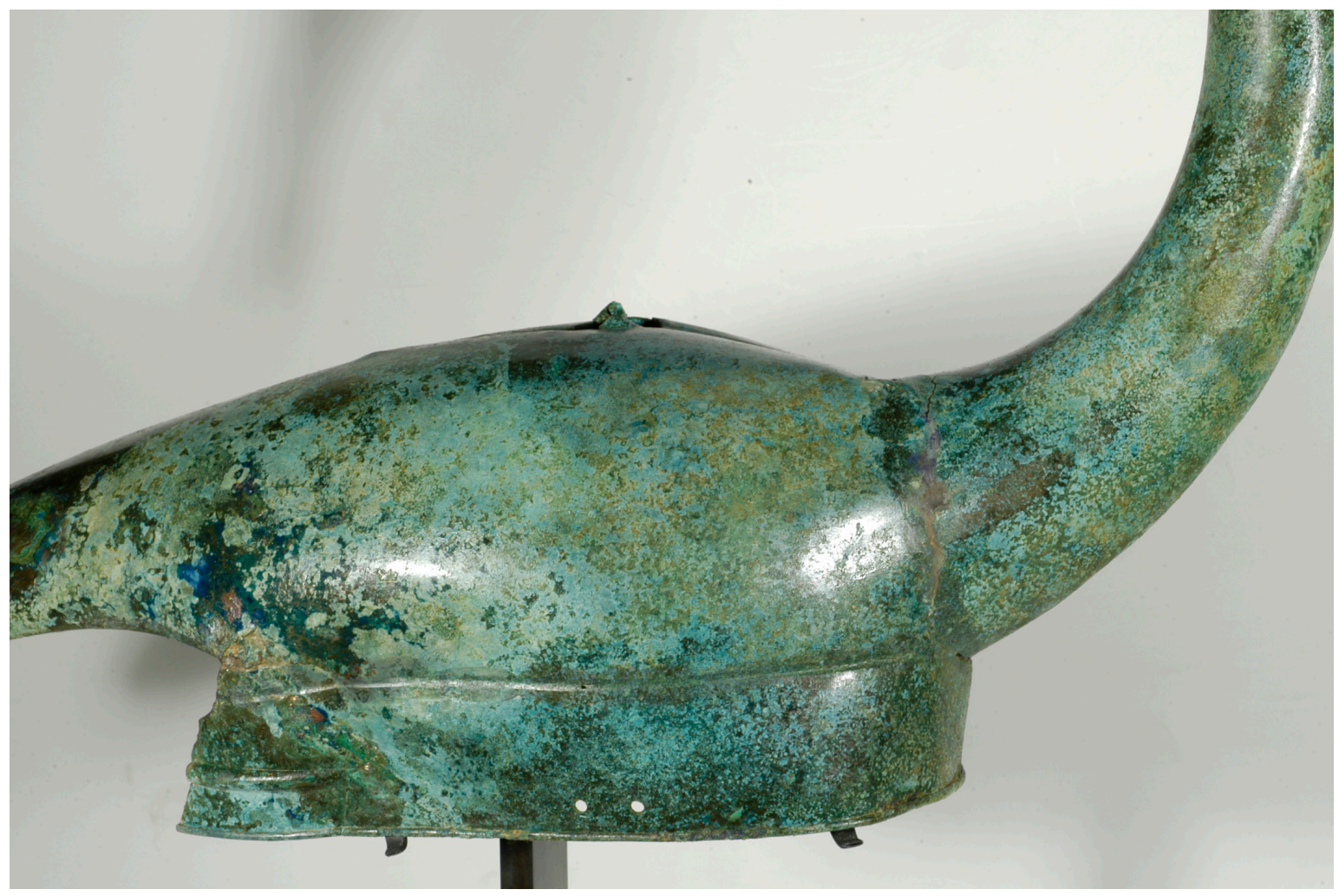

Fig. 58. Traces de brasage sur le casque, à la base du cou (cl. B. Armbruster, CNRS).

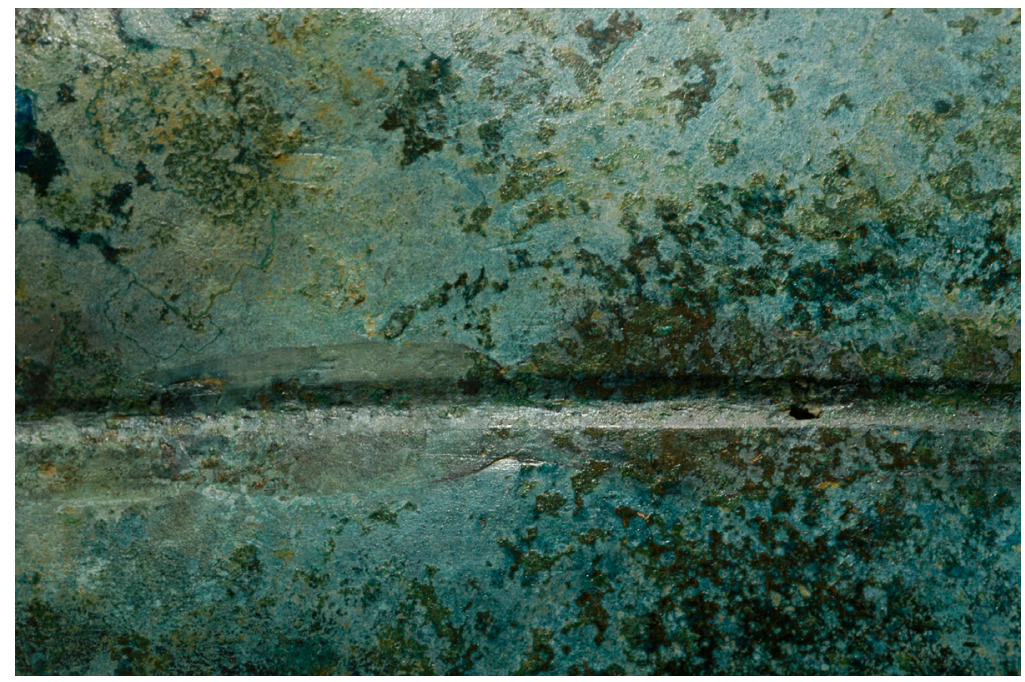

Fig. 59. Détail des taches grises, stigmates de la brasure (cl. B. Armbruster, CNRS). 
plusieurs opérations de brasure, l'ensemble a été soigneusement travaillé avec des matières abrasives. Cette finition qui se termine avec un polissage a rendu la surface complètement lisse et effacé les brasures. Il s'agit de jointures de précision solides et adaptées à la fonction et à la forme particulière du casque.

La technique de l'incrustation a été utilisée pour les yeux : un cercle blanc en étain non allié et une partie centrale noire en lignite (fig. 61) probablement fixée par un adhésif, comme pour le carnyx.

Le riche répertoire des artisans gaulois qui ont mis en œuvre le carnyx et le casque de Tintignac comprenait les techniques suivantes : la coulée en lingotière, la coulée à la cire perdue, la fabrication de tôles par martelage à partir de lingots ou ébauches, la mise en forme par martelage d'objets creux en tôle, la ciselure de reliefs et de décors, le découpage des contours et des parties ajourées, le cintrage, l'emboîtage, le cerclage, la brasure, l'incrustation, peut-être l'étamage et le polissage. La conception complexe des deux objets ainsi que les techniques appliquées dans le travail du bronze et de l'incrustation des yeux sont comparables, malgré leurs formes et fonctions distinctes. Cette convergence sur le plan technique permet d'émettre l'hypothèse d'un probable atelier de bronzier commun. Ces deux objets complexes du dépôt de Tintignac sont d'excellente qualité de fabrication et ils témoignent, par leurs remarquables aspects esthétiques et symboliques, d'un savoir-faire technique de très haut niveau. Ils attes-
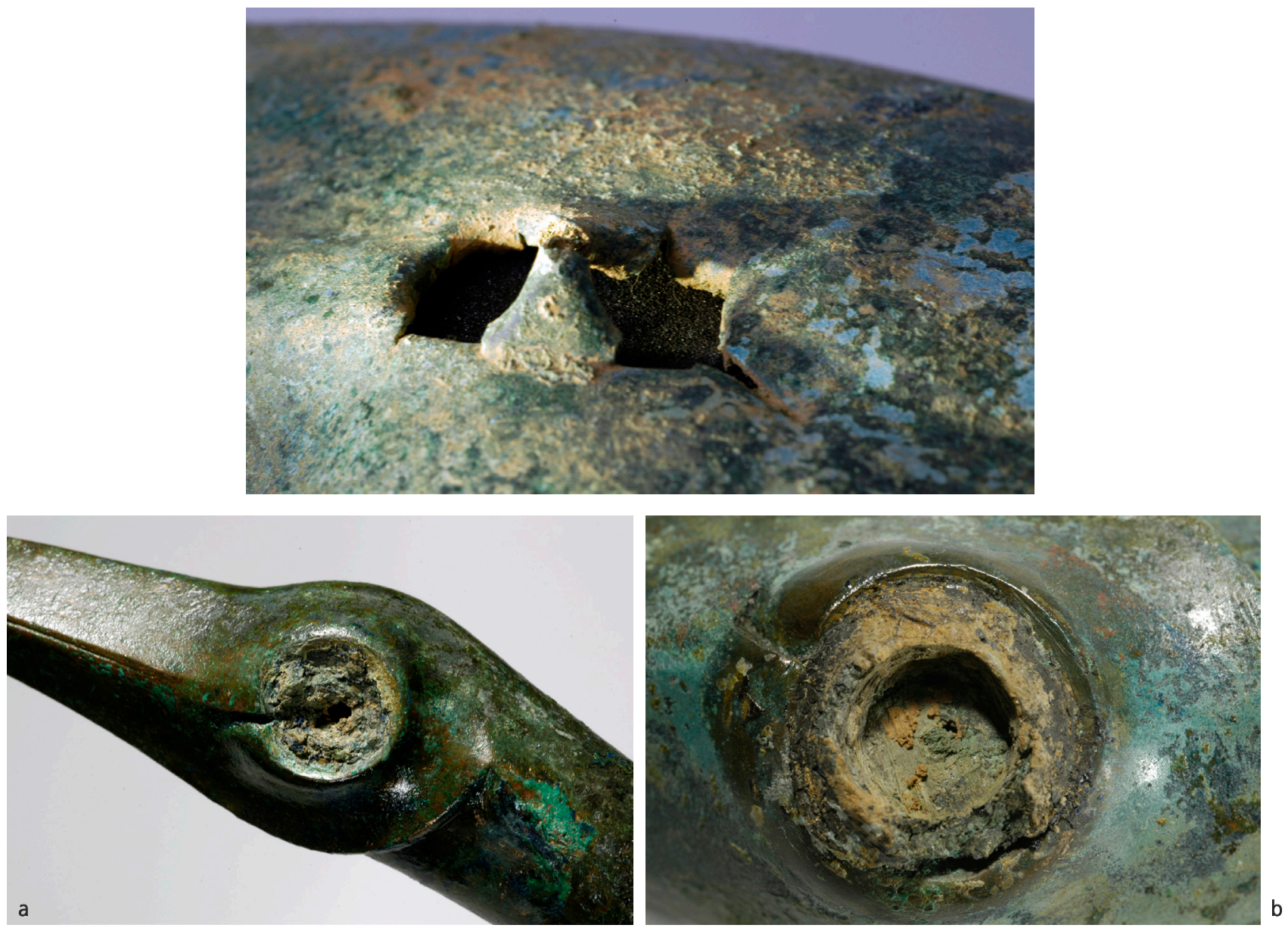

Fig. 60. Double perforation située sur le timbre (cl. B. Armbruster, CNRS).

Fig. 61 a. Détail de l'œil gauche de l'oiseau ; b. Incrustation de l'œil droit (cl. B. Armbruster, CNRS). 
tent la capacité des artisans gaulois de concevoir de grandes ouvres complexes de chaudronnerie, aussi bien par le "design" que par la technologie.

\section{Étude métallurgique}

(M. Pernot, avec la collaboration de Fr. Adamski, S. Campodonico et P. Piccardo)

\section{L'échantillonnage}

La qualité de l'assemblage des différentes pièces est excellente et très bien conservée ; ainsi, il est difficile, à l'examen visuel, d'en estimer le nombre. Les radiographies (fig. 74) ont permis de comprendre que ce casque comporte onze pièces principales qui forment la "structure" de l'objet (fig. 56) ; d'autres pièces "secondaires" participent à la décoration en formant les yeux de l'oiseau. Les assemblages sont, le plus souvent, réalisés par brasage. Compte tenu du bon état de conservation de l'objet, seulement six prélèvements ont été réalisés ${ }^{76}$; deux d'entre eux ont prélevés au scalpel (T2 et T3), car il s'agissait seulement d'identifier le matériau sans chercher à caractériser la microstructure; ils sont tous décrits dans le tableau 2.

\begin{tabular}{|l|l|l|}
\hline \multicolumn{1}{|c|}{ Localisation des prélèvements } & Référence attribuée & \multicolumn{1}{|c|}{$\begin{array}{c}\text { Épaisseur } \\
\text { des tôles }\end{array}$} \\
\hline $\begin{array}{l}\text { Tôle du timbre ; en bas, près } \\
\text { de la jonction avec le bandeau }\end{array}$ & $468-8 /$ TTC 11 & $2 \mathrm{~mm}$ \\
\hline Tôle du bord du casque & $468-10 /$ TTC $12-1$ & $0,7 \mathrm{~mm}$ \\
\hline Tôle de l'ourlet du bord du casque & $468-11 /$ TTC $12-2$ & $0,5 \mathrm{~mm}$ \\
\hline $\begin{array}{l}\text { Tôle du cou ; partie haute } \\
\text { de la section, proche du timbre }\end{array}$ & TTC 13 & $1 \mathrm{~mm}$ \\
\hline $\begin{array}{l}\text { Tôle du timbre ; au sommet, au } \\
\text { bord d'un des trous de la bélière }\end{array}$ & $468-8 /$ T4 & non mesurable \\
\hline $\begin{array}{l}\text { Couronne de l'œil droit } \\
\text { (prélèvement au scalpel) }\end{array}$ & $468-1 /$ T3 & \\
\hline $\begin{array}{l}\text { Matière blanche de l'œil gauche } \\
\text { (prélèvement au scalpel) }\end{array}$ & T2 & \\
\hline
\end{tabular}

Tableau 2. Caractéristiques des prélèvements réalisés sur le casque-oiseau. Les épaisseurs sont mesurées sur les coupes métallographiques observées en microscopie optique.

76- De même que pour le carnyx, les prélèvements ont été effectués lors de deux campagnes (cf. note 20) ; ceux de la première sont notés TTC, ceux de la seconde T.
Les sections métallographiques ont été préparées, puis étudiées, avec la méthodologie décrite précédemment dans le cas du carnyx.

La section transverse du prélèvement réalisé au bord du casque (fig. 62) montre que celui-ci est bordé par une pièce de tôle cintrée (fig. 63) ; il est à noter que cet "ourlet" est bien une pièce différente, et non le bord de l'ouverture qui serait roulé, comme on l'observe couramment dans le cas de certains vases. En plus d'un effet décoratif, cette pièce joue un rôle de raidisseur de l'ouverture afin de diminuer la possibilité de sa déformation ; naturellement, elle sert aussi à supprimer le caractère quelque peu tranchant de la tôle du bord du casque.

\section{Les compositions des alliages}

Toutes les pièces de tôle analysées, ainsi que la couronne de l'œil droit, correspondent à des alliages de cuivre et d'étain ; la teneur en masse de l'étain dans l'alliage est, dans tous les cas, située vers $10 \%$ avec une assez faible dispersion. Des traces de soufre et de plomb sont détectables, mais non dosables avec la technique employée. De même que dans le cas des pièces de tôle du carnyx, c'est un cuivre de bonne qualité qui a servi à élaborer les alliages de départ. La matière blanche, prélevée dans l'œil gauche (T2) est de l'étain non allié ; une large part du métal est, dans ce prélèvement, comme on pouvait s'y attendre, oxydée et transformée en produits de corrosion.

En dehors des yeux de la tête de l'oiseau, qui étaient polychromes, avec probablement un disque noir, entouré de blanc et cerné d'une couronne dorée, le reste de l'objet était, dans son état d'origine, certainement entièrement de couleur dorée, sans effet visible de nuances.

\section{Les procédés de formage}

Comme dans les tôles du carnyx, celles du casque présentent un état recristallisé ; le formage des pièces minces a donc été conduit par des passes de martelage alternées avec des traitements de recuit. Il est cependant à remarquer que la microstructure du prélèvement effectué à la base du timbre comporte des vestiges de la structure de fonderie. Ceci est certainement dû au fait que le bord de cette pièce, épais de $2 \mathrm{~mm}$, a subi beaucoup moins de déformation 


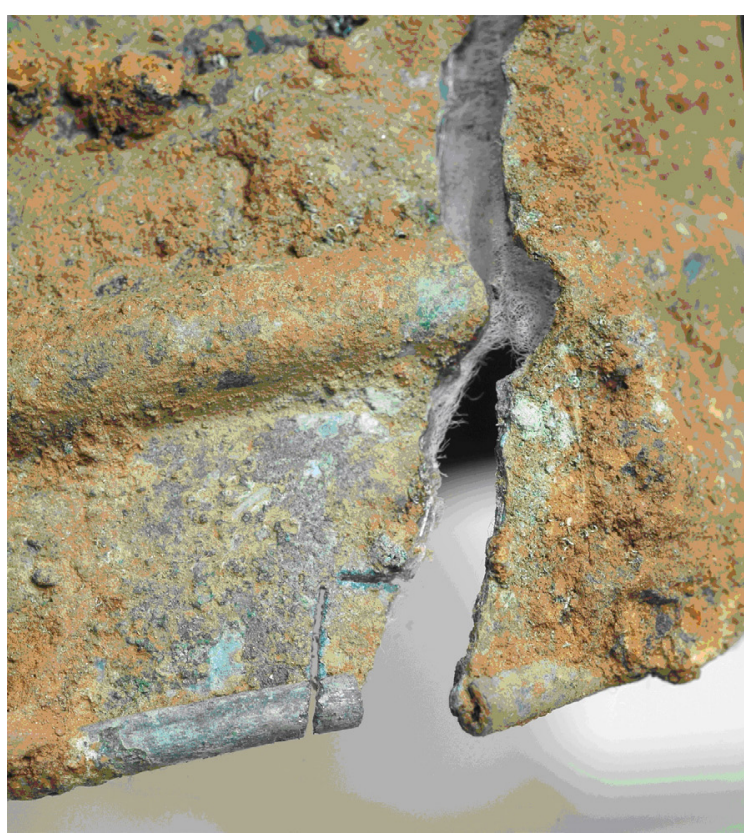

Fig. 62. Vue du prélèvement effectué sur le bord du casqueoiseau, avant qu'il soit détaché (cl. M. Pernot, CNRS).

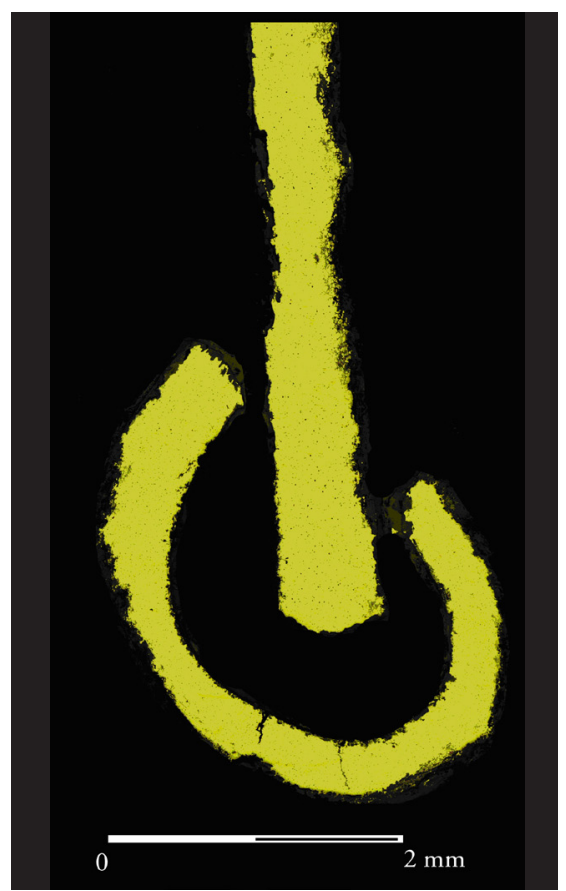

Fig. 63. Observation au microscope optique de la section polie perpendiculaire au bord du fragment prélevé visible sur la fig. 62. La pièce de tôle cintrée servant d'ourlet au bord du casque est clairement mise en évidence. que le sommet, voire pas du tout. En effet, comme c'est souvent le cas pour des pièces de vaisselle, si la déformation en expansion biaxiale a été conduite en "gonflant" la zone creuse, le bord de la pièce, pour assurer une meilleure rigidité, a, quant à lui, subi une réduction d'épaisseur très nettement moindre, voire n'en a pas subi ; ainsi, malgré les recuits, la microstructure de la périphérie peut alors n'être pas totalement transformée par les recristallisations successives. La présence de macles mécaniques dans les grains recristallisés indique qu'une faible déformation a été appliquée après le dernier recuit ; comme précédemment, le but principal de cette passe finale a été de "tendre les lignes" et de rendre l'objet plus rigide que si le métal restait dans un état adouci.

Bien que la microstructure ait été perturbée par le prélèvement réalisé au scalpel dans la couronne de l'œil droit, des nodules de seconde phase, riches en étain et avec la sous-structure caractéristique, ont été observés associés à des inclusions de sulfure de cuivre ne présentant pas d'allongement. Il est vraisemblable que cette pièce a été obtenue uniquement par une opération de fonderie, suivie peut-être par un travail de reprise en ciselure, pour accuser certains reliefs, avant le polissage final.

\section{Quelques remarques}

Il faut souligner que, dans le cas de ces deux objets, et pour ce qui en a été analysé, nous avons toujours observé l'usage de bronzes binaires ou de bronzes ternaires au plomb ; aucun emploi de laiton - alliage de cuivre et de zinc - n'a été mis en évidence. Rappelons que ce dernier type d'alliage a été utilisé pour la fabrication de la trompe du dépôt de Neuvy-en-Sullias dans le Loiret ${ }^{77}$, ainsi que pour celle de l'enseigne en forme de sanglier découverte à Soulac-sur-Mer en Gironde ${ }^{78}$.

Les procédés de fabrication employés, que ce soit la fonderie pour les pièces massives ou l'enchaînement de passes de martelage et de recuits pour les pièces en tôle mince, sont parfaitement classiques dans le cadre du $\mathrm{I}^{\mathrm{er}}$ millénaire a.C. Le choix d'alliages binaires de cuivre et d'étain, élaborés avec des cuivres de bonne "propreté", et avec une teneur en

77. Mille 2007.

78. Moreau et al. 1990. 
étain au voisinage de $10 \%$, est également la "norme" habituelle. De même, le choix de bronzes au plomb, avec environ $10 \%$ d'étain et $10 \%$ de plomb, pour les pièces réalisées en fonderie est courant, sans que ce soit systématique ; l'absence de plomb dans l'alliage de la couronne autour de l'œil, n'est pas une anomalie. Dans le cas du carnyx, un éventuel choix de cuivres différents pour élaborer les alliages des pièces produites par fonderie et ceux des pièces qui subissent un important travail de déformation plastique, ne serait pas étonnant ; mais cette hypothèse doit continuer d'être étudiée, d'être précisément cernée, afin de chercher si des contextes particuliers lui donnent un sens. Dans le même esprit, l'utilisation d'étain non allié, et non d'alliages d'étain et de plomb, ne peut être comparée à d'autres cas par manque d'études technologiques de ce type d'assemblage de cette période ; dans la suite de l'étude, l'attention devra être également orientée vers ce problème.

\section{Interprétations \\ (Th. Lejars)}

Ce couvre-chef métallique a l'apparence, et c'est bien là l'une de ses principales caractéristiques, d'un oiseau. Jusque là, nous avons accordé la préséance aux caractéristiques formelles sans nous interroger sur la nature ornithomorphe de l'objet. De quel oiseau s'agit-il ? Faut-il voir là un cygne comme il a semblé lors de la découverte? Rien n'est moins sûr. Nous l'avons vu, cette représentation est dénuée de tout réalisme. Il s'agit, sinon d'une évocation, tout au moins d'une interprétation. Le corps ovalaire de l'animal est trop schématique pour être significatif. Les ailes, pas plus que le plumage, ne sont figurées et on peut douter que les orifices latéraux situés à la base du timbre aient pu servir à la fixation de tels accessoires. L'appendice caudal, extrêmement massif, n'est pas davantage détaillé. Le cou, réduit à un simple tube, est à peine plus suggestif. Les surfaces sont parfaitement et uniformément lisses. Seule la tête a fait l'objet d'un traitement détaillé, même si le poli du métal ne laisse paraître aucune différence dans le traitement du bec et de la tête. Au contraire, on a vu avec l'arête inférieure du bec que les deux éléments s'imbriquent complètement. À cela s'ajoute le diamètre de la tête, à peine plus grand que celui du cou, qui confère à l'animal un aspect filiforme, quasi reptilien, avec des yeux plus grands que nature ${ }^{79}$.

En vue latérale, l'œil ainsi que le bec, long, pointu et légèrement arqué vers le bas, évoquent surtout les échassiers et plus particulièrement la grue ${ }^{80}$. En vue frontale, le bec arrondi et épaté rappelle quant à lui certains palmipèdes à bec long, comme le canard pilet. Du strict point de vue zoologique, il est donc difficile de parler de cygne dans le cas présent, comme il est tout aussi hasardeux d'identifier là quelque espèce que ce soit. Il semble donc, selon toute vraisemblance, que le concepteur se soit inspiré dans ce cas d'un échassier, peut-être une grue si l'on en juge par le bec assez court, alors que la tête plutôt fine pourrait indiquer un héron pourpré. Il faut également insister sur la conformation très équine de la base du bec qui n'est pas sans évoquer l'arrondi de la mandibule du cheval. C'est ce même souci anatomique qui signale les chevaux de la frise de Roquepertuse (fig. 64.1) ${ }^{81}$ ou encore le protomé en bronze du "Taunus", conservé à Berlin" ${ }^{82}$ : une tête chevaline pourvue de larges oreilles se dresse audessus d'un long col sinueux. L'espace délimité par les mandibules est marqué comme pour le bec de l'oiseau de Tintignac par une large dépression. L'objet daté de La Tène A n'a malheureusement pas de contexte connu qui puisse renseigner sa fonction initiale $^{83}$. L'art animalier des Celtes de l'époque de La Tène n'est certainement pas naturaliste et l'oiseau de Tintignac ne déroge pas à la règle. Il est clair que le concepteur n'a pas cherché à copier un modèle précis mais a pris les éléments significatifs qui répondaient le mieux à son dessein - il devait

79- Megaw 1981, 138, J.V.S. Megaw cite à propos du petit canard placé à l'extrémité du bec de la cruche de Basse-Yutz - qui semble nager sur le flot de vin versé - le commentaire du Dr Kear qui voit dans "le grand œil, le crâne proéminent, le bec mince dont les extrémités ne se rejoignent pas tout à fait, les particularités d'un caneton à demi-incubé, tandis que l'effet de taches produit par les marques au poinçon sur le corps, qui n'ont d'équivalent sur aucun canard adulte réel en Europe, pourrait avoir été destiné à représenter les papilles d'où les plumes doivent sortir"... et Megaw de s'interroger quant "au pouvoir générateur de vie de la boisson".

80- Il m’est agréable de remercier ici pour leurs précieuses remarques Patrice Méniel et François Poplin qui voient là en vérité un bien étrange oiseau, peu conforme à la réalité.

81- Duval 1977, 113.

82. Jacobsthal 1944, 199, n³72, pl. 175.

83. Megaw \& Megaw 1989, 82-83. 
connaître parfaitement les oiseaux, et a agi en connaissance de cause - et les a pliés à des règles de composition comme nous le verrons plus avant.

Notre difficulté à donner un nom à cet oiseau, ou à ce qui apparaît être un volatile hybride, n'enlève rien à l'intention de son créateur. Il s'agit bien d'un oiseau et plus précisément d'un spécimen lié à l'élément liquide, palmipède ou échassier ${ }^{84}$. Les représentations d'oiseaux aquatiques, en particulier le canard et le cygne, documentées depuis l'âge du Bronze sont encore fréquentes dans les figurations de la fin du Hallstatt et de La Tène ancienne (canard de la cruche de Basse-Yutz ${ }^{85}$, torques de la Marne ${ }^{86}$, fibule de Hundheim ${ }^{87}$ ). Ces animaux sont également figurés sous la forme de protomés à long col comme sur la bouterolle de Suippes ou encore l'applique de harnachement de Semides, en Champagne $\left.{ }^{88}\right)$. Il semble qu'ensuite l'oiseau d'eau, souvent mis en relation avec le char solaire ${ }^{89}$, perde de son importance au profit d'autres espèces comme les oiseaux de proie, figurés le plus souvent sous la forme schématique d'oiseau ou de protomés d'oiseaux à bec crochus. L'embout serpentiforme à tête chevaline du "Taunus" déjà signalé évoque une autre représentation, tout aussi difficile à dater, découverte au début du XIX ${ }^{\mathrm{e}}$ s. à Torrs, en Écosse, dans un ancien lac asséché (fig. 65.4) ${ }^{90}$. Ce chef-d'œuvre de la toreutique insulaire, daté du II $\mathrm{e}$ s. a.C. et interprété comme un chanfrein de poney, se compose de trois parties, la base ou timbre et deux longs appendices coudés. La reconstitution moderne d'un masque doté de cornes ne correspond certainement pas à son aspect d'origine et il n'est pas certain que les cornes ont été conçues pour être fixées au chan-

84- Megaw 1981, 137, note que "peu d'auteurs ont poussé très loin la question de l'identification réelle des espèces qui sont représentées dans l'art celtique." et poursuit en faisant remarquer qu'une "telle tâche peut sembler décourageante en raison du dédain apparent des artisans celtes pour le réalisme des représentations et de leur tendance naturelle à styliser les formes de la nature."

85- Jacobsthal 1944, n³81, pl. 181 ; Megaw \& Megaw 1989, 77.

86- Jacobsthal $1944,190, n^{\circ} 239$ et 240 , pl. 138, torques du MAN n ${ }^{\circ} 12985$ et 77024 .

87. Duval 1977, 52.

88- Lambot et al. 1995, fig. 58 et 66.

89. Duval 1982/1983, 66

90- Megaw \& Megaw 1989, 196-197. frein $^{91}$. À cela s'ajoute le fait que ces pièces richement ornées diffèrent par la technique décorative. Les cornes sont gravées tandis que le décor du chanfrein est réalisé au repoussé. Ce qui nous intéresse ici ce sont les cornes qui se signalent par un appendice terminal - un seul est conservé - en forme de protomé zoomorphe, peut-être un oiseau, avec deux grands yeux circulaires incrustés de matériaux colorés (verre ou corail). Comme la tête de l'oiseau de Tintignac, celle de Torrs a été fabriquée séparément et rapporté sur la tôle. Là encore, le traitement n'est pas naturaliste. Le bec, allongé, n'est pas droit, mais fortement arqué et prolonge harmonieusement la courbure du col. Le thème des oiseaux aquatiques trouve de nombreux répondants dans les recueils mythologiques. Il n'est pas dans mon propos de dresser ici un inventaire des occurrences qui envahissent les récits de l'Antiquité classique et celtique insulaire. Il suffit de rappeler que ces grands oiseaux migrateurs (cygnes, oies, cigognes, grues, etc.) sont souvent interchangeables et, pour certains d'entre eux, propices aux métamorphoses (cygnes et grues) ${ }^{92}$. Ils sont également considérés en Grèce, et plus tard, dans l'Irlande du Moyen Âge, comme des oiseaux mantiques en raison de leur chant particulier ou du fait même de leur simple apparition. On connaît les cygnes blancs qui tiraient le char de l'Apollon hyperboréen $^{93}$, on sait aussi qu'ils possédaient parfois une force destructive et que l'on voyait en eux des messagers de l'Autre-Monde. La grue, autre oiseau sacré d'Apollon, figure (elles sont trois) au sommet de l'arbre du monde sur plusieurs monuments galloromains. Elle est également réputée pour sa fidélité, sa vigilance et sa combativité. L'affrontement qui oppose les grues, toujours victorieuses, aux Pygmées est un des thèmes récurrents de l'iconographie hellénistique. C'est aussi un des thèmes de prédilection des peintres étrusques (fig. 64.8-9)94. La force de ces oiseaux, perçus comme l'image symbolique d'un groupe humain en migration, a aussi très certainement frappé l'imaginaire des Celtes, eux-mêmes ar-

91- Hunter 2009c, 230. F. Hunter propose de dater l'objet autour des années 200 a.C. et souligne la parenté stylistique des motifs gravés avec le Style des épées, tandis que les décors au repoussé du chanfrein sont mis en relation avec le Style plastique. 92. Duval 1982/1983, 68-69; Mathieu 1990 ; Boekhoorn 2008. 93. Alcée, Poèmes, Livre I, texte et traduction par Th. Reinach, Les Belles Lettres, Paris, 28-29.

94- Steingräber 1985, 338, pl. 136. 


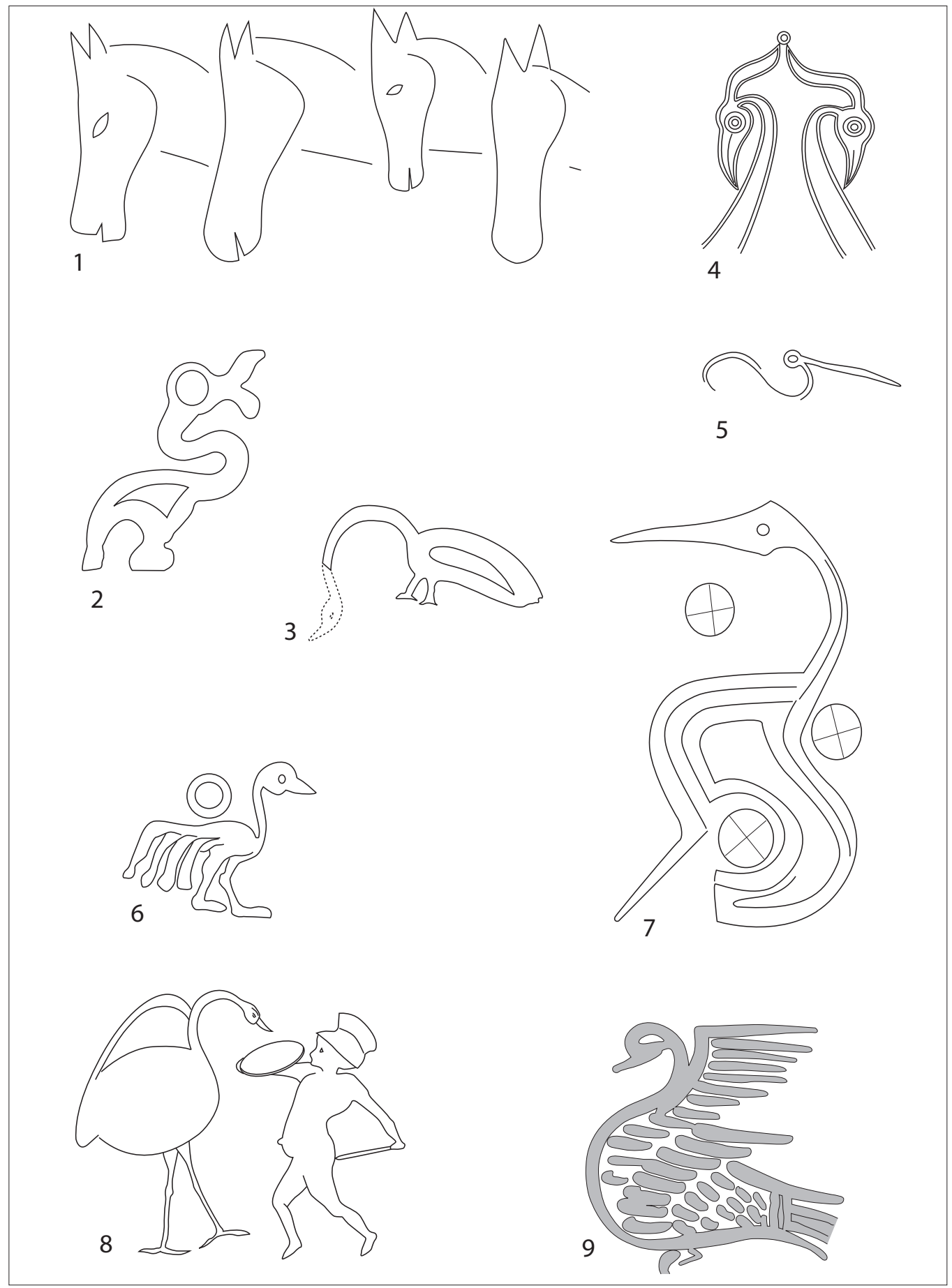

Fig. 64 1. Frise de chevaux, Roquepertuse (d'après Duval 1977) ; 2 . Motif ornithomorphe de l'anneau passe-guide de Waldalgesheim, (Allemagne, d'après Jacobsthal 1944) ; 3 . Motif ornithomorphe d'une coupe de Radovesice (République Tchèque, d'après Duval 1982/83) ; 4. La Tène (Suisse, d'après Bulard 1982) ; 5. Motif ornithomorphe d'une coupe à pied du Musée des Antiquités Nationales (d'après Duval 1982/1983) ; 6. Motif ornithomorphe d'une monnaie lémovice ; 7. Paragnathide de casque, Smarjeta (Slovénie, d'après Duval 1977) ; 8 . Combat des grues et des Pygmées (détail), tombe des Pygmées, Tarquinia (Steingräber 1985) ; 9. Motif ornithomorphe peint sur une coupe étrusque à vernis noir (nécropole de Monterenzio Vecchia, Italie). 


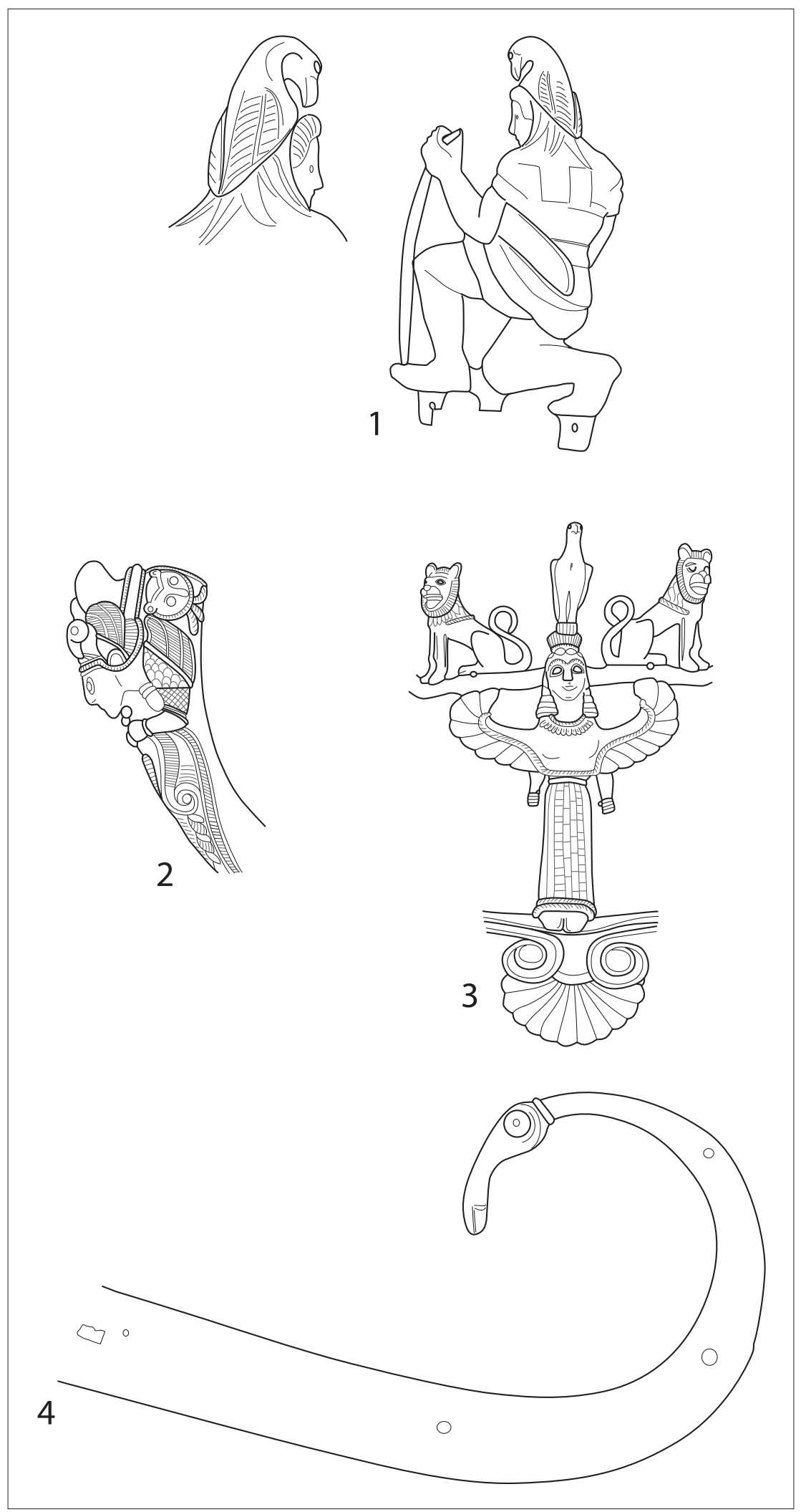

Fig. 65 1. Altino, statuette d'archer agenouillé (Italie, d'après Tirelli 2005) ; 2. Reinheim, extrémité de bracelet (Allemagne, d'après Verger 1991) ; 3. Grächwil, anse d'hydrie grecque (Suisse, d'après Verger 1991) ; 4. Torrs, appendice cornu à protomé zoomorphe (Grande-Bretagne, d'après Duval 1977). 
dents combattants et impétueux migrants. C'est peut-être à ce symbolisme que renvoient certains animaux fantastiques au long cou de la fin du $\mathrm{V}^{\mathrm{e}}$ s. a.C., comme ceux que l'on rencontre sur un fer de lance de Saint-Sulpice ${ }^{95}$ et un des fourreaux d'épée de La Tène (fig. 64.4) ${ }^{96}$, ou encore certaines représentations plus tardives comme les grues en pied, frappant des ailes, qui ornent les paragnathides de quelques casques de Slovénie du dernier siècle a.C. (fig. 64.7) ${ }^{97}$. C'est une grue, ou plus probablement un ciconiiforme, que propose de voir Alain Duval dans la série d'oiseaux figurés sur le sommet de la panse d'un vase caréné avec piédestal conservé au Musée de Saint-Germain-en-Laye (fig. 64.5). Réduits à quelques traits essentiels, l'oiseau est constitué d'un corps en forme d'esse couchée et d'un disque pointé, évoquant la tête et l'œil du volatile, sur lequel vient se greffer un long bec droit, effilé. Sans contexte et sans provenance connue, le vase a été daté du $\mathrm{II}^{\mathrm{e}} \mathrm{s}$. a.C. Une datation légèrement antérieure, dans le courant de la seconde moitié du $\mathrm{III}^{\mathrm{e}} \mathrm{s}$. a.C., est cependant plus probable si l'on juge pertinentes les comparaisons avec les vases des tombes de guerrier de Marson-Montfercault et Somsois ${ }^{98}$.

C'est à cette catégorie d'oiseaux qu'appartient aussi très certainement l'enseigne de Castiglione delle Stiviere, près de Mantoue ${ }^{99}$. Ce qui reste du mobilier témoigne du prestige du destinataire de cette tombe du milieu du $\mathrm{III}^{\mathrm{e}} \mathrm{s}$. a.C. Les restes incomplets de l'oiseau de Castiglione ont été d'abord interprétés comme un carnyx par Raffaele De Marinis. Cette lecture a été réfutée par Daniele Vitali et Fraser Hunter ${ }^{100}$. Alors que le premier propose de distinguer dans les

95- Saint-Sulpice, tombe 57 (Suisse), Kaenel 1990, pl. 49.

96- La Tène $n^{\circ} 4$, De Navarro 1972, pl. II.2 et LXXII et LXXIII.

97- Pernet 2010, pl. 91, Bela Cerkev, Kosak B tombe 1 (également connu sous le nom de Smajeta); pl. 118, Mihovo tombe 1656/58.

Vue détaillée de la paragnathide de Bela Cerkev dans Duval 1977, 157.

98. Duval 1982/1983. Les tombes de Somsois et MarsonMontfercault se caractérisent l'une et l'autre par un umbo de bouclier à ailettes légèrement trapézoïdales caractéristiques de la fin de La Tène C1. La première se signale en outre par la présence d'une chaîne de suspension de type "gourmette", une forme évoluée qui n’apparaît plus dans le second ensemble. Les fibules de schéma La Tène II de la tombe de Somsois permettent également d'assigner l'ensemble au $\mathrm{III}^{\mathrm{e}}$ s. a.C. MarsonMontfercault, cf. Charpy \& Rapin 1991. Le mobilier des deux tombes est conservé à Londres, British Museum, voir Stead \& Rigby 1999, 153, 162-164.

99. De Marinis 1997, 164

100- Vitali 2002, 21-22, pl. I ; Hunter 2009a, 81-82, fig. 10. débris conservés les restes d'un carnyx (le pavillon circulaire, la pièce angulaire à double orifice et une partie tubulaire) et d'une enseigne (la base du col, une aile mobile et les pattes figurées sur un disque support), le second ne voit là qu'une enseigne (la pièce coudée constituant la tête stylisée de l'oiseau, le tube, une des pattes, et le pavillon, la base sur laquelle se trouvait perché l'oiseau) ${ }^{101}$. Toutefois, F. Hunter n'exclut pas une utilisation comme ornement de casque, bien que rien dans le mobilier conservé ne vienne confirmer l'existence d'un tel objet. L'oiseau avec ses ailes mobiles fait référence, selon D. Vitali, soit à une enseigne militaire, soit à un élément décoratif de casque, comme celui de Ciumesti daté du début du III ${ }^{\mathrm{e}}$ s. a.C. (fig. 66). Le thème de la grue trouve par ailleurs un prolongement inattendu dans l'iconographie monétaire lémovice, avec le type dit à la "grue". L'oiseau, parfaitement identifiable en dépit de la taille de l'image, se dresse, les ailes déployées vers l'arrière, au-dessus d'un cheval (fig. 64.6). Si l'attribut monétaire renvoie assez logiquement à la vertu belliqueuse de l'oiseau, cette lecture ne paraît pas en revanche s'imposer pour le casque-oiseau de Tintignac qui relève davantage de la sphère symbolique que de celle de la guerre stricto sensu. La position de l'animal, avec la tête tournée vers l'arrière, oriente vers d'autres interprétations, comme nous nous proposons de le montrer par un examen des codes de représentation.

L'appendice cornu de Torrs invite à penser que la tête de l'oiseau avec son bec incurvé n'a pas été conçue au hasard. Il convient donc de s'arrêter sur les règles de composition et les points qui font l'originalité du casque de Tintignac et lui confèrent aussi tout son sens. Le premier concerne la position de la tête, nullement naturelle. L'orientation de cette dernière, avec la gorge dirigée vers le bas, implique que

le cou n'est pas simplement cambré en arrière. Il faut donc supposer que le long col de l'oiseau n'a

101- P. Jacobsthal parle quant à lui de bird-askoi et retient pour improbables les suggestions de l'inventeur qui propose de voir les ailes d'une enseigne en forme d'oiseau (Jacobsthal 1944, 145, n³98, pl. 201, 256-257). 


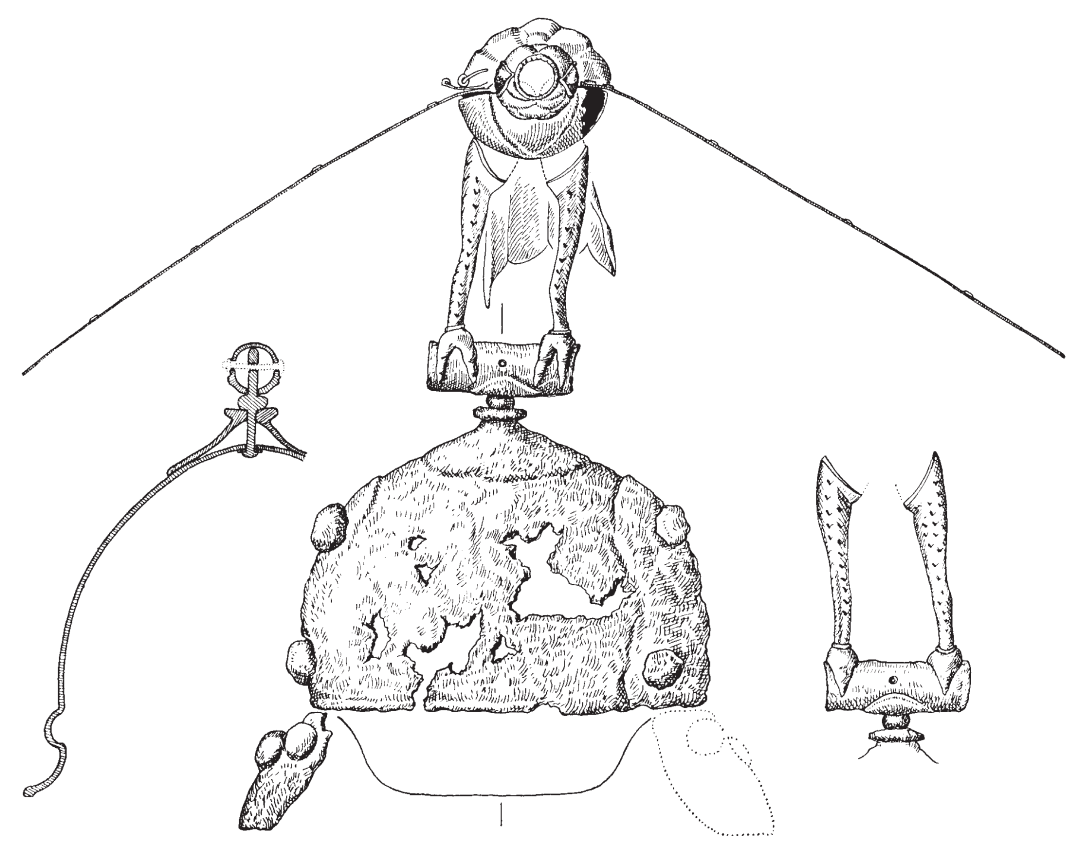

Fig. 66. Casque de Ciumesti

(Roumanie, d'après Rusu 1969).
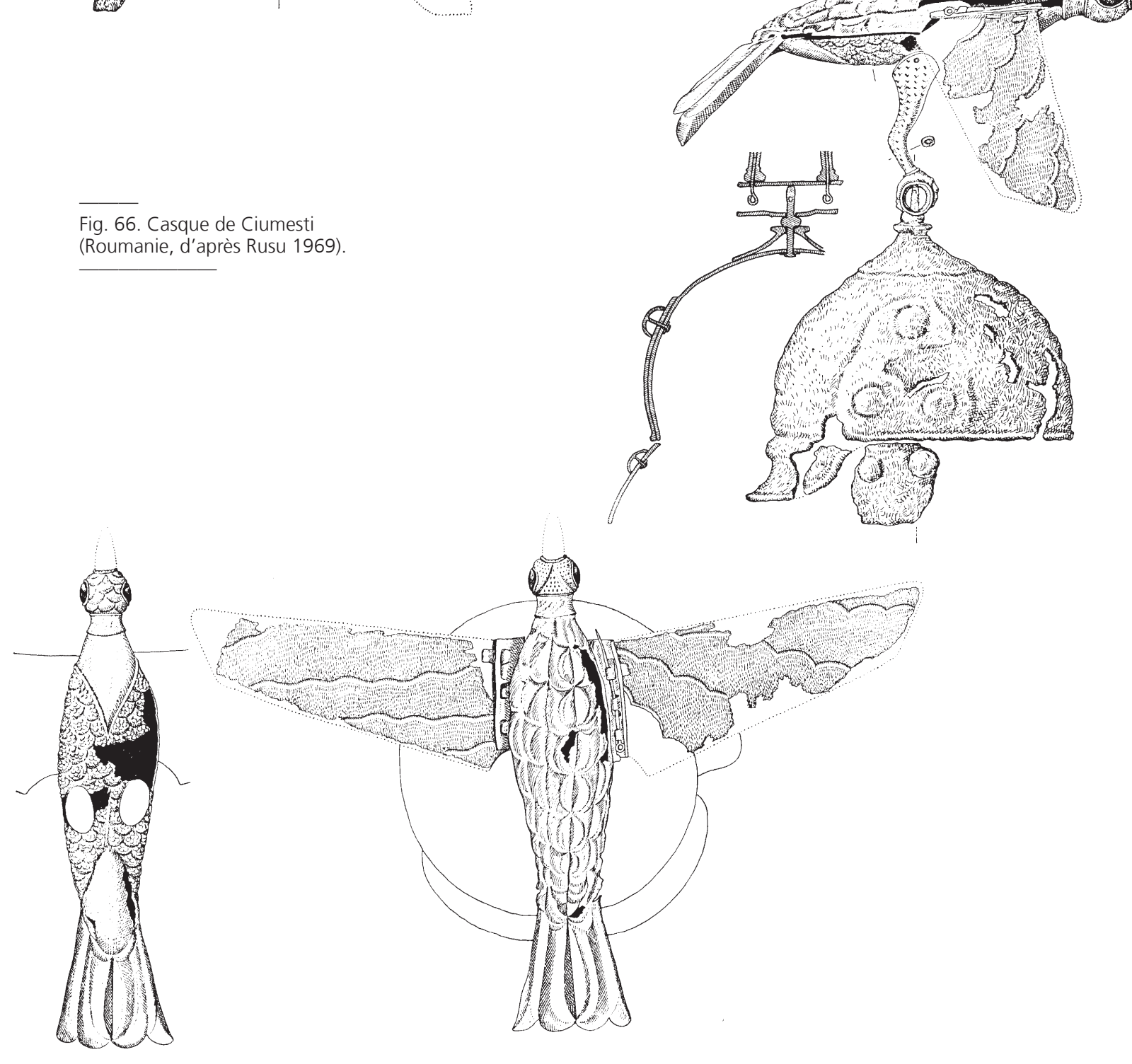
pas été renversé mais qu'il a subi un déplacement latéral de $180^{\circ} 102$, de manière à porter la tête vers l'arrière et la placer dans l'axe longitudinal. Pour parvenir à cette position contrainte, l'artisan ne s'embarrasse guère plus des règles de l'anatomie que pour les autres parties constitutives de l'animal. L'oiseau est ainsi artificiellement figuré à plat, la profondeur de champ induite par le mouvement giratoire du col étant tout simplement éliminée. Malgré l'artifice, cette mise en scène ne choque pas l'observateur, qu'il prenne place à l'arrière ou sur les côtés. De face, en revanche, l'oiseau disparaît et fait place à une sorte de rostre ou de corne frontale (fig. 67 et 68). La courbure du col n'est pas davantage habi-

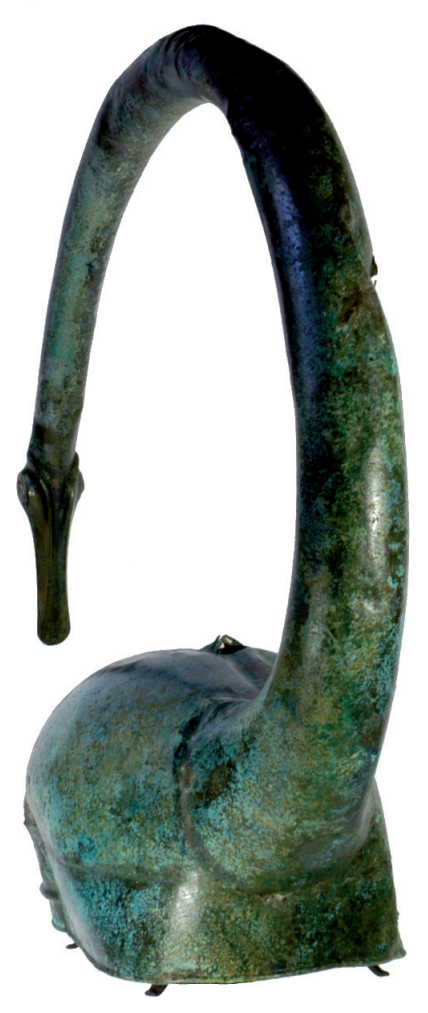

Fig. 67. Le casque vu de face (cl. B. Armbruster, CNRS).

Fig. 68. Le casque vu de l'arrière (cl. B. Armbruster, CNRS).

102- Les coups et le poids du comblement ont légèrement modifié l'axe du col de l'oiseau. tuelle, ni ne correspond à la formule classique du col de cygne en S (fig. 64.2) ${ }^{103}$. Cette présentation, guère réaliste, est toutefois moins surprenante qu'il n'y paraît et s'explique fort bien si on la considère du point de vue des règles de composition de l'art celtique ancien. La courbure tendue et régulière du col dessine, avec la tête qui la prolonge, une large boucle au-dessus du corps de l'oiseau. Le bec pointe en direction de l'extrémité de la queue sans toutefois parvenir à la joindre. L'espace interne, délimité par le tracé du col et son prolongement en direction du timbre et de l'appendice caudal, reproduit en négatif le motif de la feuille ou virgule, ou encore "vessie de poisson”, un motif bien connu de l'art celtique de-

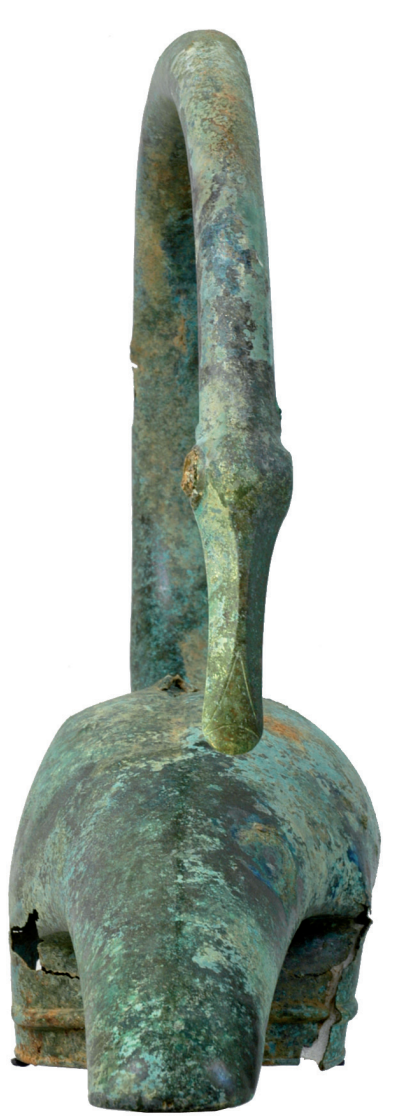

103- Par exemple les lyres zoomorphes des fourreaux d'épées ou encore les oiseaux affrontés de l'anneau passe-guide de Waldalgesheim (fig. 3.2) ; Megaw \& Megaw 1989, 114. Il y a assurément un problème avec les articulations des vertèbres cervicales qui, même si elles donnent une grande souplesse au cou, ne permettent pas une telle position, comme nous l'indique Patrice Méniel. 
puis les époques les plus anciennes (en fait une esse fermée par un segment de cercle). Le motif est utilisé seul ou combiné à d'autres signes. Utilisé par paire, et disposés symétriquement, il forme la couronne de feuilles ${ }^{104}$ qui encadre et ceint généralement une tête représentée de face. Or, si dans le cas présent, nous avons affaire à une feuille unique, il n'est pas inopportun de souligner qu'elle est associée à une parure de tête et coiffait un personnage, réel ou fictif (fig. 69.1). Pour s'en convaincre, on se souviendra qu'un semblable couvre-chef ceignait la tête du personnage de la tombe 1 de Glauberg, tout comme la statue qui en signalait le tumulus (fig. 69.2) ${ }^{105}$. Cet attribut fréquent à La Tène ancienne ${ }^{106}$ (fig. 69.3-6) est encore attesté à La Tène $\mathrm{C}$, mais généralement de manière simplifiée comme on peut le voir sur l'ornement gravé sous l'entrée d'un des fourreaux hongrois de Bölcske Madocsahegy (fig. 69.7) ${ }^{107}$. Ce choix de composition ne doit donc probablement rien au hasard et il ne faut pas non plus s'étonner de voir réduit au minimum les détails désignant l'oiseau, comme les ailes qui ne sont pas même esquissées.

La posture de l'oiseau avec le cou tourné vers l'arrière n'est pas sans rappeler un petit groupe de représentations de l'art animalier de La Tène ancienne. Il en va ainsi d'un certain nombre de figures de béliers (le bracelet de Rodenbach ${ }^{108}$ ), d'oiseaux (le torque de Besseringen ${ }^{109}$ ) ou d'animaux fabuleux (les sphinx de l'agrafe de ceinture de Weiskirchen ${ }^{110}$ (fig. 70.2), de la fibule et des cruches de la tombe 1 de Glauberg $\left.{ }^{111}\right)$. Les exemples cités proviennent pour la plupart du répertoire le plus ancien de l'art celtique des régions rhénanes où les figures animales, plus ou moins schématisées, jouissaient d'un grand succès. Ce type de représentation est aussi documenté en Champagne comme on peut le voir avec la théorie de quadrupèdes qui orne un vase de la collection

104- Sur le modèle des feuilles de gui, la plante sacrée des Celtes selon Pline, Nat., 16.95.

105- Frey 2008a, 64-71 ; Müller 2009, 190-192.

106- Exemples nombreux dans Jacobsthal 1944, pl. 9-14, 21, 30, 67, 98, 103, 121, 190 ; Müller 2009, 272-273

107- Szabo \& Petres 1992, cat. n5, pl. 7 ; Szabo 1993, 271-286.

108- Megaw \& Megaw 1989, 89.

109. Megaw \& Megaw 1989,90

110- Duval 1977, 46 ; Megaw \& Megaw 1989, 66

111- Das Rätsel der Kelten 2002, 148, 245, 250, 257 ; Megaw \& Megaw 1989, 259.
Nicaise, provenant de L'Épine (fig. 70.1) ${ }^{112}$. Le thème du cheval avec la tête tournée vers l'arrière est encore attesté à La Tène moyenne (un des trois chevaux placés à l'entrée d'un des plus fameux fourreaux du site de La Tène ${ }^{113}$; fig. 70.6) et plus tard encore, sur certaines monnaies ${ }^{114}$ et les ornements de seau d'Aylesford en Grande-Bretagne (fig. 70.7) ${ }^{115}$. Dans tous les cas, la tête subit un mouvement giratoire forcé, de sorte que l'animal dessine une large esse. La nature sinueuse apparaît sans aucune ambiguité sur les formes animales géométrisées de certaines agrafes de ceinture (fig. 70.3) ou encore la plaque ajourée de Cuperly ${ }^{116}$. Enfin, il convient de mentionner, pour clore ce bref excursus, une pièce ouvragée en bronze provenant aussi de Champagne ${ }^{117}$, figurant une paire d'oiseaux opposés qui viennent se lover dans le creux d'un orle incurvé, orné aux extrémités de quatre disques rehaussés de corail (fig. 70.4). Les oiseaux se caractérisent en outre par un corps ajouré en forme de feuille ou de virgule, surmonté d'une tête à bec crochu dirigée vers l'arrière. Le résultat est identique à celui obtenu à Tintignac. Ces animaux réels ou fabuleux se distinguent toutefois de l'oiseau de Tintignac par leur cou relativement court. Dans la production animalière laténienne, les oiseaux au long cou paraissent plus rares. Il faut mentionner ici les trois cygnes du vase de Radovesice (fig. 64.3) ${ }^{118}$, au tracé sommaire, et la paire d'oiseaux affrontés de l'anneau passe-guide de Waldalgesheim (fig. 64.2) ${ }^{119}$. Dans les deux cas, la posture des oiseaux est tout à fait normale du point de vue anatomique puisque les premiers semblent picorer paisiblement tandis que les seconds se dressent sur leurs

112- Jacobsthal 1944, 205, n410, pl. 208 ; Duval 1977, 75 ; Lambot et al. 1995, 56, fig. 60.2. Il s'agit dans ce cas d'une sorte de cheval à tête de griffon (bec crochu ouvert et oreille ou corne sinueuse).

113. De Navarro 1972, en frontispice ; Megaw \& Megaw 1989, 133.

114- Par exemple Duval 1977, 142, statère attribué aux Turones. 115. Duval 1977, 207. Figuration en léger relief de chevaux affrontés avec les têtes tournées vers l'arrière.

116- Par exemple l'agrafe de ceinture des environs d'Epernay ; Lambot et al. 1995, 57, fig. 62.4. Plaque de Cuperly, ibid., fig. 59. 117- Jacobsthal 1944, n¹02, pl. 63 ; Lambot et al. 1995, 58, fig. 63.

118- Ibid., 95

119- Ibid., 114. 

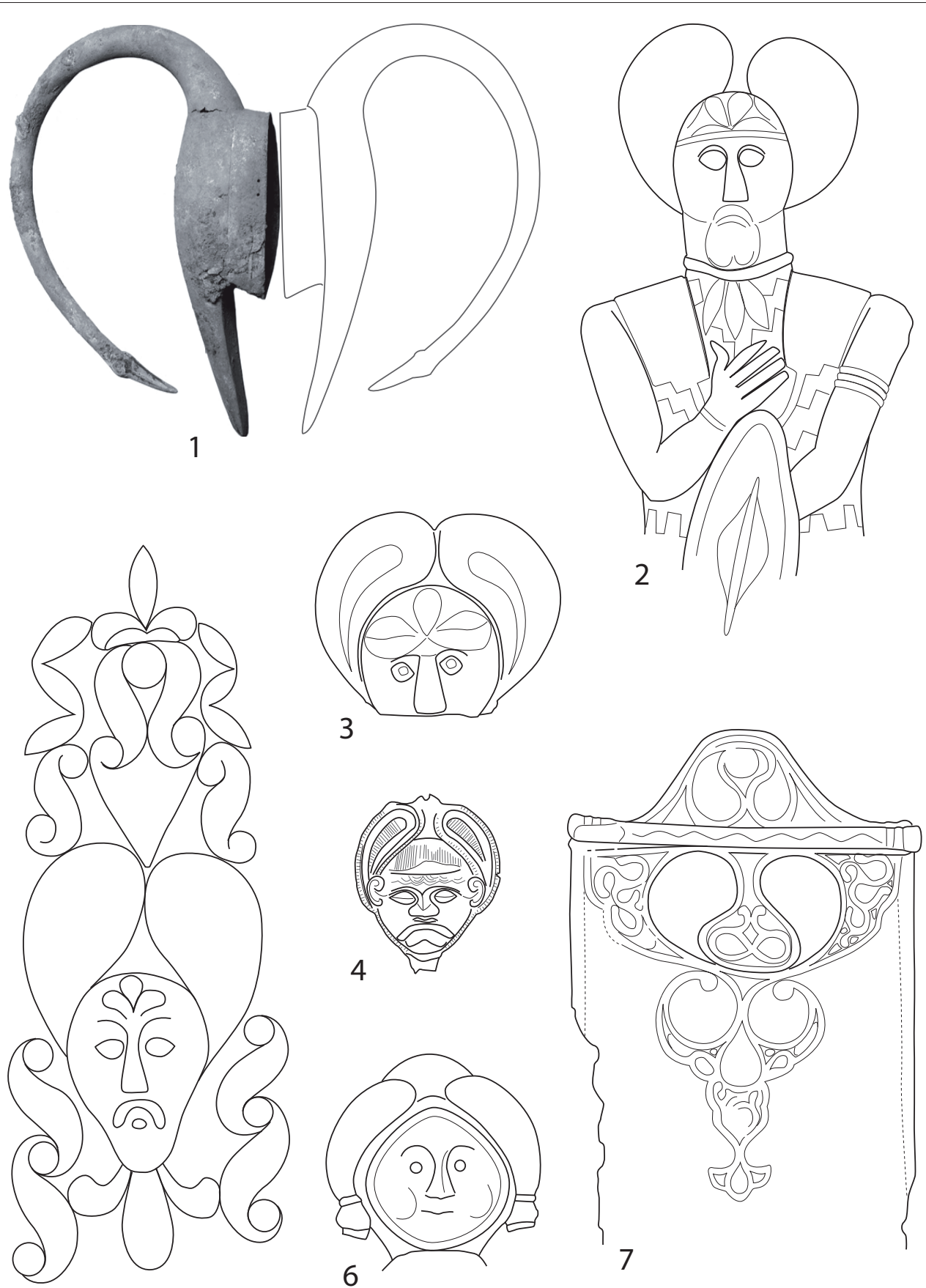

5

Fig. 69. Les têtes couronnées. 1. Restitution de la couronne de feuilles à partir du tracé du casque de Tintignac ; 2. Statue de Glauberg (Allemagne, d'après Das Rätsel der Kelten 2002) ; 3. Heidelberg (Allemagne, d'après Jacobsthal 1944) ; 4. Schwarzenbach (Allemagne, d'après Jacobsthal 1944) ; 5. Pfalzfeld (Allemagne, d'après Jacobsthal 1944); 6. Bescheid (tum. 6, Allemagne, d'après Das Rätsel der Kelten 2002) ; 7. Bölcske Madocsahegy (Hongrie, d'après Szabo \& Petres 1992). 


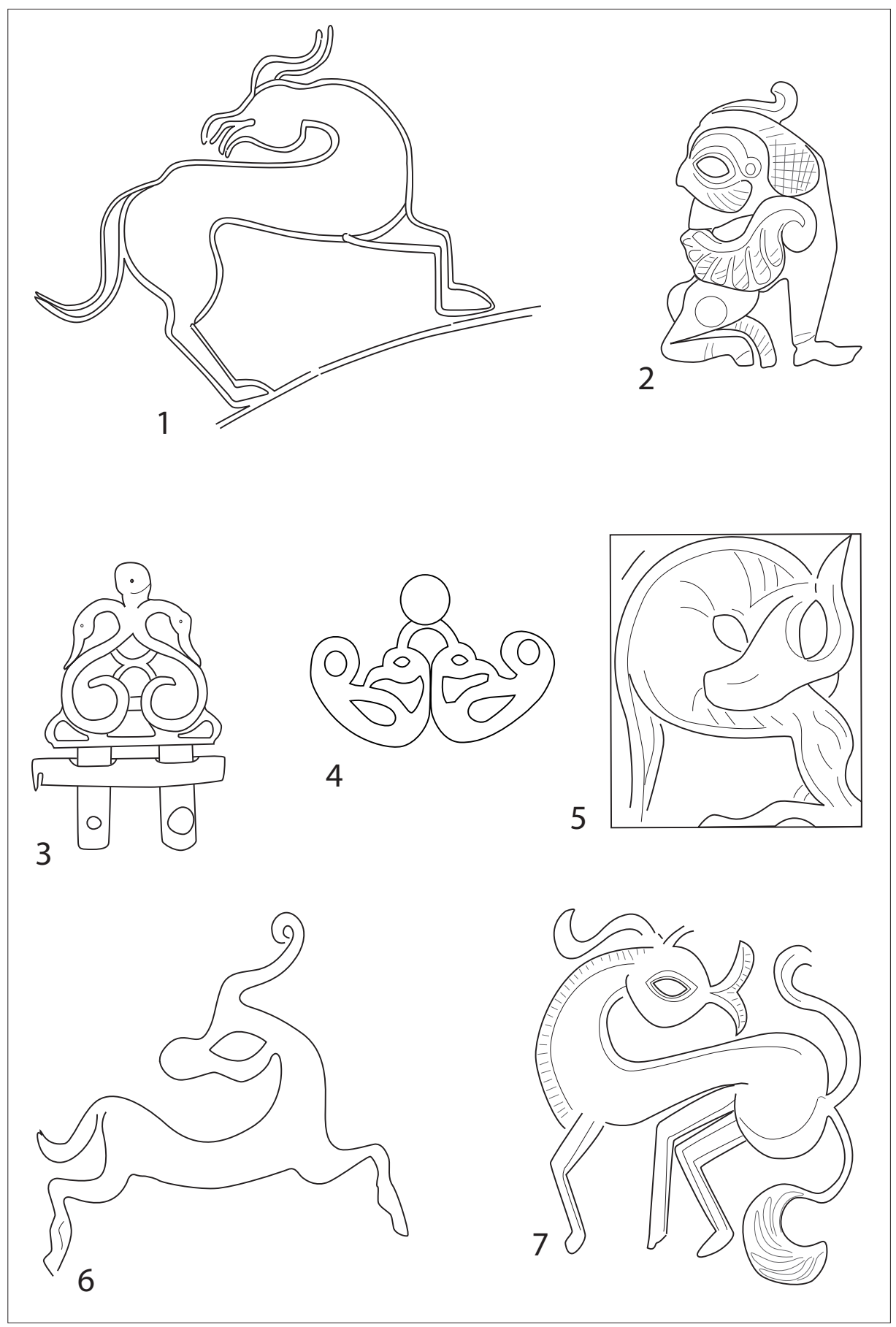

Fig. 70. Motifs animaliers avec la tête tournée vers I'arrière. 1. L'Épine, détail d'un vase à décor incisé (d'après Duval 1977) ; 2 . Weiskirchen, détail de l'agrafe de ceinture (Allemagne, d'après Duval 1977) ; 3. Région d'Épernay, agrafe de ceinture (d'après Lambot et al.1995) ; 4. Musée des Antiquités Nationales, applique (d'après Jacobsthal 1944) ; 5 . Herzogenburg-Ossarn, détail de l'agrafe de ceinture (Autriche, d'après Frey 2008) ; 6. La Tène (Suisse, d'après Duval 1977) ; 7. Aylesford (Grande-Bretagne, d'après Duval 1977). 
pattes, le cou sinueux tendu vers le haut ${ }^{120}$. Ce n'est en revanche pas le cas d'un des motifs animaliers de la plaque de ceinture de Herzogenburg-Ossarn, en Autriche ${ }^{121}$. L'animal est placé sur le côté, perpendiculairement à la paire d'animaux affrontés qui occupent le centre du registre. Il ne s'agit plus d'un oiseau mais d'un animal - un mammifère non défini ou une sorte de griffon au bec crochu fermé ? - qui se signale par un membre postérieur très développé (fig. 70.5). Le cou allongé s'étire vers l'arrière tandis que la tête hypertrophiée apparaît au-dessous. Elle n'est pas tournée vers l'arrière comme on pourrait s'y attendre mais vers l'avant en raison du cadre contraignant qui limite le champ de l'image. Ces exemples issus pour la plupart du répertoire laténien ancien montrent que la formulation du casque-oiseau de Tintignac ne constitue pas une anomalie mais, au contraire, s'inscrit dans une longue tradition remontant au début du second âge du Fer.

Tout en opérant dans un cadre conceptuel parfaitement balisé, avec son langage plastique et ses codifications formelles, le réalisateur du casque-oiseau de Tintignac n'en crée pas moins une ouvre originale, unique en son genre. En focalisant notre attention sur cette pièce exceptionnelle, il ne faut cependant pas oublier qu'elle provient d'un ensemble tout aussi spectaculaire et que la petite dizaine de casques associés est toute aussi insolite, par le nombre d'exemplaires et leurs formes tout à fait inhabituelles. Cela l'est d'autant plus que l'archéologie celtique est plutôt avare de ce genre de découverte et que pour cette catégorie d'objets les formes animales sont plus rares encore. Il a fallu attendre la découverte à Ciumesti ${ }^{122}$, en Roumanie, aux confins orientaux du monde celtique, d'un casque en fer surmonté d'un oiseau en bronze aux ailes déployées et articulées, pour que l'on accorde quelque crédit aux dires de Diodore de Sicile et aux représentations qui figurent sur le célèbre chaudron de Gundestrup

120- Les oiseaux du passe-guide de Waldalgesheim sont coiffés chacun d'une esse qui forme avec l'arrondi de l'anneau un motif en gousse. L'association des deux motifs au-dessus des oiseaux n'est pas sans évoquer la couronne de feuille de gui précédemment décrite. Jacobsthal 1944, 97.

121- Frey 2008b, 56.

122. Rusu 1969, 267-300; Megaw \& Megaw 1989, 177.
(Danemark) ${ }^{123}$ et diverses monnaies ${ }^{124}$ (fig. 71.1-3). Diodore nous dit des Gaulois qu'ils "se coiffent de casques d'airain avec de grands ornements en hauteur, lesquels donnent à ceux qui s'en servent une apparence gigantesque ${ }^{125}$. À quelques-uns même de ces casques sont fixées des cornes de même nature, et à d'autres des masques en relief d'oiseaux ou de

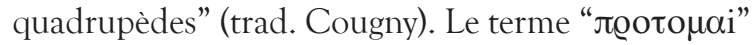
utilisé par Diodore et traduit par "masques" désigne plus précisément la partie antérieure de l'animal. Ces casques spectaculaires mais également peu communs, qui évoquent certains spécimens grecs ou gréco-italiques réels ou simplement figurés ${ }^{126}$, auront marqué à l'évidence ce grec ou plus probablement sa source dans la mesure où sa description des armes renvoie à une époque nettement plus ancienne, remontant probablement au $\mathrm{III}^{\mathrm{e}} \mathrm{s}$. a.C.

Les casques oiseaux connus dans les mondes celtique et classique sont, il faut bien l'admettre, fort différents de l'exemplaire de Tintignac dans la mesure où il est exceptionnel que le casque et l'ornement se confondent de cette manière (fig. 71.4) ${ }^{127}$. Les deux éléments sont plus souvent juxtaposés. Le rapace aux ailes déployées et articulées de Ciumesti est comme perché au-dessus du casque (fig. 66). Dans ce cas, le plumage est clairement indiqué. Le galbe des pattes et la position des serres sur le support confèrent un certain réalisme à l'oiseau mécanique. Le casque appartient à la série des casques à

123- Duval 1977, 185-187 ; Megaw \& Megaw 1989, 176. 124- Megaw \& Megaw 1989, 161, monnaie danubienne à la main. Le casque est surmonté d'un sanglier.

125- Diod., Bibliothèque historique, 5.30. On proposera de

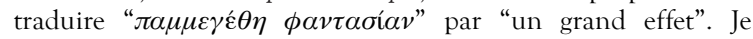
remercie Manuela Diliberto de son aide pour la lecture et la compréhension du texte de Diodore. Dans le monde grec, les hauts cimiers figurés sur les vases peints et certaines sculptures avaient pour but de grandir les guerriers, de terrifier leurs adversaires et de servir de signe de ralliement pendant le combat. Pour en augmenter l'effet, on teignait le panache et les plumes de couleurs vives, tantôt en rouge, tantôt en rouge et en noir. Cf. Reinach, art. "Galea”, Dict. ant. grecques et romaines.

126- Pour un aperçu de ces casques parfois très spectaculaires, qui ne manquent pas d'étonner, cf. Born \& Hansen 1994.

127- On peut mentionner certaines monnaies en bronze bellovaques, dites "au coq et à tête humaine", qui présentent au revers un personnage coiffé d'un oiseau battant des ailes ; Delestrée 1980. L'oiseau traditionnellement identifié comme un coq évoque plus probablement un cheval ailé ; la tête de l'animal avec ses appendices en volutes n'est pas non plus sans analogies avec les représentations de griffons et autres animaux fantastiques du répertoire ancien. 


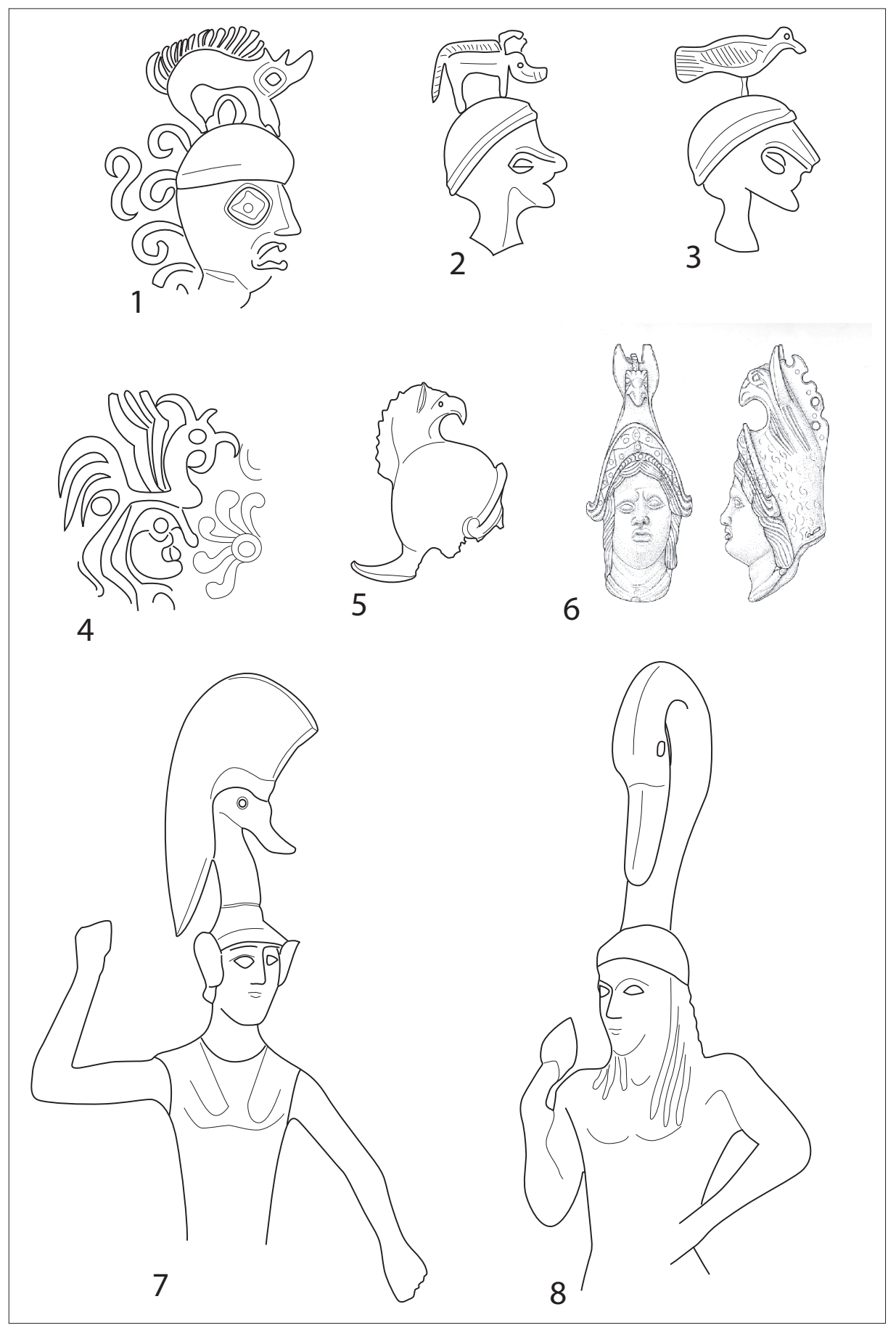

Fig. 71. Casques à ornements zoomorphes. 1. Guerrier avec casque surmonté d'un sanglier, monnaie d'argent (Hongrie, d'après Megaw 1989) ; 2 et 3. Guerriers équipés de casques à décors animaliers, chaudron de Gundestrup (Danemark, d'après Duval 1977) ; 4. Monnaie gauloise bellovaque, type dit "au coq à tête humaine" (d'après Delestrée 1980) ; 5 . Casque grec de type phrygien à protomé de griffon (d'après Feugère 1994) ; 6. Stari, poids en bronze (Slovénie, d'après Gaspari 2009) ; 7 et 8. Bronzes étrusques (Italie, d'après De Marinis \& Spadea 2004). 
bouton et couvre-nuque rapporté, en fer, usuels dans les régions balkaniques et connu par une représentation figurée sur un des bas-reliefs de la balustrade du portique du temple d'Athéna à Pergame ${ }^{128}$. L'oiseau du casque de la statuette en bronze d'époque romaine de Kerguilly ${ }^{129}$ (Finistère) - un canard ou une oie sauvage - prend appui sur le casque et sert de base au cimier. Dans d'autres cas, l'oiseau peut se réduire à un simple protomé, placé au sommet du casque et dirigé vers l'avant. La petite statuaire tardoarchaïque étrusque est connue pour certaines représentations de guerriers équipés d'un casque surmonté d'un haut col de cygne sur lequel vient parfois prendre appui un imposant cimier (fig. 71.7-8) ${ }^{130}$. Ce type d'ornement qui fait saillie au-dessus du personnage rappelle également la description que fait Diodore des casques gaulois. On a parfois évoqué pour ce motif le roi légendaire des Ligures, Cycnos, que les dieux métamorphosèrent en cygne ${ }^{131}$. Il existe aussi des casques coiffés d'une tête de rapace (fig. 71.5) ${ }^{132}$. Parmi les casques à protomé animale, nous trouvons une série d'objets figurant une tête casquée ${ }^{133}$. Il s'agit de poids en bronze et plomb. Le couvre-chef - il n'est pas certain qu'il s'agisse effectivement d'un casque - est surmonté d'une tête de griffon (fig. 71.6). L'animal fantastique est clairement identifiable avec son bec crochu, ses oreilles taillées en pointes et sa crête dorsale. On a proposé de voir dans ce type de tête masculine, daté entre la seconde moitié du $\mathrm{I}^{\mathrm{er}} \mathrm{s}$. a.C. et le début de l'époque impériale, une évocation du héros Persée qui se ma-

128- Bohn 1885 ; Kähler 1948.

129- Divinité de Dinéault, Kerguily dans Duval 1977, 194 ; Au temps des Celtes 1986, 133, 143. L'oiseau - un canard ou une oie sauvage, le cou est trop court pour être un cygne - sert de base à un porte-cimier.

130- Exemples dans, Born \& Hansen 1994, fig. $43-44$ et 59-60 ; De Marinis \& Spadea 2004, 28-29 et Lubtchansky 2005, 119.

122 , qui signale, outre une statuette de guerrier de Chiusi et une représentation de cavalier d'un lébès campanien conservés au British Museum, un vase attique du Louvre figurant un archer scythe coiffé d'un couvre-chef conique surmonté d'un protomé d'oiseau au long bec. Ce protomé peut également supporter un cimier comme l'attestent divers bronzes étrusques (voir l'exemplaire de la Bibliothèque nationale de Paris ; Born \& Hansen 1994, fig. 60 ; De Marinis \& Spadea 2004, 29) et grec (statuette d'Athéna Polias) et certaines peintures de vases grecs cf. Reinach, art. "Galea", Dict. ant. grecques et romaines, 1435, fig. 3415 et 3416

131- Ovide, Métamorphoses, 2.367-400.

132- Exemples dans Feugère 1994, 31, 64

133- Gaspari 2009. nifeste dans la production hellénistique dès la fin du $I V^{e} s$. a.C. et dont on retrouve la trace sur certaines émissions monétaires comme les tétradrachmes de Philippe V (casque ailé surmonté d'une tête de griffon).

Signalons, pour clore cette revue des casques à protomé d'oiseau, un dernier exemple. Il s'agit d'un petit bronze étrusque du ve s. a.C., découvert dans le sanctuaire vénète d'Altino, en Vénétie ${ }^{134}$ (fig. 65.1). La figurine, qui représente un archer agenouillé, a été identifiée à Pâris. Le personnage porte une tunique courte et un casque en forme de bonnet phrygien, figurant un rapace au plumage stylisé mais parfaitement reconnaissable. Le traitement naturaliste du casque, plus oiseau que casque, évoque un objet singulier du répertoire celtique de la seconde moitié du v $v^{e}$ s. a.C. Il s'agit d'un ornement figurant sur un bracelet de Reinheim (Allemagne), où l'on voit un personnage féminin aux bras repliés sur la poitrine avec des ailes d'oiseau (fig. 65.2) ; une palmette se substitue à la partie inférieure du corps tandis que la tête est coiffée d'un chapeau en forme d'oiseau à bec crochu ${ }^{135}$. Cette élaboration savante paraît construite sur le modèle de la Potnia theron (maitresse des animaux), illustrée au nord des Alpes par l'anse de l'hydrie grecque de Grächwil (Suisse), trouvée dans un tombeau hallstattien ${ }^{136}$ (fig. 65.3). Alors que l'exemplaire grec procède par juxtaposition, la version celtique privilégie nettement la fusion des éléments constitutifs du décor. Après avoir établi ce rapprochement, Stéphane Verger note que les couvre-chefs à décors animaliers d'Europe celtique relèvent de deux séries chronologiques distinctes : à une phase ancienne, allant du vI ${ }^{\mathrm{e}}$ s. a.C. au milieu du IV ${ }^{\mathrm{e}}$, caractérisée par des couvre-chefs/chapeaux, généralement associés à des personnages féminins, succède une période où les couvre-chefs agrémentés d'animaux ne sont plus de simples chapeaux ou coiffes, mais de véritables casques (serpent à tête de carnassier et cornes de bélier du protège-joue du casque d'Agris, rapace de Ciumesti, etc.).

L'évocation du casque de Cimesti, avec son volatile menaçant, nous amène à regarder de nouveau

134- Tirelli 2005, 301-316.

135- Verger 1991, 10, 16

136- Ibid. 
en direction des rives méditerranéennes où une série de représentations singulières a été mise en relation avec des mythes celtiques relatifs à l'intervention de la déesse des combats sous la forme d'une corneille. C'est à Raymond Bloch que l'on doit cette lecture ${ }^{137}$. L'exploit, qui valut au tribun militaire M. Valerius son surnom de Corv(in)us, en 349 a.C., à l'occasion du combat singulier qui l'opposa à un Gaulois de taille imposante (le corbeau attaque les yeux et le visage du Gaulois à coups de bec et de griffes), pourrait n'être selon lui qu'une simple transposition d'un mythe celtique, comme le supposait déjà $\mathrm{H}$. Hubert ${ }^{138}$. Denys d'Halicarnasse ${ }^{139}$ précise qu'après sa victoire M. Valerius porta toujours sur son casque un corbeau en guise d'emblème et que c'est ainsi que le représentèrent ceux qui le peignirent et sculptèrent. Si Rome n'a pas hésité à s'approprier le récit mythique de ses adversaires, leurs voisins étrusques l'illustrent, au contraire, toujours d'après R. Bloch, dans sa teneur originelle, puisque dans les représentations figurant sur certains sarcophages, l'oiseau n'attaque pas un Gaulois (généralement reconnaissable à leur nudité comme on peut le voir avec la celtomachie figurée sur le panneau principal de l'urne de Città della Pieve ${ }^{140}$ ) mais un guerrier armé à la manière grecque (l'oiseau monté sur le casque pointe le bec en direction des yeux) (fig. 72.1). Frappé au visage, le guerrier vacillant est sur le point de tomber. R. Bloch ne paraît pas s'étonner du contraste qui existe entre la scène du panneau central qui voit une victoire des Grecs sur les Celtes et celles latérales qui montre dans un cas l'aveuglement d'un Hellène sous les coups de "l'oiseau divin des Celtes" et dans l'autre, un guerrier identique au précédent se portant un coup d'épée au ventre (suicide d'Ajax). Si les casques celtiques supportent parfois un oiseau (on l'a vu avec le casque de Ciumesti, on l'a dit à propos de certaines figures du chaudron de Gundestrup et on l'a rappelé à travers les dires de Diodore), on ne connaît pas d'exemple d'oiseau frappant le possesseur du casque sur lequel il est juché. L'oiseau a pour fonction ordi-

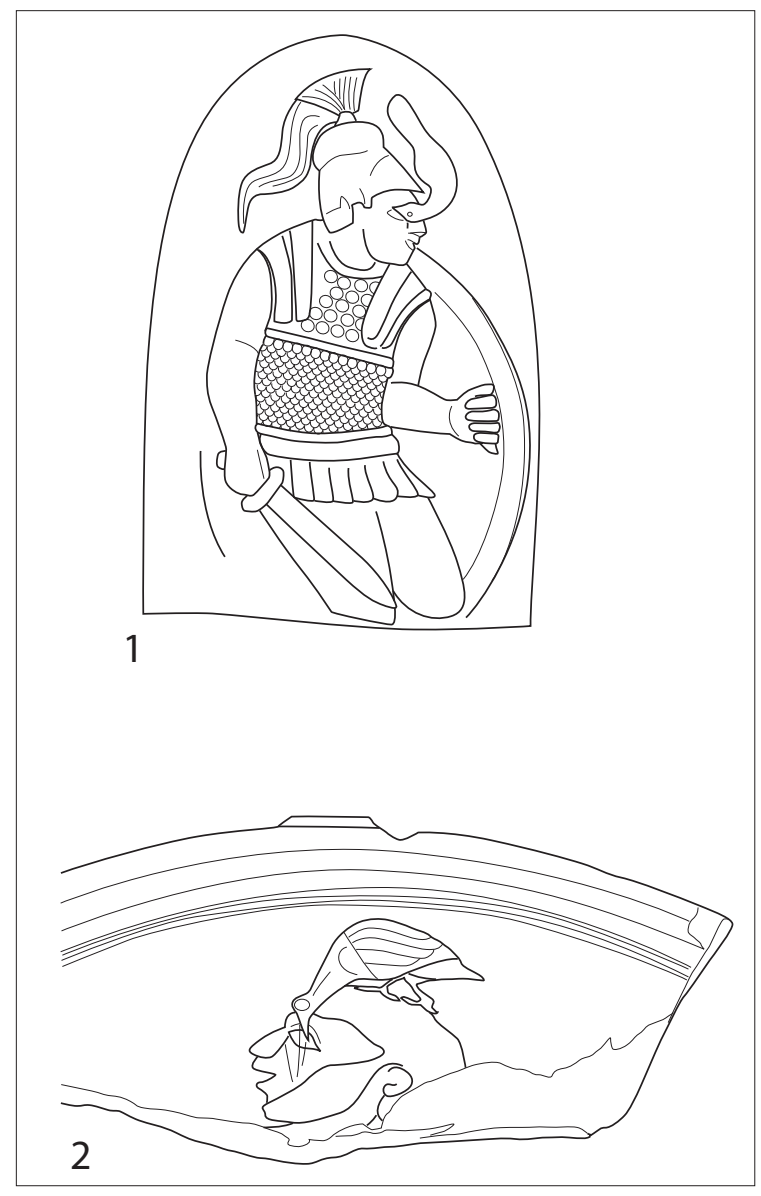

Fig. 72. Guerrier aveuglé par un oiseau perché sur son casque. 1. Urne étrusque de Città della Pieve, bas-relief latéral ( $d$ 'après Massa-Pairault 1985) ; 2. Megara Hyblea, fragment de vase à figure noire (Italie, d'après Vallet \& Villard 1964).

nairement d'effrayer l'adversaire. Faute d'exemple similaire, R. Bloch rejetait pour ce motif l'idée d'une influence grecque, tout en l'admettant de façon générale pour le décor des urnes et sarcophages étrusques d'époque hellénistique. C'était sans compter sur quelques découvertes inattendues comme l'a montré Mark I. Davies qui revient dans

137- Bloch 1966, 125-139; repris dans Peyre 1979, 107.

138. Hubert 1932, 38. C'est sous l'apparence d'un corbeau que la déesse Morrigu attaque le héros Cuchulainn.

139- Denys d'Halicarnasse, Ant. Rom., 15.1.

140- Massa-Pairault 1985, fig. 26 pour le panneau central, 27 et 28 pour les parties latérales. 
un bref article sur l'analyse de R. Bloch ${ }^{141}$. Ce nouvel élément nous est fourni par la cité grecque de Megara Hyblaea, en Sicile ${ }^{142}$. Il s'agit d'un fragment de vase à figure noire représentant la tête d'un guerrier coiffé d'un casque corinthien surmonté d'un oiseau en pied qui frappe de son bec le visage du personnage (fig. 72.2). Cet exemple daté du $\mathrm{VI}^{\mathrm{e}} \mathrm{s}$. a.C. montre qu'il n'est pas nécessaire de recourir aux mythes celtiques pour justifier le choix de ce motif par les sculpteurs étrusques. L'association de l'oiseau de proie à la guerre n'était certainement pas spécifique du monde gaulois, et leurs voisins ont pu développer des légendes plus ou moins analogues comme cela est parfaitement admis dans le cas, précédemment évoqué, des cygnes et des grues ${ }^{143}$.

Cette revue des casques et coiffures associés à un oiseau représenté en entier ou sous la forme de protomé nous a entraînés bien loin du cas particulier de Tintignac. Nous avons vu que ce type d'association, qui n'est pas rare, n'est nullement limité au monde celtique $^{144}$ et, qu'en dépit de nos recherches, la forme documentée à Tintignac ne trouve pas de parallèle direct ni ne vient se ranger dans une quelconque série. On ne saurait douter cependant des liens qui unissent, comme on l'a vu avec l'exemple du bracelet de Reinheim, les civilisations celtique et classiques. Pour autant, ces liens n'impliquent pas la dépendance de l'un par rapport à l'autre comme il semblait jadis ${ }^{145}$. Les artisans celtiques ont démontré durant toute la durée du second âge du Fer une indépendance d'esprit par rapport à ces modèles et ont développé une grammaire sans équivalent qui fait la spécificité de cet art. Les figures ornithomorphes rencontrées, rapaces et oiseaux aquatiques, entières et protomés, regardent toutes vers l'avant et se distinguent en cela de l'oiseau de Tintignac, qui

141- Davies 1979, 127-132, pl. 79-81

142- Vallet \& Villard 1964.

143- Davies 1979, 129, l'auteur rappelle que le thème du corbeau aveuglant un adversaire humain existait dans l'imaginaire grec bien avant l'époque hellénistique et cite à ce propos deux passages d'Aristophane tirés des Acharniens et des Oiseaux.

144- Chez les Daces, on peut signaler la phalère en argent de Surcea mentionné par M. Rusu dans son étude du casque de Ciumesti. La plaque figure un cavalier armé d'une épée (portée sur le flanc droit comme les Celtes) et d'un casque sur lequel se dresse un imposant oiseau - un rapace si l'on en juge par la forme crochue du bec - aux ailes déployées ; Rusu 1969, 285, fig. 8. 145- Déchelette 1914, 1508. rappelle à bien des égards les figures animales à tête renversée du début de La Tène. En vue frontale, et à la différence des autres exemplaires examinés, l'oiseau de Tintignac s'efface, ne laissant plus paraître qu'un prodigieux appendice cornu.

Dans cette étude nous avons essayé de mettre en évidence quelques-uns des traits qui font la spécificité de ce casque et de montrer, à travers l'examen de documents de différentes natures, son originalité. Pour autant, la question de la datation demeurent irrésolue dans la mesure où les comparaisons envisagées sont très diverses, tant par la datation que par la provenance, et que l'étude, en cours, du dépôt semble indiquer que l'on a réuni là des objets appartenant à différentes époques. Si la date d'enfouissement n'est pas antérieure à la seconde moitié du $\mathrm{I}^{\mathrm{er}} \mathrm{s}$. a.C., nous savons que certains objets datent de La Tène C2 (épées), d'autres de La Tène C1 (casques) et peut-être même de La Tène B (pour un des casques, semble-t-il). Si l'attribution du casque-oiseau à La Tène moyenne a notre préférence, cette proposition n'en est pas moins hypothétique. Les attendus concernant l'analyse du contenu sémantique de l'œuvre sont plus significatifs, du fait des liens que l'on peut établir par analogie avec les représentations figurées du début de la période de La Tène. Ce couvre-chef témoigne en effet de la permanence d'un langage graphique constitué de signes élémentaires qui se combinent pour former de nouvelles images. Le discours imagé passe par un brouillage des motifs originels grâce à un processus de transformation constante qui aboutit à leur désintégration et à leur recomposition, ici sous la forme d'un oiseau, peut-être un cygne, même si la figure représentée n'est assurément pas réaliste.

\section{LES TRAITEMENTS ET ÉTUDES}

\section{De la fouille à l'exposition : conservation et restauration (M. Drieux-Daguerre)}

Rares sont en France les interventions d'une seule équipe de restauration pour accompagner des objets de la fouille jusqu'à l'exposition, en assistant 
les études et la recherche ${ }^{146}$. Pourtant, si l'on admet l'importance de la préservation et de la mise en valeur des informations que renferme un objet archéologique, "véritable archive du sol", la conservation et la restauration s'imposent bien dès la fouille. En ce sens, les interventions du laboratoire de Materia Viva sur les pièces de Tintignac revêtent un caractère exceptionnel.

De la fouille en septembre 2004 à l'exposition du carnyx et du casque-oiseau en janvier 2009, l'équipe a développé des procédés novateurs au sein d'une chaîne logique de traitements, conçue selon les impératifs et les contraintes budgétaires et scientifiques de ce projet.

L'essentiel du dispositif a reposé sur la mise en place d'une suite d'interventions permettant de préserver le potentiel informatif de ces objets uniques.

Ainsi, la possibilité d'un prélèvement en bloc a été rapidement écartée en raison essentiellement de la fragmentation importante des objets. L'équipe de Materia Viva a alors été intégrée à la fouille et chargée in situ des mesures conservatoires : micro fouille, identification des matériaux, diagnostic de l'état de préservation, choix des modes de prélèvements, emballages, transport vers le laboratoire. Là, s'affine le diagnostic puis suivent l'enregistrement photographique des éléments et la radiographie, qui participent du constat. Enfin, un stockage provisoire dans des conditions climatiques stables a permis l'attente de décisions et la constitution d'une équipe de recherche.

Quatre ans après, la restauration du carnyx 467 et du casque-oiseau 468 atteste que les interventions premières ont bien permis de préserver les données tout au long des études et des examens.

146- Citons celles et ceux qui sont principalement intervenus dans ce projet, intégrés ou associés à Materia Viva :

-Interventions sur la fouille : Valérie Uzel, Monique DrieuxDaguerre, Tania Eyermann.

- Restaurations : Monique Drieux Daguerre, Valérie Uzel, Inocencia Queixalos.

- Radiographie : Céline Gargam et l'Apave de l'Union (MM Pontoni et Colas).

- Photographies : Materia Viva, Daniel Martin, Patrick Ernaux (INRAP).

-Supports, vitrines, exposition : Marc Jeanclos, Guillaume Blanchard et Éric Charpentier, atelier Jeanclos, Meaux.

\section{Préservation des données et prélèvements}

Lors de la découverte en 2004, les éléments du carnyx et le casque-oiseau, à l'instar de tous les alliages cuivreux du dépôt, présentent un bon état de préservation : les couches de corrosion sont fines et les formes des objets identifiables. La nature du sédiment, les structures bâties au-dessus de la fosse et la pente du terrain ont certainement contribué à créer un milieu d'enfouissement peu aéré et favorable à une dégradation lente des métaux. En outre, la corrosion des objets en fer déposés dans les niveaux supérieurs a été bénéfique aux alliages cuivreux dans les échanges électrochimiques.

La micro fouille constitue le premier constat d'état et le moment privilégié d'observation des matériaux. Il apparaît alors que les objets ont été démontés ou fragmentés avant leur dépôt dans la fosse : les cassures et les marques de frappes volontaires présentent une couche de corrosion similaire à celle des surfaces. De même, on note rapidement l'absence quasi-totale de restes de matériaux organiques : pas de jugulaires, pas de restes de textiles ni de cuir pouvant être associés aux casques. Or les sels de cuivre agissent généralement en biocides et de façon favorable dans la préservation de ces matières. En revanche, les restes de brasure ou d'étain sont nombreux, apparaissant dès la fouille sous la forme de concrétions grisâtres dures et fissurées ; plusieurs sont notées sur le pavillon du carnyx, à l'intérieur du casque-oiseau et autour des yeux des animaux. Enfin, la présence d'une pupille noire en lignite, témoin ténu et fragile, incite à la prudence et à la mise en œuvre de prélèvements particuliers.

Le dégagement du carnyx et du casque-oiseau intervient alors que toutes les autres pièces ont déjà été prélevées. Les deux objets reposent face gauche contre terre; quelques fragments des autres objets déjà enlevés, soudés par la corrosion en surface des deux pièces, sont laissés en place (fragments du casque à anneaux, lames de fer...) ainsi que le sédiment de remplissage. Leur dégagement est différé en laboratoire, avec des moyens d'observation plus précis.

La consolidation des tôles fines fissurées ou fracturées et de tous les matériaux connexes fragiles est effectuée in situ avec une résine acrylique diluée dans de l'eau déminéralisée (Primal AC33). 
Mais c'est l'utilisation du cyclododécane pour composer des chapes de prélèvement qui était en 2004 novatrice. Issu de la chimie de synthèse dès les années 30, ce produit est employé dès 1995 en Allemagne, puis en Suisse pour la conservation des collections archéologiques ${ }^{147}$. Une restauratrice de Materia Viva, Tania Eyermann, l'avait utilisé en 2001 à Münich ${ }^{148}$.

Cet hydrocarbure cyclique - de composition $\mathrm{C}_{12} \mathrm{H}_{24}$ - a la propriété d'être hydrophobe, de pouvoir être appliqué sous forme liquide et à chaud, de refroidir rapidement, puis de se sublimer lentement à l'air ambiant ; il est en outre radio transparent et n'entrave ainsi pas les radiographies ${ }^{149}$. Il permet de constituer des coques temporaires semi-rigides et légères, bien moins pesantes que les chapes de plâtres ; il s'élimine à l'air ambiant ou par air chauffé, c'est-à- dire sans contrainte physique. Hydrophobe, sa pénétration dans les matériaux est freinée par l'humidité résiduelle lors de la fouille, et les analyses ultérieures ne semblent pas modifiées. Enfin, la prise immédiate réduit les durées de prélèvements et ne ralentit pas la fouille outre mesure. Face au peu de documentation sur les risques liés à son emploi, on veille à travailler en atmosphère ventilée. Posé sur les faces visibles des objets et sur une gaze de coton servant d'armature, le cyclododécane s'est révélé essentiel pour préserver les tôles fines et les éléments fragilisés du carnyx et du casque-oiseau (fig. 73). Ainsi prélevés, puis emballés dans du film étirable de polyéthylène et bloqués pour le transport par des chips de polystyrène dans des bacs gerbables, les objets sont dirigés vers Toulouse, puis au cabinet de radiographie.

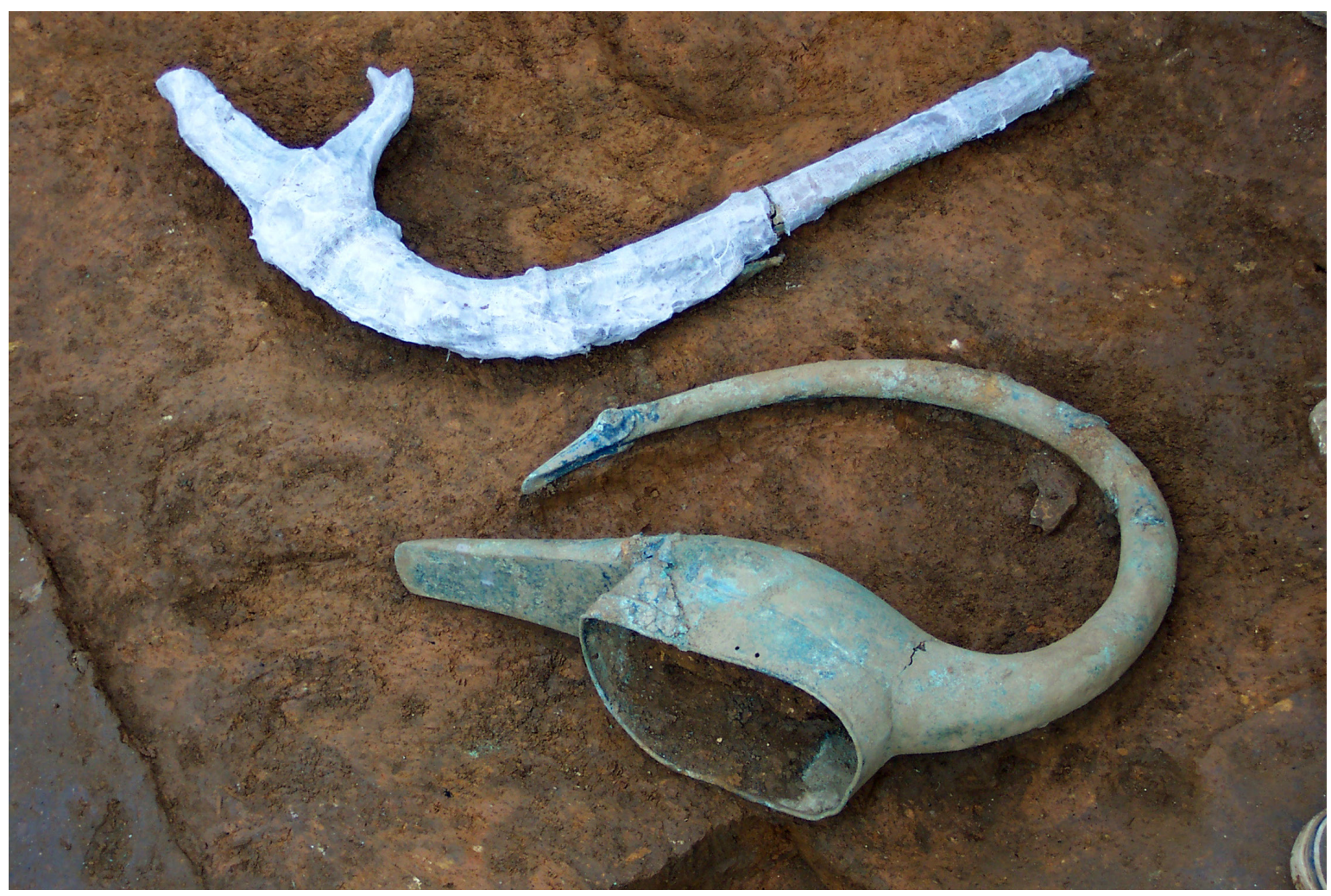

Fig. 73. Micro fouille du casque-oiseau et prélèvement du carnyx à l'aide de cyclododécane (cl. P. Ernaux, INRAP).

147- Hangleiter \& Jägers 1995, 385-392.

148. Eyerman 2001.

149. Tissier 2007. 


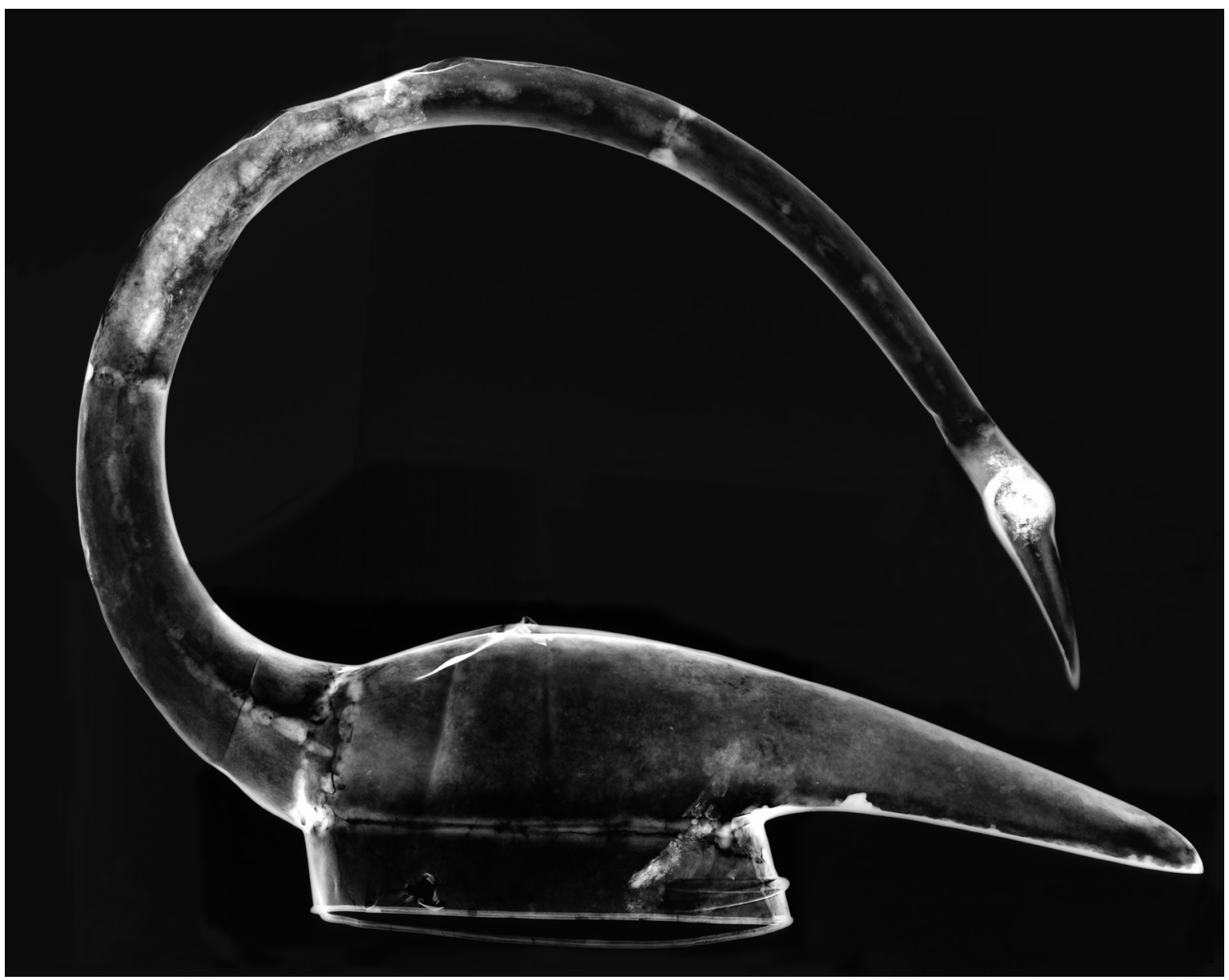

Fig. 74. Cliché radiographique du casque 468 montrant les différentes brasures et sections (cl. C. Gargam et Apave-I'Union).

L'examen aux rayons $\mathrm{X}^{150}$ a livré rapidement des informations essentielles à une première étude des deux objets : la présence des brasures reliant les nombreux éléments qui composent le casque-oiseau (fig. 74); celle d'un alliage dense autour des yeux des objets et des perforations des oreilles du carnyx ; la présence d'un tube dans le pavillon du carnyx qui n'a pu être extrait et pour lequel la radiographie constitue le seul moyen d'étude ; enfin, les nombreux détails technologiques.

150- Radiographies : Céline Gargam Materia Viva puis Aktis Adélos, et MM Pontoni et Colas (Apave 31).
Les photographies complètent le constat d'état avant toute intervention; elles sont réalisées par Materia Viva et par Patrick Ernaux (INRAP).

\section{La mise en attente : un nouveau mode de stockage}

Dans l'attente des crédits de restauration et d'étude, il nous est demandé début 2005 de concevoir un emballage mettant les pièces à l'abri de la poussière et des variations climatiques, limitant les manipulations et les photographies, tout en laissant visibles les moindres fragments. Le sédiment se rétractant au séchage, son élimination est décidée 
avant tout emballage. Le traitement des 469 pièces se déroule de janvier à août 2005.

Pour garantir la conservation de moyen terme, les matériaux employés pour le stockage sont tous chimiquement neutres, essentiellement composés de polyéthylène.

Etant donné les dimensions des objets fragmentés, le module de base retenu est le bac gerbable de type Alibert ${ }^{\circledR}$. Le casque-oiseau, hors dimensions standard, est emballé dans une boîte confectionnée sur mesure dans du polypropylène cannelé.

Dans chaque bac, les éléments sont disposés sur des plateaux de mousse Plastazote ${ }^{\circledR}$ (polyéthylène de cellules fermées fabriqué sous azote, sans oxygène) et isolés des parois par une feuille de Tyvek lisse ${ }^{\circledR}$ (polyéthylène).

Chaque plateau est lui-même placé dans une enveloppe composée de deux types de films multicouches de polyéthylène (partie supérieure) et de polyéthylène associé à de l'aluminium (partie inférieure) ${ }^{151}$; l'enveloppe est scellée à $190^{\circ} \mathrm{C}$ sous 3 bars et pendant 3 secondes. Ces matériaux sont em- ployés en 2004 pour la mise en anoxie des collections ethnographiques du Museum d'histoire naturelle de Toulouse ${ }^{152}$. L'élimination de l'oxygène - qui serait idéale pour la conservation à long terme de métaux - n'est pas retenue : plusieurs collègues nous signalent des augmentations importantes d'humidité relative dans les enceintes créées lors du fonctionnement des absorbeurs d'oxygène employés à l'époque. Un vide partiel simple est effectué pour limiter la quantité d'air autour des objets (fig. 75).

Ce mode d'emballage s'est révélé efficace au-delà de la durée préconisée par le fabricant, soit une année. En effet, depuis 2005, les barrettes témoins d'humidité relative placées sur des plateaux conservés en atmosphère sèche présentent toujours aujourd'hui moins de $20 \%$ d'HR. On peut expliquer cette stabilité par le fait que les plateaux n'ont pas été manipulés, et qu'aucune fuite d'air n'a eu lieu. Mais aussi, la conservation depuis 2004 de toute la collection dans une pièce à humidité contrôlée a certainement maintenu des conditions générales stables.

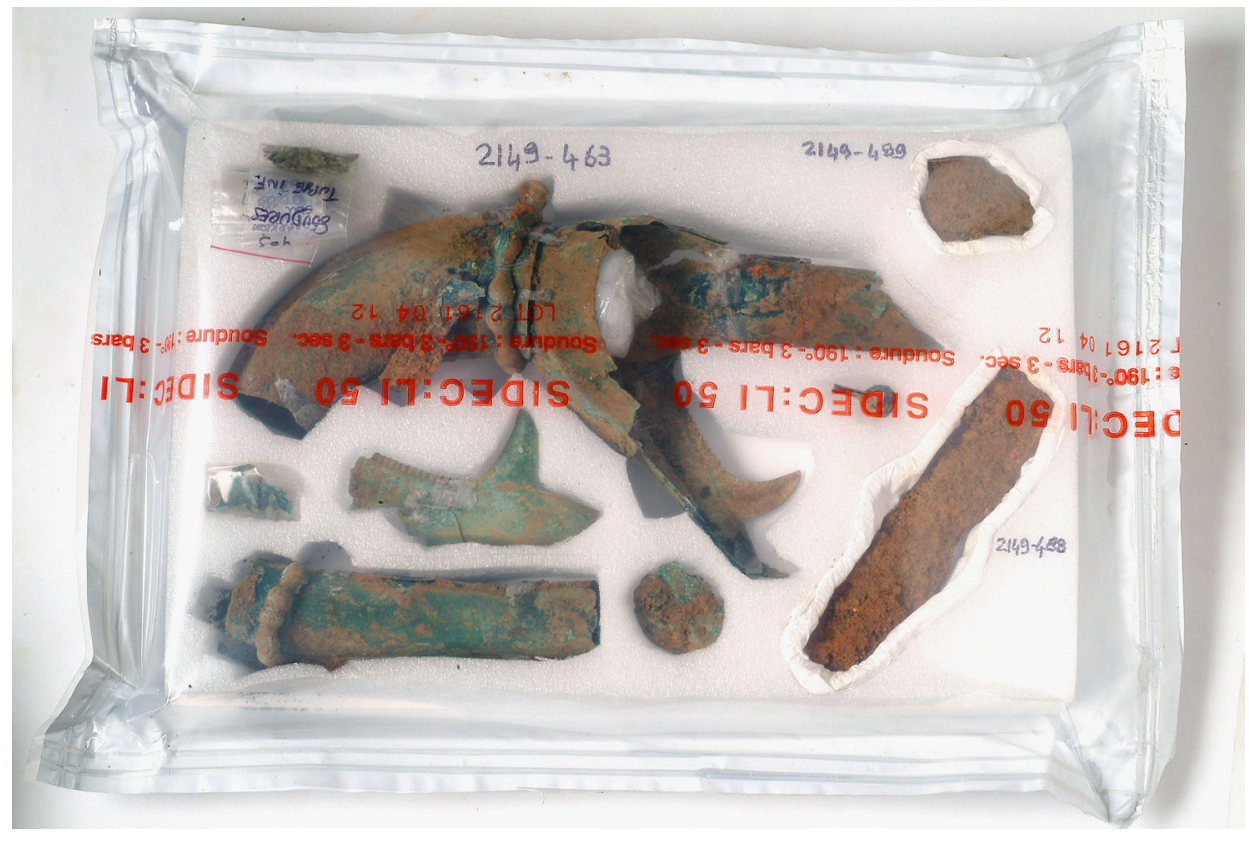

Fig. 75. Mise en atmosphère neutre et stable d'éléments du dépôt (cl. Materia Viva). 


\section{La restauration des deux pièces}

Déjà bien documentés par les examens et les études antérieurs, le casque-oiseau et le carnyx sont restaurés en $2008^{153}$ pour être exposés de 2009 à 2010 dans quatre lieux différents.

Afin de limiter les risques inhérents aux déplacements, Materia Viva accueille différents acteurs et met à leur disposition les espaces et moyens nécessaires : chercheurs, métallographes, dessinateurs, infographistes, commissions scientifiques, tous ont pu consulter ou étudier avec autorisation les collections dans des conditions de conservation satisfaisantes.

La restauration a débuté par la reconstitution des deux objets et la recherche de fragments complémentaires; les relevés et les photographies pris sur le terrain se sont révélés précieux.

Les interventions sont particulièrement suivies par Barbara Armbruster pour valider le dégagement des éléments technologiques. Ainsi, sous la couche fine mais indurée des produits de corrosion, éliminée par voie mécanique ou par gels chimiques, apparaissent de nombreux détails ténus : les traces de polissage sur le timbre du casque-oiseau ; les marques des brasures diffusées dans le métal ; des croix imprimées dans les motifs de colliers de serrage du carnyx ; les traces de martelage au revers des tôles.... Le dégagement des surfaces et des produits de corrosion, la consolidation des zones fragiles, la protection par pose de résines adaptées ${ }^{154}$, constituent une tâche de longue haleine : près de cent soixante heures pour le carnyx, environ quatre-vingts pour le casque-oiseau.

Mais la restitution n'aurait pu se faire sans l'intervention de l'atelier Jeanclos ${ }^{155}$. Une des prouesses est de présenter le carnyx à la verticale, pour mieux évoquer sa fonction.

Les contraintes imposées à l'atelier Jeanclos sont de concevoir des supports en acier gainé de polyéthylène, rigides et légers, qui puissent garantir la conser-

153- Restauration du carnyx : Inocencia Queixalos ; restauration du casque-oiseau : Monique Drieux-Daguerre - avec l'assistance de l'équipe de Materia Viva.

154- Réduction ponctuelle des chlorures de cuivre par oxyde d'argent - protection Incral.

155- Marc Jeanclos, Guillaume Blanchard, Éric Charpentier : atelier Jeanclos, Meaux (77). vation des deux objets, de faciliter la présentation et de réduire les manipulations directes.

Le support du carnyx réalisé est en fait intimement lié à l'objet, tout en étant réversible : il est composé de tiges passant à l'intérieur de chaque fragment et montées sur des blocs-supports qui s'encastrent les uns aux autres en un montage savant et solide.

Le support du casque-oiseau épouse et soutient la forme complexe par l'intérieur.

Dans les deux cas, la conception de l'atelier Jeanclos s'est orientée vers une mise en valeur des pièces, les supports disparaissant au profit des œuvres (fig. 76).

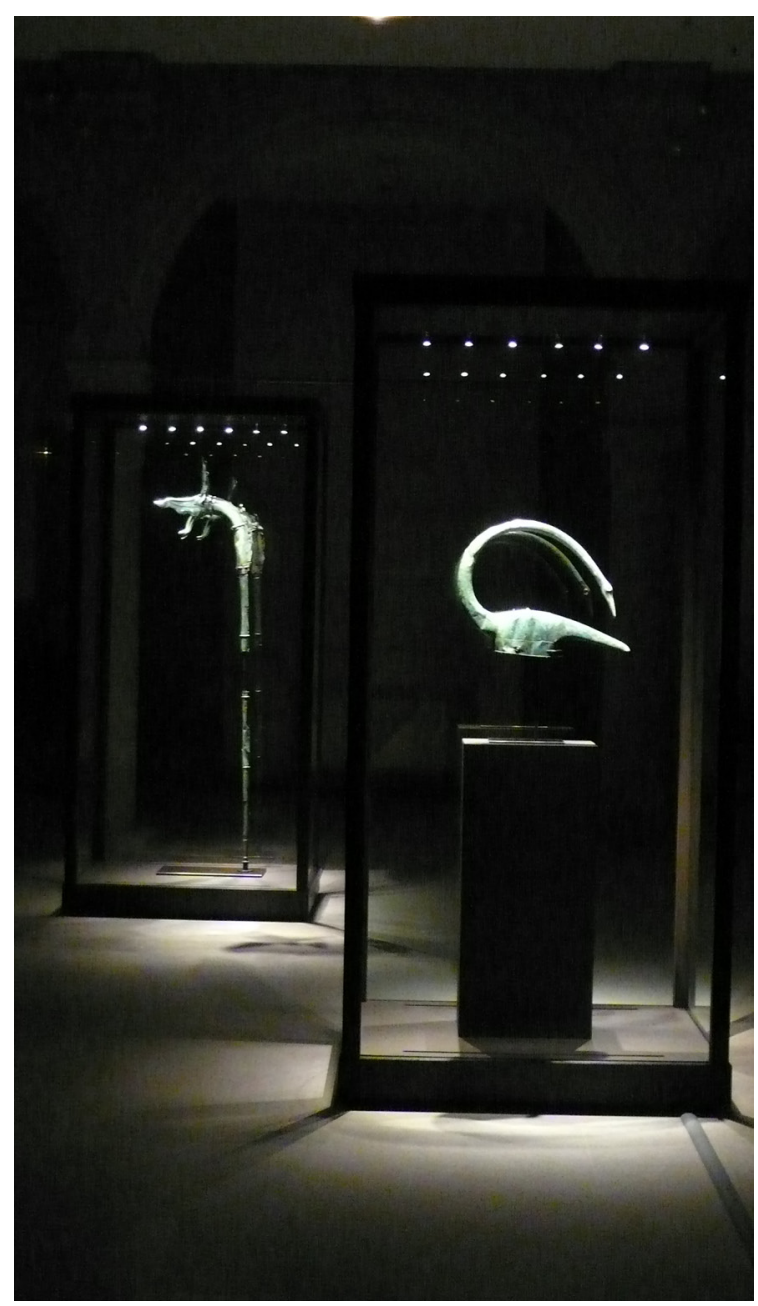

Fig. 76. Mise en espace du carnyx et du casque-oiseau à Tulle en 2009 (cl. Materia Viva). 
Les vitrines ont été conçues et réalisées par le même atelier pour compléter la réflexion et intégrer les supports : verres Sécurit, réserves de produits de dessiccation, éclairage interne, sobriété des lignes. L'atelier Jeanclos est associé à Materia Viva pour le montage et le démontage des deux pièces.

De la fouille à l'exposition, Materia Viva a pu ainsi garantir la préservation des données et leur étude, la conservation de l'ensemble de la collection, la restauration et l'exposition du carnyx et du casque-oiseau. La conservation-restauration a été conçue comme le pivot essentiel de la chaîne d'interventions, offrant aux différents acteurs les éléments d'étude et de compréhension. L'application de procédés nouveaux pour le prélèvement et la conservation des objets garantit, six ans après la découverte, la préservation du potentiel informatif de cette collection.

\section{La numérisation 3D des objets du dépôt de Tintignac \\ (L. Espinasse, P. Mora [ArchéoTransfert])}

Afin de faciliter l'étude et de garder le maximum d'informations sur l'aspect des deux premiers objets du dépôt de Tintignac avant leur restauration, l'équipe scientifique a décidé d'en réaliser la numérisation en trois dimensions.

Plusieurs techniques de numérisation tridimensionnelle existent; nous avons opté pour un scanner utilisant un balayage laser. La source lumineuse est donc constituée par la raie du faisceau laser projetée sur l'objet, une caméra numérique intégrée permet l'analyse du signal lumineux et le calcul par triangulation de la distance scanner/objet.

Aucun contact physique n'est nécessaire, ce qui est particulièrement appréciable dans le cas d'objets ne supportant pas les manipulations répétées comme cela est le cas ici.

L'appareil utilisé est constitué de deux parties, la tête du scanner comprenant le système optique d'acquisition, et un bras mécanique possédant à chacune de ses articulations un codeur électronique qui renseigne la tête d'acquisition sur sa position dans l'espace. La numérisation proprement dite se fait en balayant l'objet à l'aide du faisceau laser par passes successives (fig. 77). Grâce aux informations recueillies par le bras, chaque nouvelle acquisition de

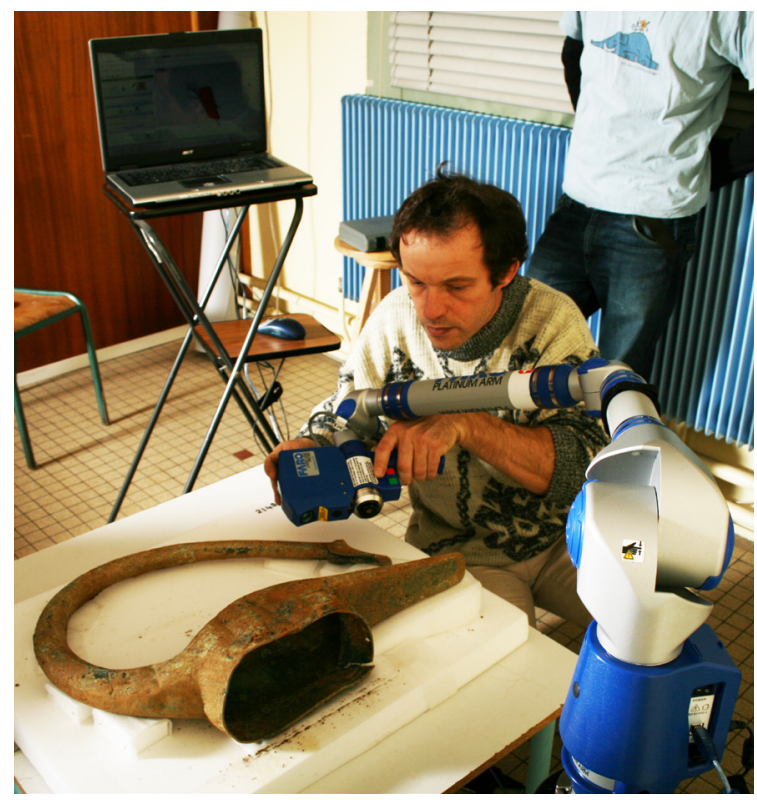

Fig. 77. Utilisation du scanner à balayage laser sur le casqueoiseau (Archéotransfert).

données est automatiquement en connexion avec la précédente tant que l'appareil ou l'objet n'est pas déplacé.

Avec ce procédé, ce sont environ 19000 points par seconde qui sont mesurés à la surface de l'objet. Ces points sont définis dans les trois dimensions de l'espace (X, Y, Z). Le résultat est un véritable "nuage de points" plus ou moins dense en fonction de la vitesse de déplacement du scanner. Le logiciel de pilotage sert alors de guide pour visualiser le résultat en temps réel, ce qui évite de laisser des zones non numérisées. La résolution finale (distance entre deux points mesurés à la surface de l'objet), dans le cas des objets de Tintignac, reste inférieure au millimètre, ce qui représente plusieurs centaines de milliers à plusieurs millions de points de mesure par objet. La précision est inférieure au $1 / 10^{\mathrm{e}}$ de millimètre.

Une fois la numérisation effectuée les nuages de points peuvent être maillés, c'est-à-dire qu'une surface constituée de triangles est calculée à partir de ces points (fig. 78). Cette surface permet de donner un aspect plus physique à l'objet de manière à être 


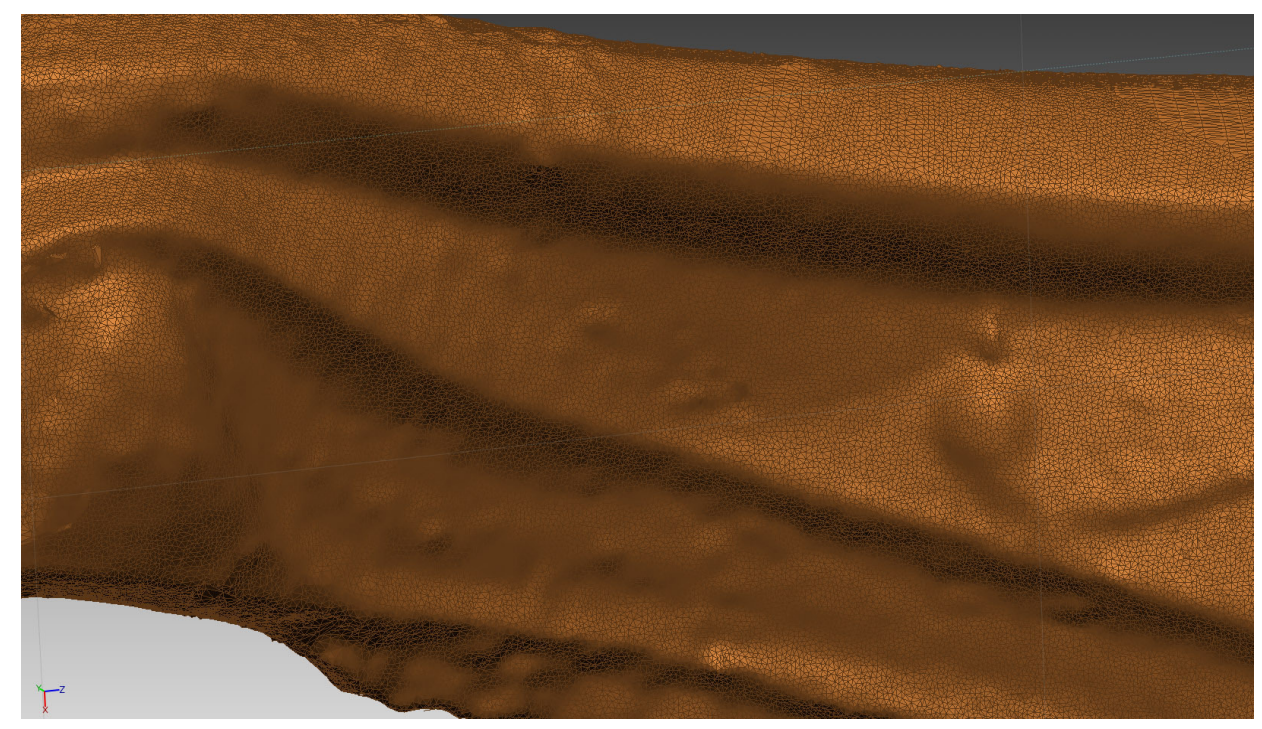

Fig. 78. Une surface constituée de triangles est calculée à partir des points mesurés. Ici une portion du pavillon du carnyx (Archéotransfert).

mieux interprété visuellement et à pouvoir être exporté, visionné ou traité dans d'autres logiciels. Lobjet numérique 3D ainsi obtenu constitue donc une sorte de double numérique tridimensionnel de l'objet réel et il permet, comme ce dernier, la réalisation d'un certain nombre de mesures et de calculs difficilement réalisables sans risques sur les objets originaux (fig. 79 et 80). Ainsi des calculs de volume, surface ou encore d'épaisseur peuvent être effectués sur le modèle numérique à l'aide de logiciels appropriés (fig. 81).

Le modèle numérique 3D peut aussi servir de base à une restitution 3D numérique de l'objet originel. Dans ce cas il faudra remodéliser les objets en restant au plus près des données fournies par le scanner mais en "gommant" les altérations et autres outrages du temps subis par les objets. Dans certains cas, des parties manquantes pourront être restituées à partir des fragments existants ou bien en prenant pour modèle d'autres objets similaires du dépôt. En plus de la géométrie, les matériaux peuvent également être restitués. Il est ainsi possible de redonner l'aspect originel du métal (doré ou argenté) et de restituer virtuellement l'apparence probable des objets à l'époque où ils étaient encore utilisés.

Le modèle numérique constitue donc également un excellent support pour tester et faire partager les hypothèses de restitutions proposées par les archéologues. 
Fig. 79. Modèle numérique 3D du casque-oiseau constitué par un nuage de plus de 2,6 millions de points montrant la géométrie du casque avant sa restauration. La couleur de l'objet est artificielle car elle n'est pas capturée par le scanner 3D ; ce mode de visualisation peut permettre la révélation de détails masqués par la couleur réelle de l'objet

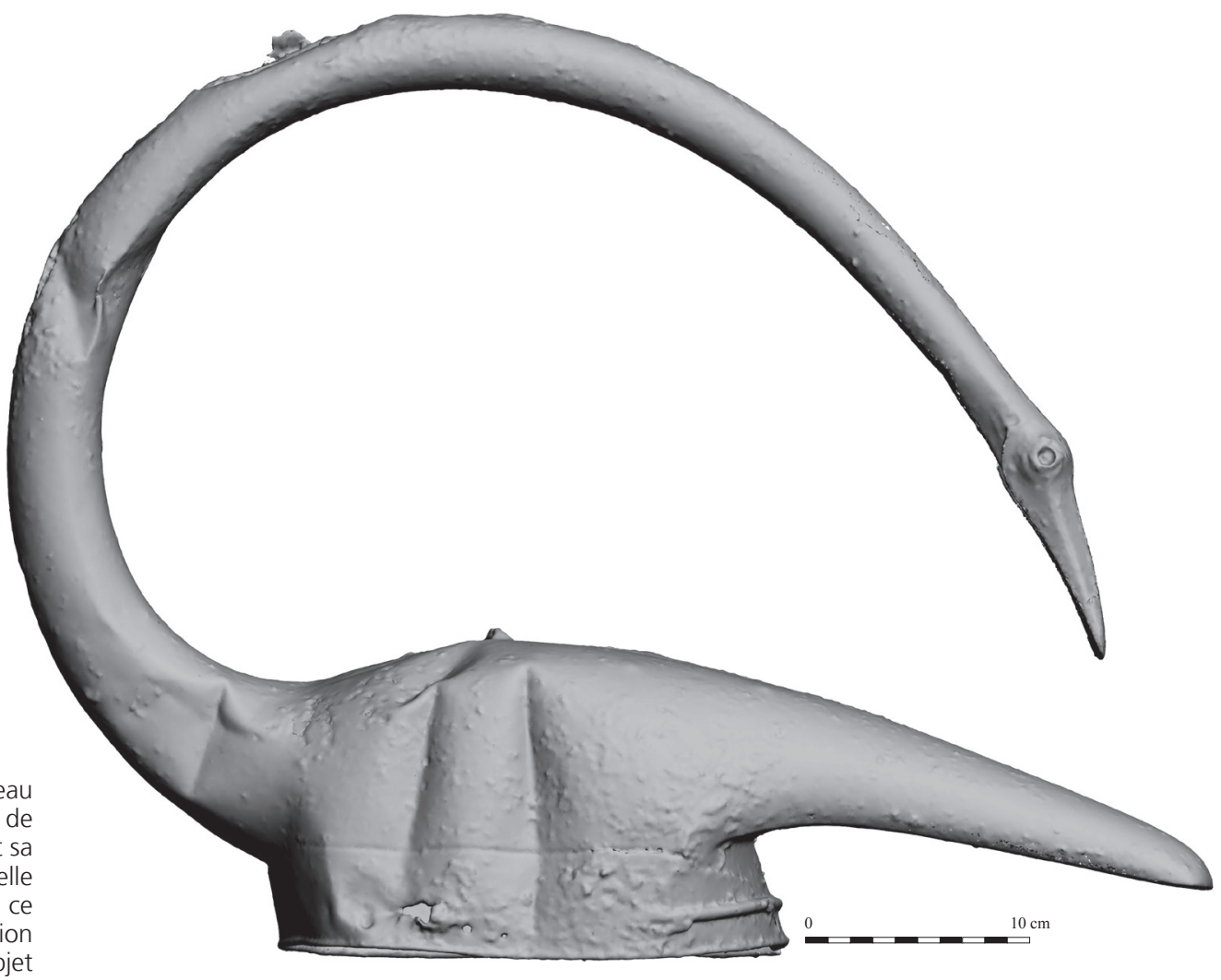
(Archéotransfert).

Fig. 80. Modèle numérique 3D monochrome du pavillon d'un carnyx réalisé avant la restauration. Le nuage est constitué de plus de 1,5 millions de points espacés chacun de moins d'un millimètre (Archéotransfert).

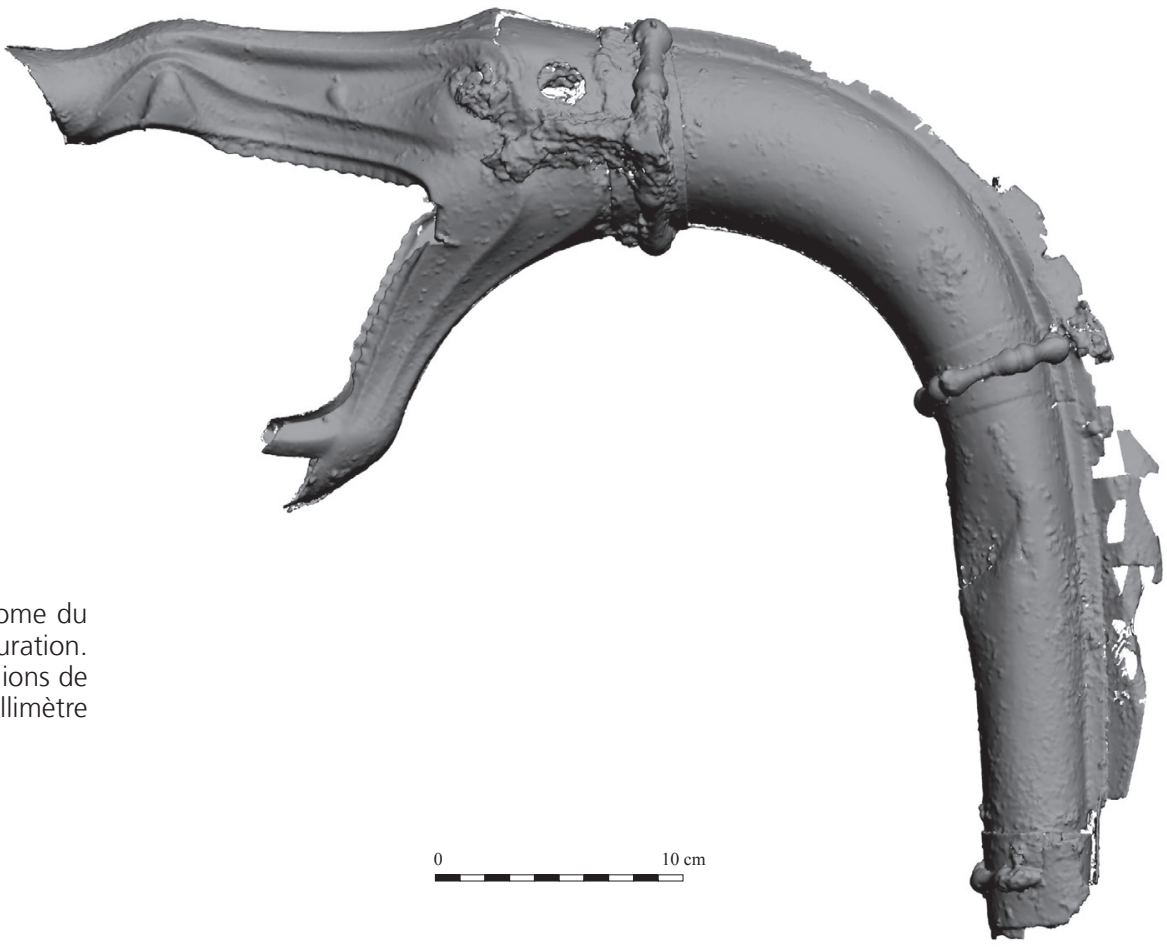




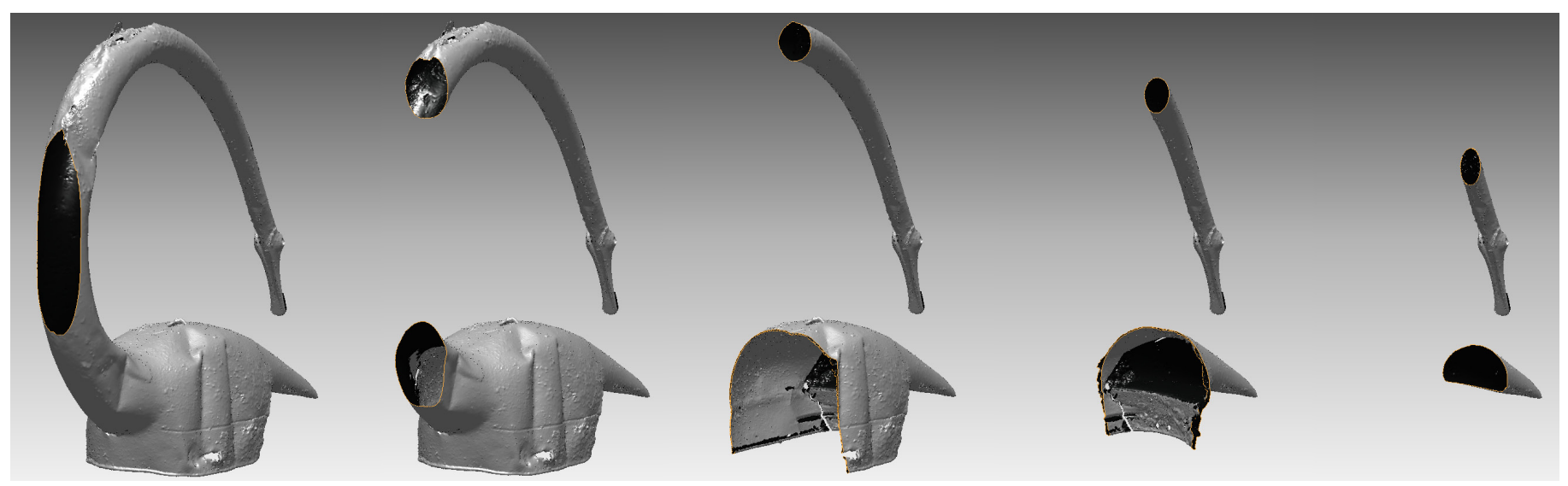

Fig. 81. Les nuages de points permettent, entre autres, la réalisation de coupes à différents niveaux de l'objet. Ces mesures seraient très délicates à faire avec autant de précision sur l'objet lui-même (Archéotransfert).

\section{Bibliographie}

\section{ABRÉVIATIONS \\ LT : de La Tour \& Fischer 1999. \\ DT : Delestrée \& Tache 2007.}

Adamski, F. (2008) : "Ateliers, techniques et artisans du 'bronze' en Aquitaine antique, de la fin de l'âge du fer à la période gallo-romaine", doctorat de I'Université Michel de Montaigne de Bordeaux 3.

Amy, R., P.-M. Duval, J. Formigé, J.-J. Hatt, A. Piganiol, Ch. Picard et G.-Ch. Picard (1962) : L'arc d'Orange, Gallia Suppl. 15, Paris.

Au temps des Celtes (1986) : Au temps des Celtes, ve - er siècle avant J.-C., Daoulas.

Barral, Ph., G. Bossuet, G. Kuhnle, J.-Y. Marc, P. Mougin, D. Arcay, G. Bataille, S. Blin, A.-S. Bride, A. Burgevin, C. Camerlynck, M. Dabas, A. Dumont, B. Fort, J.-P. Guillaumet, L. Jaccotey, L. Jeunot, M. Joly, E. Marmet, J.-P. Mazimann, S. Mouton, V. Pichot, M. Schönfelder, M. Thivet et B. Vannière (2007) : "Epomanduodurum, une ville chez les Séquanes. Bilan de quatre années de recherches sur l'agglomération antique de Mandeure - Mathay (Doubs)", Gallia, 64, 353-434.
Barral, Ph. (2009) : "Les dépôts du sanctuaire de Mandeure", in : Le site de La Tène : bilan des connaissances, état de la question, Actes de la Table ronde internationale de Neuchâtel, 1-3 novembre 2007, Archéologie neuchâteloise 43, Neuchâtel, 185-195.

Bertrand, I. et P. Maguer (2007) : De pierre et de terre. Les Gaulois entre Loire et Dordogne, Catalogue de l'exposition présentée par les musées de la Ville de Chauvigny (Vienne) du 15 mai au 14 octobre 2007, Pont-l'Abbé.

Bertrand, I., A. Duval, J. Gomez de Soto et P. Maguer (2009) : Les Gaulois entre Loire et Dordogne, Actes du XXXle colloque international de l'AFEAF, 17-20 mai 2007, Chauvigny (Vienne), Mémoire 34.

Bloch, R. (1966) : Traditions celtiques dans I'histoire des premiers siècles de Rome, in : Mélanges d'archéologie, d'épigraphie et d'histoire offerts à Jérôme Carcopino, Paris, 125-139.

Boekhoorn, D. N. (2008) : Bestiaire mythique, légendaire et merveilleux dans la tradition celtique: de la littérature orale à la littérature écrite, Université Rennes 2, University College Cork, thèse de Doctorat.

Bohn, R. (1885) : Das Heiligtum der Athena Polias Nikephoros, Altertümer von Pergamon $\mathrm{Bd} 2$, Berlin, 2 vol.

Bois, G. (1999) : La ciselure et ses techniques, Paris. 
Born, H. et S. Hansen (1994) : Frühgriechische Bronzehelme, Bd. III, Sammlung Axel Guttmann, Mayence.

Briard, J. et A. Duval, éd. (1993) : Les représentations humaines $d u$ néolithique à l'âge $d u$ Fer, Actes du $115^{e}$ congrès national des sociétés savantes, Paris.

Buchsenchutz, O., dir. (2008) : "Les Celtes", Les dossiers d'archéologie, 329,

Charpy, J.-J. et A. Rapin (1991) : "La sépulture celtique de Marson "Montfercault " (Marne)", Bulletin de la Société d'Agriculture, Commerce, Sciences et Arts de la Marne, CVI, 7-23.

Clodoré, T. (2002) : "L'introduction du métal dans le paysage sonore : de l'âge du Bronze à la fin du premier âge du Fer (2300-450 avant J.-C.)", in : Préhistoire de la musique, Sons et instruments de musique des âges du Bronze et du Fer en France, Catalogue d'exposition présentée au musée de Préhistoire d'lle-de-France de Nemours, du 17 mars au 10 novembre 2002, Argenton-sur-Creuse.

Crawford, M. H. (1974) : Roman Republican Coinage, Cambridge, vol. I et II.

Das Rätsel der Kelten (2002) : Das Rätsel der Kelten. Glaube, Mythos, Wirklichkeit, eine Austellung des Landes Hessen in der Schirn Kunsthalle Frankfurt, mai - septembre 2002, Stuttgart.

Davies, M. I. (1979) : " L'oiseau sur le casque : le corbeau divin des Celtes, M. Valérius Corv(in)us, et Tite-Live 7,26", in : Bronzes hellénistiques et romains, tradition et renouveau, Cahiers d'Archéologie Romande 17, Lausanne, 127-132.

De La Tour, H. et B. Fischer (1999) : Atlas des monnaies gauloises, 3e éd., Paris.

De Marinis, R. (1997) : "La tomba gallica di Castiglione delle Stiviere (Mantova)", Notizie Archeologiche Bergomensi, 5, 115-177.

De Marinis, R.C. et G. Spadea (2004) : I Liguri. Un antico popolo europeo tra Alpi e Mediterraneo, Milan-Gènes.

De Navarro, J. M. (1972) : The Finds from the site of La Tène, I. Scabbards and the Swords found in them, Londres, 2 vol.

Déchelette, J. (1914) : Manuel d'archéologie préhistorique, celtique et gallo-romaine, t. II : Archéologie celtique ou protohistorique, Troisième partie, Second âge du Fer ou époque de La Tène, Paris.

Delestrée, L.-P. (1980) : "Les monnaies 'au coq' frappées en Gaule Belgique", Revue numismatique, 6e série, t. 22, 33 62.

Delestrée, L.-P. et M. Tache (2007) - Nouvel atlas des monnaies gauloises, III. La Celtique, du Jura et des Alpes à la façade atlantique, Saint-Germain-en-Laye.

Depeyrot, G. (2004) : Le numéraire celtique, III. de l'Atlantique aux Arvernes, Wetteren, Moneta 36, 2004.

Deyber, A. (2009) : Les Gaulois en guerre - Stratégies, tactiques et techniques - Essai d'histoire militaire $\left(\|^{e}-\right.$ er $^{r}$ siècle av. J.C.), Paris.

Ducrey, P. (1985) : Guerre et guerriers dans la Grèce antique, Paris.

Durantel, P. (2007) : Le sanglier et ses chasses, Chamalières.

Duval, P.-M. (1977) : Les Celtes, coll. L'Univers des Formes, Paris.
Duval, A. (1982/83) : "Note sur un vase de La Tène à décor ornithomorphe", Antiquités Nationales, 14/15, 64-69.

Eyermann T. (2001) : Traitement de deux mottes prélevées insitu, emploi du cyclododécane, Mémoire de Master de conservation des biens culturel, Université de Paris I.

Farkas, P. (1981) : L'art de jouer les Cuivres, Paris.

Feugère M. (1993) : Les armes des Romains, de la République à I'Antiquité tardive, Paris.

- (1994) : Casques antiques, Paris.

Fogolari, G. (1960) : "Sanzeno nella Anaunia", in : Civiltà del Ferro. Studi pubblicati nella ricorrenza centenaria della scoperta di Villanova, Bologne, 267-321.

Frey, O. H. (2008a) : "Homme ou héros ? Les statues du Glauberg et la grande sculpture chez les Celtes anciens", in : Buchsenschutz, dir. 2008, 64-71.

- (2008b) : "Le premier art celtique. Dieux et demons", in : Buchsenschutz, dir. 2008, 48-63.

Gaspari, A. (2009) : "Some Iron Age and Early Roman finds from Stari grad above Unec (Notranjska, Slovenia)", in : Tiefengraber et al., éd. 2009, 315-329.

Geneviève, V. (2007) : "Catalogue des monnaies", in : HanryBesombes 2007, 138-139.

Gilbert, J. (2000) : http://perso.univ-lemans.fr/ jgilbert/ ArticlesPageWeb/LesCuivres.pdf

Grunwald, S., J. K. Koch, D. Mölders, U. Sommer et S. Wolfram (2009) : Artefact, Festschrift für Sabine Rieckhoff zum 65. Geburtstag, Universitätsforschungen zur prähistorischen Archäologie Band 172, Bonn.

Guggisberg, M. A. (2009) : "Les grandes Oreilles", L'Archéologue, 103, 47.

Guichard, V. et Fr. Perrin, dir. (2002) : L'aristocratie celte à la fin de l'âge du Fer (IIe s. avant J.-C. - er S. après J.-C.), Actes de la table ronde, Glux-en-Glenne, 10-11 juin 1999, Centre européen d'archéologie du Mont Beuvray, Glux-en-Glenne.

Hangleiter H. M. et E. Jägers (1995) : "Flüchtige Bindemittel", Zeitschrift für Kunsttechnologie und Konservierung, 2, 385-392.

Hanry-Besombes, A. (2007) : Malemort/corrèze. L'occupation laténienne et gallo-romaine à la confluence de la Corrèze et de la Couze (bassin de Brive), RFO de fouille préventive, INRAP.

Hautenauve, H. (2001) : Catalogue $n^{\circ} 82$, "Anneau de Mérysur-Seine", in : Moret et al. 2001, 152.

Hickmann E., I. Laufs et R. Eichmann, dir. (2000) : Studien zur Musikarchäologie II, Musikarchäologie der frühen Metallzeiten der Welt, Orient-Archäologie 7, Rahden/ Westf.

Hubert, H. (1932) : Les Celtes depuis l'époque de La Tène et la civilisation celtique, coll. L'évolution de l'humanité, Paris.

Hunter, Fr. (2001) : "The carnyx in Iron Age Europe", The Antiquaries Journal, 81, Londres, 77-108.

- (2009a) : "Une oreille de carnyx découverte à La Tène", in : Le site de La Tène : bilan des connaissances - état de la question, Actes de la Table ronde internationale de Neuchâtel, 1-3 novembre 2007, Archéologie neuchâteloise $43,75-85$. 
- (2009b) : "The carnyx and other trumpets on Celtic coins", in : Coinage in the Iron Age - essays in honour of Simone Scheers, Londres, 231-248.

- (2009c) : "L'étrange casque d'un petit cheval, le chanfrein de Torrs", in : Müller, dir. 2009, 230-231.

Jacobsthal, P. (1944) : Early Celtic Art, Oxford.

Jenkins, G. K. (1972) : Monnaies grecques, Paris.

Kaenel, G. (1990) : Recherches sur la période de La Tène en Suisse occidentale, Analyse des sépultures, Cahiers d'Archéologie Romande, 50, Lausanne.

Kähler, H. (1948) : Der große Fries von Pergamon: Untersuchungen zur Kunstgeschichte und Geschichte Pergamons, Berlin.

Kruta, V. (2000) : Les Celtes : histoire et dictionnaire, Paris.

Lambot, B., St. Verger et P. Méniel (1995) : Une tombe à char de La Tène ancienne à Semide (Ardennes), Société archéologique champenoise Suppl.

Le Carnyx et la lyre, archéologie musicale en Gaule celtique et romaine (1993) : Catalogue d'exposition, Musées de Besancon, Orléans et Évreux, 1993-1994, Besançon, Musée des Beaux-Arts et d'Archéologie de Besançon.

Le cheval et la danseuse, À la redécouverte du trésor de Neuvyen-Sullias (2007) : Catalogue d'exposition du musée des Beaux-Arts d'Orléans du 13 mars au 26 août 2007 et du musée départemental de Bavay du 15 janvier au 15 juin 2008.

Le Roux, F., et C. Guyonvarc'h (1990) : La civilisation celtique, Rennes.

Lebedynsky, I. (2010): Sarmates et Alains face à Rome - er-ve $^{e}$ siècles, Clermont-Ferrand.

Leclerc G.-L., Comte de Buffon (1756) : Histoire Naturelle, Générale et Particulière avec la description du cabinet du Roi, t. 6, Paris.

Lejars, Th. (2008) : "Les guerriers et l'armement celto-italique de la nécropole de Monte Bibele", in : Vitali \& Verger, dir. 2008, 127-222.

Lubtchansky, N. (2005) : Le cavalier tyrrhénien, représentations équestres dans I'Italie archaïque, BEFAR 320, École française de Rome, Rome.

Maniquet, Chr. (2004) : Le sanctuaire antique des Arènes de Tintignac, Limoges.

— (2005a) : "Découverte d'un formidable dépôt gaulois. Les carnyx de Tintignac", Archéologia, 419, Février 2005, 16 23.

— (2005b) : "The Tintignac celtic warrior hoard", Minerva, vol. $16, n^{\circ} 4$, juillet-août 2005, 29-31.

- (2008) : "Le dépôt cultuel du sanctuaire gaulois de Tintignac à Naves (19)", Gallia 65, 273-326.

— (2009a) : "Le dépôt du sanctuaire de Tintignac à Naves : de nouvelles références pour le site de La Tène", in : Le site de La Tène : bilan des connaissances, état de la question, Archéologie neuchâteloise 43, Actes de la Table ronde internationale de Neuchâtel, 1-3 novembre 2007, 207217.

— (2009b) : Le sanctuaire gaulois de Tintignac (Corrèze), in : Bertrand et al. 2009, 121-148.
— (2009c) : Les guerriers gaulois de Tintignac, Limoges.

Massa-Pairault, Fr.-H. (1985) : Recherches sur l'art et I'artisanat étrusco-italiques à l'époque hellénistique, BEFAR 257, École française de Rome, Rome.

Mathieu, R. (1990) : "Le combat des grues et des pygmées", L'Homme, 30-116, 55-73.

Megaw, J.V.S. (1981) : "Une 'volière' celtique : quelques notes sur l'identification des oiseaux dans l'art celtique ancient", Études offertes à Jean-Jacques Hatt, Revue archéologique de l'Est et du Centre-Est, 32, 3-4, 137-143.

Megaw, R. et Megaw J.V.S. (1989) : Celtic art, from the beginnings to the Book of Kells, Londres (rééd. 2001).

Mille, B. (2007) : "Les trompes gallo-romaines de Neuvyen-Sullias et Saint-Just-sur-Dive, apport d'un étude de laboratoire", in : Le cheval et la danseuse. À la redécouverte du trésor de Neuvy-en-Sullias, 146-155.

Moreau, J., R. Boudet et U. Schaaf (1990) : "Un sanglierenseigne à Soulac-sur-Mer, Gironde", Archäologisches Korrespondenzblatt, t. 20, 439-442.

Moreau, J., D. Ankner, R. Boudet, M. Dhénin, et M. Fecht (1995) : Le sanglier-enseigne gaulois de Soulac-sur-Mer, Musée Archéologique de Soulac-sur-Mer (Gironde).

Moret, P., Cl. Domergue, J. Gomez de Soto, B. Cauuet, G. Nicolini et H. Hautenauve (2001) : "L'or de Tolosa", Catalogue d'exposition présentée au musée SaintRaymond, musée des Antiques de Toulouse du 17 octobre 2001 au 20 janvier 2002, Toulouse.

Müller, F., dir. (2009) : L'art des Celtes, Musée historique de Berne, Landesmuseum Württemberg Stuttgart, Bruxelles.

Pernet, L. (2010) : Armement et auxiliaires gaulois (IIe et er siècles avant notre ère), Protohistoire Européenne 12, Montagnac.

Pernot, M. (1999) : "La métallographie", in : À la recherche du métal perdu - Les nouvelles technologies dans la restauration des métaux archéologiques, Musée archéologique du Val d'Oise, 65-67.

- (2003) : "Étude technique de quelques objets en bronze", in : Rolley, dir. 2003, vol. 1, 265-277, vol. 2, pl. 24.

Perrier, M. (1979) : Le livre du dinandier, Paris.

Peyre, Chr. (1979) : La Cisalpine Gauloise du II au rer siècle avant J.-C., Paris.

Piccardo, P. et M. Pernot (1997) : "Studio analitico stutturale di alcuni vasi celtici in bronzo", La metallurgia italiana, 11, 43-52.

Piggott, S. (1959) : "The carnyx in Early Iron Britain", The Antiquaries Journal, 39, 19-32.

Reinach, A. (1909) : art. "Tuba", in : Dictionnaire des antiquités grecques et romaines, Hachette, Paris, 522-528.

Reinach, S. (1896) : art. "Galea", in : Daremberg, Ch. et Edm. Saglio, Dictionnaire des antiquités grecques et romaines d'après les textes et les monuments, 2-2 (F-G), 1429-1451.

Rolley, Cl., dir. (2003) : La tombe princière de Vix, Paris, 2 vol.

Roncador, R. (2009) : "La "riscoperta" del carnyx di Sanzeno (Val di Non, Trentino, Italia) - Storia degli studi ed inquadramento culturale", in : Grunwald et al. 2009, 547555. 
Rusu, M. (1969) : “Das Keltische Fürstengrab von Ciumesti in Rumanien", Germania, 50, 267-300.

Santrot, J., B. Derion, X. Dupuis et M. Fincker (1996) : "Bronzes et fers de Dax, Landes", Gallia, 53, 289-291.

Sassatelli, G. et E. Govi, éd. (2005) : Culti, forma urbana e artigianato a Marzabotto. Nuove prospettive di Ricerca, atti del convegno di Studi, Bologna juin 2003, Ante Quem, Studi e Scavi, nuova serie 11, Bologne.

Schaaff, Ulr. (1974) : "Keltische Eisenhelme aus vorrömischer Zeit", Festschrift Hundt, Teil 1, Jahrbuch des RömischGermanischen Zentralmuseums Mainz, 21, 149-204.

Schween, J. (2000) : Bemerkungen zum Spiel auf der Nachbildung eines jungbronzezeitlichen Lurenmundstückes vom Typ Brudevælte Nr. 5, in : Hickmann et al., dir. 2000, 307-312.

Stauder, W. (1963) : Les instruments de musique, Paris.

Stead, I.M. et V. Rigby (1999) : The Morel collection, Londres.

Steingräber, St. (1985) : Catalogo ragionato della pittura etrusca, Milan.

Sterckx, Cl. (2009) : Mythologie du Monde Celte, Allemagne.

Szabo, M. (1993) : "Éléments anthropomorphes dans le décor des fourreaux danubiens", in : Briard \& Duval, éd. 1993, 271-286.

Szabo, M. et E.F. Petres (1992) : Decorated Weapons of the La Tène Iron Age in the Carpathian Basin, Inventaria Praehistorica Hungariae 5, Budapest.
Tiefengraber, G., B. Kavur et A. Gaspari, éd. (2009) : Keltske študije II, Studies in Celtic Archaeology. Papers in honour of Mitja Guštin, Protohistoire Européenne 11, Montagnac.

Tirelli, M. (2005) : "Il santuario altinate di Altino-/Altno-", in : Sassatelli \& Govi, éd. 2005, 301-316.

Tissier F. (2007) : Le cyclododécane en archéologie : tests et applications pratiques, Mémoire de Master ConservationRestauration des Biens Culturels, Université de Paris 1.

Vallet, G. et Fr. Villard (1964) : Megara Hyblea, 2. La céramique archaïque, Paris.

Vendries, C. (1993) : "Les Gaulois et la musique au Second âge du Fer", in : Le Carnyx et la lyre, 27-33.

- (1999) : "La trompe, le gaulois et le sanglier, Revue des études anciennes, 101, n³-4, 367-391.

- (2007) : "La trompe de Neuvy. Anatomie d'un objet sonore", in : Le cheval et la danseuse 2007, 120-145.

Verger, St. (1991) : "L'utilisation du répertoire figuratif dans I'art celtique ancien", Histoire de l'Art, 16, 3-17.

Vincent, A. (2007) : "Les musiciens de l'armée romaine impériale", in : Les Dossiers d'Archéologie "Musique à Rome", 320, mars-avril 2007, 34-39.

Vitali, D. (2002) : "L'aristocratie celtique de la Gaule cisalpine. Réflexion pour une première approche", in : Guichard \& Perrin, dir. 2002, 15-28.

Vitali D. et St. Verger, dir. (2008) : Tra mondo celtico e mondo italico. La necropoli di Monte Bibele, Bologne.

Vouga, P. (1923) : La Tène, Monographie de la station publiée au nom de la commission des fouilles de La Tène, Leipzig. 